Portland State University

PDXScholar

Fall 12-8-2014

\title{
Chemical and Petrographic Survey of Large, Igneous- Textured Inclusions in Ordinary Chondrites
}

Katherine Armstrong

Portland State University

Follow this and additional works at: https://pdxscholar.library.pdx.edu/open_access_etds

Part of the Geology Commons, and the The Sun and the Solar System Commons Let us know how access to this document benefits you.

Recommended Citation

Armstrong, Katherine, "Chemical and Petrographic Survey of Large, Igneous-Textured Inclusions in Ordinary Chondrites" (2014). Dissertations and Theses. Paper 2070.

https://doi.org/10.15760/etd.2069

This Thesis is brought to you for free and open access. It has been accepted for inclusion in Dissertations and Theses by an authorized administrator of PDXScholar. Please contact us if we can make this document more accessible: pdxscholar@pdx.edu. 
Chemical and Petrographic Survey of Large, Igneous-Textured Inclusions in Ordinary Chondrites

by

Katherine Armstrong

A thesis submitted in partial fulfillment of the requirements for the degree of

Master of Science

in Geology

Thesis Committee:

Alex M. Ruzicka, Chair

Richard C. Hugo

R. Benjamin Perkins

Portland State University

2014 


\begin{abstract}
Our inventory of material from the early solar system includes large, igneoustextured inclusions in $\mathrm{O}$ chondrites, whose origin and relationship to their host meteorite is unclear. These inclusions occur in approximately $4 \%$ of $\mathrm{O}$ chondrites, and are mineralogically, petrographically, and chemically diverse. Petrographic and chemical data from 29 inclusions from 23 host meteorites were collected with optical light and scanning electron microscopy, allowing for the determination of major phase modal abundance and major element bulk chemistry. No correlation between any inclusion property and host meteorite type were found, but some trends were observed. Nine of the inclusions show strong evidence, such as radial variations in texture and chemistry, for having crystallized as a free-floating droplet in a space environment, and may share the same formation process as chondrules. One inclusion is almost certainly shockmelted material that intruded into the host material. Thirteen inclusions have bulk chemistry patterns that suggest the material was vapor fractionated; the remaining sixteen are essentially chondritic, i.e., unfractionated. Broadly, the data support the conclusions of Ruzicka et al. (1998, 2000), which divided large inclusions into Na-poor (vapor fractionated) and Na-rich (unfractionated) groups, suggesting at least two different origins. There is no evidence that any of the inclusions studied formed by igneous differentiation.
\end{abstract}




\section{Contents}

$\begin{array}{ll}\text { Abstract } & \text { i }\end{array}$

List of Tables $\quad$ iv

List of Figures $\quad$ V

1 Introduction 1

1.1 Scope of this study . . . . . . . . . . . . . . . . . . . . 1

1.2 Meteorites and their record of the early solar system . . . . . . . . 1

1.3 Early solar system processes recorded by chondrites . . . . . . . . . . 2

1.4 Large Inclusions . . . . . . . . . . . . . . . . . . . . 8

1.5 Proposed formation models . . . . . . . . . . . . . . . . . 12

1.6 Goals of this study and future work . . . . . . . . . . . . . . 17

2 Methods and Samples $\quad 19$

2.1 Samples and Data Collection . . . . . . . . . . . . . . . . . . 19

2.2 Data Analysis . . . . . . . . . . . . . . . . . 23

$\begin{array}{lll}3 & \text { Results } & 27\end{array}$

4 Discussion $\quad 44$

4.1 Drop-formed inclusions . . . . . . . . . . . . . 45

4.2 Shock melt . . . . . . . . . . . . . . . . . . 50

4.3 Trends in bulk chemistry . . . . . . . . . . . . . . . . . . . 52

4.4 Olivine "donuts" . . . . . . . . . . . . . . . . . . . . 63

4.5 Nearby metal nodules . . . . . . . . . . . . . . . . 65

4.6 Brecciation .......................... 68

$\begin{array}{lll}5 & \text { Summary and Conclusions } & 70\end{array}$

$\begin{array}{ll}\text { References Cited } & 72\end{array}$

$\begin{array}{ll}\text { Appendix } & 79\end{array}$

A Chemical and petrographic descriptions of individual inclusions $\quad 79$

A.1 NWA 8231 (CML 0368) H4-6 . . . . . . . . . . . . . . . . 79

A.2 Tamdakht (CML 0646) H5 . . . . . . . . . . . . . . . . . . 82

A.3 NWA 7873 (CML 0615) H5-6 . . . . . . . . . . . . . 87 
A.4 Etter (AMNH 4462) H6 . . . . . . . . . . . . . . . . . . 89

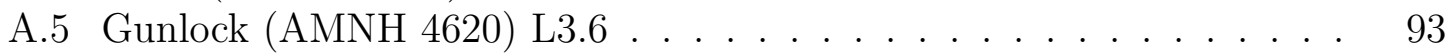

A.6 Khohar $($ AMNH 4245) L3.6 . . . . . . . . . . . . . . . . . . 95

A.7 NWA 7869 (CML 0143) L3.7 . . . . . . . . . . . . . . . . . . 98

A.8 NWA 7872 (CML 0359) L3.7 . . . . . . . . . . . . . . . . 105

A.9 NWA 869 (CML 0074) L3-6 . . . . . . . . . . . . . . . . . 108

A.10 NWA 8141 (CML 0014) L3-6 . . . . . . . . . . . . . . . . 111

A.11 Cynthiana (AMNH 0369) L4 . . . . . . . . . . . . . . . . 114

A.12 Kramer Creek (AMNH 4428) L4 . . . . . . . . . . . . . . . 117

A.13 McKinney (AMNH 0391) L4 . . . . . . . . . . . . . . . . . . . . . 119

A.14 NWA 7870 (CML 0173) L4 . . . . . . . . . . . . . . . . 125

A.15 NWA 7871 (CML 0178) L6 . . . . . . . . . . . . . . . . 128

A.16 Palo Verde Mine (CML 0185) L6 . . . . . . . . . . . . . . . 131

A.17 Ragland (AMNH 4825) LL3.4 . . . . . . . . . . . . . . . . 134

A.18 Parnallee (CML 0524) LL3.6 . . . . . . . . . . . . . . . . . . 137

A.19 Lut 005 (CML 0740) LL3.7 . . . . . . . . . . . . . . . . 143

A.20 Richfield (AMNH 4887) LL3.7 . . . . . . . . . . . . . . . 146

A.21 NWA 4859 (CML 0272) LL5 . . . . . . . . . . . . . . . . 149

A.22 NWA 8232 (CML 0372) LL5 . . . . . . . . . . . . . . . 155

A.23 Oberlin (AMNH 3832) LL5 . . . . . . . . . . . . . . . 158 


\section{List of Tables}

2.1 List of samples . . . . . . . . . . . . . . . . . . . 20

3.1 Basic properties of the inclusions $\ldots \ldots \ldots \ldots \ldots \ldots \ldots \ldots$

3.2 Physical properties of the inclusions . . . . . . . . . . . . . . . . . . . $\quad 29$

3.3 Modal abundance of all inclusions . . . . . . . . . . . . . . . 31

3.3 Bulk chemsitry of all inclusions . . . . . . . . . . . . . . 34

3.4 Average compositions of olivine and low-Ca pyroxene . . . . . . 38

3.5 Average composition of clinopyroxene . . . . . . . . . . . 40

3.6 Average composition of feldspar . . . . . . . . . . . . . . . 41 


\section{List of Figures}

1.1 Example of chondrules . . . . . . . . . . . . . . . . 3

1.2 Example of increasing metamorphic grade . . . . . . . . . 5

1.3 Elemental abundances of the chondrite groups . . . . . . . . . . 6

1.4 Examples of large igneous inclusions . . . . . . . . . . . . 9

1.5 CMAS plot of data from Ruzicka et al. (2000). . . . . . . . . . . . 13

1.6 Bulk composition of inclusions in the Buzzard Coulee meteorite, from Ruzicka et al. (2012a) . . . . . . . . . . . . . . . . . . . . . 16

1.7 Ol-Pl-Qz plot of inclusion compositions from Ruzicka et al. (2012a) . 18

2.1 Example optical image . . . . . . . . . . . . . . . . . 22

2.2 Example false-color phase map . . . . . . . . . . . . . . . 25

2.3 Illustration of method used to obtain modal abundance . . . . . . . 26

3.1 Modal abundance of all inclusions . . . . . . . . . . . . . . . 31

3.2 Average olivine and pyroxene phase compositions . . . . . . . . . 37

3.3 Average feldspar phase compositions . . . . . . . . . . . . . 38

3.4 Normative olivine-plagioclase-quartz ternary . . . . . . . . . . . . 43

4.1 Examples of droplet-formed objects . . . . . . . . . . . . . . . 48

4.2 Bulk chemistry of drop-formed inclusions . . . . . . . . . . . . 49

4.3 Example of likely shock melt . . . . . . . . . . . . . . . . 50

4.4 Potassium-enriched inclusions . . . . . . . . . . . . . . . 51

$4.5 \mathrm{Mg} / \mathrm{Al}$ plotted against $\mathrm{Na} / \mathrm{Al} \ldots \ldots \ldots \ldots . \ldots . \ldots . \ldots 53$

$4.6 \mathrm{Al} / \mathrm{Mg}$ plotted against $\mathrm{Na} / \mathrm{Mg} \ldots \ldots \ldots \ldots . \ldots . \ldots . \ldots 54$

4.7 Bulk chemistry of inclusions in a high-type host . . . . . . . . 56

4.8 Bulk chemsitry of unfractionated inclusions in a low-type host . . . 57

4.9 Bulk chemistry of feldspar-enriched inclusions . . . . . . . . . . 59

4.10 Bulk chemistry of volatile-depleted inclusions . . . . . . . . . . . . 61

4.11 Bulk chemistry of refractory-enriched inclusions . . . . . . . . . 62

4.12 Olivine "donuts" . . . . . . . . . . . . . . . . . . . . . . . 64

$4.137873-\mathrm{I} 1$ and adjacent metal/sulfide nodule $\ldots \ldots \ldots \ldots 67$

4.14 Brecciated region of Glk-I1 . . . . . . . . . . . . . . . . . 68

4.15 Offset phenocryst within $7873-\mathrm{I} 1 \ldots \ldots \ldots$ 


\section{Introduction}

\subsection{Scope of this study}

Studies of meteorites and their constituents have greatly increased our knowledge of the formation and dynamics of the early solar system, as they carry a record of that time that has been obliterated on the Earth and other large solar system bodies. The chondrites, in particular, have allowed us to place many constraints on the processes that led to planetary formation.

A small fraction of $\mathrm{O}$ chondrites contain large, igneous-textured inclusions, the origins of which are poorly understood. This work details the petrology and major element bulk chemistry of 29 of these inclusions from a diverse array of host meteorites. It is the first step in a large survey of these inclusions, the ultimate goal of which is to constrain their history and relationships to each other and their host meteorites in order to further illuminate the details of the early solar system and planetary formation.

\subsection{Meteorites and their record of the early solar system}

\subsubsection{Meteorites}

Meteorites are fragments of extraterrestrial objects in the solar system, delivered to Earth after having been ejected from their parent body and sent into an Earthcrossing orbit. They can be stones, iron-nickel metal alloys, or a mixture of both;

the stony-irons (e.g., Weisberg et al., 2006). Their varying components and chemical compositions are believed to reflect early solar system processes, including the initial accretion of dust-sized particles into planetesimals and processes undergone by their 
parent bodies such as differentiation.

Chondrites A primary chemical distinction can be used to broadly categorize stony meteorites into chondrites and achondrites. Achondrites are believed to originate from parent bodies that, like the Earth, underwent differentiation: global melting that allowed for the physical and chemical separation of material to form a core-mantle structure (Hutchison, 2004). Chondrites, conversely, have elemental abundances that are, except for the most volatile elements, which tend to remain gaseous, almost identical to that of the solar photosphere (McSween, 1987). Because of this, they are generally believed to comprise primitive material that has remained largely unchanged since the formation of the solar system (Hutchison, 2004).

One class of chondrites, the ordinary or $\mathrm{O}$ chondrites make up a large majority of known meteorites, and thus are our most abundant examples of primitive solar system material. The O chondrites comprise three groups, H, L, and LL (e.g., Wood, 2005), which have distinct chemistry and are believed to represent three unique parent bodies (Burbine et al., 2002). All samples in this study are O chondrites.

\subsection{Early solar system processes recorded by chondrites}

Although the chondrites do not appear to have originated on a parent body that experienced global melting, they nonetheless are believed to originate from asteroidal parent bodies (Burbine et al., 2002) that would have experienced some post-accretional processing. Some of these secondary processes, including chondrule formation, thermal metamorphism, volatile loss, and shock, are relevant for the interpretation of the samples in this study and so are briefly reviewed here. 


\subsubsection{Chondrules}

Chondrites are named for chondrules, which are a major and conspicuous constituent of most groups of chondritic meteorites (figure 1.1). Chondrules are typically defined as more-or-less spherical, $500 \mu$ m-diameter assemblages of silicate minerals that experienced melting and crystallization as free-floating droplets in space (Friedrich et al., 2014). Because chondrules are such a dominant component of chondrites, their formation is ar-

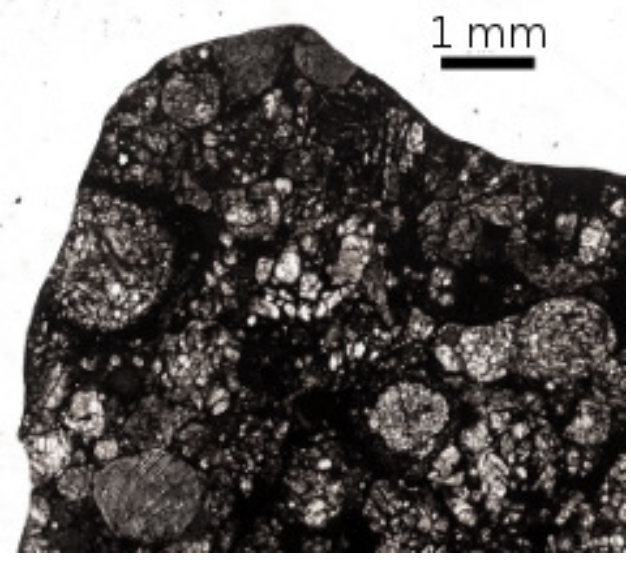

Figure 1.1: Chondrules are spherical assemblages of crystalline silicates, often in a glassy, opaque matrix, as seen here in NWA 7869. (Viewed with plane transmitted light.) guably the most significant process recorded by chondrites. Despite decades of study, this process remains poorly understood and extremely controversial (e.g., Lauretta et al., 2006; Wood, 1996).

They are usually dominated by ferromagnesian silicates, often with a feldspathic mesostasis (e.g., Hewins, 1997), however, chondrules are quite diverse, both texturally and mineralogically (Jones et al., 2005). They have thus been subdivided into eight types, based on their texture and mineralogy, described by acronyms such as BO (barred olivine), RP (radial pyroxene) and POP (porphyritic olivine pyroxene) (Gooding and Keil, 1981). Hewins (1997) provides a detailed review of the proposed mechanisms for chondrule formation. 
1 Introduction

\subsubsection{Thermal Metamorphism}

Although the chondrite parent bodies did not undergo differentiation, most $\mathrm{O}$ chondrites are metamorphosed to various degrees, which suggests that those bodies were thermally processed. Van Schmus and Wood (1967) originally proposed the scheme on which modern classification is based. Those authors divided chondrites into six petrologic types depending on the degree of thermal and aqueous alteration. Types 1 and 2 were suggested to be unmetamorphosed samples that had experienced aqueous alteration, while types 3-6 were defined as increasing levels of thermal metamorphism. The original thermal gradation scheme has been refined and revised since 1967, primarily with the addition of subgrades (called "subtypes") 3.0-3.9 to better describe those samples with minor processing by Sears et al. (1980) and Sears and Dodd (1988) The degree of thermal processing is low for subtypes 3.0-3.3, and progressively higher from 3.4-3.9 (Sears and Dodd, 1988; Huss et al., 2006).

The textural, chemical, and mineralogical products of thermal metamorphism in O chondrites are well established. As chondritic material is heated, the constituent phases change to reflect the new thermodynamic conditions: new phases form, grains grow, and phases change their structure and/or chemistry. The physical effects of thermal metamorphism include matrix recrystallization, chondrule glass devitrification, equilibration of olivine and other silicates, bulk volatile loss, and the nucleation of new phases (Huss et al., 2006).

Texturally, chondrules become less distinct with increasing metamorphic grade, becoming progressively indistinguishable from matrix at types 5-6 (figure 1.2). The matrix, which is opaque and fine-grained or glassy for type 3, recrystallizes into coarser 
grains and becomes translucent and then transparent. In addition, small grains of metal and sulfide mobilize and coalesce into larger, amorphous blebs (e.g., Huss et al., 2006 and references therein).
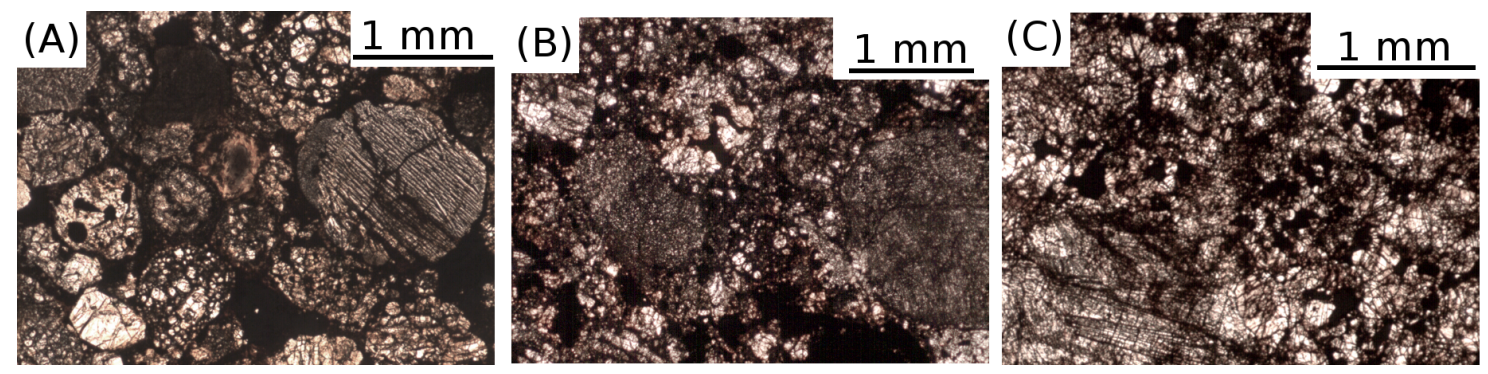

Figure 1.2: Examples of petrologic types $3(\mathrm{~A}), 4(\mathrm{~B})$, and $6(\mathrm{C})$, viewed in plane transmitted light. With increasing metamorphic grade, chondrules become less distinct, opaque matrix recrystallizes, and new phases nucleate and grow.

\subsubsection{Volatile Loss}

The CI group of carbonaceous chondrites has the closest compositional correlation with the solar photosphere, and hence are widely believed to be the best representation of bulk solar system composition, excepting of course the lightest and most volatile elements (hydrogen, carbon, nitrogen, oxygen) and the noble gases (Anders and Grevesse, 1989). Comparatively, all other known rocky solar system objectsthe terrestrial planets, and other meteorite classes- are depleted in volatile elements (Davis, 2006). The different meteorite groups have differing depletions, but for many there is an overall smooth trend as a function of volatility: the more volatile the element, the more strongly it is depleted (see figure 1.3). 


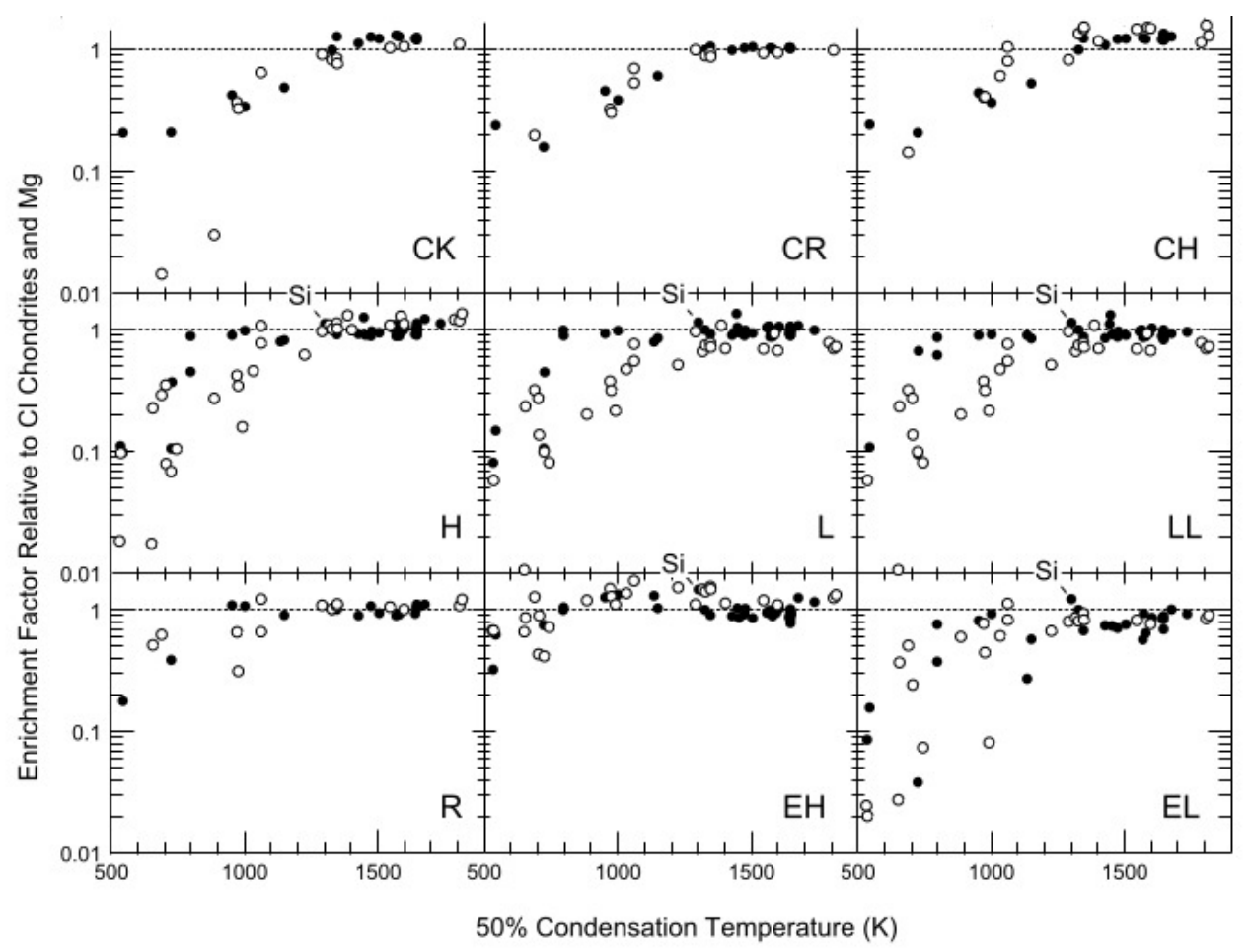

Figure 1.3: All chondrite groups, relative to CI chondrites, are depleted in the most volatile elements, shown here in a figure from Davis (2006) where elements are arranged in order of decreasing volatility, as measured by $50 \%$ condensation temperature, or $\mathrm{T}_{\mathrm{c}, 50}$. Solid data points are lithophile elements, clear data points are siderophile/chalcophile.

Elements are classified as refractory if they have a $50 \%$ condensation temperature $\left(\mathrm{T}_{\mathrm{c}, 50}\right)$ greater than that of the major rock-forming elements $\mathrm{Mg}, \mathrm{Si}$, and $\mathrm{Fe}(\mathrm{T}>$ $1335 \mathrm{~K}$ at solar composition and $\mathrm{P}=10^{-4}$ atm), moderately volatile if their $\mathrm{T}_{\mathrm{c}, 50}$ is less than the refractory elements, but greater than FeS $(1335>\mathrm{T}>665 \mathrm{~K})$, and highly volatile if their $\mathrm{T}_{\mathrm{c}, 50}$ is less than that of FeS (665 K) (Lodders, 2003).

Trends in depletions that are dependent on volatility can be explained by either evaporation or condensation. It is difficult to ascertain which of these kinetic processes was at work, especially considering that further processing, such as metasomatism, 
could have altered the effects of the original process. Typically, it is the comparison of elements with certain geochemical properties that is used to illuminate the processes that were likely at work. For instance, the elements in $\mathrm{O}$ chondrites follow different trends depending on whether they are siderophile or lithophile, indicating that a metal-silicate fractionation process occurred and volatility fractionation occurred under different conditions for the metals than for the silicates (Davis, 2006).

\subsubsection{Shock}

Collisions have played an influential role in the evolution of solar system objects. A hypervelocity impact creates a shock wave that essentially instantaneously creates temperatures of thousands of degrees $\mathrm{C}$ and pressures of tens of $\mathrm{GPa}$ as it propagates through the target (e.g., Melosh, 1989). Depending on the size of the impact, large volumes of rock can be vaporized or melted.

At the micron scale, shock wave propagation through a heterogeneous material is a complex and chaotic process. Peak temperatures and pressure can vary by an order of magnitude between grains or within single grains due to interactions between shock waves that are reflected or refracted at grain interfaces and cracks or voids (Sharp and DeCarli, 2006).

These extreme conditions can profoundly alter rocks near an impact site. Effects on constituent minerals of the target rock include deformation, such as fracturing or mosaicism (deformation of the crystal lattice), and transformation, either via solidstate transformation to a high $\mathrm{P} / \mathrm{T}$ polymorph or melting and recrystallization (e.g., Stöffler et al., 1991). Evidence of shock in the form of high $\mathrm{P} / \mathrm{T}$ phases, deformed minerals, and melt veins and pockets is commonly observed in O chondrites (Dodd 
and Jarosewich, 1979).

\subsection{Large Inclusions}

Uniquely to O-chondrites, some samples contain "large igneous-textured inclusions" (Ruzicka et al., 1998, 2000). Individual inclusions have been recognized and studied, but relatively few survey-type studies have been conducted on igneous inclusions as a group. In 1997, Bridges and Hutichison surveyed the meteorite collection of the British Museum of Natural History to determine how abundant these inclusions are, and found that they occur in approximately $4 \%$ of $\mathrm{O}$ chondrites.

Figure 1.4 shows several such objects in thin section and hand sample. These inclusions typically have a diameter $5 \mathrm{~mm}$ (Ruzicka et al., 1995, 1998; Bridges and Hutchison, 1997) but have been observed to be as large as $5 \mathrm{~cm}$ (Binns, 1967; Prinz et al., 1988). They are much larger (diameters of about an order of magnitude larger) than most chondrules in $\mathrm{O}$ chondrites, which are by far more numerous within the same host meteorite (Hutchison, 2004). These inclusions are almost always highly depleted in metal and sulfide relative to their host meteorite (Ruzicka et al., 1998, 2000). Though they are all igneous, they exhibit a large range of textures, mineralogies, and bulk compositions, and may have rounded or irregular edges, suggesting a variety of formation processes. 


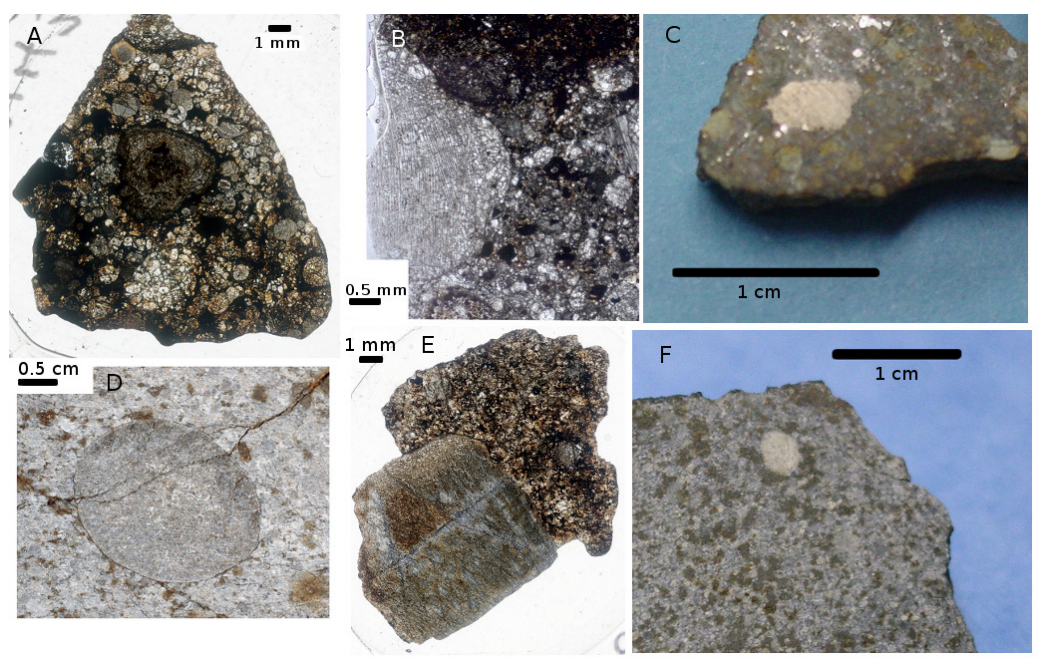

Figure 1.4: Examples of large igneous inclusions in thin section (A, NWA 7869; B, NWA 7872; and E, NWA 7871) and hand sample (C, Parnallee; D, NWA 4859; and F, Tamdakht).

Ruzicka et al. (1998; 2000) described a diverse set of igneous inclusions that together show evidence for a dynamic early solar system. Among the set are inclusions that appear to have crystallized as discrete, free-floating droplets in space, as well as others that appear to be fragments of larger igneous objects. Chemically, the inclusions in this study (Ruzicka et al., 1998, 2000) were classified as either Narich, with essentially chondritic compositions, or Na-poor, with depletions in volatile elements. There are inclusions that show evidence for metamorphism and metasomatism (chemical alteration by hydrothermal and other fluids) both before and after they were fragmented into clasts, and before and after their incorporation into the host chondrite. The diversity of these objects is reflected in the numerous formation models have been proposed in the literature, which are in some cases quite complex (Ruzicka et al., 1995, 1998, 1999, 2012a; Dodd and Jarosewich, 1976; Fodor and Keil, 1976; Jamsja and Ruzicka, 2010; Binns, 1967; Weisberg et al., 1988; Hutchison et al., 
1988).

\subsubsection{Possible link to differentiated meteorites}

Ruzicka et al. (2012b; 2012a) found evidence that igneous clasts in the Buzzard Coulee chondrite bear a striking chemical similarity to silicates in type IIE iron and type IVA stony iron meteorites. This suggests that there may be some link between certain inclusions and differentiated meteorites, or perhaps even a link between chondrites and differentiated meteorites.

Two clasts that share a similar lithology (Bz-1 and Bz-2) have a composition that would be expected for a cumulate that formed on the pyroxene-silica cotectic, and a texture that is consistent with concurrent crystallization of pyroxene and cristobalite (Ruzicka et al., 2012a). Previous studies of the Steinbach IVA stony iron meteorite suggested that the silicates of Steinbach had also originated by fractional crystallization along the pyroxene-silica cotectic, though Steinbach is coarser and tridymite was present instead of cristobalite (Ruzicka and Hutson, 2006).

The third Buzzard Coulee clast that was studied (Bz-3) was chemically unlike any other large igneous inclusion found in an O-chondrite, but is instead similar in composition to silicates in the Weekeroo Station and Miles IIE silicate-bearing iron meteorites (Ruzicka et al., 1999, 2012a; Ruzicka and Hutson, 2010). Previous studies of these materials suggested an origin by partial melting during differentiation, and the same model appears to hold for Bz-3. 


\subsubsection{Possible link to chondrules}

Due to their diversity, the term "chondrule" is somewhat ambiguously defined, and it can be reasonably applied to any discrete object in a chondrite that has an igneous (i.e., crystallized from a melt) texture (Binns, 1967). For the purposes of this work, a distinction is made between "normal" chondrules and what are here termed, generically, "igneous-textured inclusions." A subset of large igneous inclusions, the "megachondrules," appear to be analogous to chondrules (Ruzicka et al., 1998; Bridges and Hutchison, 1997; Weyrauch and Bischoff, 2012), with similar formation processes and histories, and so may help constrain our understanding of chondrule formation.

These "megachondrules" show evidence, such as a generally spherical shape and a radial variation in texture, of having crystallized as free-floating droplets (Ruzicka et al., 2000). This has led to the suggestion that they share an origin and history with chondrules, simply on a larger scale (Bridges and Hutchison, 1997; Weyrauch and Bischoff, 2012). Their large sizes make them more amenable to study with a variety of techniques. For example, their bulk compositions can be more reliably defined using SEM mapping techniques, and their bulk chemical and isotopic compositions can be better studied by obtaining separates. In addition, the large sizes make the inclusions less susceptible to open-system exchange (Ruzicka et al., 1998), meaning that their compositions should be more pristine. If they are simply large chondrules, constraining the history of the melt could provide valuable insight to the contentious subject of chondrule formation. 


\subsection{Proposed formation models}

Large igneous inclusions in O-chondrites were all crystallized from melt volumes on the order of 1-100 $\mathrm{cm}^{3}$, the origins of which are poorly understood. To date, models proposed for their formation include (1) shock melting of ordinary chondrites with an associated loss of metal and sulfide (e.g., Dodd and Jarosewich, 1976; Fodor and Keil, 1976; Ruzicka et al., 1998, 2000; Jamsja and Ruzicka, 2010); (2) melting of vaporfractionated condensate mixtures (Ruzicka et al., 1998, 2000); (3) chondrule formation involving a larger melt production volume than typical for chondrules (Ruzicka et al., 1998, 2000; Binns, 1967; Weisberg et al., 1988); and (4) igneous differentiation occurring within planetesimals sampled by ordinary chondrite parent bodies (Hutchison et al., 1988; Ruzicka et al., 1995, 2012a). These models are described in more detail below.

\subsubsection{Shock Melting}

Several workers have explained certain large igneous inclusions as having crystallized from shock-melted material. Binns (1967), Dodd and Jarosewich (1976), Fodor and Kiel (1976), and Rubin (1981), among others, each interpret different igneous-textured lithic clasts in chondrites as chondritic material that has been shock melted and lost its metal and sulfide, either through vapor fractionation or liquid immiscibility.

As described above, impacts can create melt in large volumes at the impact site, but also as discrete pockets contained in otherwise unmelted rock. Ruzicka et al. (1998; 2000) hypothesize that Na-rich inclusions (both drop-formed and clasts) with chondritic or slightly higher $\mathrm{Na} / \mathrm{Al}$ values and chondritic abundances of volatile ele- 
ments could be shock melts. The chondritic compositions of these inclusions overlap that of melt-pocket glasses (see figure 1.5), though the inclusions are much larger and crystalline as opposed to glassy, reflecting a slower cooling rate. This could possibly indicate that while the melt pockets were constrained to pore space, the larger inclusions were not.

Some of the Na-rich inclusions have chondritic abundances of a feldspathic component, which Ruzicka et al. (1998) take as evidence that the precursor melt was probably a total melt of a chondrite source. Other inclusions have a superchondritic abundance of feldspar. Given that feldspar melts before olivine and pyroxene, the authors infer that the precursor melt was an incipient or partial melt of a chondritic precursor.

Differences in the overall shape and texture of shock melted material could be explained if the drop-formed inclusions formed through splashing or jetting (Ki-

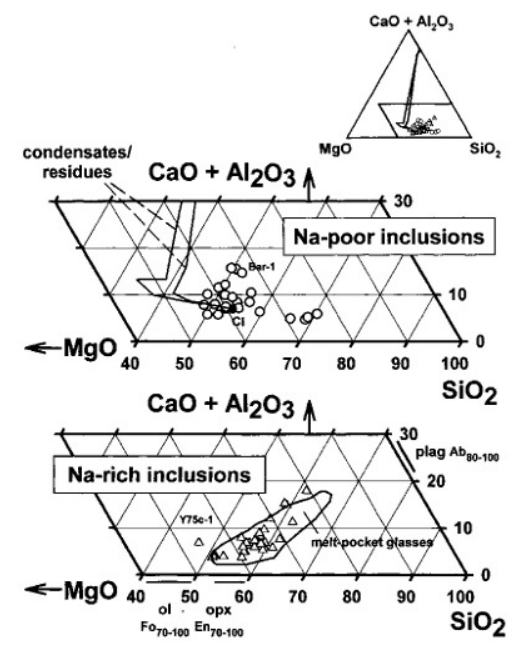

Figure 1.5: Data points for several inclusions plotted on a $\left(\mathrm{CaO}+\mathrm{Al}_{2} \mathrm{O}_{3}\right)-\mathrm{MgO}-\mathrm{SiO}_{2}$ (CMAS) ternary diagram, compared to meltpocket glasses produced by localized shock melting in O-chondrites and to calculated compositions of equilibrium condensates or residues in a chondritic system. Although the compositions overlap, Na-rich and Na-poor inclusions form differing clusters and trends. Figure from Ruzicka et al. (2000). effer, 1975) of molten material that then crystallized rapidly in a space environment, while the irregular clasts are fragments of larger, slower-cooling melt veins or sheets (Ruzicka et al., 1998). 


\subsubsection{Vapor-fractionated condensates}

Sodium-poor inclusions were inferred by Ruzicka et al. (1998) to have had a complex history, including a high-temperature formation of a precursor melt that included vapor fractionation, reequilibration at a lower temp, remelting, brecciation, and finally a metasomatic (fluid-driven) influx of volatile elements. The first equilibration is distinct from the second, as the rims of some objects are enriched in volatiles compared to the cores.

Na-poor inclusions with relatively low $\mathrm{Na} / \mathrm{Al}$ values and subchondritic abundances of volatile elements could have formed by the melting of condensate mixtures. Two different trends, trend $\mathrm{A}$ and trend $\mathrm{B}$ were identified. Trend $\mathrm{A}$ was interpreted to represent condensate mixtures enriched in a refractory, olivine-rich component; and Trend B was interpreted to represent condensate mixtures depleted in a refractory, olivine-rich component (Ruzicka et al., 1998, 2000). Trend A precursors were inferred to be pre-existing mixtures of vapor-fractionated components, including a high-temperature refractory component, and a low-temperature CI-like component.

Trend B inclusions vary widely in $\mathrm{Mg} / \mathrm{Si}$ ratios. The most olivine-rich inclusions have compositions that would be expected for the first condensates or late residues of a vapor-fractionated system. Similarly, the silica-rich inclusions could be explained as condensates from a silica-rich gas resulting from fractional vaporization, or as the remaining condensates after olivine-rich condensates were removed. 


\subsubsection{Megachondrules}

Ruzicka et al. (1998) subdivided large inclusions into two textural classes, lithic clasts and droplet-formed "megachondrules." Megachondrules are spherical (or fragments of objects that were once spherical) and appear to have crystallized as free-floating droplets, as they display radial variations in texture and chemistry that suggest an successive crystal growth and an interaction with their surroundings. Hence, it was suggested (Ruzicka et al., 1998, 2000; Weisberg et al., 1988; Prinz et al., 1988), that megachondrules formed via the same (poorly understood and controversial) mechanism as "normal" chondrules.

Megachondrules most frequently ( $50 \%$, Weyrauch and Bischoff, 2012) exhibit a barred olivine or barred olivine pyroxene $(\mathrm{BO} / \mathrm{BOP})$ texture that, besides being larger, is otherwise similar to normal $\mathrm{BO} / \mathrm{BOP}$ chondrules. The other textures observed- cryptocrystalline, radial pyroxene, microporphyry- are also common in normal chondrules. This is consistent with the findings of Weyrauch and Bischoff (2012), who examined megachondrules from 64 chondrites. It is also notable, because barred olivine is one of the rarer textures of "normal" chondrules, accounting for only $2 \%$.

Like previous surveys (Bridges and Hutchison, 1997; Ruzicka et al., 1998, 2000), Weyrauch and Bischoff found no correlation to megachondrule type or abundance based on petrologic type, nor any correlation between megachondrule type and host chondrite group. 


\subsubsection{Igneous Differentiation}

At least a few large igneous inclusions (Hutchison et al., 1988; Ruzicka et al., 2012a, 1995) with an achondritic bulk composition have been suggested to have derived from precursor melts that formed via igneous differentiation.

Hutchison et al. (1988) discuss a clast in Barwell (L6) that appears to be an olivine plagioclase cumulate, as the plagioclase crystals are too large to be an interstitial phase, and texture is primary. They infer that the clast formed by fractional crystallization of a melt with $\mathrm{H}-$ group O signature.

The Buzzard Coulee chondrite has at least three inclusions that have textures and phase compositions best explained by igneous crystallization (Ruzicka et al., 2012a). These inclusions show no evidence for a volatility-controlled fractionation (see figure 1.6), nor are their compositions consistent with a shock-derived melt.

Compositionally, two inclusions are near the pyroxene-silica cotectic, and a

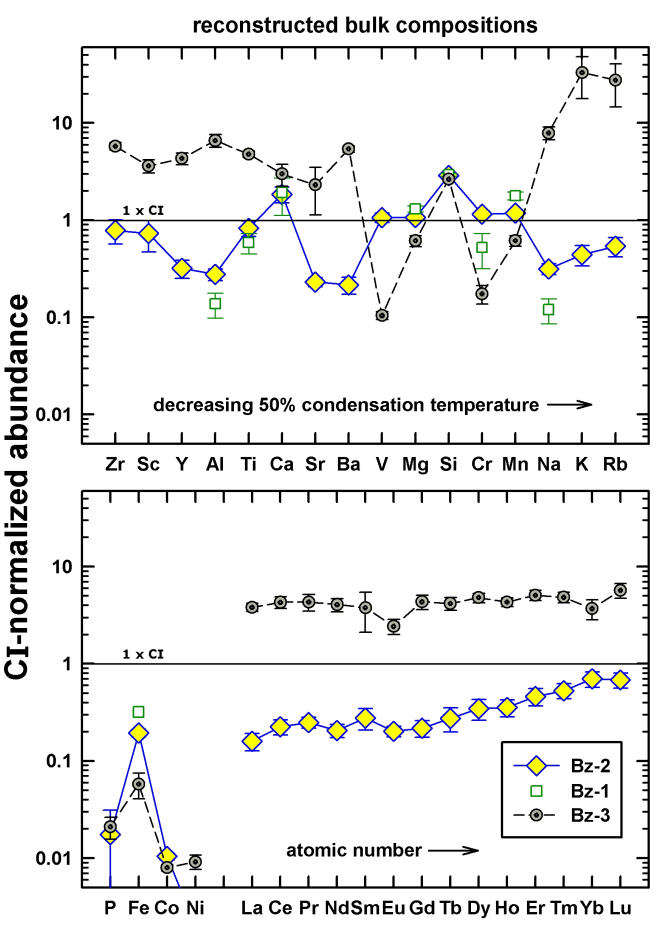

Figure 1.6: shows abundances in the $\mathrm{Bz}$ inclusions, with elements arranged in the top panel by volatility ( $50 \%$ condensation temperatures). The patterns are irregular, hence there is no evidence that the composition of these inclusions was controlled by vaporfractionation processes, which would be be the case if they had formed from the melting of condensate mixtures (Ruzicka et al., 2012a,b) 
third lies close to the olivine-pyroxene reaction boundary, consistent with having formed via igneous differentiation from a partial melt of an O-chondrite-like precursor (see figure 1.7).

\subsection{Goals of this study and future work}

As noted above, the relationship of large igneous inclusions to other early solar system material is unclear, and so constraints on their origins and history have the potential to enhance our understanding of solar system evolution. This study is the first step of a detailed study and survey of a large sampling of these inclusions. Here, the petrography and major element bulk chemistry of 28 inclusions is described. These samples come from all three types of $\mathrm{O}$ chondrites, and various levels of metamorphic grade.

These results are compared to what has been done before, and used to infer potential origins of the inclusions and processing they may have undergone. These

data will allow for further study, particularly trace element (SIMS), dating (Hf-W and $\mathrm{I}-\mathrm{Xe}$ ), and $\mathrm{O}$ isotope that will ultimately yield insight into the formation of these inclusions. 


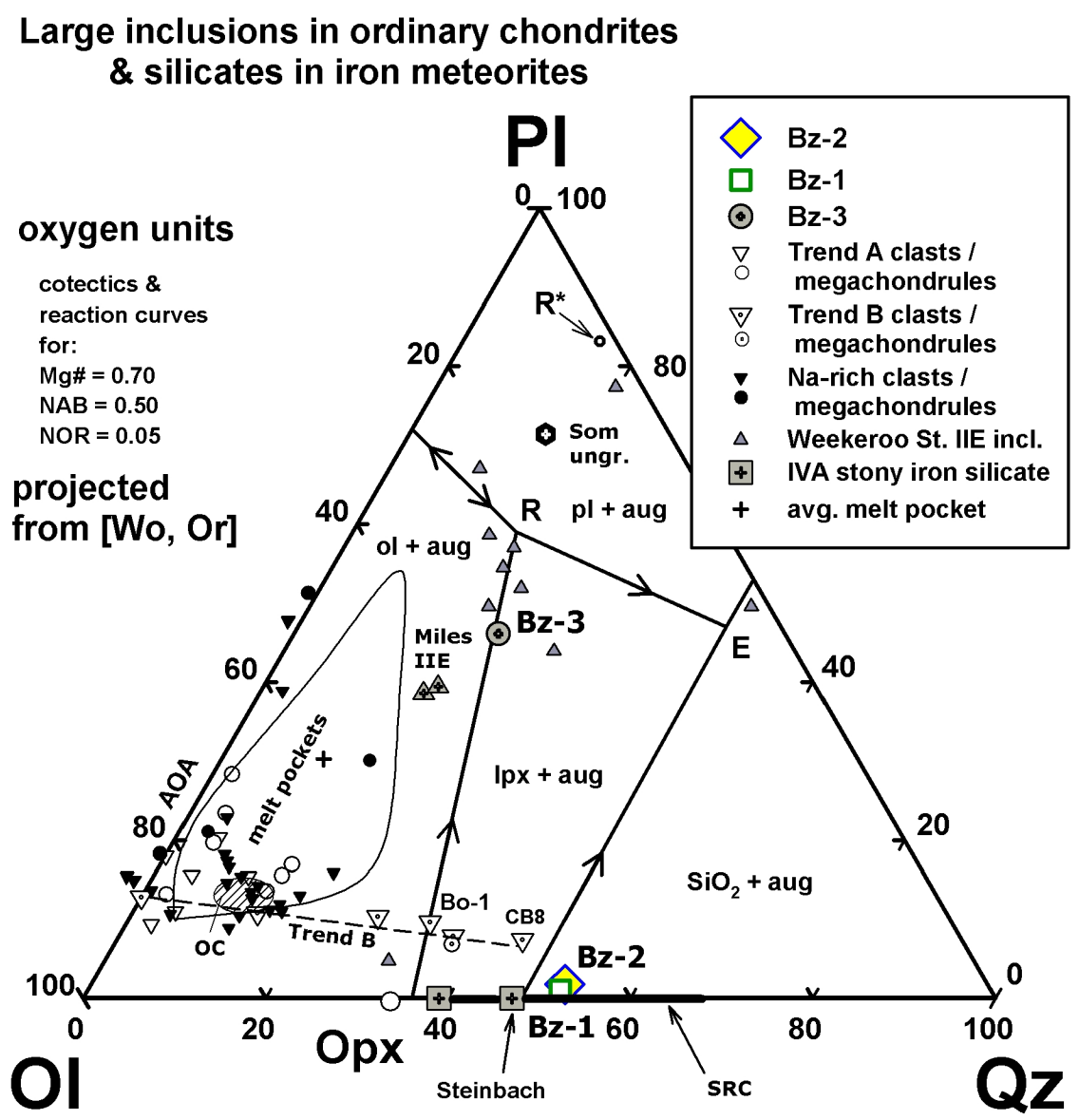

Figure 1.7: Plot of inclusion compositions from Ruzicka et al. (2012a) 
2 Methods and Samples

\section{Methods and Samples}

\subsection{Samples and Data Collection}

For this study, petrographic analyses of 29 inclusions were conducted with optical light microscopy (OLM) and scanning electron microscopy (SEM), and their major phase chemistry was investigated with SEM energy dispersive spectroscopy (EDS). Some of these data were then verified with electron microprobe analyses (EMPA).

Any object in an $\mathrm{O}$ chondrite that has an igneous texture and a cross-sectional diameter atypically large as compared to chondrules in the host was considered for this survey. O chondrite samples owned by Cascadia Meteorite Laboratory (CML) were examined for inclusions, and 17 inclusions in 16 polished thin sections from 14 meteorites were identified (see table 2.1). An additional 12 inclusions studied are in 10 meteorites loaned from the American Museum of Natural History.

Nine of the CML-owned meteorites containing inclusions were unclassified O chondrites, requiring classification before analyses of the inclusions could begin. Petrographic and chemical data of these hosts were collected, including average chondrule size, FeNi metal content, metamorphic grade, shock stage, and olivine and low-Ca pyroxene composition. These data and a proposed class were then submitted to the nomenclature committee of the Meteoritical Society for approval. 
Table 2.1: Meteorites marked with a (*) were classified for this study.

\begin{tabular}{|c|c|c|c|}
\hline Meteorite & Type & Inclusions & $\begin{array}{l}\text { CML or AMNH ID - } \\
\text { PTS \# }\end{array}$ \\
\hline NWA $8231^{*}$ & H4-6 & 8231-I1 & CML 0368-1 \\
\hline Tamdakht & $\mathrm{H} 5$ & $\begin{array}{l}\text { Tdk-I1 } \\
\text { Tdk-I2 }\end{array}$ & $\begin{array}{l}\text { CML 0646-1-1 } \\
\text { CML 0646-2-1 }\end{array}$ \\
\hline NWA $7873^{*}$ & H5-6 & 7873-I1 & CML 0615-2a \\
\hline Etter & $\mathrm{H} 6$ & Etr-I1 & AMNH 4462-1 \\
\hline Gunlock & L3. 6 & Glk-I1 & AMNH 4620-3 \\
\hline Khohar & L3. 6 & Khr-I1 & AMNH 4245-1 \\
\hline NWA $7869^{*}$ & L3.7 & 7869-I1, 7869-I2 & CML 0143-1 \\
\hline NWA $7872^{*}$ & L3.7 & $7872-\mathrm{I} 1$ & CML 0359-3 \\
\hline NWA 869 & L3-6 & 869-I1 & CML 0074-13c \\
\hline NWA $8141^{*}$ & L3-6 & 8141-I1 & CML 0014-1 \\
\hline Cynthiana & $\mathrm{L} 4$ & Cyn-I1 & AMNH 0369-1 \\
\hline Kramer Creek & $\mathrm{L} 4$ & $\mathrm{KrC}-\mathrm{I} 1$ & AMNH 4428-1 \\
\hline McKinney & $\mathrm{L} 4$ & McK-I1, McK-I2 & AMNH 0391-1 \\
\hline NWA $7870^{*}$ & $\mathrm{~L} 4$ & $7870-\mathrm{I} 1$ & CML 0173-3 \\
\hline NWA $7871^{*}$ & L6 & 7871-I1 & CML 0178-2 \\
\hline Palo Verde Mine & L6 & PVM-I1 & CML 0185-9b \\
\hline Ragland & LL3.4 & Rgl-I1 & AMNH 4825-3 \\
\hline Parnallee & LL3.6 & $\begin{array}{l}\text { Par-I1, Par-I2 } \\
\text { Par-I3 }\end{array}$ & $\begin{array}{l}\text { AMNH 1096-2 } \\
\text { CML 0524-3 }\end{array}$ \\
\hline Lut $005^{*}$ & LL3.7 & Lut-I1 & CML 0740-3 \\
\hline Richfield & LL3.7 & Rfd-I1 & AMNH 4887-1 \\
\hline NWA 4859 & LL5 & $\begin{array}{l}4859-\mathrm{I} 4 \\
4859-\mathrm{I} 18\end{array}$ & $\begin{array}{l}\text { CML 0272-14a } \\
\text { CML 0272-18 }\end{array}$ \\
\hline NWA $8232^{*}$ & LL5 & 8232-I1 & CML 0372-4 \\
\hline Oberlin & LL5 & Obl-I1 & AMNH 3832-1 \\
\hline
\end{tabular}


Polished thin sections of 28 inclusions, and one polished thick section, have been examined with OLM using a Leica DM 2500 petrographic microscope at Portland State University. Images were obtained using three different light modes: reflected, plane transmitted, and cross-polarized (see figure 2.1). Petrographic data such as texture, grain sizes and shapes were collected for the inclusions in order to facilitate comparisons. 


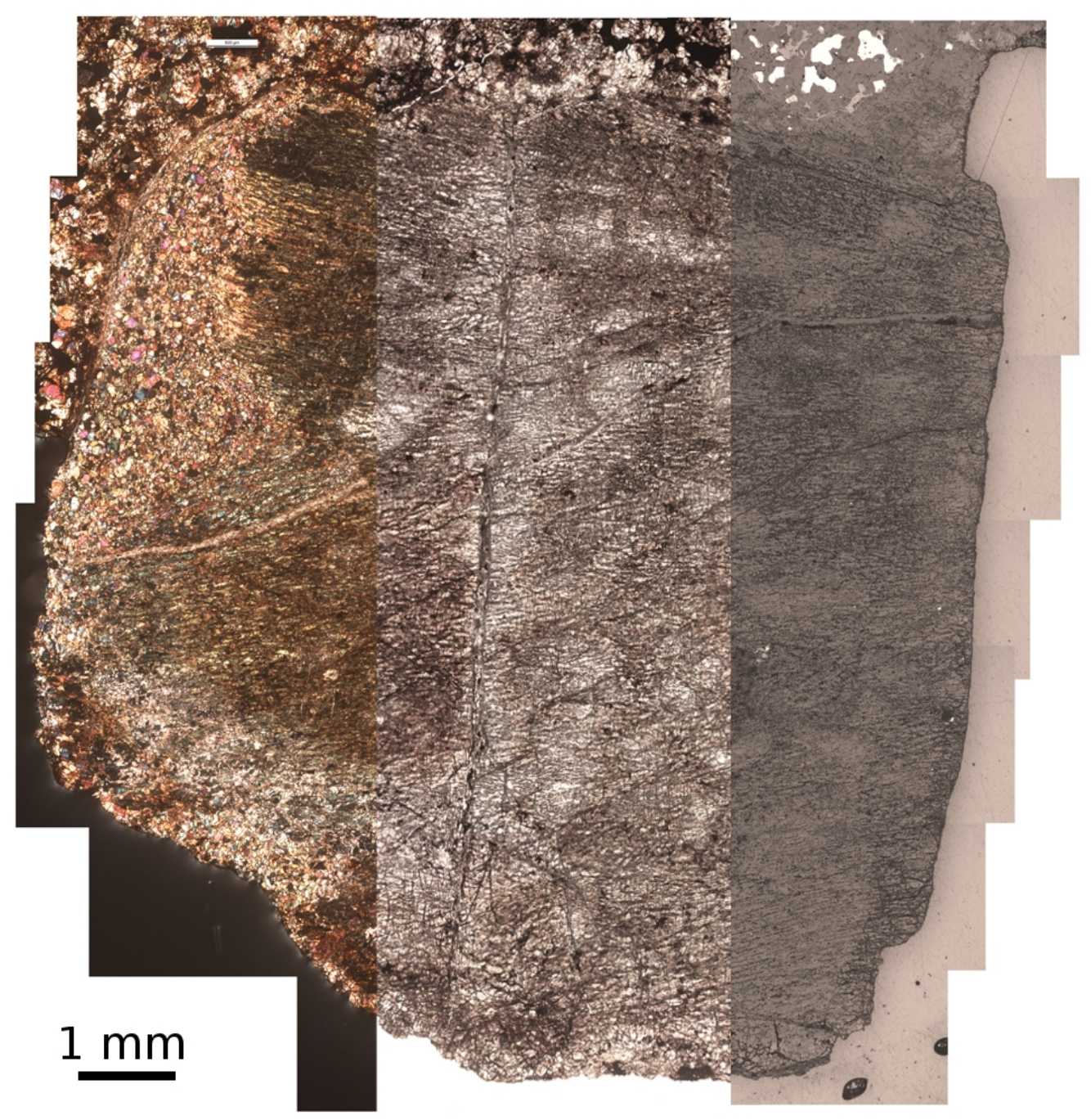

Figure 2.1: The three light modes used for collecting data: cross-polarized (left), plane transmitted (center) and reflected (right).

The inclusions were then investigated with scanning electron microscopy (SEM) on a Zeiss Sigma FE-VP SEM at Portland State University. Backscattered electron micrographs and false-color chemical phase maps were obtained in order to provide additional petrographic data (see figure 2.2). Phase compositions were obtained with the SEM using a silicon-drift energy dispersive X-ray (EDX) detector integrated with 
an Oxford Instruments AZtec X-ray analytical system. For collecting point spectra, the accelerating voltage used was $15 \mathrm{kV}$, and data was typically collected for 20 seconds. The elements $\mathrm{Na}, \mathrm{Mg}, \mathrm{Ca}, \mathrm{K}, \mathrm{Ti}, \mathrm{Cr}, \mathrm{Mn}, \mathrm{Fe}, \mathrm{S}$, and Ni were pre-defined; any others present were auto-detected. For BSE images, the scan size used was 2048 with a dwell time of $5 \mu \mathrm{s}$.

In addition, olivine crystals of 7 inclusions were investigated with electron microprobe analysis (EMPA), performed with a Cameca SX-100 electron microprobe located at Oregon State University, and remotely operated from Portland State. The instrument was operated at $15 \mathrm{kV}$ accelerating voltage, with a 10-50 nA sample current and a beam diameter of approximately $1 \mu \mathrm{m}$.

\subsection{Data Analysis}

The false-color phase maps were used to determine modal abundance of major phases in each inclusion (phases present at quantities $\geq 0.1$ volume percent). The maps were constructed by assigning unique colors to X-ray intensity maps of individual elements; each phase is thus a different color (see figure 2.2). The color variation is sensitive to subtle variations in chemistry; even zoning within phases can be quantified (see figure 2.3).

Photoshop and GIMP image-editing software programs were used to mask host material, isolating the inclusion (see figure 2.3). These maps were then processed with ColorInspector 3d, a plugin to the image processing software program ImageJ. ColorInspector 3d allows for each pixel in the image to be "binned" and counted by color. Additionally, the color depth of the image can be defined, which reduces the colors in an image to a specified, manageable number; this allows for each color "bin" 
to be assigned to a phase manually (see figure 2.3).

The majority of uncertainty in this method arises from some of the colors representing phase mixtures, fractures, or other areas that should be excluded from the count. For the most part, these were easily recognizable and subtracted. Ambiguous colors, which are either mixtures or single phases, were the largest source of error. This error was quantified by varying the number of color bins and taking repeated measurements; typically, this technique allowed for a determination of phase abundance to a precision of $\leq 2$ area percent.

Phase composition was determined by obtaining point spectra at the time of data collection, by extracting the data from the EDS map in post-processing, or both. The data in the EDS maps can be extracted either by selecting a specific area, or by utilizing an automated phase analysis function of the AZtec software. The latter is particularly useful in characterizing the minor phases. The data for each phase was averaged, with one standard deviation used to represent error. For silicates, chromite, and phosphate, oxygen was calculated by stoichiometry. Thresholding was enabled, with a sigma of one. Analyses of silicates were accepted if the ratio $(\mathrm{Mg}+\mathrm{Fe}) / \mathrm{Si})$ was 2.0 \pm 0.05 ; pyroxene analyses were accepted if $(\mathrm{Mg}+\mathrm{Fe}+\mathrm{Ca}) / \mathrm{Si}$ was $1.0 \pm 0.05$, and feldspar analyses were accepted if $(\mathrm{Na}+\mathrm{K}+\mathrm{Ca}) /(\mathrm{Al}+\mathrm{Si})$ was $0.25 \pm 0.03$.

Average phase composition was then combined with modal abundance to calculate bulk chemistry via modal reconstruction. For this, volume percent phase abundanceassumed to be equal to area percent- was normalized to 100 and converted to weight percent. Mineral densities used in this calculation are from Klein and Dutrow (2007). Errors from the modal abundance and the average phase composition were propagated to obtain an uncertainty for the final bulk chemistry. The error propagation equations 
used, for values $A \pm a$ and $B \pm b$, are:

$$
\begin{aligned}
\operatorname{err}(A \cdot B) & =\sqrt{(A \cdot b)^{2}+(B \cdot a)^{2}} \\
\operatorname{err}(A / B) & =\sqrt{\left(\frac{a}{A}\right)^{2}+\left(\frac{b}{B}\right)^{2}}
\end{aligned}
$$

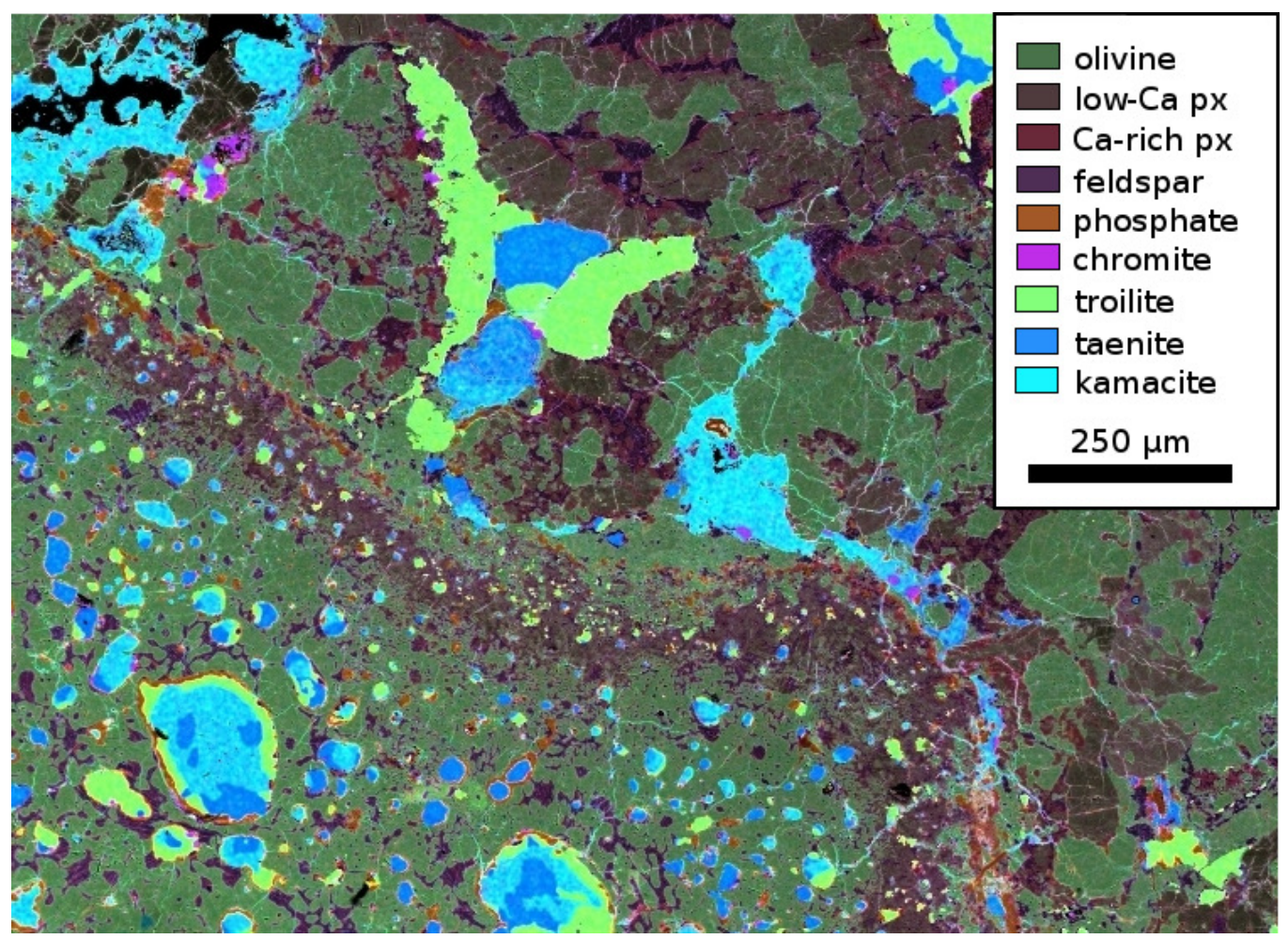

Figure 2.2: Example of the false-color phase maps that were obtained with the SEM. Assigning different colors to the various elements present allowed for each phase to be represented by a unique color or range of colors. 


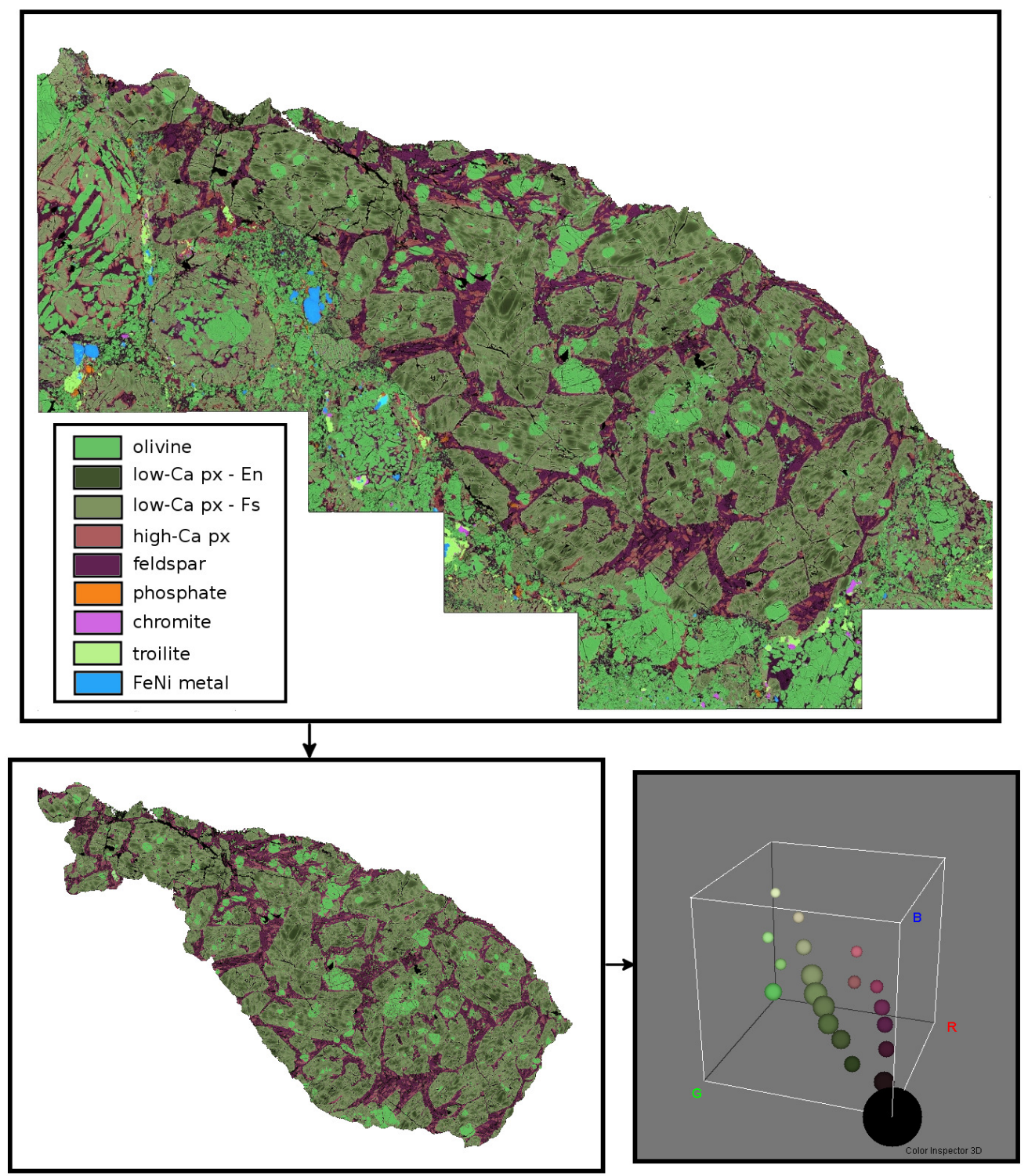

Figure 2.3: False-color EDS phase maps were collected with the SEM. Unique colors were assigned to individual elements, allowing the different phases to appear as different colors (top image). This map was masked to exclude host meteorite material, and the number of colors were reduced to a manageable number (bottom left image). This altered map was processed with the ImageJ plugin ColorInspector 3D, which "binned" the pixels based on colors (bottom right image. Black was used as the "masking" color, hence the large number of black pixels, but was changed to white for clarity in this figure). Each bin was then assigned to a phase manually. 


\section{Results}

A summary of results is presented here, with thorough petrographic and chemical data for each individual inclusion provided in appendix A. The 29 inclusions studied were, unsurprisingly, diverse. Nevertheless, some trends and similarities were observed, suggesting some commonalities.

Table 3.1: Overall geochemical characteristics and form of the inclusions, presented in order of host chemical type and increasing petrologic type. "Eq" refers to equilibrated, if both olivine and low-Ca pyroxene have $\sigma<1 \mathrm{~mol} \%$ in fayalite or ferrosilite content, respectively ( $\sigma=$ one standard deviation from average phase composition). "Comp. same as host" refers to whether or not $\mathrm{FeO}$ content of olivine and low-Ca pyroxene is approximately the same as the host meteorite (as reported by Brearley and Jones, 1998). Bulk trend refers to a generalized characterization for bulk composition; "unfrac" = roughly chondritic composition for lithophile elements, vapor frac $=$ vapor-fractionated composition, meaning showing a systematic deviation in CI-normalized abundances of lithophile elements with respect to $50 \%$ condensation temperature. For bulk chemistry of individual inclusions, see table 3.3 and appendix A. "Independent" refers to whether or not there is any indication that the inclusion interacted with its surroundings- e.g., a rim of different composition than the interior- or clear evidence of the inclusion crystallizing as a free-floating droplet.

\begin{tabular}{llllll}
\hline Inclusion & $\begin{array}{l}\text { Host } \\
\text { type }\end{array}$ & eq.? & $\begin{array}{l}\text { comp. same } \\
\text { as host? }\end{array}$ & bulk trend & independent? \\
\hline 8231-I1 & H4-6 & yes & yes & unfrac & no \\
Tdk-I1 & H5 & no & yes & unfrac* & yes, droplet \\
Tdk-I2 & H5 & no & yes & vapor frac & no \\
$7873-I 1$ & H5-6 & no & no - low & unfrac & no \\
Etr-I1 & H6 & yes & no - high & unfrac* & no \\
& & & & & no - low \\
Glk-I1 & L3.6 & no & unfrac & no \\
Khr-I1 & L3.6 & no & no - low & vapor frac & no \\
$7869-I 1$ & L3.7 & no & no - low & vapor frac & yes, droplet \\
continued on next page & & & & \\
\hline
\end{tabular}

*These inclusions are enriched or depleted in a feldspathic component but otherwise chondritic (see section 4.3.2) 
Table 3.1 continued

\begin{tabular}{|c|c|c|c|c|c|}
\hline Inclusion & $\begin{array}{l}\text { Host } \\
\text { type }\end{array}$ & eq.? & $\begin{array}{l}\text { comp. same } \\
\text { as host? }\end{array}$ & bulk trend & independent? \\
\hline 7869-I2 & L3.7 & no & yes & vapor frac & no \\
\hline 7872-I1 & L3.7 & no & no - low & vapor frac & yes, droplet \\
\hline 869-I1 & L3-6 & no & no - low & unfrac & no \\
\hline 8141-I1 & L3-6 & yes & yes & unfrac & yes \\
\hline Cyn-I1 & $\mathrm{L} 4$ & no & no - low & vapor frac & no \\
\hline $\mathrm{KrC}-\mathrm{I1}$ & $\mathrm{L} 4$ & no & no - low & vapor frac & yes, droplet \\
\hline McK-I1 & L4 & no & yes & vapor frac & no \\
\hline Mck-I2 & L4 & no & no - low & vapor frac & no \\
\hline 7870-I1 & $\mathrm{L} 4$ & yes & yes & unfrac* $^{*}$ & yes, droplet \\
\hline 7871-I1 & L6 & yes & yes & unfrac & no \\
\hline PVM-I1 & L6 & yes & yes & unfrac & no \\
\hline Rgl-I1 & LL3.4 & no & no - low & unfrac* & no \\
\hline Par-I1 & LL3. 6 & no & no - low & vapor frac & yes \\
\hline Par-I2 & LL3.6 & no & no - low & vapor frac & yes, droplet \\
\hline Par-I3 & LL3. 6 & yes & yes & unfrac & yes, droplet \\
\hline Lut-I1 & LL3.7 & no & no - low & vapor frac & yes, droplet \\
\hline Rfd-I1 & LL3.7 & no & no - low & vapor frac & yes, droplet \\
\hline 4859-I4 & LL5 & yes & no - low & unfrac* & yes \\
\hline 4859-I18 & LL5 & yes & yes & unfrac & yes \\
\hline 8232-I1 & LL5 & yes & yes & unfrac & yes \\
\hline Obl-I1 & LL5 & yes & yes & unfrac & no \\
\hline
\end{tabular}


Table 3.2: Physical properties of the inclusions. Apparent size is the average diameter of the inclusion in thin section; for inclusions that are clearly fragments, an original size is estimated (see appendix for detailed descriptions of individual inclusions). Estimated volume assumes that the inclusion is spherical and that its apparent size is its maximum diameter, and estimated mass assumes a density of $3.5 \mathrm{~g} / \mathrm{mm}^{3}$. Overall texture refers to the predominant texture of the inclusion. Abbreviations used for texture are: $\mathrm{mp}=$ microporphyritic, fgm = glassy or especially fine-grained mesostasis, $\mathrm{RP}=$ radial pyroxene, poik $=$ poikilitic, crypto $=$ cryptocrystalline. Average grain size is approximate and refers to phenocrysts only.

\begin{tabular}{|c|c|c|c|c|c|}
\hline Inclusion & $\begin{array}{l}\text { apparent } \\
\text { size }(\mathrm{mm})\end{array}$ & $\begin{array}{l}\text { estimated } \\
\mathrm{V}\left(\mathrm{mm}^{3}\right)\end{array}$ & $\begin{array}{l}\text { estimated } \\
\text { mass (g) }\end{array}$ & $\begin{array}{l}\text { overall } \\
\text { texture }\end{array}$ & $\begin{array}{l}\text { average } \\
\text { grain size } \\
(\mu \mathrm{m})\end{array}$ \\
\hline 8231-I1 & 18 & 3052 & 10.68 & poik & 400 \\
\hline Tdk-I1 & 4 & 33 & 0.12 & $\mathrm{RP}$ & $10 \times 100$ \\
\hline Tdk-I2 & 6 & 113 & 0.40 & $\mathrm{mp}$ & 500 \\
\hline 7873-I1 & 7 & 180 & 0.63 & $\mathrm{mp} / \mathrm{fgm}$ & 150 \\
\hline Etr-I1 & 9.5 & 449 & 1.57 & $\mathrm{mp}$ & 2000 \\
\hline Glk-I1 & 50 & 65417 & 228.96 & $\mathrm{mp} / \mathrm{fgm}$ & 100 \\
\hline Khr-I1 & 3.5 & 22 & 0.08 & $\mathrm{mp}$ & 150 \\
\hline 7872-I1 & 5 & 65 & 0.23 & $\mathrm{mp} / \mathrm{fgm}$ & 70 \\
\hline 7869-I1 & 3.5 & 22 & 0.08 & $\mathrm{mp}$ & 300 \\
\hline 7869-I2 & 4 & 33 & 0.12 & skeletal & $1000 \times 30$ \\
\hline 869-I1 & 30 & 14130 & 49.46 & $\mathrm{mp} / \mathrm{fgm}$ & 100 \\
\hline 8141-I1 & 3.4 & 21 & 0.07 & $\mathrm{mp}$ & 250 \\
\hline Cyn-I1 & 4.5 & 48 & 0.17 & $\mathrm{mp}$ & 100 \\
\hline KrC-I1 & 3.5 & 22 & 0.08 & crypto & 20 \\
\hline McK-I1 & 3.5 & 22 & 0.08 & $\mathrm{mp}$ & 100 \\
\hline McK-I2 & 3 & 14 & 0.05 & $\mathrm{mp}$ & 100 \\
\hline 7870-I1 & 5 & 65 & 0.23 & skeletal & $500 \times 20$ \\
\hline 7871-I1 & 9.5 & 449 & 1.57 & crypto & 30 \\
\hline PVM-I1 & 5 & 65 & 0.23 & $\mathrm{mp}$ & 200 \\
\hline
\end{tabular}

continued on next page 
Table 3.2 continued

\begin{tabular}{llllll}
\hline Inclusion & $\begin{array}{l}\text { apparent } \\
\text { size }(\mathrm{mm})\end{array}$ & $\begin{array}{l}\text { estimated } \\
\mathrm{V}\left(\mathrm{mm}^{3}\right)\end{array}$ & $\begin{array}{l}\text { estimated } \\
\text { mass }(\mathrm{g})\end{array}$ & $\begin{array}{l}\text { overall } \\
\text { texture }\end{array}$ & $\begin{array}{l}\text { average } \\
\text { grain size } \\
(\mu \mathrm{m})\end{array}$ \\
\hline Rgl-I1 & 12 & 904 & 3.17 & $\mathrm{mp} /$ fgm & 100 \\
Par-I1 & 10 & 523 & 1.83 & $\mathrm{mp}$ & 250 \\
Par-I2 & 3.2 & 17 & 0.06 & $\mathrm{mp} /$ fgm & 500 \\
Par-I3 & 3.5 & 22 & 0.08 & crypto & $1 \times 10$ \\
Lut-I1 & 8 & 268 & 0.94 & skeletal & $100 \times 20$ \\
Rfd-I1 & 5.5 & 87 & 0.30 & skeletal & $100 \times 20$ \\
4859-I4 & 16 & 2144 & 7.50 & $\mathrm{mp}$ & 500 \\
4859-I18 & 18 & 3052 & 10.68 & $\mathrm{mp} /$ fgm & 100 \\
8232-I1 & 7.5 & 221 & 0.77 & $\mathrm{mp}$ & 100 \\
Obl-I1 & 6 & 113 & 0.40 & $\mathrm{mp}$ & 1000 \\
\hline
\end{tabular}

Modal abundances were highly variable between the inclusions. Most were assemblages of olivine, two or more pyroxenes, and feldspar, with phosphates, chromite, sulfide, and FeNi metal as accessory minerals. Some were not fully crystalline and contained a glassy mesostasis. Other phases observed were spinel, sodalite, and ilmenite. Figure 3.1 and table 3.3 contain all modal abundance data. 


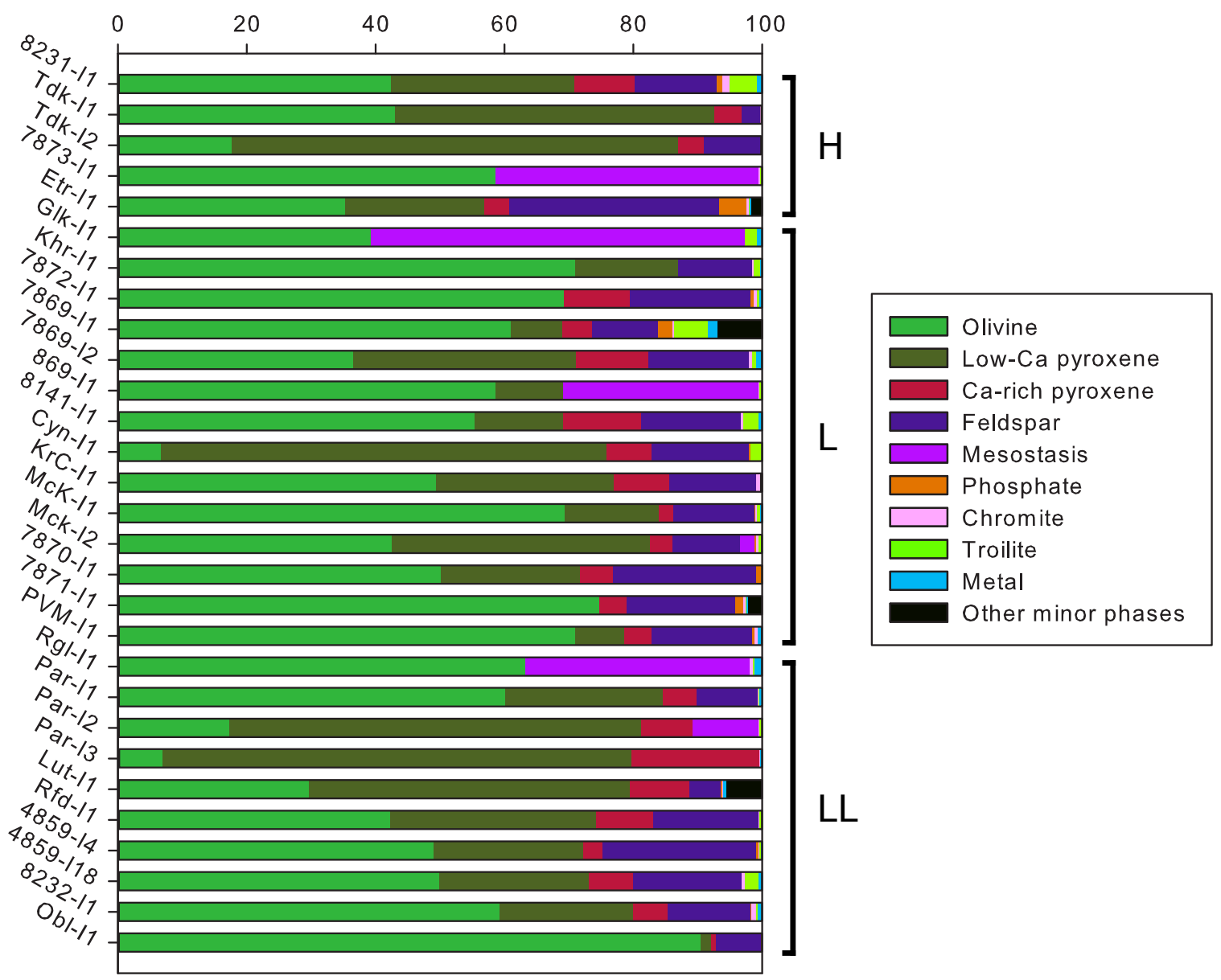

Figure 3.1: Visual representation of the modal abundance of each inclusion. Inclusions are presented in the same order as tables 2.1, 3.1, etc, which is by host type and metamorphic grade. See table 3.3 for numeric data including errors.

Table 3.3: Modal abundance of all inclusions, given in volume \%. Abbreviations used are: $\mathrm{px}=$ pyroxene, $\mathrm{Cpx}=$ Ca-pyroxene, $\mathrm{ap}=$ aluminous pyroxene, $\mathrm{il}=$ ilmenite, $\mathrm{so}=$ sodalite, and $\mathrm{sp}=$ spinel.

\begin{tabular}{llllll}
\hline Inclusion & Olivine & Low-Ca px & Cpx & Feldspar & Mesostasis \\
\hline & & & & & \\
$8231-\mathrm{I} 1$ & $42.6 \pm 3.7$ & $27.6 \pm 3.2$ & $9.4 \pm 2.5$ & $12.7 \pm 2.2$ & - \\
Tdk-I1 & $43.1 \pm 2.1$ & $49.9 \pm 2.8$ & $4.1 \pm 0.4$ & $3 \pm 0.4$ & - \\
continued on next page & & & &
\end{tabular}




\begin{tabular}{llllll}
\hline Inclusion & Olivine & Low-Ca px & Cpx & Feldspar & Mesostasis \\
\hline Tdk-I2 & $17.8 \pm 0.05$ & $69.4 \pm 0.7$ & $4 \pm 0.05$ & $8.9 \pm 0.8$ & - \\
7873-I1 & $58.7 \pm 7.6$ & - & - & - & $40.7 \pm 1.0$ \\
Etr-I1 & $34.9 \pm 3.1$ & $19.5 \pm 1.2$ & $3.85 \pm 0.55$ & $30.8 \pm 1.7$ & - \\
Glk-I1 & $39.1 \pm 0.16$ & - & - & - & $58.0 \pm 2.9$ \\
Khr-I1 & $69.9 \pm 0.8$ & $15.6 \pm 0.9$ & - & $11.4 \pm 0.7$ & - \\
$7872-I 1$ & $69.1 \pm 2.8$ & - & $10.2 \pm 0.7$ & $18.8 \pm 2.8$ & - \\
$7869-I 1$ & $61 \pm 5$ & $8 \pm 2.2$ & $4.6 \pm 0.2$ & $10.3 \pm 0.2$ & - \\
$7869-I 2$ & $36.2 \pm 1.3$ & $34.4 \pm 0.5$ & $11.2 \pm 2.3$ & $15.5 \pm 1$ & - \\
869-I1 & $58.8 \pm 3.5$ & $10.5 \pm 1.8$ & - & - & $30.3 \pm 3.5$ \\
8141-I1 & $55 \pm 0.3$ & $13.7 \pm 2.1$ & $12.1 \pm 1$ & $15.3 \pm 1.4$ & - \\
Cyn-I1 & $6.6 \pm 0.5$ & $68.4 \pm 6.6$ & $6.8 \pm 0.3$ & $15 \pm 0.4$ & - \\
KrC-I1 & $49.3 \pm 2.1$ & $27.5 \pm 0.2$ & $8.6 \pm 1.4$ & $13.5 \pm 2.1$ & - \\
McK-I1 & $69.3 \pm 0.9$ & $14.5 \pm 0.4$ & $2.2 \pm 0.4$ & $12.6 \pm 0.9$ & - \\
Mck-I2 & $43.4 \pm 0.3$ & $41 \pm 1.6$ & $3.6 \pm 1.6$ & $10.7 \pm 0.9$ & $2.3 \pm 0.1$ \\
7870-I1 & $50.4 \pm 3$ & $21.7 \pm 3.2$ & $5.2 \pm 2$ & $22.4 \pm 1.7$ & - \\
7871-I1 & $73.9 \pm 1.6$ & - & $4.3 \pm 0.9$ & $16.6 \pm 2.5$ & - \\
PVM-I1 & $71 \pm 2.3$ & $7.5 \pm 1.6$ & $4.3 \pm 1.4$ & $15.6 \pm 1.1$ & - \\
Rgl-I1 & $62.7 \pm 8$ & - & - & - & 3 \\
Par-I1 & $60 \pm 2.9$ & $24.5 \pm 1.1$ & $4.9 \pm 0.2$ & $9.8 \pm 1.6$ & - \\
Par-I2 & $17.3 \pm 0.35$ & $63.9 \pm 5.1$ & $8 \pm 0.2$ & - & $10.1 \pm 0.15$ \\
Par-I3 & $6.9 \pm 1.3$ & $72.6 \pm 2.1$ & $19.5 \pm 2$ & - & - \\
Lut-I1 & $29.9 \pm 2.9$ & $50.35 \pm 1.8$ & $9.4 \pm 0.6$ & $4.9 \pm 2.6$ & - \\
Rfd-I1 & $42.9 \pm 0.6$ & $32.4 \pm 0.2$ & $9.1 \pm 2.3$ & $16.6 \pm 1.5$ & - \\
$4859-I 4$ & $49.1 \pm 1.2$ & $23.3 \pm 1.6$ & $3.1 \pm 0.1$ & $23.9 \pm 1.5$ & - \\
4859-I18 & $50.2 \pm 2.9$ & $23.4 \pm 0.9$ & $6.9 \pm 1.9$ & $16.9 \pm 2.4$ & - \\
$8232-I 1$ & $59.8 \pm 2.3$ & $20.9 \pm 2.9$ & $5.4 \pm 2.4$ & $12.9 \pm 1$ & - \\
Obl-I1 & $89.2 \pm 1.1$ & $1.5 \pm 0.8$ & $0.8 \pm 0.1$ & $6.8 \pm 0.8$ & - \\
\hline
\end{tabular}


3 Results

Table 3.3 continued

\begin{tabular}{|c|c|c|c|c|c|}
\hline Inclusion & Chromite & Troilite & $\mathrm{FeNi}$ & Phosphate & Other \\
\hline $8231-\mathrm{I} 1$ & $0.74 \pm 0.5$ & $4.3 \pm 2.7$ & $0.76 \pm 0.1$ & $1.1 \pm 0.2$ & \\
\hline Tdk-I1 & $0.07 \pm 0.01$ & $0.14 \pm 0.02$ & $0.02 \pm 0.01$ & - & \\
\hline Tdk-I2 & $0.11 \pm 0.01$ & - & $0.094 \pm 0.01$ & $0.044 \pm 0.01$ & $\mathrm{il}-0.01 \pm 0.01$ \\
\hline 7873-I1 & $0.1 \pm 0.01$ & $0.4 \pm 0.1$ & $0.1 \pm 0.1$ & - & \\
\hline Etr-I1 & $0.43 \pm 0.12$ & $0.115 \pm 0.01$ & $0.11 \pm 0.01$ & $4.1 \pm 0.1$ & ap $-1.8 \pm 0.1$ \\
\hline Glk-I1 & $0.05 \pm 0.01$ & $1.8 \pm 0.01$ & $0.82 \pm 0.05$ & - & \\
\hline Khr-I1 & $0.16 \pm 0.01$ & $0.86 \pm 0.1$ & $0.5 \pm 0.06$ & - & \\
\hline $7872-\mathrm{I} 1$ & $0.58 \pm 0.02$ & $0.33 \pm 0.2$ & $0.3 \pm 0.08$ & $0.35 \pm 0.06$ & il $-0.16 \pm 0.005$ \\
\hline 7869-I1 & $0.21 \pm 0.02$ & $5.4 \pm 1.1$ & $6.8 \pm 1.8$ & $2.3 \pm 0.8$ & $\mathrm{sp}-1.5 \pm 0.1$ \\
\hline 7869-I2 & $0.42 \pm 0.01$ & $0.7 \pm 0.03$ & $0.9 \pm 0.01$ & - & \\
\hline 869-I1 & $0.09 \pm 0.06$ & $0.39 \pm 0.1$ & $0.09 \pm 0.07$ & - & ap $-0.03 \pm 0.01$ \\
\hline 8141-I1 & $0.43 \pm 0.1$ & $2.3 \pm 0.2$ & $0.6 \pm 0.2$ & - & \\
\hline Cyn-I1 & $0.07 \pm 0.01$ & $1.9 \pm 0.05$ & $0.01 \pm 0.01$ & $0.04 \pm 0.01$ & il $-0.01 \pm 0.01$ \\
\hline $\mathrm{KrC}-\mathrm{I} 1$ & $0.57 \pm 0.1$ & $0.15 \pm 0.03$ & $0.07 \pm 0.01$ & - & il $-0.16 \pm 0.03$ \\
\hline McK-I1 & $0.26 \pm 0.1$ & $0.62 \pm 0.2$ & $0.2 \pm 0.01$ & $0.13 \pm 0.05$ & \\
\hline Mck-I2 & $0.4 \pm 0.2$ & $0.5 \pm 0.3$ & $0.1 \pm 0.01$ & $0.14 \pm 0.01$ & \\
\hline 7870-I1 & $0.06 \pm 0.02$ & - & $0.09 \pm 0.02$ & $0.75 \pm 0.11$ & il - $0.01 \pm 0.01$ \\
\hline 7871-I1 & $0.56 \pm 0.01$ & $0.15 \pm 0.01$ & $0.05 \pm 0.01$ & $1.1 \pm 0.1$ & \\
\hline PVM-I1 & $0.5 \pm 0.09$ & $0.02 \pm 0$ & $0.67 \pm 0.11$ & $0.41 \pm 0.02$ & \\
\hline Rgl-I1 & $0.41 \pm 0.07$ & $0.23 \pm 0.15$ & $0.94 \pm 0.5$ & - & il $-0.2 \pm 0.07$ \\
\hline Par-I1 & $0.06 \pm 0.01$ & $0.12 \pm 0.01$ & $0.35 \pm 0.01$ & - & il $-0.2 \pm 0.01$ \\
\hline Par-I2 & $0.05 \pm 0.01$ & $0.53 \pm 0.1$ & $0.2 \pm 0.03$ & - & \\
\hline Par-I3 & $0.13 \pm 0.03$ & - & $0.61 \pm 0.49$ & - & \\
\hline Lut-I1 & $0.05 \pm 0.01$ & - & $0.67 \pm 0.01$ & $0.1 \pm 0.01$ & $\begin{array}{l}\text { so }-5.6 \pm 1.7 \\
\text { il - } 0.12 \pm 0.05\end{array}$ \\
\hline Rfd-I1 & $0.07 \pm 0.01$ & $0.3 \pm 0.07$ & $0.09 \pm 0.04$ & - & il $-0.1 \pm 0.005$ \\
\hline 4859-I4 & $0.12 \pm 0.05$ & $0.27 \pm 0.01$ & $0.2 \pm 0.01$ & $0.29 \pm 0.04$ & il $-0.01 \pm 0.01$ \\
\hline
\end{tabular}

continued on next page 
Table 3.3 continued

\begin{tabular}{llllll}
\hline Inclusion & Chromite & Troilite & FeNi & Phosphate & Other \\
\hline 4859-I18 & $0.36 \pm 0.13$ & $2.7 \pm 0.6$ & $0.17 \pm 0.05$ & $0.08 \pm 0.01$ & il $-0.028 \pm 0.01$ \\
8232-I1 & $0.52 \pm 0.1$ & $0.29 \pm 0.04$ & $0.84 \pm 0.02$ & $0.22 \pm 0.01$ & \\
Obl-I1 & $0.14 \pm 0.01$ & - & $0.008 \pm 0.01$ & $0.1 \pm 0.01$ & \\
\hline
\end{tabular}

The bulk chemistry of the inclusions is also fairly diverse. Table 3.3 contains oxide wt\% data for all inclusions. See appendix A for CI-normalized plots of bulk chemical data for each inclusion.

Table 3.3: Bulk chemistry of all inclusions, given in Wt \%. Fe (S) refers to Fe in a sulfide phase, Fe (M) refers to Fe in a metallic phase. Totals are 100 within rounding error.

\begin{tabular}{lcccccc}
\hline Oxide & $8231-\mathrm{I} 1$ & $\mathrm{Tdk}-\mathrm{I} 1$ & $\mathrm{Tdk}-\mathrm{I} 2$ & $7873-\mathrm{I} 1$ & Etr-I1 & Glk-I1 \\
\hline $\mathrm{SiO}_{2}$ & $43.4 \pm 2.9$ & $47.9 \pm 1.8$ & $51 \pm 0.8$ & $46.6 \pm 2.4$ & $48.3 \pm 2.2$ & $47.7 \pm 2.2$ \\
$\mathrm{TiO}_{2}$ & $0.1 \pm 0$ & $0.1 \pm 0.1$ & $0.1 \pm 0.1$ & $0.1 \pm 0.1$ & $0.2 \pm 0$ & $0.2 \pm 0.1$ \\
$\mathrm{Al}_{2} \mathrm{O}_{3}$ & $2.2 \pm 0.4$ & $1.1 \pm 0.2$ & $4.9 \pm 0.6$ & $2.5 \pm 0.1$ & $6.7 \pm 0.5$ & $2.9 \pm 0.2$ \\
$\mathrm{Cr}_{2} \mathrm{O}_{3}$ & $0.9 \pm 0.4$ & $0.4 \pm 0.1$ & $0.5 \pm 0.2$ & $0.8 \pm 0.2$ & $0.4 \pm 0.1$ & $0.8 \pm 0.2$ \\
$\mathrm{FeO}$ & $12.8 \pm 0.9$ & $13 \pm 0.6$ & $10.6 \pm 0.5$ & $15.4 \pm 1.2$ & $12.9 \pm 0.8$ & $14.1 \pm 0.9$ \\
$\mathrm{MnO}$ & $0.4 \pm 0$ & $0.4 \pm 0.1$ & $0.4 \pm 0.1$ & $0.5 \pm 0.1$ & $0.3 \pm 0$ & $0.5 \pm 0.2$ \\
$\mathrm{MgO}$ & $27.8 \pm 1.9$ & $34.4 \pm 1.3$ & $28.5 \pm 0.7$ & $29.6 \pm 2.6$ & $22 \pm 1.4$ & $27.4 \pm 1.1$ \\
$\mathrm{CaO}$ & $2.6 \pm 0.5$ & $2.2 \pm 0.3$ & $3.5 \pm 0.5$ & $2.3 \pm 0.2$ & $3.6 \pm 0.2$ & $2.5 \pm 0.2$ \\
$\mathrm{Na} 2 \mathrm{O}$ & $1 \pm 0.2$ & $0.2 \pm 0$ & $0.2 \pm 0$ & $1 \pm 0.1$ & $2.8 \pm 0.3$ & $0.8 \pm 0.3$ \\
$\mathrm{~K}_{2} \mathrm{O}$ & $0.2 \pm 0.1$ & $<0.1$ & $<0.1$ & $0.1 \pm 0$ & $0.5 \pm 0.1$ & $0.1 \pm 0$ \\
$\mathrm{P}_{2} \mathrm{O} 5$ & $0.5 \pm 0.1$ & $<0.1$ & $<0.1$ & $<0.1$ & $1.7 \pm 0.1$ & $<0.1$ \\
$\mathrm{~S}$ & $2.2 \pm 1.4$ & $0.1 \pm 0$ & $<0.1$ & $0.3 \pm 0.1$ & $0.1 \pm 0$ & $0.6 \pm 0.1$ \\
$\mathrm{Fe}(\mathrm{S})$ & $4 \pm 2.5$ & $0.1 \pm 0$ & $<0.1$ & $0.4 \pm 0.1$ & $0.1 \pm 0$ & $<0.1$ \\
$\mathrm{Fe}(\mathrm{M})$ & $1.5 \pm 0.2$ & $<0.1$ & $0.2 \pm 0$ & $0.2 \pm 0.2$ & $0.2 \pm 0$ & $2.1 \pm 0$ \\
$\mathrm{Ni}$ & $0.3 \pm 0.1$ & $<0.1$ & $<0.1$ & $<0.1$ & $<0.1$ & $0.2 \pm 0$ \\
& & & & & & \\
\hline
\end{tabular}

continued on next page 
3 Results

Table 3.3 continued

\begin{tabular}{|c|c|c|c|c|c|c|}
\hline Oxide & Khr-I1 & $7872-\mathrm{I} 1$ & 7869-I1 & 7869-I2 & McK-I2 & 7870-I1 \\
\hline $\mathrm{SiO}_{2}$ & $41.1 \pm 0.7$ & $41.2 \pm 1.8$ & $31.8 \pm 1.7$ & $46.7 \pm 1.3$ & $45.5 \pm 1.3$ & $47.2 \pm 2.6$ \\
\hline $\mathrm{TiO}_{2}$ & $0.1 \pm 0$ & $0.4 \pm 0.1$ & $0.1 \pm 0$ & $<0.1$ & $0.1 \pm 0.1$ & $0.2 \pm 0$ \\
\hline $\mathrm{Al}_{2} \mathrm{O}_{3}$ & $3.7 \pm 0.2$ & $4.8 \pm 0.4$ & $3.6 \pm 0.2$ & $4.2 \pm 0.3$ & $3.7 \pm 0.3$ & $3.9 \pm 0.3$ \\
\hline $\mathrm{Cr}_{2} \mathrm{O}_{3}$ & $0.2 \pm 0$ & $0.6 \pm 0.1$ & $0.2 \pm 0$ & $0.7 \pm 0.1$ & $0.8 \pm 0.2$ & $0.3 \pm 0$ \\
\hline $\mathrm{FeO}$ & $15.1 \pm 1.3$ & $16.7 \pm 0.7$ & $11.4 \pm 1$ & $13.1 \pm 0.7$ & $15.6 \pm 0.7$ & $15.8 \pm 0.9$ \\
\hline $\mathrm{MnO}$ & $0.3 \pm 0.1$ & $0.4 \pm 0$ & $0.2 \pm 0$ & $0.4 \pm 0$ & $0.4 \pm 0.2$ & $0.4 \pm 0$ \\
\hline $\mathrm{MgO}$ & $34.2 \pm 0.9$ & $30.3 \pm 1.2$ & $27.1 \pm 1.6$ & $27.8 \pm 0.8$ & $30.3 \pm 0.8$ & $27.7 \pm 1.6$ \\
\hline $\mathrm{CaO}$ & $2.6 \pm 0.2$ & $3.5 \pm 0.3$ & $3 \pm 0.3$ & $3.1 \pm 0.4$ & $2.2 \pm 0.2$ & $2.1 \pm 0.5$ \\
\hline $\mathrm{Na}_{2} \mathrm{O}$ & $0.2 \pm 0.1$ & $0.9 \pm 0.2$ & $0.3 \pm 0$ & $0.7 \pm 0.1$ & $0.4 \pm 0$ & $1.7 \pm 0.1$ \\
\hline $\mathrm{K}_{2} \mathrm{O}$ & $<0.1$ & $0.1 \pm 0$ & $<0.1$ & $<0.1$ & $<0.1$ & $0.2 \pm 0$ \\
\hline $\mathrm{P}_{2} \mathrm{O}_{5}$ & $<0.1$ & $0.1 \pm 0$ & $0.8 \pm 0.3$ & $<0.1$ & $<0.1$ & $0.3 \pm 0$ \\
\hline S & $0.5 \pm 0.1$ & $0.2 \pm 0.1$ & $2.7 \pm 0.6$ & $0.4 \pm 0$ & & $<0.1$ \\
\hline $\mathrm{Fe}(\mathrm{S})$ & $0.8 \pm 0.1$ & $0.3 \pm 0.2$ & $4.4 \pm 0.9$ & $0.6 \pm 0$ & $0.5 \pm 0.3$ & $<0.1$ \\
\hline $\mathrm{Fe}(\mathrm{M})$ & $1.1 \pm 0.1$ & & $12.3 \pm 2.5$ & & $0.2 \pm 0$ & $0.2 \pm 0$ \\
\hline $\mathrm{Ni}$ & $0.1 \pm 0$ & $0.1 \pm 0$ & $2.3 \pm 0.6$ & $0.1 \pm 0$ & $<0.1$ & $<0.1$ \\
\hline Oxide & & & & & & \\
\hline $\mathrm{SiO}_{2}$ & $41.6 \pm 1.6$ & & $43.6 \pm 2.8$ & & $51.6 \pm 2$ & $47.3 \pm 1.5$ \\
\hline $\mathrm{TiO}_{2}$ & $<0.1$ & $0.1 \pm 0$ & $0.3 \pm 0.1$ & $0.2 \pm 0$ & $0.1 \pm 0$ & $0.2 \pm 0$ \\
\hline $\mathrm{Al}_{2} \mathrm{O}_{3}$ & $2.9 \pm 0.4$ & $2.7 \pm 0.2$ & $3.6 \pm 0.4$ & $3.3 \pm 0.5$ & $1.8 \pm 0.1$ & $2.8 \pm 0.2$ \\
\hline $\mathrm{Cr}_{2} \mathrm{O}_{3}$ & $0.5 \pm 0$ & $0.5 \pm 0.1$ & $0.5 \pm 0.1$ & $0.4 \pm 0$ & $0.7 \pm 0.1$ & $0.7 \pm 0.1$ \\
\hline $\mathrm{FeO}$ & $19.1 \pm 0.5$ & $18.6 \pm 0.7$ & $16.6 \pm 1.6$ & $15.8 \pm 0.8$ & $14.8 \pm 1$ & $17.6 \pm 0.6$ \\
\hline $\mathrm{MnO}$ & $0.4 \pm 0$ & $0.4 \pm 0.1$ & $0.3 \pm 0.2$ & $0.3 \pm 0$ & $0.5 \pm 0$ & $0.4 \pm 0.1$ \\
\hline $\mathrm{MgO}$ & $31.2 \pm 0.7$ & $30.9 \pm 1.1$ & $27 \pm 2.6$ & $32.8 \pm 1.1$ & $26.8 \pm 1.2$ & $26.5 \pm 0.9$ \\
\hline $\mathrm{CaO}$ & $2 \pm 0.3$ & $1.4 \pm 0.3$ & $2.8 \pm 0.4$ & $3 \pm 0.3$ & $1.8 \pm 0.2$ & $2.7 \pm 0.3$ \\
\hline $\mathrm{Na}_{2} \mathrm{O}$ & $1.2 \pm 0.2$ & $1.1 \pm 0.1$ & $1.9 \pm 0.2$ & $0.1 \pm 0$ & $0.7 \pm 0$ & $0.2 \pm 0.1$ \\
\hline $\mathrm{K}_{2} \mathrm{O}$ & $0.1 \pm 0$ & $0.1 \pm 0$ & $0.3 \pm 0.3$ & $<0.1$ & $<0.1$ & $0.1 \pm 0$ \\
\hline $\mathrm{P}_{2} \mathrm{O}_{5}$ & $0.4 \pm 0.1$ & $0.2 \pm 0$ & $0.5 \pm 0.1$ & $<0.1$ & $<0.1$ & $<0.1$ \\
\hline
\end{tabular}

continued on next page 
Table 3.3 continued

\begin{tabular}{|c|c|c|c|c|c|c|}
\hline S & $0.1 \pm 0$ & $<0.1$ & $0.1 \pm 0.1$ & $0.1 \pm 0$ & $0.3 \pm 0.1$ & $<0.1$ \\
\hline $\mathrm{Fe}(\mathrm{S})$ & $0.1 \pm 0$ & $<0.1$ & $0.2 \pm 0.1$ & $0.1 \pm 0$ & $0.5 \pm 0.1$ & $<0.1$ \\
\hline $\mathrm{Fe}(\mathrm{M})$ & $0.2 \pm 0$ & $1.4 \pm 0.2$ & $2.1 \pm 1.1$ & $0.7 \pm 0$ & $0.4 \pm 0$ & $1.2 \pm 0.7$ \\
\hline $\mathrm{Ni}$ & $<0.1$ & $0.2 \pm 0.1$ & $0.1 \pm 0.1$ & $0.2 \pm 0$ & $<0.1$ & $0.3 \pm 0.2$ \\
\hline Oxide & Lut-I1 & Rfd-I1 & 4859-I4 & 4859-I18 & 8232-I1 & Obl-I1 \\
\hline $\mathrm{SiO}_{2}$ & $48.5 \pm 1.7$ & $46.7 \pm 1.3$ & $46.7 \pm 1.3$ & $44 \pm 2$ & $43 \pm 2.2$ & $39.4 \pm 0.8$ \\
\hline $\mathrm{TiO}_{2}$ & $0.2 \pm 0.1$ & $0.2 \pm 0$ & $0.1 \pm 0$ & $0.1 \pm 0$ & $0.1 \pm 0$ & $<0.1$ \\
\hline $\mathrm{Al}_{2} \mathrm{O}_{3}$ & $3.8 \pm 0.9$ & $5.5 \pm 0.4$ & $4.5 \pm 0.3$ & $3 \pm 0.4$ & $2.3 \pm 0.2$ & $1.1 \pm 0.1$ \\
\hline $\mathrm{Cr}_{2} \mathrm{O}_{3}$ & $0.4 \pm 0.2$ & $0.5 \pm 0.1$ & $0.2 \pm 0.1$ & $0.4 \pm 0.1$ & $0.5 \pm 0.2$ & $0.1 \pm 0.1$ \\
\hline $\mathrm{FeO}$ & $9.9 \pm 0.9$ & $10 \pm 0.5$ & $16.9 \pm 0.4$ & $18.5 \pm 0.9$ & $20 \pm 0.8$ & $24.2 \pm 0.5$ \\
\hline $\mathrm{MnO}$ & $0.2 \pm 0.1$ & $0.2 \pm 0$ & $0.3 \pm 0.1$ & $0.4 \pm 0$ & $0.4 \pm 0$ & $0.4 \pm 0$ \\
\hline $\mathrm{MgO}$ & $31.3 \pm 1.2$ & $31.8 \pm 0.6$ & $26.8 \pm 0.7$ & $25.9 \pm 1.1$ & $28.3 \pm 1.2$ & $33.7 \pm 0.6$ \\
\hline $\mathrm{CaO}$ & $2.9 \pm 0.7$ & $4.2 \pm 0.5$ & $1.5 \pm 0.1$ & $2.1 \pm 0.4$ & $1.7 \pm 0.5$ & $0.4 \pm 0$ \\
\hline $\mathrm{Na}_{2} \mathrm{O}$ & $0.8 \pm 0.2$ & $0.2 \pm 0$ & $1.9 \pm 0.1$ & $1.2 \pm 0.2$ & $0.9 \pm 0.1$ & $0.5 \pm 0.1$ \\
\hline $\mathrm{K}_{2} \mathrm{O}$ & $0.1 \pm 0.1$ & $<0.1$ & $0.1 \pm 0$ & $0.1 \pm 0$ & $0.1 \pm 0$ & $<0.1$ \\
\hline $\mathrm{P}_{2} \mathrm{O}_{5}$ & $<0.1$ & $<0.1$ & $0.1 \pm 0$ & $0.1 \pm 0.1$ & $0.1 \pm 0$ & $<0.1$ \\
\hline S & $<0.1$ & $0.2 \pm 0$ & $0.1 \pm 0$ & $1.3 \pm 0.3$ & $0.1 \pm 0$ & $<0.1$ \\
\hline $\mathrm{Fe}(\mathrm{S})$ & $<0.1$ & $0.3 \pm 0.1$ & $0.3 \pm 0$ & $2.4 \pm 0.6$ & $0.3 \pm 0$ & $<0.1$ \\
\hline $\mathrm{Fe}(\mathrm{M})$ & $1.5 \pm 0$ & $0.2 \pm 0.1$ & $0.3 \pm 0$ & $0.3 \pm 0.1$ & $1.4 \pm 0.1$ & $<0.1$ \\
\hline $\mathrm{Ni}$ & $0.1 \pm 0$ & $<0.1$ & $0.2 \pm 0$ & $0.2 \pm 0$ & $0.6 \pm 0$ & $<0.1$ \\
\hline
\end{tabular}

Average phase compositions for olivine, low-Ca pyroxene, clinopyroxene, and feldspar are presented in figures 3.2 and 3.3, with full data, including error, in tables 3.4, 3.5, and 3.6, respectively. Again there was diversity, with some inclusions having phase compositions similar to their host meteorites, and some being entirely different. 


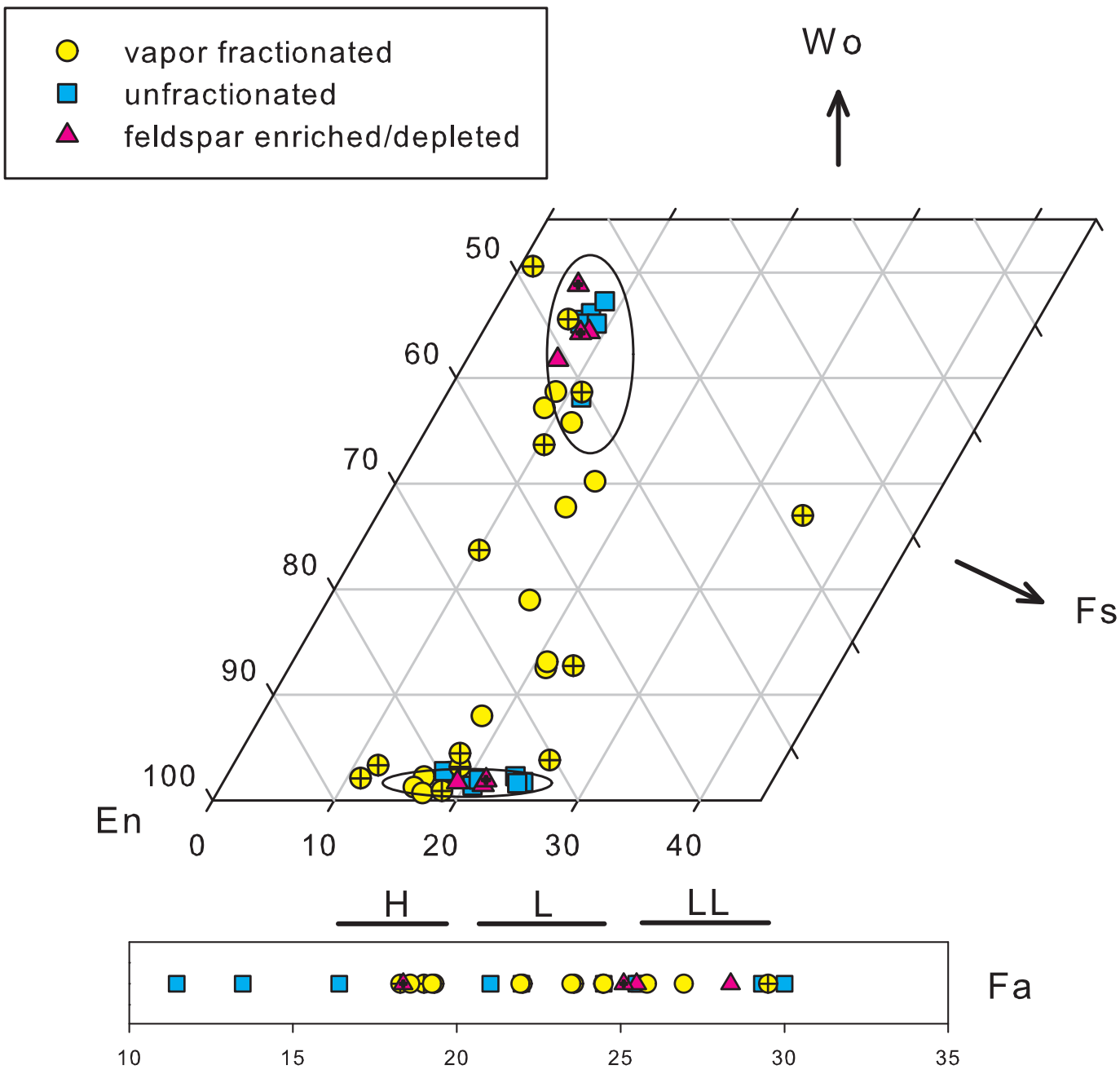

Figure 3.2: Average olivine and pyroxene phase composition (mol \%) for all inclusions. Wo, Fs, and En stand for wollastonite, ferrosilite, and enstatite, respectively. Ellipses show average equilibrated O chondrite (H4-6, L4-6, and LL4-6) pyroxene, from Brearley and Jones (1998). Crossed symbols are inclusions that are droplet-formed. Pyroxene of the vapor-fractionated inclusions is richer in $\mathrm{Mg}$ than those with a chondritic composition, though the same is not true for olivine. 


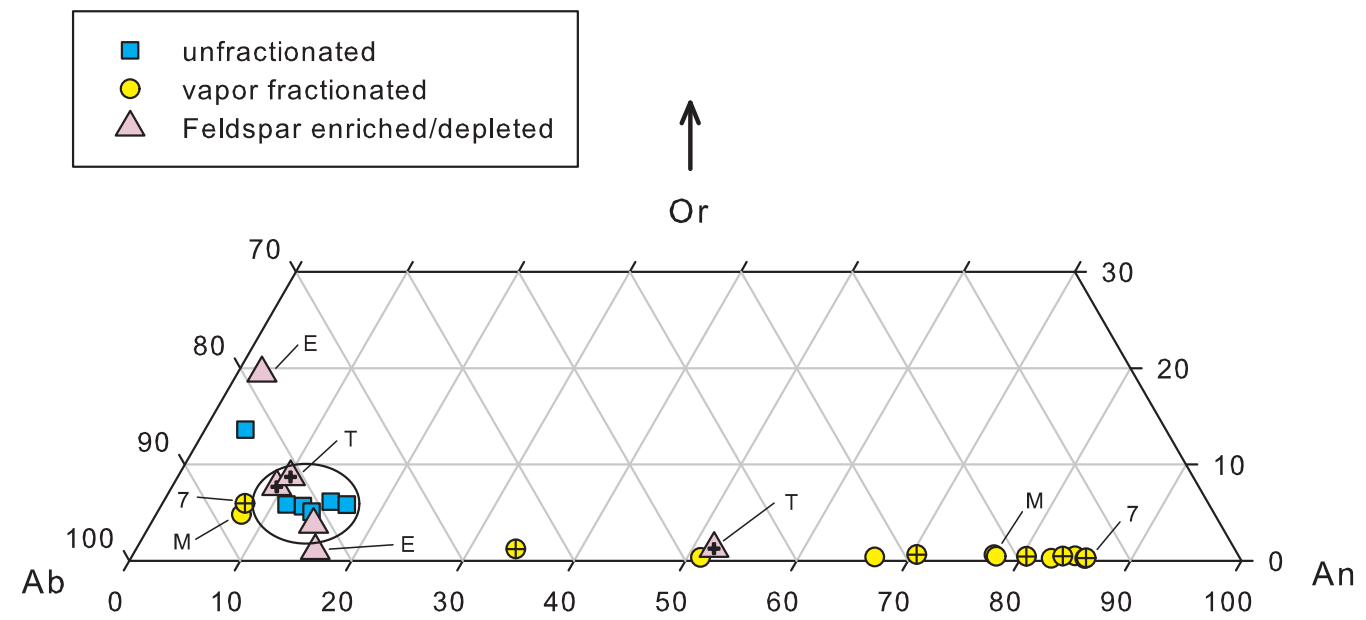

Figure 3.3: Average feldspar phase composition (mol \%) for all inclusions. Ab, Or, An stand for albite, orthoclase, and anorthite, respectively. Crossed symbols are drop-formed inclusions. Four inclusions with two feldspar phases are indicated: E is Etr-I1, T is Tdk-I1, $\mathrm{M}$ is $\mathrm{McK}-1$, and 7 is 7872-I1. Ellipse shows average $\mathrm{O}$ chondrite composition, from Brearley and Jones (1998). See section 4.3.3 for further discussion.

Table 3.4: Average phase compositions - olivine and low-Ca pyroxene, given in normalized mol \%. Fa, Wo, Fs, and En stand for fayalite, wollastonite, ferrosilite, and enstatite, respectively. Nd stands for "not detected."

\begin{tabular}{lcccc}
\hline \multirow{2}{*}{ Inclusion } & Olivine & \multicolumn{3}{c}{ Low-Ca Pyroxene } \\
& Fa & Wo & Fs & En \\
\hline $8231-I 1$ & $21.0 \pm 0.2$ & $2.7 \pm 0.6$ & $17.6 \pm 0.4$ & $79.6 \pm 0.8$ \\
Tdk-I1 & $18.4 \pm 0.4$ & $4.1 \pm 1.0$ & $15.7 \pm 1.1$ & $80.2 \pm 1.1$
\end{tabular}

continued on next page 
Table 3.4 con't

\begin{tabular}{|c|c|c|c|c|}
\hline \multirow{2}{*}{ Inclusion } & \multirow{2}{*}{$\begin{array}{c}\text { Olivine } \\
\mathrm{Fa}\end{array}$} & \multicolumn{3}{|c|}{ Low-Ca Pyroxene } \\
\hline & & Wo & Fs & En \\
\hline Tdk-I2 & $18.6 \pm 0.3$ & $2.2 \pm 1.2$ & $16.3 \pm 1.2$ & $81.5 \pm 2.2$ \\
\hline 7873-I1 & $13.5 \pm 4.5$ & nd & nd & nd \\
\hline Etr-I1 & $25.5 \pm 0.3$ & $1.5 \pm 0.1$ & $21.5 \pm 0.2$ & $77.0 \pm 0.3$ \\
\hline Glk-I1 & $11.5 \pm 2.3$ & nd & nd & nd \\
\hline Khr-I1 & $19.2 \pm 2.0$ & nd & nd & nd \\
\hline 7872-I1 & $23.6 \pm 0.3$ & nd & nd & nd \\
\hline 7869-I1 & $19.3 \pm 4.7$ & $3.2 \pm 1.1$ & $18.7 \pm 6.4$ & $78.1 \pm 6.2$ \\
\hline 7869-I2 & $23.5 \pm 3.3$ & $1.2 \pm 0.6$ & $15.9 \pm 2.0$ & $82.9 \pm 2.6$ \\
\hline 869-I1 & $16.4 \pm 6.1$ & $2.3 \pm 1.2$ & $23.7 \pm 3.3$ & $74.0 \pm 3.4$ \\
\hline 8141-I1 & $24.5 \pm 0.3$ & $1.4 \pm 0.2$ & $20.6 \pm 0.8$ & $78.0 \pm 0.9$ \\
\hline Cyn-I1 & $26.9 \pm 0.3$ & $0.7 \pm 0.2$ & $16.9 \pm 7.7$ & $82.4 \pm 7.8$ \\
\hline $\mathrm{KrC}-\mathrm{I1}$ & $22.0 \pm 0.5$ & $4.4 \pm 1.7$ & $18.1 \pm 0.5$ & $77.5 \pm 1.3$ \\
\hline McK-I1 & $25.8 \pm 0.2$ & nd & nd & nd \\
\hline McK-I2 & $24.5 \pm 0.6$ & $2.6 \pm 0.6$ & $18.1 \pm 2.2$ & $79.3 \pm 2.7$ \\
\hline 7870-I1 & $25.1 \pm 0.4$ & $1.9 \pm 0.6$ & $21.5 \pm 0.7$ & $76.6 \pm 0.7$ \\
\hline 7871-I1 & $25.6 \pm 0.3$ & nd & nd & nd \\
\hline PVM-I1 & $25.5 \pm 0.4$ & $1.9 \pm 1$ & $20.9 \pm 1.3$ & $77.2 \pm 0.8$ \\
\hline Rgl-I1 & $22.0 \pm 7.2$ & nd & nd & nd \\
\hline Par-I1 & $21.9 \pm 2$ & $8.0 \pm 2.5$ & $18.1 \pm 2.3$ & $73.9 \pm 4.4$ \\
\hline Par-I2 & $24.5 \pm 3.4$ & $0.9 \pm 0.6$ & $18.4 \pm 3.9$ & $80.7 \pm 4.4$ \\
\hline Par-I3 & $29.5 \pm 0.3$ & $3.8 \pm 0.6$ & $25.8 \pm 0.2$ & $70.4 \pm 0.6$ \\
\hline Lut-I1 & $19.9 \pm 6.3$ & $3.1 \pm 1.9$ & $10.9 \pm 3.2$ & $86.0 \pm 4.9$ \\
\hline Rfd-I1 & $18.3 \pm 3.2$ & $2.1 \pm 0.5$ & $11.1 \pm 1.2$ & $86.8 \pm 1.6$ \\
\hline 4859-I4 & $28.4 \pm 0.1$ & $1.7 \pm 0.3$ & $19.2 \pm 0.3$ & $79 \pm 0.4$ \\
\hline 4859-I18 & $30.0 \pm 0.1$ & $1.7 \pm 0.4$ & $24.6 \pm 0.2$ & $73.6 \pm 0.3$ \\
\hline 8232-I1 & $29.3 \pm 0.2$ & $1.6 \pm 0.2$ & $24.2 \pm 0.2$ & $74.2 \pm 0.3$ \\
\hline
\end{tabular}

continued on next page 
Table 3.4 con't

\begin{tabular}{lcccc}
\hline \multirow{2}{*}{ Inclusion } & Olivine & \multicolumn{3}{c}{ Low-Ca Pyroxene } \\
& $\mathrm{Fa}$ & Wo & Fs & En \\
\hline Obl-I1 & $28.8 \pm 0.6$ & $1.2 \pm 0.1$ & $23.5 \pm 0.4$ & $75.3 \pm 0.4$ \\
\hline
\end{tabular}

Table 3.5: Average phase composition - clinopyroxene, given in normalized mol \%. Wo, Fs, and En stand for wollastonite, ferrosilite, and enstatite, respectively. $\mathrm{Nd}=$ not detected.

\begin{tabular}{|c|c|c|c|}
\hline \multirow[t]{2}{*}{ Inclusion } & \multicolumn{3}{|c|}{ Clinopyroxene } \\
\hline & Wo & Fs & En \\
\hline 8231-I1 & $38.1 \pm 1.7$ & $11.2 \pm 1.3$ & $50.7 \pm 0.8$ \\
\hline Tdk-I1 & $48.8 \pm 0.6$ & $5.6 \pm 0.6$ & $45.6 \pm 0.2$ \\
\hline Tdk-I2 & $37.1 \pm 5.9$ & $8.7 \pm 2.3$ & $54.2 \pm 3.9$ \\
\hline 7873-I1 & nd & nd & nd \\
\hline Etr-I1 & $44.3 \pm 0.4$ & $8.7 \pm 0.5$ & $46.9 \pm 0.3$ \\
\hline Glk-I1 & nd & nd & nd \\
\hline Khr-I1 & $12.5 \pm 1.7$ & $21.1 \pm 1.3$ & $66.4 \pm 1.6$ \\
\hline 7872-I1 & $38.7 \pm 6.6$ & $11.0 \pm 2.1$ & $50.4 \pm 4.7$ \\
\hline 7869-I1 & $50.6 \pm 0.7$ & $1.0 \pm 0.3$ & $48.4 \pm 0.5$ \\
\hline 7869-I2 & $30.2 \pm 2.1$ & $16.3 \pm 1$ & $53.5 \pm 1.8$ \\
\hline 869-I1 & nd & nd & nd \\
\hline 8141-I1 & $45.5 \pm 0.6$ & $7.5 \pm 0.3$ & $47.0 \pm 0.8$ \\
\hline Cyn-I1 & $38.7 \pm 2.4$ & $8.8 \pm 0.8$ & $52.5 \pm 2.1$ \\
\hline KrC-I1 & $45.6 \pm 2.5$ & $6.4 \pm 0.7$ & $48.0 \pm 1.9$ \\
\hline \multirow[t]{2}{*}{ McK-I1 } & $13.1 \pm 3.3$ & $20.9 \pm 2.3$ & $66.0 \pm 3.0$ \\
\hline & $27.7 \pm 4.3$ & $15.1 \pm 1.9$ & $57.1 \pm 2.9$ \\
\hline McK-I2 & $18.9 \pm 3.0$ & $16.6 \pm 1.1$ & $64.5 \pm 2.4$ \\
\hline 7870-I1 & $44.3 \pm 2.0$ & $8.1 \pm 0.6$ & $47.7 \pm 1.4$ \\
\hline 7871-I1 & $46.1 \pm 1.3$ & $8.0 \pm 0.5$ & $45.9 \pm 1.7$ \\
\hline PVM-I1 & $45.1 \pm 0.9$ & $7.6 \pm 0.3$ & $47.3 \pm 0.6$ \\
\hline
\end{tabular}


Table 3.5 con't

\begin{tabular}{lccc}
\hline Inclusion & \multicolumn{3}{c}{ Clinopyroxene } \\
& Wo & Fs & En \\
\hline Rgl-I1 & nd & nd & nd \\
Par-I1 & $35.8 \pm 2.2$ & $11.6 \pm 0.7$ & $52.7 \pm 1.5$ \\
Par-I2 & $27.0 \pm 3.6$ & $34.9 \pm 4.9$ & $38.1 \pm 7$ \\
Par-I3 & $12.7 \pm 0.5$ & $23.2 \pm 0.2$ & $64.0 \pm 0.5$ \\
Lut-I1 & $23.7 \pm 11.7$ & $10.1 \pm 2.9$ & $66.3 \pm 11.1$ \\
Rfd-I1 & $33.7 \pm 5.6$ & $10.4 \pm 1.3$ & $56.0 \pm 4.4$ \\
4859-I4 & $41.7 \pm 0.3$ & $7.5 \pm 0.2$ & $50.8 \pm 0.3$ \\
4859-I18 & $47.3 \pm 0.8$ & $8.6 \pm 0.5$ & $44.2 \pm 0.9$ \\
8232-I1 & $45.1 \pm 0.4$ & $9.0 \pm 0.4$ & $45.9 \pm 0.1$ \\
Obl-I1 & $45.5 \pm 0.3$ & $8.2 \pm 0.2$ & $46.3 \pm 0.2$ \\
\hline
\end{tabular}

Table 3.6: Average phase compositions - feldspar, given in normalized mol \%. Ab, Or, and An stand for albite, orthoclase, and anorthite, respectively.

\begin{tabular}{lccc}
\hline Inclusion & \multicolumn{3}{c}{ Feldspar } \\
& $\mathrm{Ab}$ & $\mathrm{Or}$ & $\mathrm{An}$ \\
\hline $8231-\mathrm{I} 1$ & $82.7 \pm 1.6$ & $13.6 \pm 1.9$ & $3.7 \pm 1.9$ \\
Tdk-I1 & $81.2 \pm 2.0$ & $8.7 \pm 3.8$ & $10.2 \pm 3.4$ \\
& $46.8 \pm 12.3$ & $1.3 \pm 0.5$ & $51.9 \pm 12.7$ \\
Tdk-I2 & $17.0 \pm 2.3$ & $0.2 \pm 0.1$ & $82.7 \pm 2.3$ \\
$7873-\mathrm{I} 1$ & $\mathrm{nd}$ & $\mathrm{nd}$ & $\mathrm{nd}$ \\
Etr-I1 & $82.7 \pm 2.5$ & $1.0 \pm 0.1$ & $16.2 \pm 2.5$ \\
& $78.3 \pm 1.2$ & $19.5 \pm 2.8$ & $2.2 \pm 1.7$ \\
Glk-I1 & $\mathrm{nd}$ & $\mathrm{nd}$ & $\mathrm{nd}$ \\
Khr-I1 & $22.0 \pm 6.8$ & $0.6 \pm 0.5$ & $77.4 \pm 6.9$ \\
$7872-\mathrm{I} 1$ & $13.9 \pm 4.2$ & $0.3 \pm 0.2$ & $85.9 \pm 4.3$ \\
& $86.6 \pm 1.8$ & $6.0 \pm 1.5$ & $7.4 \pm 0.7$ \\
$7869-\mathrm{I} 1$ & $28.9 \pm 3.5$ & $0.6 \pm 0.2$ & $70.5 \pm 3.5$ \\
continued & & &
\end{tabular}


Table 3.6 con't

\begin{tabular}{|c|c|c|c|}
\hline \multirow[t]{2}{*}{ Inclusion } & \multicolumn{3}{|c|}{ Feldspar } \\
\hline & $\mathrm{Ab}$ & Or & An \\
\hline 7869-I2 & $48.5 \pm 1.8$ & $0.3 \pm 0.1$ & $51.2 \pm 1.8$ \\
\hline 869-I1 & nd & nd & nd \\
\hline 8141-I1 & $81.6 \pm 5.1$ & $5.7 \pm 1.2$ & $12.8 \pm 4.5$ \\
\hline Cyn-I1 & $32.8 \pm 1.9$ & $0.4 \pm 0.1$ & $66.8 \pm 1.9$ \\
\hline KrC-I1 & $64.6 \pm 8.3$ & $1.2 \pm 0.7$ & $34.1 \pm 8.8$ \\
\hline \multirow[t]{2}{*}{ McK-I1 } & $21.8 \pm 2.8$ & $0.4 \pm 0.2$ & $77.7 \pm 2.9$ \\
\hline & $87.5 \pm 1.8$ & $4.8 \pm 0.8$ & $7.7 \pm 2.0$ \\
\hline McK-I2 & $14.7 \pm 1.0$ & $0.5 \pm 0.4$ & $84.8 \pm 1.3$ \\
\hline 7870-I1 & $82.9 \pm 0.7$ & $7.7 \pm 1.0$ & $9.4 \pm 1.2$ \\
\hline 7871-I1 & $78.8 \pm 3.9$ & $6.1 \pm 0.9$ & $15.1 \pm 3.3$ \\
\hline PVM-I1 & $82.9 \pm 1.9$ & $5.9 \pm 2.1$ & $11.2 \pm 0.5$ \\
\hline Rgl-I1 & nd & nd & nd \\
\hline Par-I1 & $14.0 \pm 2.2$ & $0.2 \pm 0.1$ & $85.8 \pm 2.1$ \\
\hline Par-I2 & nd & nd & nd \\
\hline Par-I3 & nd & nd & nd \\
\hline Lut-I1 & $19.1 \pm 6.2$ & $0.4 \pm 1.1$ & $80.4 \pm 5.9$ \\
\hline Rfd-I1 & nd & nd & nd \\
\hline 4859-I4 & $81.6 \pm 1.5$ & $3.7 \pm 0.7$ & $14.7 \pm 2.0$ \\
\hline 4859-I18 & $77.5 \pm 5.2$ & $5.8 \pm 1.3$ & $16.7 \pm 6.1$ \\
\hline 8232-I1 & $81.1 \pm 1.5$ & $5.1 \pm 0.7$ & $13.8 \pm 1.9$ \\
\hline Obl-I1 & $82.3 \pm 2.1$ & $4 \pm 1.1$ & $13.7 \pm 1.9$ \\
\hline
\end{tabular}




\section{Large inclusions in ordinary chondrites}

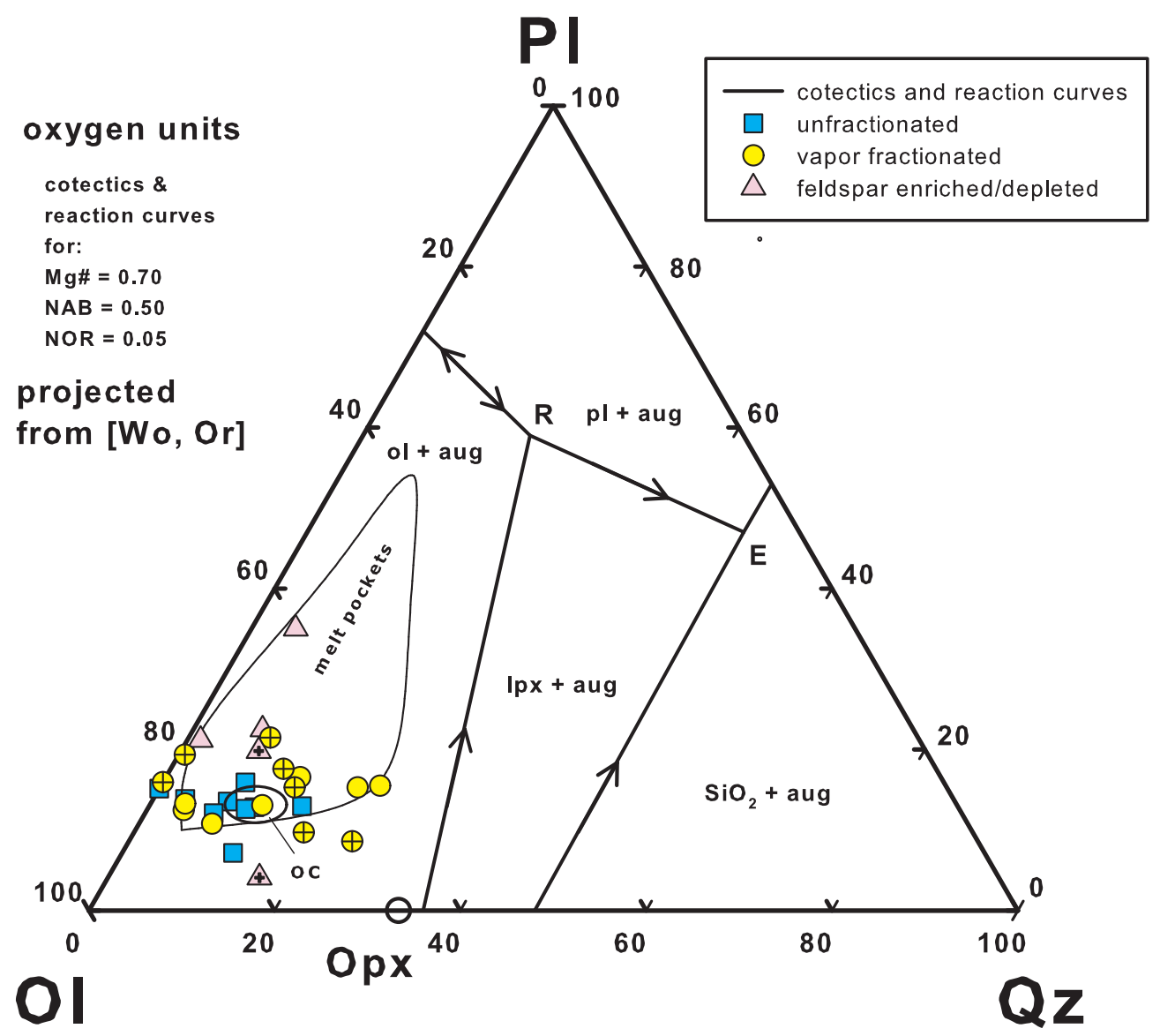

Figure 3.4: All inclusions on a normative olivine (Ol)- plagioclase (Pl)- quartz (Qz) ternary liquidus diagram, with cotectics and reaction curves shown. Figure adapted from Ruzicka et al. (2012a). Average O chondrite (OC) from Jarosewich (1990). Symbols used are: aug = augite, $\mathrm{lpx}=$ low-Ca pyroxene, $\mathrm{SiO}_{2}=$ silica, $\mathrm{NAB}=$ normative albite, $\mathrm{NOR}=$ normative orthoclase, $\mathrm{E}=$ eutectic, $\mathrm{R}=$ olivine-pyroxene-feldspar peritectic. Crossed symbols are those inclusions that were droplet formed; calculated error is approximately represented by symbol size. 


\section{Discussion}

The data collected here allow for some preliminary interpretations. Table 3.1 lists the inclusions, as well as their host meteorite type and whether or not their olivine and low-Ca pyroxene is equilibrated. Ten inclusions are from hosts with a higher metamorphic grade (types 5-6; olivine and low-Ca pyroxene are equilibrated), four are contained in breccias, which contain clasts of at least two different types, and fifteen are in weakly metamorphosed (types 3-4; olivine and low-Ca pyroxene are unequilibrated) hosts. (Inclusions are distinguished in breccias by their igneous texture; the host material has a chondritic texture metamorphosed to different degrees.)

There is no correlation between host type and inclusion equilibration; there are inclusions that were equilibrated before becoming incorporated into their host (8141I1, Par-I3, possibly 7870-I1), as well as hosts that were equilibrated before an inclusion was added (Tdk-I1, Tdk-I2, and 7873-I1). This indicates that material in the early solar system was mixing over long timescales as metamorphism was ongoing, and provides insight into the timing of inclusion melting relative to host metamorphism.

Table 3.1 also indicates whether the $\mathrm{FeO}$ content of the inclusion's olivine and low-Ca pyroxene is similar to average host: 13 inclusions are similar, while 16 are not. Of those that differ in composition, only one, Etr-I1, is higher in FeO than its host. The olivine and low-Ca pyroxene of the remaining 15 are lower in $\mathrm{FeO}$ than their host. There is no correlation between this parameter and any other property of the inclusions, though five of the inclusions with similar $\mathrm{FeO}$ content to their hosts7871-I1, PVM-I1, 4859-I4, 4859-I18, and 8232-I1- are equilibrated inclusions in hightype hosts. These inclusions may have therefore acquired the phase chemistry of their 
hosts subsequent to becoming mixed in (see section 4.3.1 for further discussion).

Figure 3.4 shows calculated normative compositions projected onto an olivineplagioclase-quartz ternary diagram. All of the inclusions in this study plot close to the average $\mathrm{O}$ chondrite composition. None of the inclusions plots near any of the cotectics or reaction curves, which would be expected for material that experienced igneous processing. This implies that none of the inclusions in this study derive from a differentiated source.

Table 3.2 lists further physical properties of the inclusions, including texture and grain size. Observed trends are discussed in more detail below.

\subsection{Drop-formed inclusions}

Nine inclusions (Tdk-I1, 7869-I1, 7872-I1, 7870-I1, KrC-I1, Lut-I1, Rfd-I1, Par-I2, and Par-I3) have compelling evidence that they, like chondrules, crystallized as freefloating droplets in space. These inclusions are round in shape, have textures commonly seen in chondrules, concentric textures, radial variations in texture and/or chemistry, and appear to have interacted with their surroundings (figure 4.1). Many of them have a texturally distinct rim or mantle, also a common feature of chondrules. Rfd-I1 has possibly the most compelling evidence for having interacted with its surroundings. Its outer $\sim 100 \mu \mathrm{m}$ is chemically distinct from the interior, which is otherwise relatively equilibrated. Olivine grains in the altered outer region are distinctly more fayalitic, and feldspar is more sodic, suggesting metasomatic addition of Fe and Na.

Interestingly, all nine of these inclusions may have been affected by a volatility fractionation process. The evidence for five- 7869-I1, 7872-I1, KrC-I1, Rdf-I1, and 
Lut-I1- is fairly clear: for these, volatile elements are depleted, and more volatile elements (as measured by 50\% condensation temperature) are more depleted (see figure 4.2).

For three of the droplet inclusions (Par-I2, Par-I3, 7870-I1), the evidence for a volatility-based fractionation is less obvious. Par-I2 has a largely chondritic (i.e., not vapor-fractionated, see figure A.43) bulk chemistry, but it is depleted in both the most volatile and most refractory elements, and enriched in those elements of moderate volatility. This suggests that this inclusion did undergo fractional condensation; condensates must have been removed from the system as cooling continued. This would allow for both the most refractory and most volatile elements to have been excluded from the final composition. Similarly, Par-I3 is also nearly chondritic, but is strongly depleted in $\mathrm{Na}$ (see figure A.45), which is difficult to explain without a vapor process.

Tdk-I1 has a similar pattern to Par-I2; it is depleted in Al, Na, and $\mathrm{K}$, relative to the elements of intermediate volatility. Thus it, too, may be a result of fractional condensation, though this inclusion may also be interpreted as being depleted in a feldspathic component (see section 4.3.2).

7870-I1 may be an exception; it is clearly droplet-formed but does not appear to have been volatility-fractionated. It has a generally chondritic bulk chemistry, with enrichments in both the most refractory $(\mathrm{Al}, \mathrm{Ti})$ and most volatile $(\mathrm{Na}, \mathrm{K})$ elements. The enrichment of $\mathrm{Al}$ and $\mathrm{Ti}$ allow for the interpretation that this inclusion was vapor fractionated, with later metasomatism causing the observed enrichment of $\mathrm{Na}$ and K. However, this can also be interpreted as an enrichment in feldspar, which is consistent with its modal abundance (see table 3.3) and normative plagioclase (see 
figure 3.4). Additionally, the EDS element maps do not indicate an enrichment of alkalis at the edges, which weakens the argument that the $\mathrm{Na}$ and $\mathrm{K}$ enrichment is due to metasomatism. $7870-\mathrm{I} 1$ is therefore probably a feldspar-enriched droplet (see section 4.3.2).

In general, there is a trend for droplets to be refractory enriched and volatile depleted. These inclusions, as a group, thus potentially formed as melt droplets that experienced a kinetic process, such as condensation (e.g. Tdk-I1 and Par-I2) or evaporative heating. Some, such as Lut-I1, subsequently experienced metasomatism, with an influx of alkalis, Fe, P, and Cl. Still others (7870-I1, Tdk-I1) may have formed as a a feldspar-enriched/depleted droplet, possibly by a different process (see section $4.3 .2)$ 

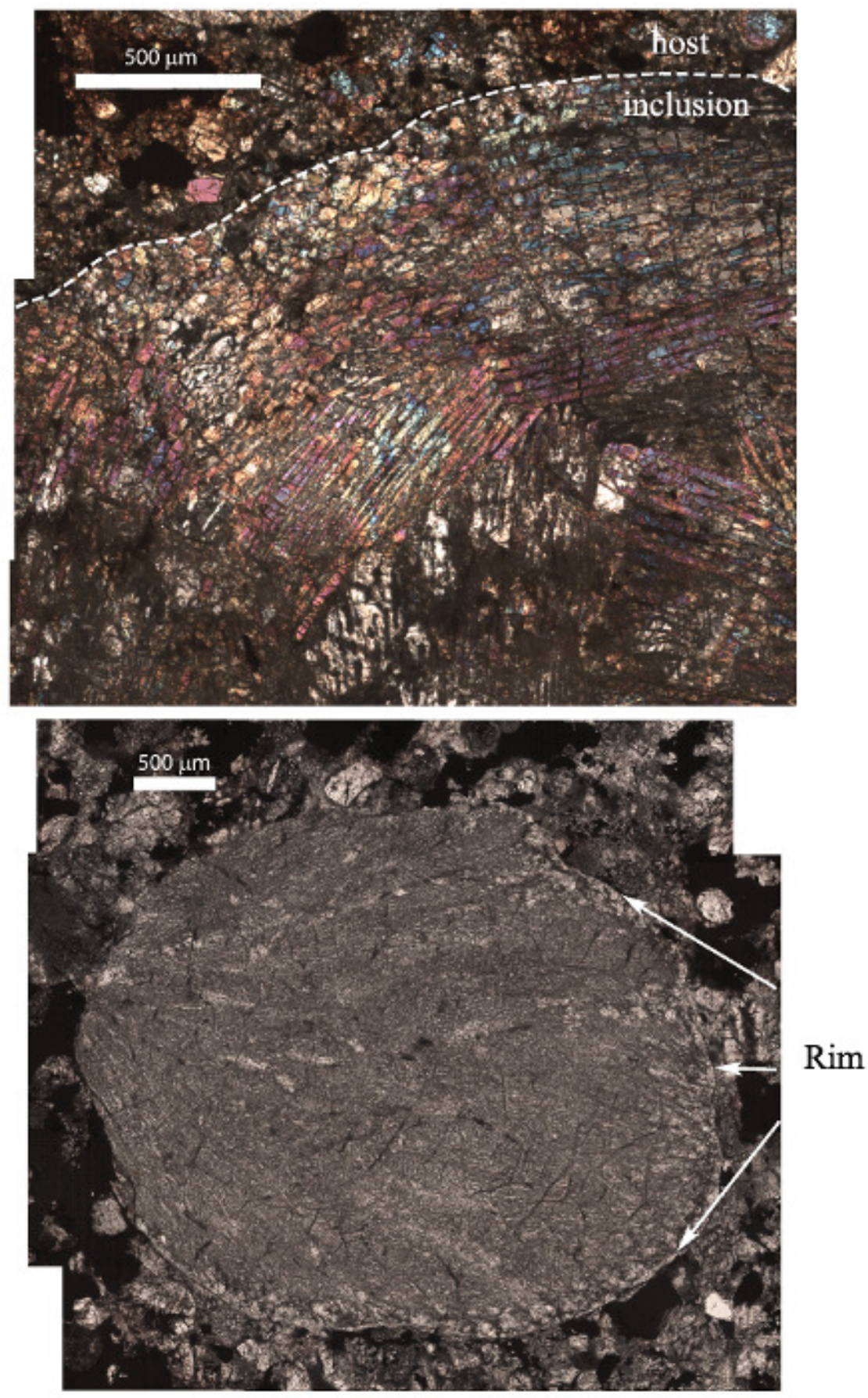

Figure 4.1: Examples of droplet-formed objects: 7870-I1 (top, under cross-polarized light) and Tdk-I1 (bottom, under plane transmitted light). Note the concentric orientation of skeletal olivine and the transition to a more porphyritic texture near the host in 7870-I1, and the clear rim surrounding most of Tdk-I1. 


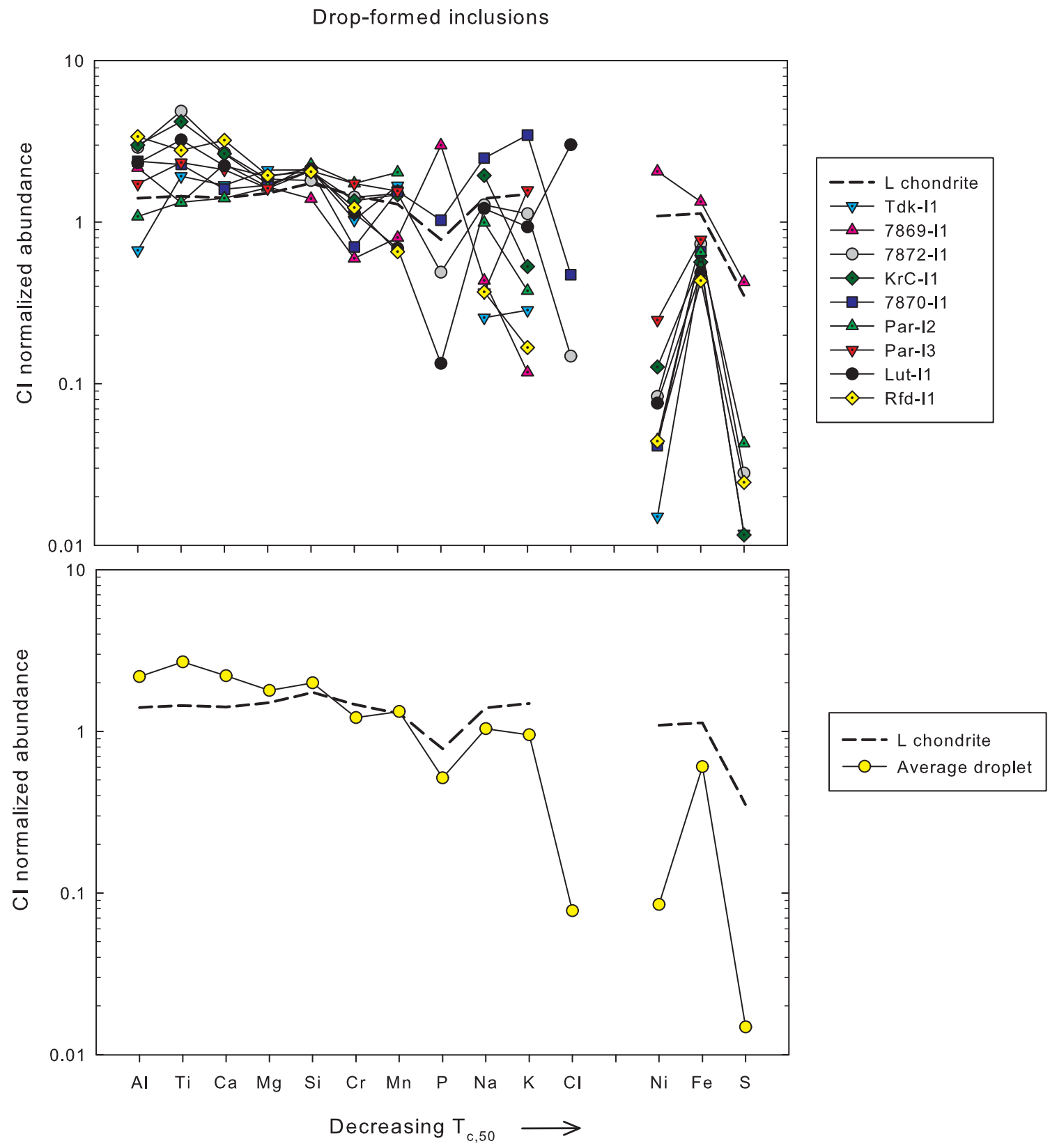

Figure 4.2: CI normalized bulk chemistry of all droplet-formed inclusions (top) and of their average composition (bottom). Elements are arranged in order of increasing volatility, defined by $50 \%$ condensation temperature $\left(\mathrm{T}_{\mathrm{c}, 50}\right)$, with lithophile elements on the left and siderophile/chalcophile elements on the right. L chondrite reference data from Jarosewich (1990). 


\subsection{Shock melt}

The data collected for this study is not sufficient to definitively identify a formation process for any inclusion. However, there is compelling evidence that at least one, and probably several, of the inclusions in this study are shock melts. 869-I1 is contained in a host meteorite, NWA 869, that is relatively well studied (e.g., Metzler et al., 2011) The CML sample studied here has numerous shock-darkened regions, and the inclusion itself appears to have intruded into and partially melted the host (see figure 4.3). The inclusion also resembles, in texture and in olivine chemistry, clasts in other samples of NWA 869 that others have identified as impact melt rocks (Metzler et al., 2011).

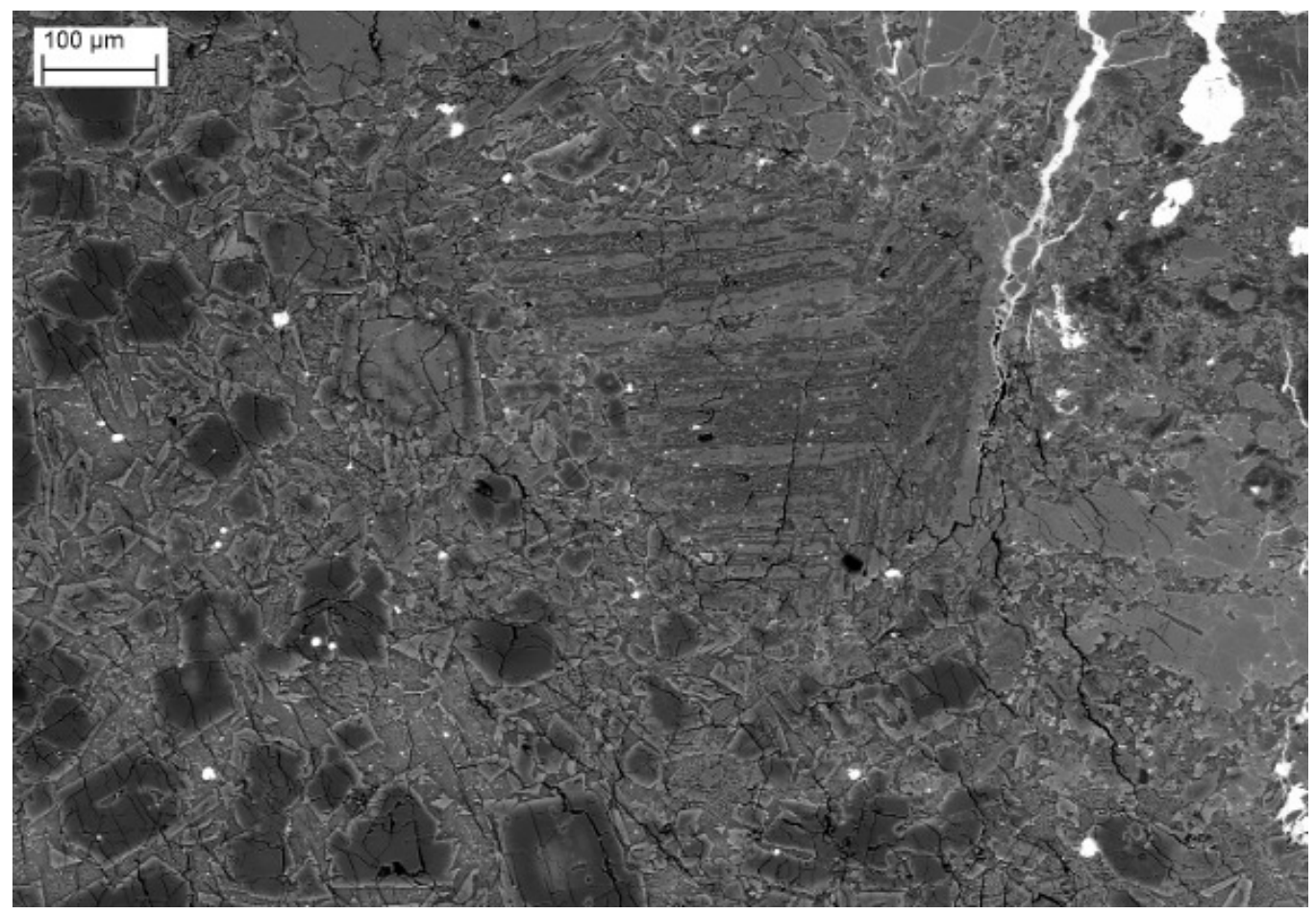

Figure 4.3: Shock-melted material of 869-I1 engulfing a chondrule as the intruding melt material partially melted the host. 


\subsubsection{K enrichment}

869-I1 is roughly chondritic in bulk chemistry, except for a striking enrichment in potassium (see figure 4.4). It has been suggested (Chen and El Goresy, 2000) that Na-K fractionation can occur at high pressures. The apparent excess of K, without an excess of $\mathrm{Na}$, suggests that metasomatism is not responsible for the K enrichment. Another inclusion, 8231-I1 is also in close proximity to shock-darkened regions, and is itself bisected by a shock vein. This inclusion is also enriched in $\mathrm{K}$ with an otherwise chondritic bulk chemistry. Further study may illuminate whether this K enrichment is a result of metasomatism, or whether it may be a result of shock processes.

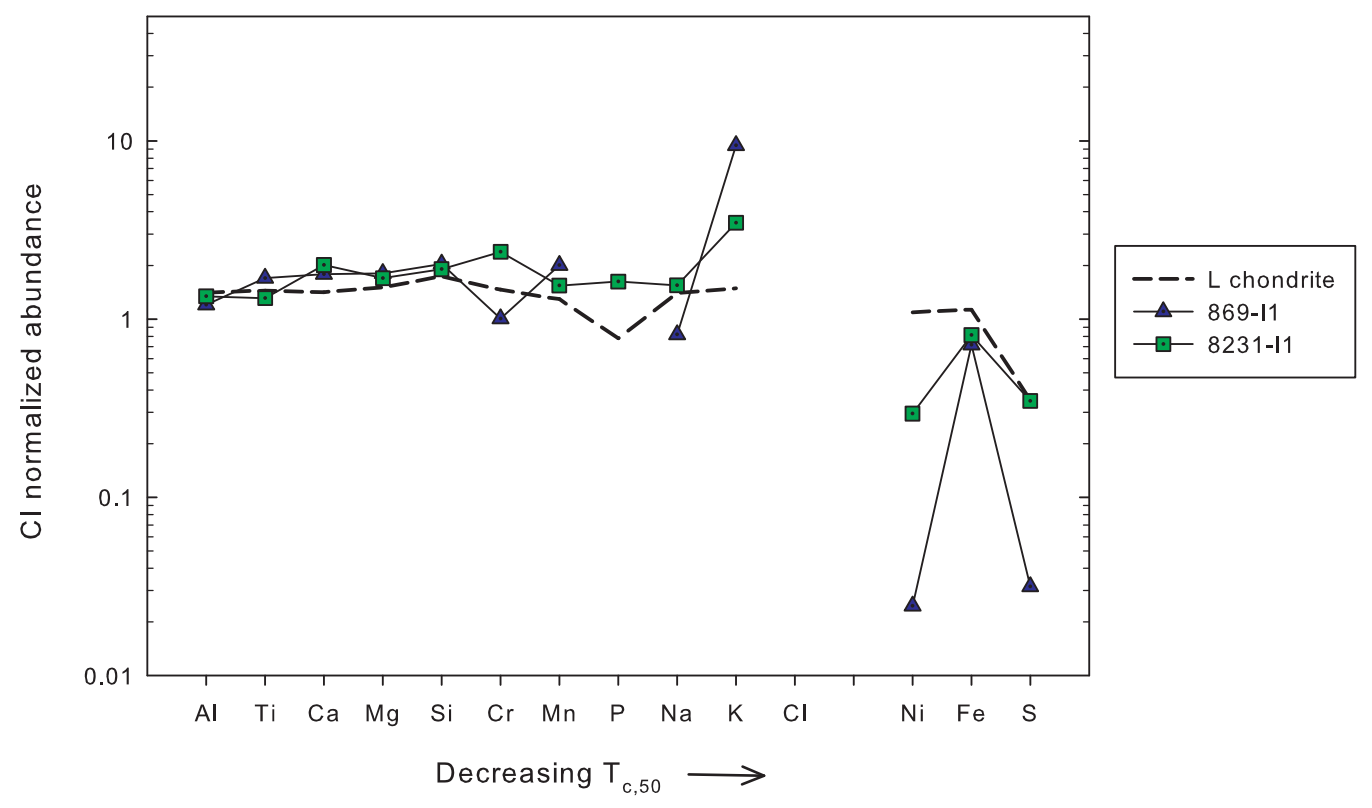

Figure 4.4: Two inclusions that are enriched in potassium, without a clear enrichment in $\mathrm{Na}$ or other elements associated with metasomatism. These inclusions are, for the most part, otherwise generally chondritic (besides the ubiquitous metal and sulfide depletion). 


\subsection{Trends in bulk chemistry}

Broadly, the lithophile-element chemistry of the inclusions can be described as either unfractionated (seventeen inclusions) or vapor fractionated (eleven inclusions, though four inclusions with an unfractionated bulk chemistry may have once been vapor fractionated, and then experienced some metasomatism). There are several variation of both themes, discussed in more detail below, but in general it appears that some inclusions have undergone vapor processes and others have not (see figure 4.5) This supports the general conclusions of Ruzicka et al. (1998, 2000), in which large igneous inclusions were subdivided into Na-rich (unfractionated) and Na-poor (vapor fractionated) groups.

Figure 4.5 shows CI-normalized ratios of $\mathrm{Na} / \mathrm{Al}$ and $\mathrm{Mg} / \mathrm{Al}$. With a few exceptions, the vapor fractionated inclusions have a notably lower $\mathrm{Na} / \mathrm{Al}$ ratio than the unfractionated inclusions; $\mathrm{Na}$ is much more volatile that $\mathrm{Al}$, and so depletion in $\mathrm{Na}$ drives the ratio down. Par-I2 is the lone vapor fractionated inclusion that has a $\mathrm{Na} / \mathrm{Al}$ ratio greater than $\sim 0.65$; this inclusion has lost both its most refractory and most volatile elements, possibly through fractional condensation, which increases that ratio. In addition, the $\mathrm{CI}$-normalized $\mathrm{Mg} / \mathrm{Al}$ ratio is less than or equal to one for the vapor fractionated group, also consistent with a volatility-controlled process, as $\mathrm{Al}$ is more refractory than $\mathrm{Mg}$.

Figure 4.6 shows CI-normalized $\mathrm{Na} / \mathrm{Mg}$ plotted against $\mathrm{Al} / \mathrm{Mg}$. Again the two groups form discernibly different trends. These two ratios correlate almost 1:1 for the unfractionated and feldspar-enriched inclusions, indicating that there has not been fractionation. 


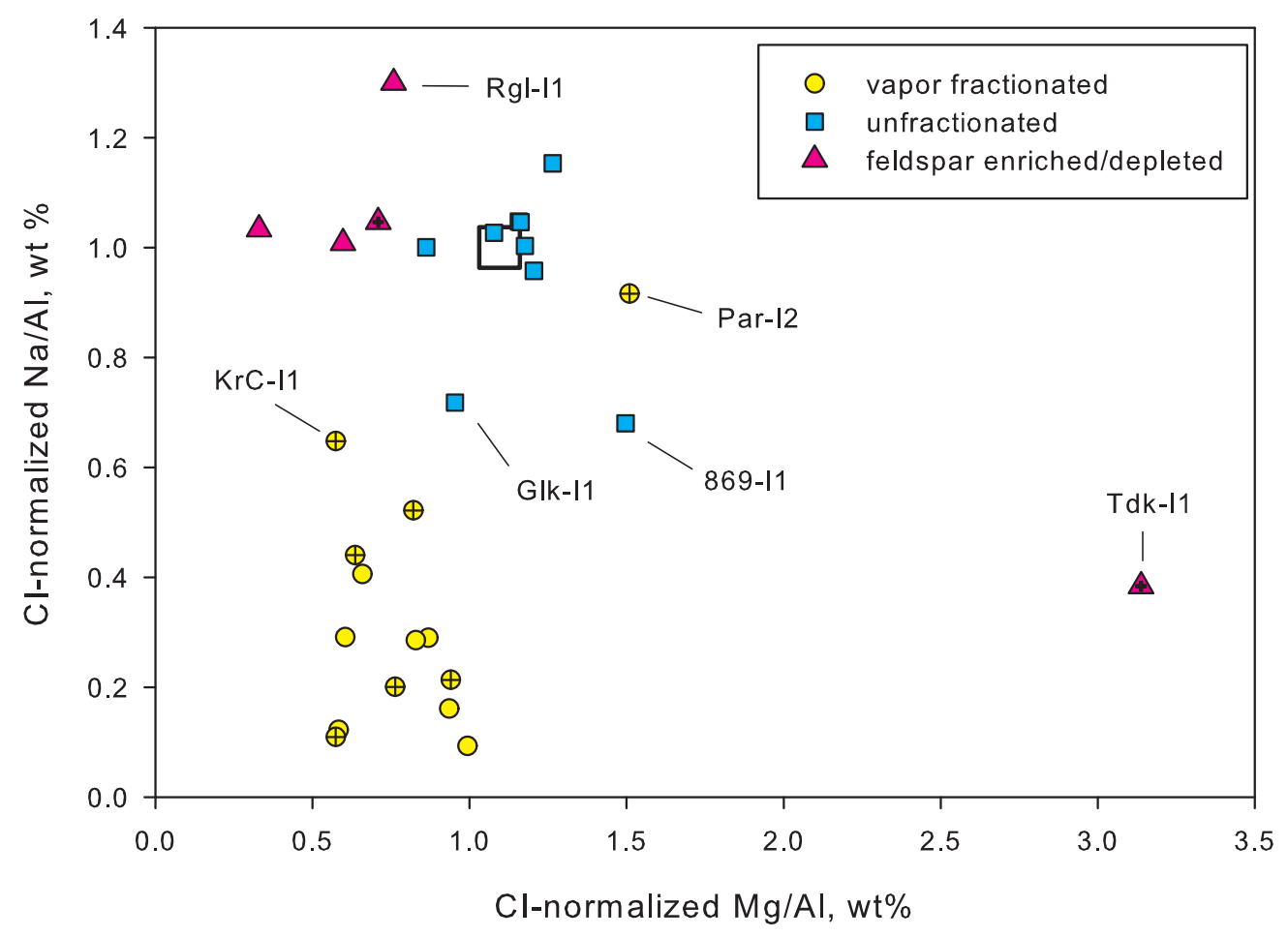

Figure 4.5: CI-normalized ratios of $\mathrm{Mg} / \mathrm{Al}$ vs $\mathrm{Na} / \mathrm{Al}$ (Wt). The vapor-fractionated inclusions have, in general, a distinctly lower $\mathrm{Na} / \mathrm{Al}$ ratio than unfractionated, as a result of the loss of more volatile Na relative to more refractory Al. Par-I2 is the only obvious outlier. It has lost both $\mathrm{Na}$ and $\mathrm{Al}$, possibly due to fractional condensation, resulting in a higher ratio. The vapor-fractionated inclusions also appear to have $\mathrm{Mg} / \mathrm{Al}$ less than or equal to chondritic. This is also consistent with some enrichment in an Al-rich component relative to $\mathrm{Mg}$. The unfractionated and feldspar-enriched inclusions have higher $\mathrm{Na} / \mathrm{Al}$ and chondritic to subchondritic $\mathrm{Mg} / \mathrm{Al}$, and so could have formed by melting of chondritic material, and (in some cases) feldspar-enrichment of such material. Glk-I1 and 869-I1 are probably shock-melted material (see section 4.2); volatile $\mathrm{Na}$ may have been vaporized. The square shows average chondrite composition (from Brearley and Jones, 1998). 


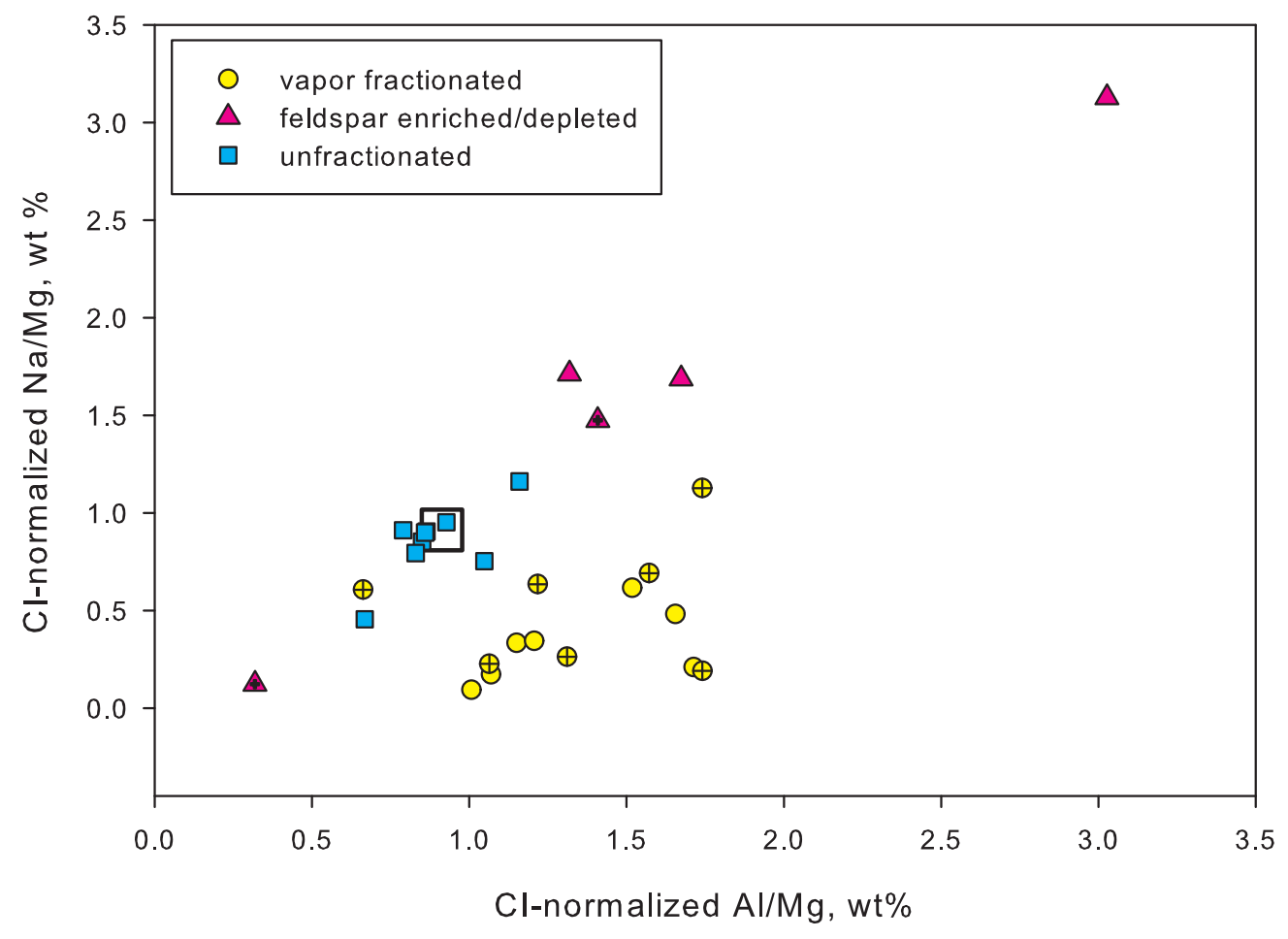

Figure 4.6: CI-normalized $\mathrm{Al} / \mathrm{Mg}$ vs $\mathrm{Na} / \mathrm{Mg}$ (Wt). The two groups again plot in distinct groups. For the chondritic, unfractionated inclusions, the ratio of refractory $\mathrm{Al} / \mathrm{Mg}$ and volatile $\mathrm{Na} / \mathrm{Mg}$ plot close to 1 , and in a general 1:1 trend. The vapor-fractionated group has a $\mathrm{Na} / \mathrm{Mg}$ ratio $\leq 1$. The feldspar-enriched inclusions, with CI-normalized $\mathrm{Al} / \mathrm{Mg}$ and $\mathrm{Na} / \mathrm{Mg}$ values $>1.2$, plot with the chondritic group, suggesting they may be melted chondrite with an excess of feldspar. The square shows average chondrite composition (from Brearley and Jones, 1998). Crossed symbols are droplet-formed inclusions.

\subsubsection{Unfractionated}

Fourteen of the inclusions have an unfractionated (essentially chondritic) bulk chemistry, except for depletions in the siderophile and chalcophile elements $(\mathrm{S}, \mathrm{Ni}, \mathrm{Fe}$; see table 3.1). Seven of these are in chondrites of high metamorphic grade (types 5-6, figure 4.7). Of these seven, six (Etr-I1, 7871-I1, PVM-I1, 4859-I4, 4859-I18, and 
8232-I1) are themselves equilibrated. These six are therefore difficult to interpret, because while their chemistry may be a primary characteristic, it is possible that they were incorporated into their host meteorite before it was metamorphosed, and that signatures of previous processing were erased during metamorphism.

Three of these objects do, however, have evidence that they were once independent objects. That is, there is evidence that their rim is a primary feature through which there was a chemical exchange with its surroundings. 4859-I4, 4859-I18, and 8232-I1 all have some sort of rim and/or a radial variation in texture or mineralogy, though their sizes and shapes do not support a drop-formed origin. Also of note, Etr-I1 and 4859-I4 both appear enriched in a feldspar component, discussed further below (see section 4.3.2).

An additional seven inclusions have an unfractionated bulk chemistry and exist in a weakly metamorphosed host (types 3-4, figure 4.8). Four (8231-I1, 8141-I1, 7870I1, and Par-I3) are themselves equilibrated, and so the inclusion material must have been thermally processed before it was mixed into the host. This processing may have erased indications of previous chemical processes.

Four inclusions- 7873-I1, Glk-I1, 869-I1, and Rgl-I1- have an unfractionated bulk chemistry and are themselves unequilibrated. For these inclusions, the chondritic compositions must be a primary feature, not established by metamorphism that affected the host. The host meteorites of these inclusions are H5-6, L3.6, L3-6, and LL3.4, respectively. The chondritic composition of 7873-I1, at least, can also thus be inferred to postdate the metamorphism of its host. These inclusions are also united by their texture; all four are olivine microporphyries with zoned, euhedral olivine phenocrysts in a glassy mesostasis. There is strong evidence that 869-I1 is shock melt 
(see section 4.2); these others may be good candidates to investigate for indications of shock.

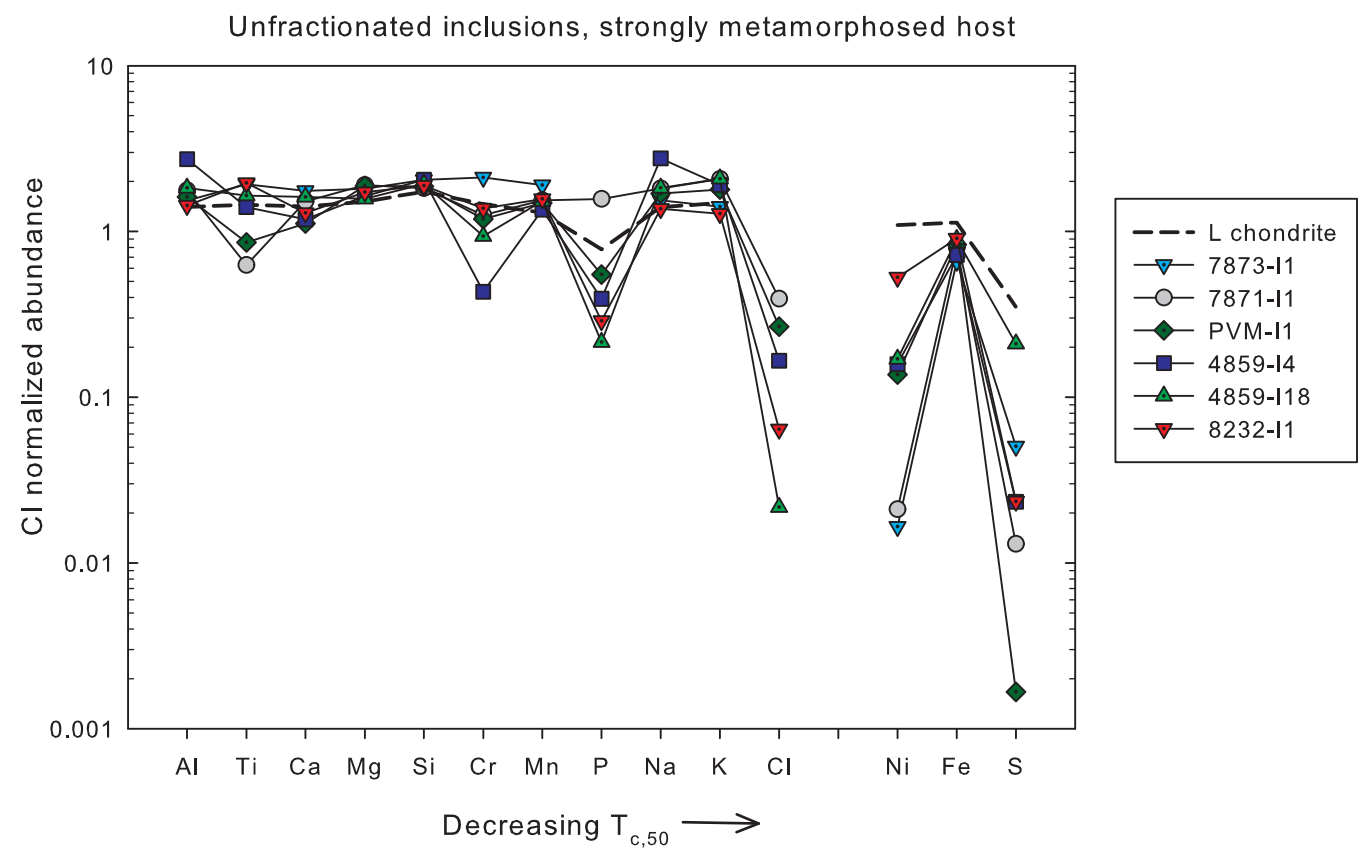

Figure 4.7: CI-normalized bulk chemistry of unfractionated inclusions in a high-type host. 


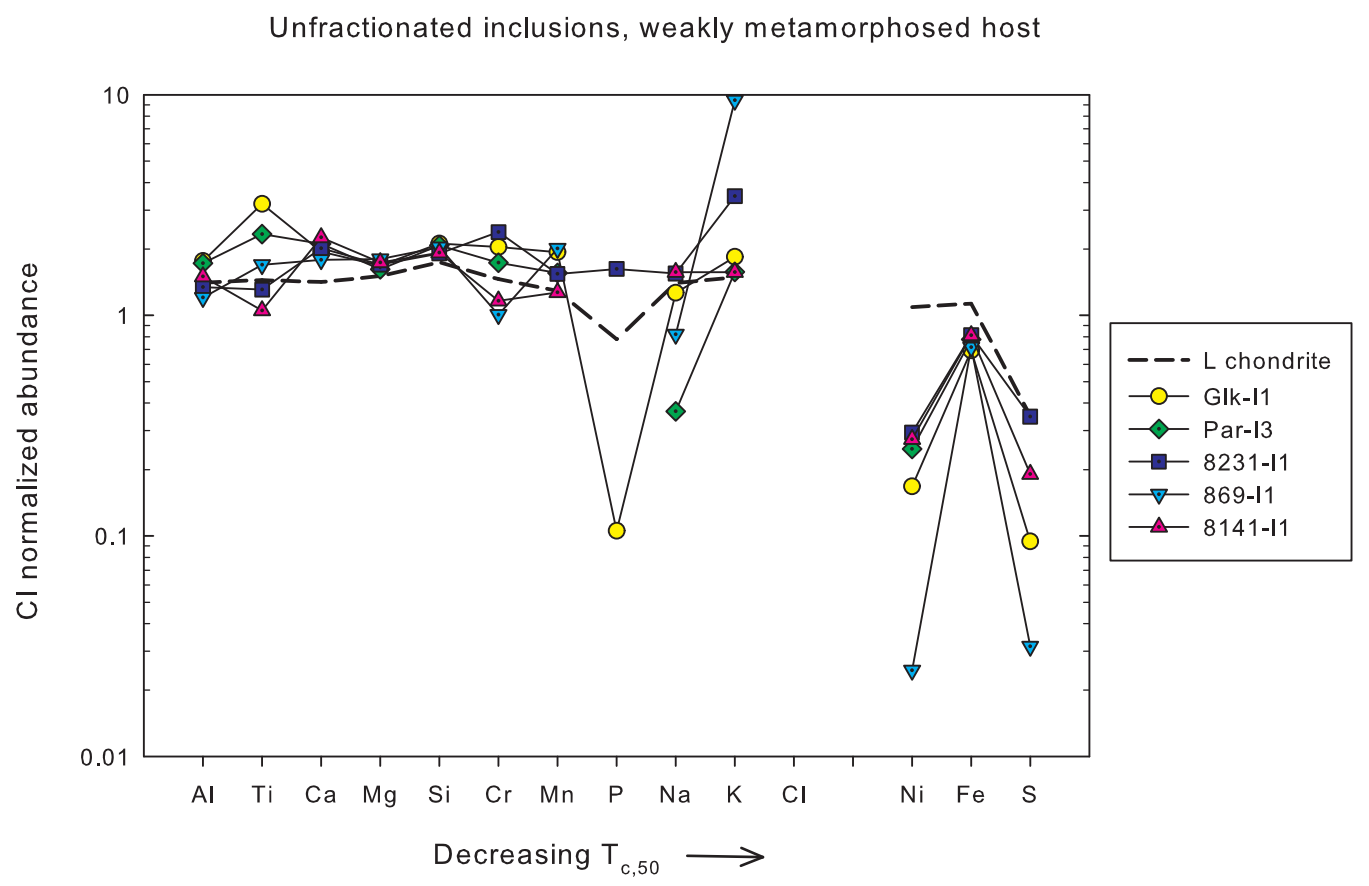

Figure 4.8

\subsubsection{Feldspar enriched}

Five inclusions- Etr-I1, 7870-I1, 4859-I4, Rgl-I1, and KrC-I1- appear enriched in a feldspathic component when their bulk chemistry is plotted on an Ol-Pl-Qz ternary. Feldspar melts at a lower temperature than olivine or pyroxene, and so these inclusiosn may have originated as a partial melt of a chondritic source.

Etr-I1 and 4859-I4 have chondritic abundances of all elements except those found in feldspar, in which they are enriched. Additionally, they both have a high modal abundance of feldspar compared to other inclusions in this study. (see table 3.3 and figure 4.9).

7870-I1 also has a high percentage of feldspar, and an enrichment in $\mathrm{Na}$ and $\mathrm{K}$ that may be a result of an enrichment of a feldspathic component. It also has 
substantial evidence for having crystallized as a droplet (see sections 4.1 and A.14). One interpretation for the history of this inclusion may be that a partial melt of a chondritic source, such as the L chondrite parent object, was splashed or jetted into space, where small droplets then crystallized.

KrC-I1 plots very close to 7870-I1 and 4859-I1 on a Ol-Pl-Qz ternary (see figure 3.4). Its bulk chemistry and modal abundance, however, do not necessarily support the conclusion that it is similarly enriched in feldspar. It appears to have been vapor fractionated (see figure A.28) and then metasomatized. An influx of sodium would account for the observed increase in normative albite. This interpretation is supported by its $\mathrm{Na} / \mathrm{Al}$ ratio (see figure 4.5 ), which is subchondritic.

Another inclusion in this study, Tdk-I1, has a similar but opposite bulk chemistry pattern; that is, it is depleted in feldspar and $\mathrm{Al}, \mathrm{Na}$, and $\mathrm{K}$, and correspondingly enriched in Mg. This object may thus represent a counterpart to the feldspathic precursor melt of 7870-I1, 4859-I4 and Etr-I1. 


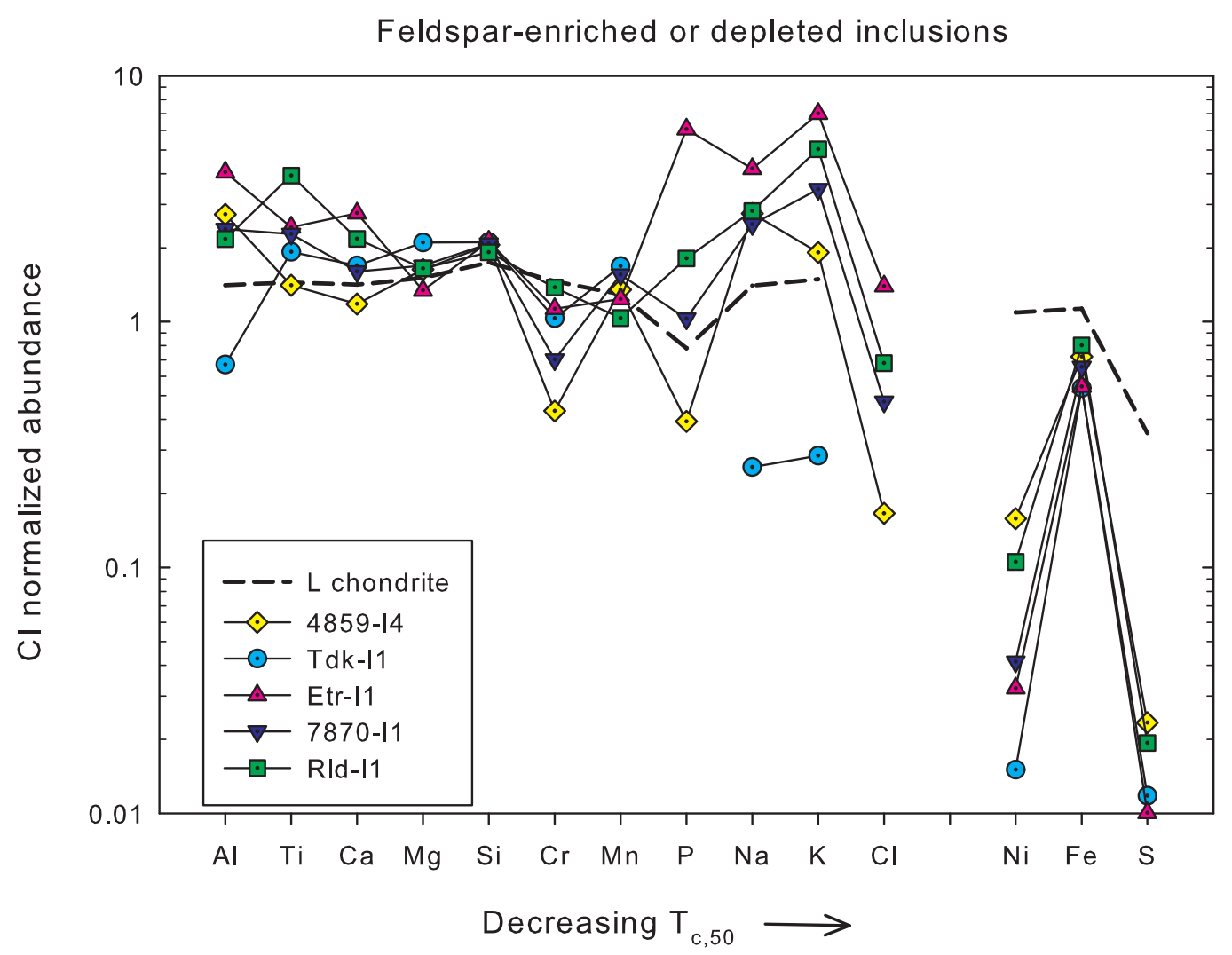

Figure 4.9: Feldspar enrichment

\subsubsection{Bimodal Feldspar}

Four inclusions- Etr-I1, Tdk-I1, 7872-I1, and Mck-I1- have two distinct feldspar phases (see figure 3.3). The majority of a Ca-depleted feldspar phase of Etr-I1 is in close proximity to a large phosphate grain (see figure A.9), suggesting that $\mathrm{Ca}$ in the primary feldspar had migrated into the phosphate as it grew.

The reason for the existence of two feldspar phases in the other three inclusions is less clear. It is possible that the two phases crystallized in succession during primary crystallization, reflecting a change in composition of the melt. Or, subsequent meta- 
somatism could have altered some of the primary feldspar, resulting in two phases.

\subsubsection{Volatility trends}

Nine of the inclusions investigated show a depletion of more volatile elements, with a clear trend of greater depletion with higher volatility (see figure 4.10). Three inclusions in the study showed an enrichment in the most refractory elements (see figure 4.11). An additional two appear to be most enriched in moderately volatile elements. Eleven of the volatility-fractionated inclusions also have evidence for forming as a free-floating droplet, but five inclusions (7869-I2, Cyn-I1, McK-I1, McK-I2, and ParI1) do not. Par-I1 does appear to have a rim, and so may have been an independent object, but the other four appear to be fragments of larger igneous objects. This could mean that the source material was volatility fractionated before melting, or that the melt itself experienced a vapor process. 
Vapor-fractionated inclusions, volatile-depleted

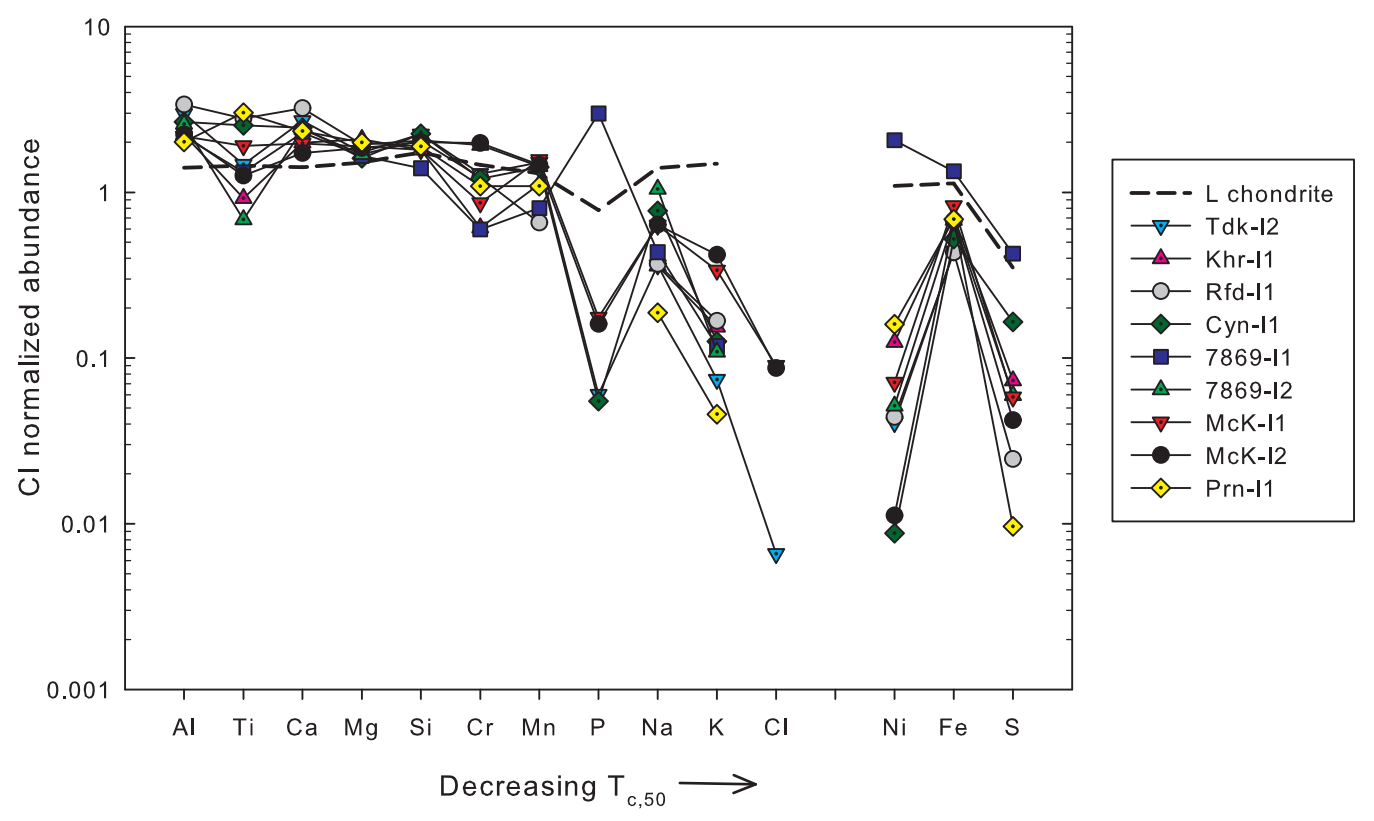

Figure 4.10: Ten inclusions showing a general trend of volatile depletion in their bulk chemistry, compared to a reference chondrite (L). Overall, a smooth trend of greater depletion with greater volatility can be seen. Phosphorous is a notable exception. Phosphates were not present in many of the inclusions studied, and when they were it was usually as a very minor phase. A few notable exceptions, such as 7869-I1 here, contained larger amounts of phosphates. 


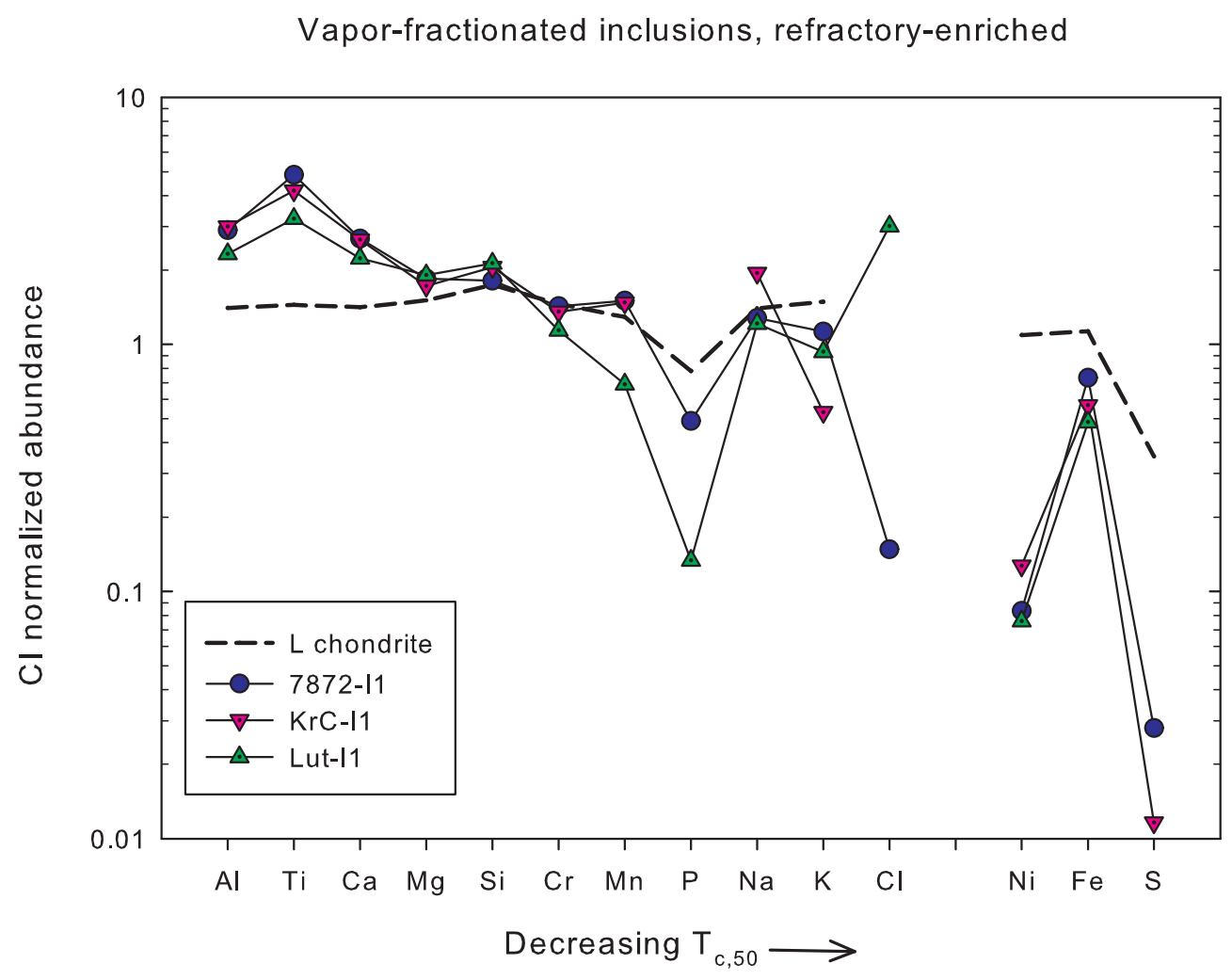

Figure 4.11: Four inclusions showing a general trend of refractory enrichment in their bulk chemistry, compared to a reference chondrite (L).

\subsubsection{Phosphorous}

Twenty-three of the inclusions are depleted in phosphorous relative to CI; sixteen are mostly or entirely depleted, and seven are moderately depleted. Phosphates exist in most of the inclusions, usually as a relatively minor phase, $\leq 0.5$ volume $\%$, but $>2.5$ volume percent in two inclusions, and negligible to completely absent in 13 others (see table 3.3).

The lack of phosphates in some inclusions can be explained by the fact that they reside in a high-type, that is, metamorphosed host. Apatite commonly grows during 
metamorphism, and in fact large phosphate grains have been observed in NWA 8232, a type LL5. The inclusion in this meteorite, 8232-I1, has an essentially chondritic bulk composition, except for a stark depletion of phosphorous. Phosphate is present, but only at $0.22 \mathrm{vol} \%$. It is possible that during metamorphism, $\mathrm{P}$ that was present in the inclusion migrated into the host chondrite.

Etr-I1 is rare in that it is enriched in phosphorous, as it contains two anomalously large phosphate grains, one apatite and one merrillite. These phosphate grains appear to have grown within the inclusion, taking up the calcium from nearby pyroxene and feldspar through diffusion as the inclusion material was metamorphosed. Another inclusion, 7869-I1, is also enriched in P. The phosphates present in the inclusion largely occur as rims around metal and sulfide blebs. This suggests that the metal in the inclusion may have once been in a highly reducing environment; phosphorous is siderophile in a low $\mathrm{fO}_{2}$ environment (Huss et al., 2006), which would have caused the $\mathrm{P}$ to enter the metallic phase, and could have later migrated out to form

phosphate minerals when the environment changed. All other inclusions in this study are depleted in metal and sulfide; many are also depleted in P. This may imply that the inclusions formed in a reducing environment, and that phosphorous may have left the inclusions with the metal and sulfide.

\subsection{Olivine "donuts"}

Several inclusions with an olivine-dominated microporphyritic texture contain round, presumably spherical, hollow shells of olivine. These shells, called olivine "donuts" by Jamsja and Ruzicka (2010), are optically aligned single crystals that contain silicate assemblages (olivine, feldspar, and/or pyroxene) in their center. They have been 
observed in five of the inclusions in this study, most of which have between one and five, though NWA 4959-I4 contains over 100, accounting for $\sim 10 \%$ of the inclusion's volume.

These "donuts" are difficult to explain. They are somewhat variable in internal texture; some, like barred olivine chondrules, have plates of olivine extending across their interior. Others, particularly the smaller "donuts," do not have bars but are just shells of olivine with a mixture of silicates of variable grain sizes in the center (see figure 4.12).

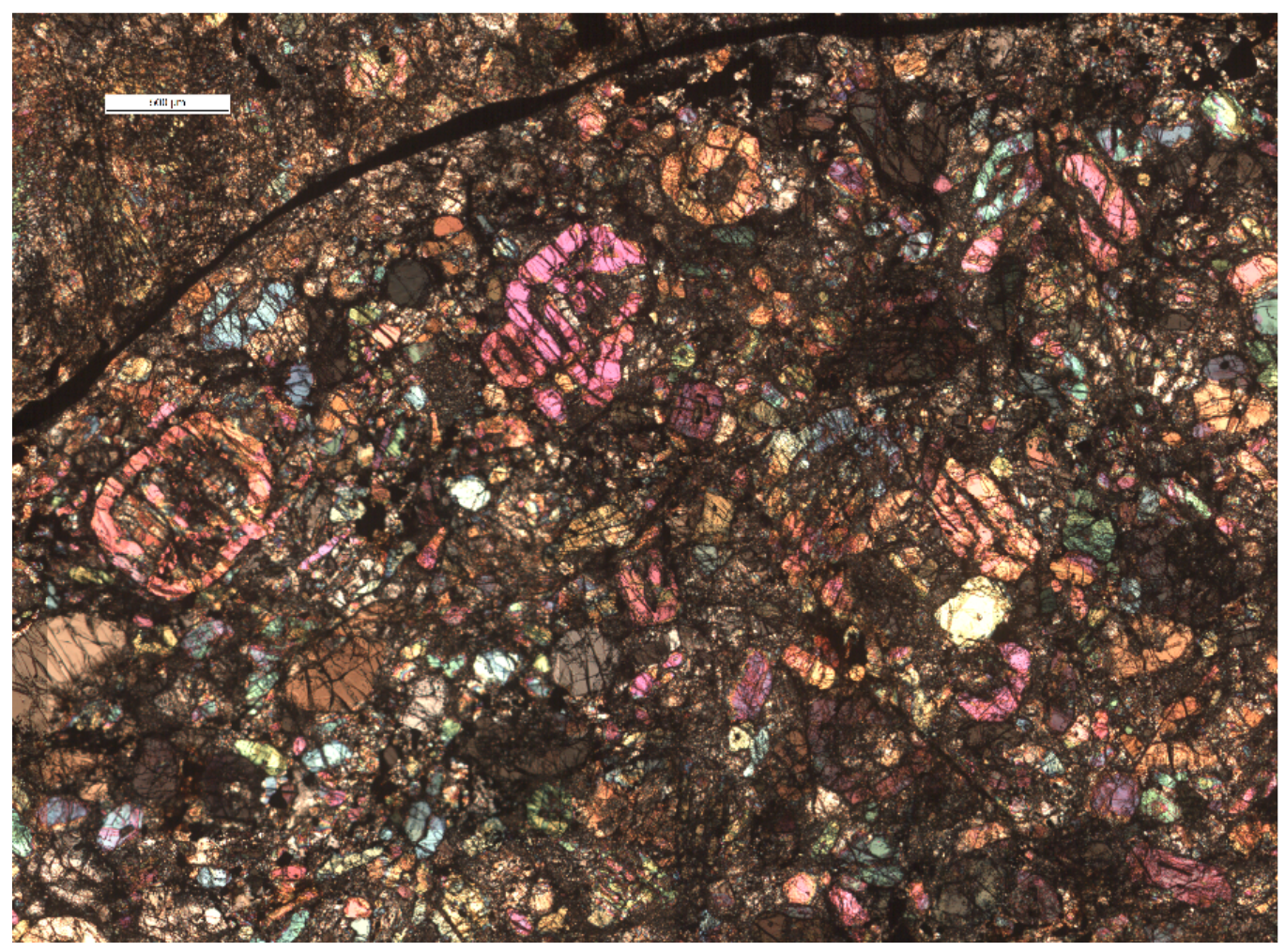

Figure 4.12: Olivine donuts

These objects could either be relict barred olivine chondrules that were part of the precursor material and did not fully melt, or they could have nucleated and crys- 
tallized within the melt. Both of these explanations are problematic. Experiments (Lofgren and Lanier, 1990; Miura et al., 2011) have constrained the formation conditions of barred olivine chondrules, and indicate that to obtain the barred olivine texture, single-crystal shells and plates, the precursor material must be a fully molten and supercooled droplet. It is unclear how this texture could arise from within a larger melt volume. Perhaps a temperature gradient large enough for supercooling could be established if a cool, unmelted chondrule were embedded in melt.

4859-I4, on the other hand, presents a problem with the possibility that the "donuts" are relict barred olivine chondrules: there are too many of them. Surveys of chondrules indicate that BO chondrules only account for $\sim 2-5 \%$ of chondrules (Gooding and Keil, 1981; Weisberg, 1987); therefore, they should account for, at most, $4 \%$ of a chondrite's volume (given an $\mathrm{O}$ chondrite that is $80 \mathrm{vol} \%$ chondrules, (Brearley and Jones, 1998)). So if the "donuts" in 4859-I4 are relict BO chondrules, there must have been some process that concentrated them in the region that was sampled in this PTS.

\subsection{Nearby metal nodules}

With only one exception in this study (7869-I1), large igneous inclusions are highly depleted in metal and sulfide. Three inclusions- PVM-I1, 8231-I1, and 7873-I1- in this study are in close proximity to anomalously large metal and sulfide grains (see figure 4.13), and two others, Glk-I1 and 4859-I18, contain large metal/sulfide grains (though not large enough for these inclusions to not be depleted). In addition, large metal and sulfide grains are reported by Metzler et al. (2011) in NWA 869.

The inclusion in figure 4.13, 7873-I1, is, in thin section, $43 \mathrm{~mm}^{2}$, and the nearby 
metal nodule is $18 \mathrm{~mm}^{2}$, though the latter appears cut off and is probably larger. Assuming its original size to be $20 \mathrm{~mm}^{2}$, and further assuming that both the inclusion and the metal nodule to be spherical and sampled at its equator, the metal nodule is $\sim 20 \%$ of the total volume of the inclusion and metal. These assumptions are most likely incorrect, but this indicates that the volume of metal is the right order of magnitude to have been derived from the same, chondritic, precursor as the inclusion. It is thus possible that these nearby grains are the metal and sulfide that was lost from the inclusion material, and that further study may illuminate the metal/silicate separation process. 


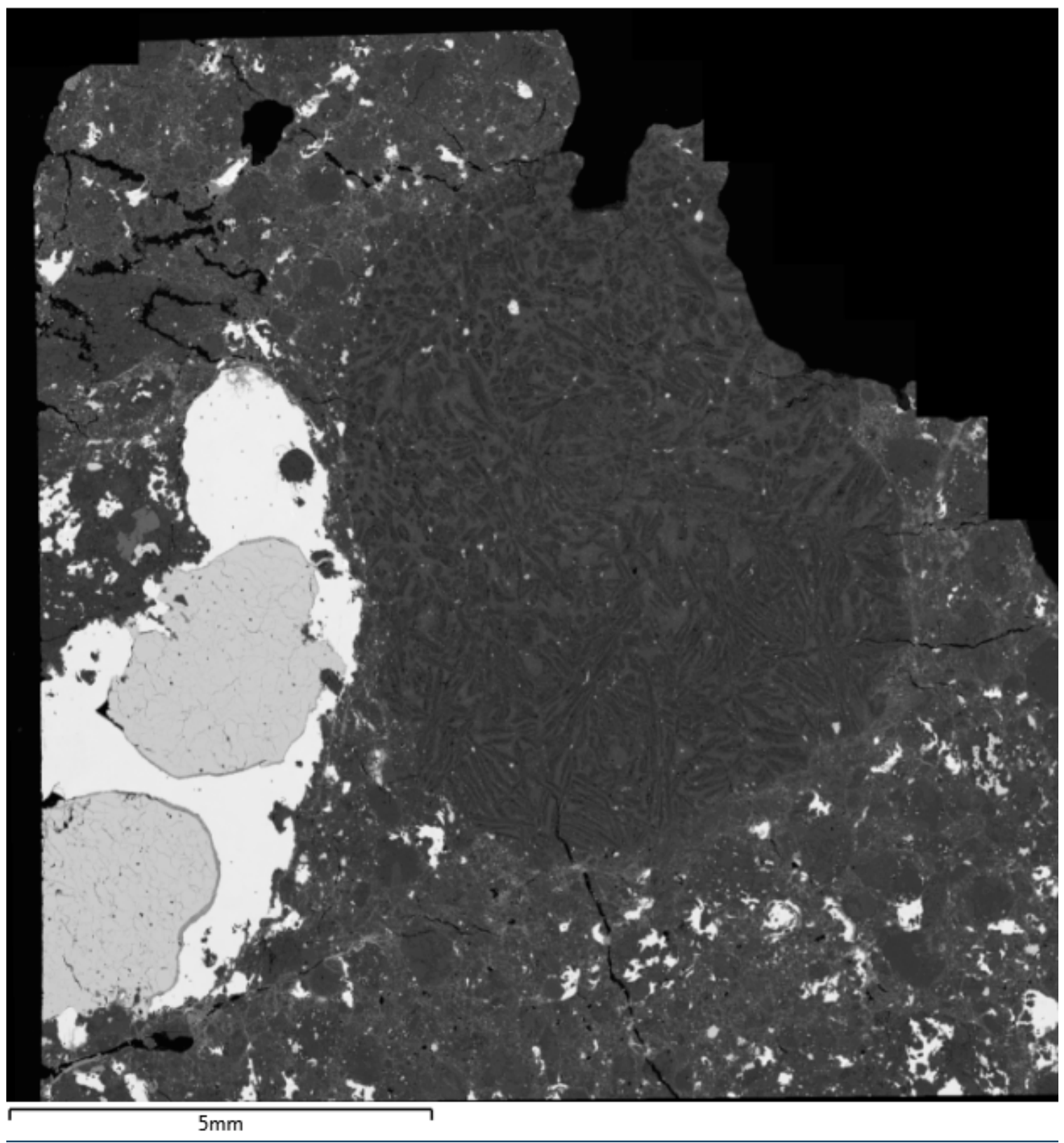

Figure 4.13: BSE micrograph of 7873-I1. This inclusion, like all but one in this study, is clearly depleted in metal (white) and sulfide compared to its host meteorite. Adjacent to this inclusion is an anomalously large metal and sulfide nodule. It is possible the nodule comprises the metal and sulfide that was lost from the inclusion; further study may constrain their relationship. 


\subsection{Brecciation}

Some of the inclusions show evidence of multiple episodes of heating and/or brecciation (see figure 4.14). This includes fractures that do not extend into the host, broken and offset phenocrysts, and phase overgrowths. In the mesostasis of 7873-I1, some of the microlites may have grown after the offset of the crystals (figure 4.15).

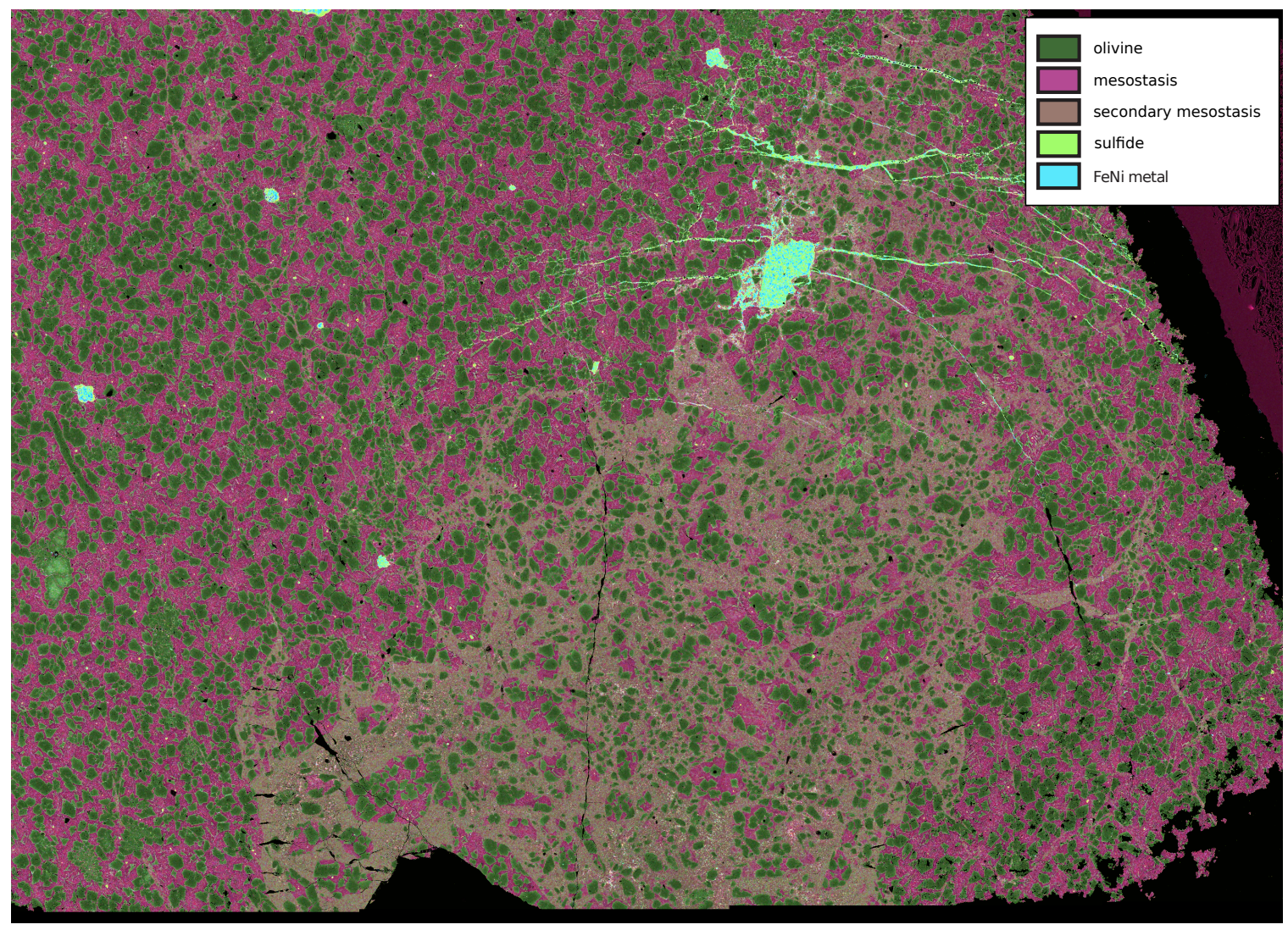

Figure 4.14: False-color EDS phase map of a zone of secondary melt/brecciation in GlkI1. The inclusion has a primary texture of euhedral olivine crystals (green) in a glassy mesostasis (pink). In this region, broken clasts of the inclusion material, as well as single olivine crystals, are suspended in a secondary mesostasis that is richer in $\mathrm{Mg}$ (brown). 


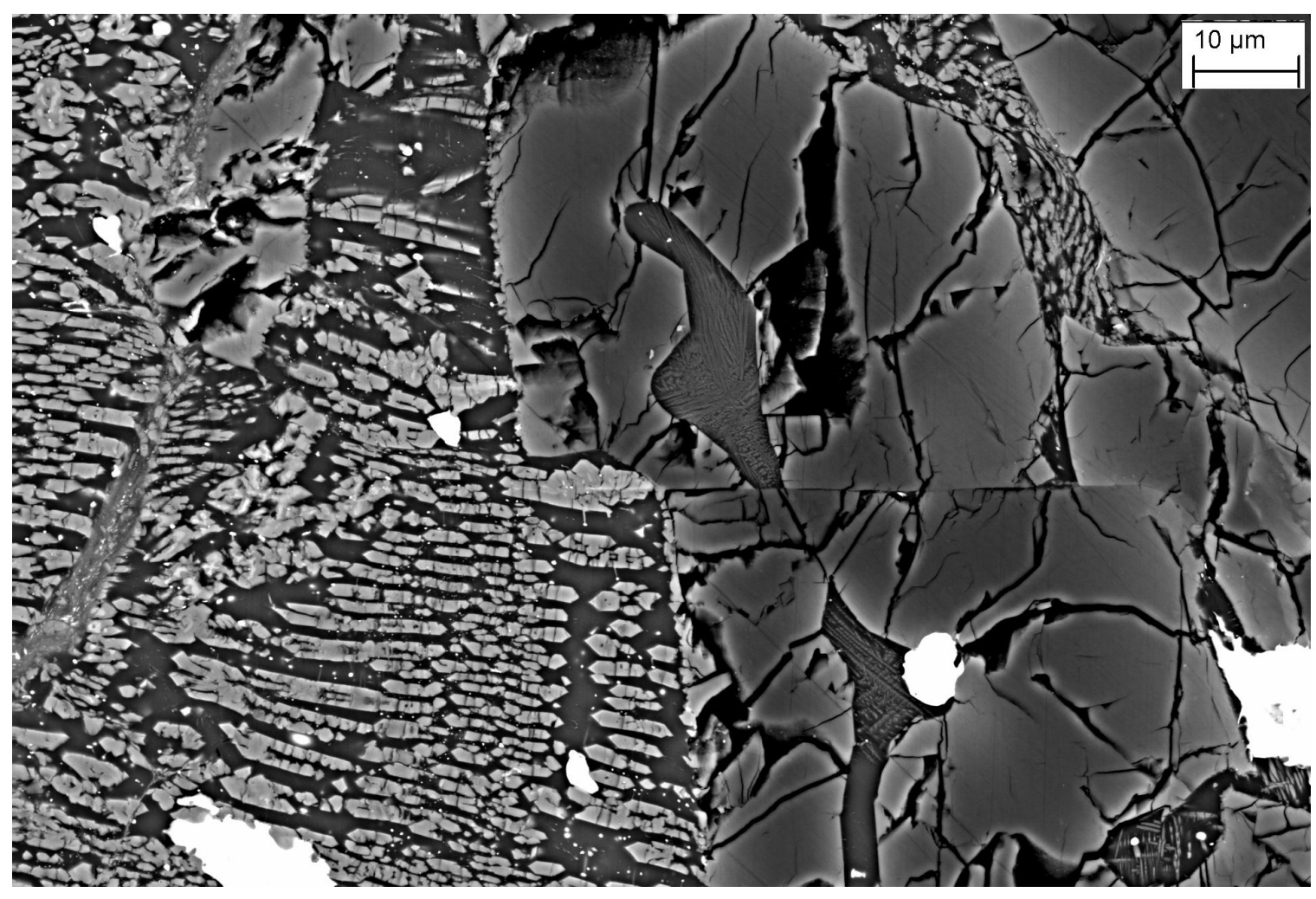

Figure 4.15: BSE micrograph of a broken and offset olivine phenocryst in 7873-I1. Microlites in the glassy mesostasis may have grown after this offset occurred, suggesting multiple heating/brecciation events. 
5 Summary and Conclusions

\section{Summary and Conclusions}

This diverse array of igneous inclusions show evidence for a variety of processes. Overall, they are all largely chondritic, some with signatures of vapor fractionation or shock. Several provide convincing evidence of having crystallized as free-floating droplets. There is no correlation between host meteorite type and any of the trends observed, indicating that the processes at work were not unique to one parent body.

There is also no evident correlation between host equilibration and inclusion equilibration. That is, there are equilibrated hosts with unequilibrated inclusions, and equilibrated inclusions within unequilibrated hosts, implying that the mixing of material occurred over long timescales while metamorphism was ongoing.

None of the inclusions appear to provide any evidence of having originated as an igneous differentiates. None have bulk compositions that approach a cotectic or peritectic, nor do any appear to be residues from partial melting. (One possible exception is Obl-I1, but only a small fragment of this inclusion was available for study and is possibly unrepresentative).

Chemically, the inclusions form three groups that are broadly compatible with the other two proposed formation models, impact melting and large chondrule melt production. In the former group are inclusions that are unfractionated, and essentially chondritic in bulk chemistry. Many of these inclusions also have textural or other evidence of shock processes, such as brecciation, nearby shock veins or shock darkening, or irregular, angular shapes. A nearby metal/sulfide nodule may be material that was removed from the inclusion, possibly via immiscibility after shock heating. The few inclusions that are either enriched or depleted in feldspar may also have been shock 
melts.

There is also evidence that may of these inclusions are megachondrules, and formed via the same processes as chondrules. Most of the clearly drop-formed inclusions had vapor fractionated bulk compositions, though six inclusions that have no evidence of having been an independent object are also vapor fractionated. The vapor-fractionated inclusions can be further subdivided: refractory enriched, volatile depleted, both refractory enriched and volatile depleted, and enriched in elements of mid-volatility. Those inclusions with the strongest evidence for metasomatism are also drop-formed.

Broadly, these results support the conclusions of Ruzicka et al. (1998, 2000), with the Na-poor and Na-rich groups of those studies corresponding to the vaporfractionated and unfractionated groups, respectively. The data presented here can inform and guide future studies of these inclusions, helping to understand their origins and history in the context of other early solar system material. 


\section{References Cited}

Anders, E. and Grevesse, N., 1989. Abundances of the elements: Meteoritic and solar. Geochimica et Cosmochimica Acta, 53(1): 197-214.

Binns, R. A., 1967. An exceptionally large chondrule in the Parnallee meteorite. Mineralogical Magazine, 36(279): 319-324.

Brearley, A. J. and Jones, R. H., 1998. Chondritic meteorites. Reviews in Mineralogy and Geochemistry, 36(1): 3.1-3.398.

Bridges, J. C. and Hutchison, R., 1997. A survey of clasts and large chondrules in ordinary chondrites. Meteoritics $\& 3$ Planetary Science, 32(3): 389-394.

Burbine, T. H., McCoy, T. J., Meibom, A., Gladman, B., and Keil, K., 2002. Meteoritic parent bodies: Their number and identification. In Bottke, W. F., Jr., Cellino, A., Paolicchi, P., and Binzel, R., editors, Asteroids III, pages 653-667. University of Arizona Press.

Chen, M. and El Goresy, A., 2000. The nature of maskelynite in shocked meteorites: not diaplectic glass but a glass quenched from shock-induced dense melt at high pressures. Earth and Planetary Science Letters, 179(3-4): 489-502.

Davis, A. M., 2006. Volatile evolution and loss. In Lauretta, D. S. and Mcsween, H. Y., Jr, editors, Meteorites and the Early Solar System II, pages 295-307. University of Arizona Press.

Dodd, R. T. and Jarosewich, E., 1976. Olivine microporphyry in the St. Mesmin chondrite. Meteoritics, 11(1): 1-20. 
Dodd, R. T. and Jarosewich, E., 1979. Incipient melting in and shock classification of L-group chondrites. Earth and Planetary Science Letters, 44(2): 335-340.

Fodor, R. V. and Keil, K., 1976. Carbonaceous and non-carbonaceous lithic fragments in the Plainview, Texas, chondrite: origin and history. Geochimica et Cosmochimica Acta, 40(2): 177-189.

Friedrich, J. M., Weisberg, M. K., Ebel, D. S., Biltz, A. E., Corbett, B. M., Iotzov, I. V., Khan, W. S., and Wolman, M. D., 2014. Chondrule size and related physical properties: A compilation and evaluation of current data across all meteorite groups. Chemie der Erde - Geochemistry, in press.

Gooding, J. L. and Keil, K., 1981. Relative abundances of chondrule primary textural types in ordinary chondrites and their bearing on conditions of chondrule formation. Meteoritics, 16(1): 17-43.

Hewins, R. H., 1997. Chondrules. Annual Review of Earth and Planetary Sciences, 25(1): $61-83$.

Huss, G. R., Rubin, A. E., and Grossman, J. N., 2006. Thermal metamorphism in chondrites. In Lauretta, D. S. and Mcsween, H. Y., Jr, editors, Meteorites and the Early Solar System II, pages 567-586. University of Arizona Press.

Hutchison, R., 2004. Meteorites: A Petrologic, Chemical and Isotopic Synthesis. Cambridge planetary science series. Cambridge University Press.

Hutchison, R., Williams, C. T., Din, V. K., Clayton, R. N., Kirschbaum, C., Paul, R. L., and Lipschutz, M. E., 1988. A planetary, H-group pebble in the Barwell, 
L6, unshocked chondritic meteorite. Earth and Planetary Science Letters, 90(2): $105-118$.

Jamsja, N. and Ruzicka, A., 2010. Shock and thermal history of northwest africa 4859, an annealed impact-melt breccia of LL chondrite parentage containing unusual igneous features and pentlandite. Meteoritics \& Planetary Science, 45(5): 828849.

Jones, R. H., Grossman, J. N., and Rubin, A. E., 2005. Chemical, mineralogical and isotopic properties of chondrules: Clues to their origin. In Krot, A. N., Scott, E. R. D., and Reipurth, B., editors, Chondrites and the Protoplanetary Disk.

Kieffer, S. W., 1975. From regolith to rock by shock. Earth, Moon, and Planets, 13(1-3): 301-320.

Klein, C. and Dutrow, B., 2007. Manual of Mineral Science. Wiley.

Lauretta, D. S., Nagahara, H., and Alexander, C. M. O., 2006. Petrology and origin of ferromagnesian silicate chondrules. In Lauretta, D. S. and Mcsween, H. Y., Jr, editors, Meteorites and the Early Solar System II. University of Arizona Press.

Lodders, K., 2003. Solar system abundances and condensation temperatures of the elements. Asian Pacific journal of cancer prevention: APJCP, 591(2): 1220.

Lofgren, G. and Lanier, A. B., 1990. Dynamic crystallization study of barred olivine chondrules. Geochimica et Cosmochimica Acta, 54(12): 3537-3551.

McSween, H., 1987. Meteorites and their parent planets. Cambridge University Press. 
Melosh, H. J., 1989. Impact cratering: A geologic process. Oxford University Press.

Metzler, K., Bischoff, A., Greenwood, R. C., Palme, H., Gellissen, M., Hopp, J., Franchi, I. A., and Trieloff, M., 2011. The L3-6 chondritic regolith breccia Northwest Africa (NWA) 869: (I) Petrology, chemistry, oxygen isotopes, and Ar-Ar age determinations. Meteoritics \& Planetary Science, 46(5): 652-680.

Miura, H., Yokoyama, E., Nagashima, K., Tsukamoto, K., and Srivastava, A., 2011. A new constraint for chondrule formation: condition for the rim formation of barredolivine textures. Earth Planets Space, 63(10): 1087-1096.

Prinz, M., Weisberg, M. K., and Nehru, C. E., 1988. Gunlock: A new type 3 ordinary chondrite with a golfball-sized chondrule. Meteoritics, 23: 297.

Rubin, A. E., Keil, K., Taylor, G. J., Ma, M.-S., Schmitt, R. A., and Bogard, D. D., 1981. Derivation of a heterogeneous lithic fragment in the Bovedy L-group chondrite from impact-melted porphyritic chondrules. Geochimica et Cosmochimica Acta, 45(11): 2213-2228.

Ruzicka, A. and Hutson, M., 2006. Differentiation and evolution of the IVA meteorite parent body: Clues from pyroxene geochemistry in the Steinbach stony-iron meteorite. Meteoritics \& Planetary Science, 41(12): 1959-1987.

Ruzicka, A. and Hutson, M., 2010. Comparative petrology of silicates in the Udei Station (IAB) and Miles (IIE) iron meteorites: Implications for the origin of silicatebearing irons. Geochimica et Cosmochimica Acta, 74(1): 394-433.

Ruzicka, A., Kring, D. A., Hill, D. H., Boynton, W. V., Clayton, R. N., and Mayeda, 
T. K., 1995. Silica-rich orthopyroxenite in the Bovedy chondrite. Meteoritics, 30(1): $57-70$.

Ruzicka, A., Snyder, G. A., and Taylor, L. A., 1998. Mega-chondrules and large, igneous-textured clasts in Julesberg (L3) and other ordinary chondrites: vaporfractionation, shock-melting, and chondrule formation. Geochimica et Cosmochimica Acta, 62(8): 1419-1442.

Ruzicka, A., Fowler, G. W., Snyder, G. A., Prinz, M., Papike, J. J., and Taylor, L. A., 1999. Petrogenesis of silicate inclusions in the Weekeroo Station IIE iron meteorite: Differentiation, remelting, and dynamic mixing. Geochimica et Cosmochimica Acta, 16(13/14): 2123-2143.

Ruzicka, A., Snyder, G. A., and Taylor, L. A., 2000. Geochemical and isotopic evidence bearing on the origin of large, igneous-textured inclusions in ordinary chondrites. Antarctic Meteorite Research, 13: 19-38.

Ruzicka, A., Hutson, M., Floss, C., and Hildebrand, A., 2012a. Large silica-rich igneous-textured inclusions in the Buzzard Coulee chondrite: Condensates, differentiates, or impact melts? Meteoritics $\&$ Planetary Science, 47(11): 1809-1829.

Ruzicka, A., Hutson, M., Floss, C., and Hildebrand, A., 2012b. Large Silica-Rich Igneous-Textured inclusions in the Buzzard Coulee (H4) chondrite. In Lunar and Planetary Institute Science Conference Abstracts, volume 43, page 1630.

Sears, D. W., Grossman, J. N., Melcher, C. L., Ross, L. M., and Mills, A. A., 1980. Measuring metamorphic history of unequilibrated ordinary chondrites. Nature, 287: 791-795. 
Sears, D. W. G. and Dodd, R. T., 1988. Overview and classification of meteorites. In Lauretta, D. S. and Mcsween, H. Y., Jr, editors, Meteorites and the Early Solar System II. University of Arizona Press.

Sharp, T. G. and DeCarli, P. S., 2006. Shock effects in meteorites. In Lauretta, D. S. and Mcsween, H. Y., Jr, editors, Meteorites and the Early Solar System II. University of Arizona Press.

Stöffler, D., Keil, K., and Edward R.D, S., 1991. Shock metamorphism of ordinary chondrites. Geochimica et Cosmochimica Acta, 55(12): 3845-3867.

Van Schmus, W. R. and Wood, J. A., 1967. A chemical-petrologic classification for the chondritic meteorites. Geochimica et Cosmochimica Acta, 31(5): 747-765.

Weisberg, M. K., 1987. Barred olivine chondrules in ordinary chondrites. Journal of Geophysical Research [Solid Earth], 92(B4): E663-E678.

Weisberg, M. K., Prinz, M., and Nehru, C. E., 1988. Macrochondrules in ordinary chondrites: Constraints on chondrule forming processes. Meteoritics, 23: 309.

Weisberg, M. K., McCoy, T. J., and Krot, A. N., 2006. Systematics and evaluation of meteorite classification. In Lauretta, D. S. and McSween, H. Y., Jr., editors, Meteorites and the Early Solar System II. University of Arizona Press.

Weyrauch, M. and Bischoff, A., 2012. Macrochondrules in chondrites-Formation by melting of mega-sized dust aggregates and/or by rapid collisions at high temperatures? Meteoritics 83 Planetary Science, 47(12): 2237-2250. 
Wood, J. A., 1996. Unresolved issues in the formation of chondrules and chondrites. In Hewins, R. H., Jones, R. H., and Scott, E. R. D., editors, Chondrules and the Protoplanetary Disk. Cambridge University Press.

Wood, J. A., 2005. The chondrite types and their origins. In Krot, A. N., Scott, E. R. D., and Reipurth, B., editors, Chondrites and the Protoplanetary Disk, pages 953-971. 


\section{Appendix A Chemical and petrographic descriptions of indi- vidual inclusions}

\section{A.1 NWA 8231 (CML 0368) H4-6}

NWA 8231 is an H4-6 breccia. One polished thin section containing one exceptionally large inclusion has been examined.

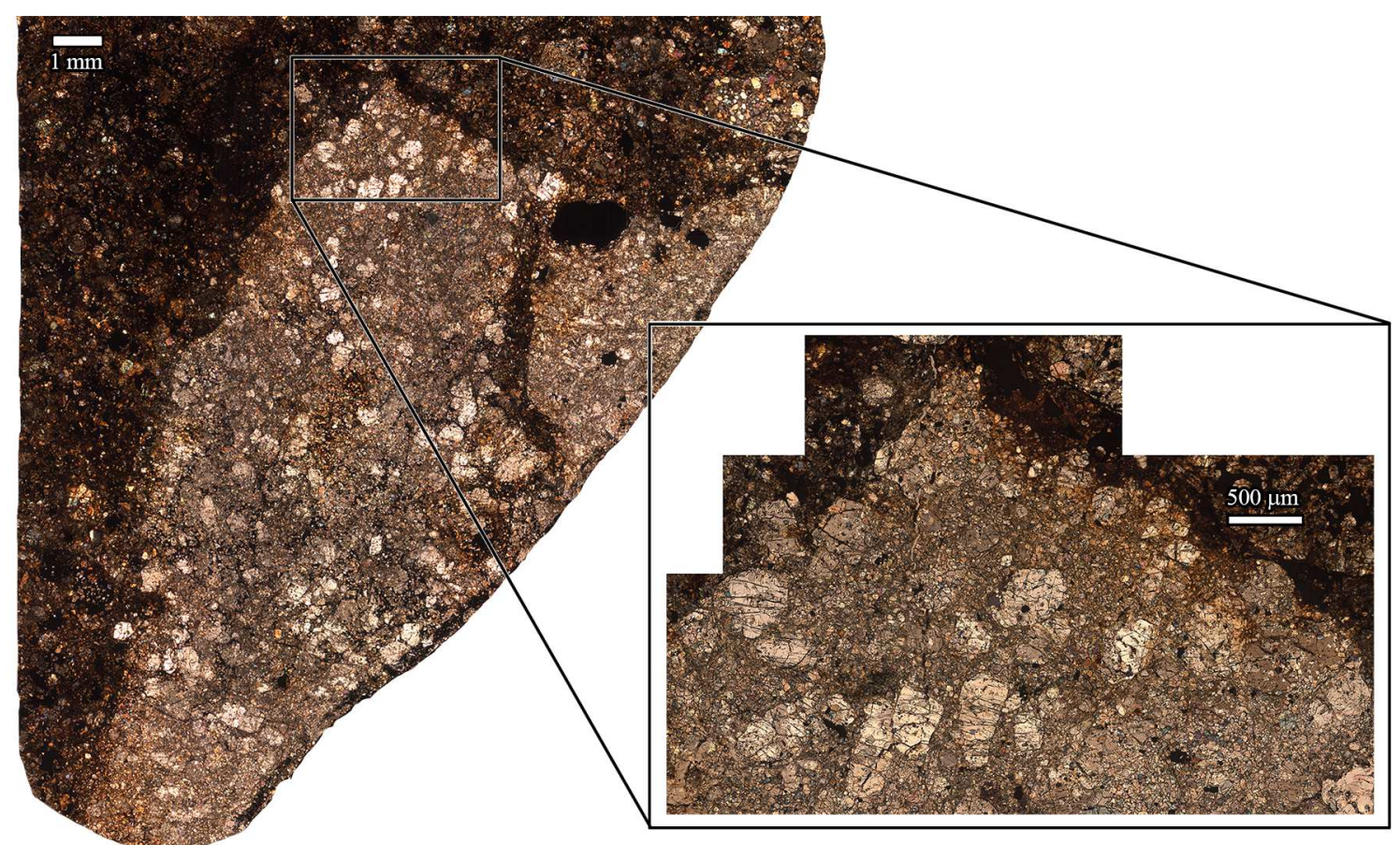

Figure A.1: 8231-I1 under cross-polarized light. Large phenocrysts are low-Ca pyroxene, with olivine poikilitically enclosed and interstitial. Opaque sulfide is visible only in the center of the inclusion, and an anomalously large metal/sulfide nodule is at the host contact.

0368-I1 This inclusion is very large, and is truncated at the edge of the sample, so original size is unknown. However, its greatest apparent length is $>18 \mathrm{~mm}$, so it may have been several centimeters in diameter. Its contact with the host is defined 
A Chemical and petrographic descriptions of individual inclusions

by an opaque vein of shock-melted material. It is an angular contact, though large crystals are not broken, but protrude, suggesting the inclusion has been abraded or eroded. It has a poikilitic texture, with coarse $(300-500 \mu \mathrm{m})$ subhedral grains of lowCa pyroxene enclosing smaller olivine grains. Small $(20-100 \mu \mathrm{m})$, anhedral olivine, anhedral diopside, and anhedral feldspar is interstitial to the low-Ca pyroxene.

The inclusion has three regions that vary slightly in mineralogy and texture. The center of the inclusion has a substantial amount of sulfide, metal and phosphate interstitial to the silicates, which is mostly absent from the majority of the inclusion. Additionally, there are numerous anomalously coarse metal grains in proximity to this inclusion. A fragment of the inclusion is separated by a vein of shock-melted material, and this fragment has the same mineralogy and texture as the rest of the inclusion, but the low-Ca pyroxene grains are about half the size. The inclusion is one of many that appear crossed by fracture zones that do not continue into the host, suggesting it may have been brecciated before being incorporated into the host.

The olivine and low-Ca pyroxene are highly equilibrated, with Fa $21.0 \pm 0.2(\mathrm{~N}$ $=17)$, and Wo $2.7 \pm 0.6$, Fs $17.6 \pm 0.4$, En $79.6 \pm 0.8(\mathrm{~N}=14)$, both of which are average for $\mathrm{H}$ chondrites. The diopside is more variable, with average composition Wo $38.1 \pm 1.7$, Fs $11.2 \pm 1.3$, En $50.7 \pm 0.8$ (Determined with AZtec's analyze phases). The feldspar is K-rich, and somewhat variable, with average composition Ab $82.7 \pm$ 1.6, Or $13.6 \pm 1.9$, An $3.7 \pm 1.9(\mathrm{~N}=8)$. Olivine, low-Ca pyroxene, and chromite composition was determined by EDS point analyses, feldspar was determined by a mixture of EDS point spectra and EDS map extracts. Diopside, metal, sulfide, and phosphate was determined with AZtec's analyze phases.

This inclusion is one of several that has an anomalously large metal and sulfide 
grain in close proximity. This, and the uneven distribution of metal and sulfide within the inclusion itself, suggests that further study of the metal may illuminate the process that separate metal and sulfide from silicates, resulting in these inclusions being depleted in metal.

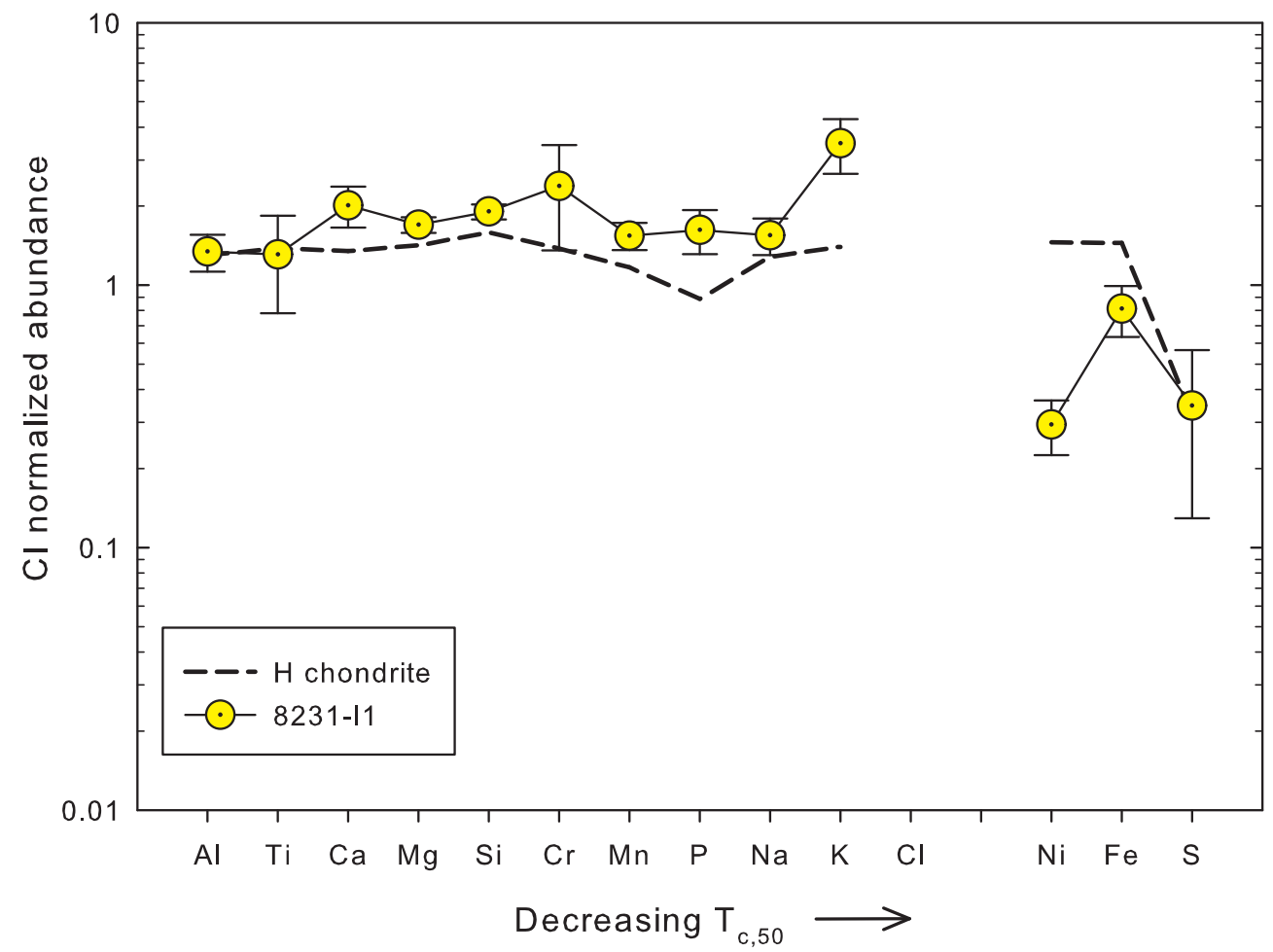

Figure A.2: CI-normalized bulk chemistry of 8231-I1, with reference H chondrite (host meteorite). This analysis does not include a nearby coarse metal and sulfide grain. Notable is an enrichment in potassium, without an enrichment in sodium or aluminum, suggesting the $\mathrm{K}$ enrichment does not arise from a feldspar enrichment or metasomatism (see section 4.2.1). 
A Chemical and petrographic descriptions of individual inclusions

\section{A.2 Tamdakht (CML 0646) H5}

Tamdakht is an H5 chondrite. Two inclusions in two separate thin sections were examined.

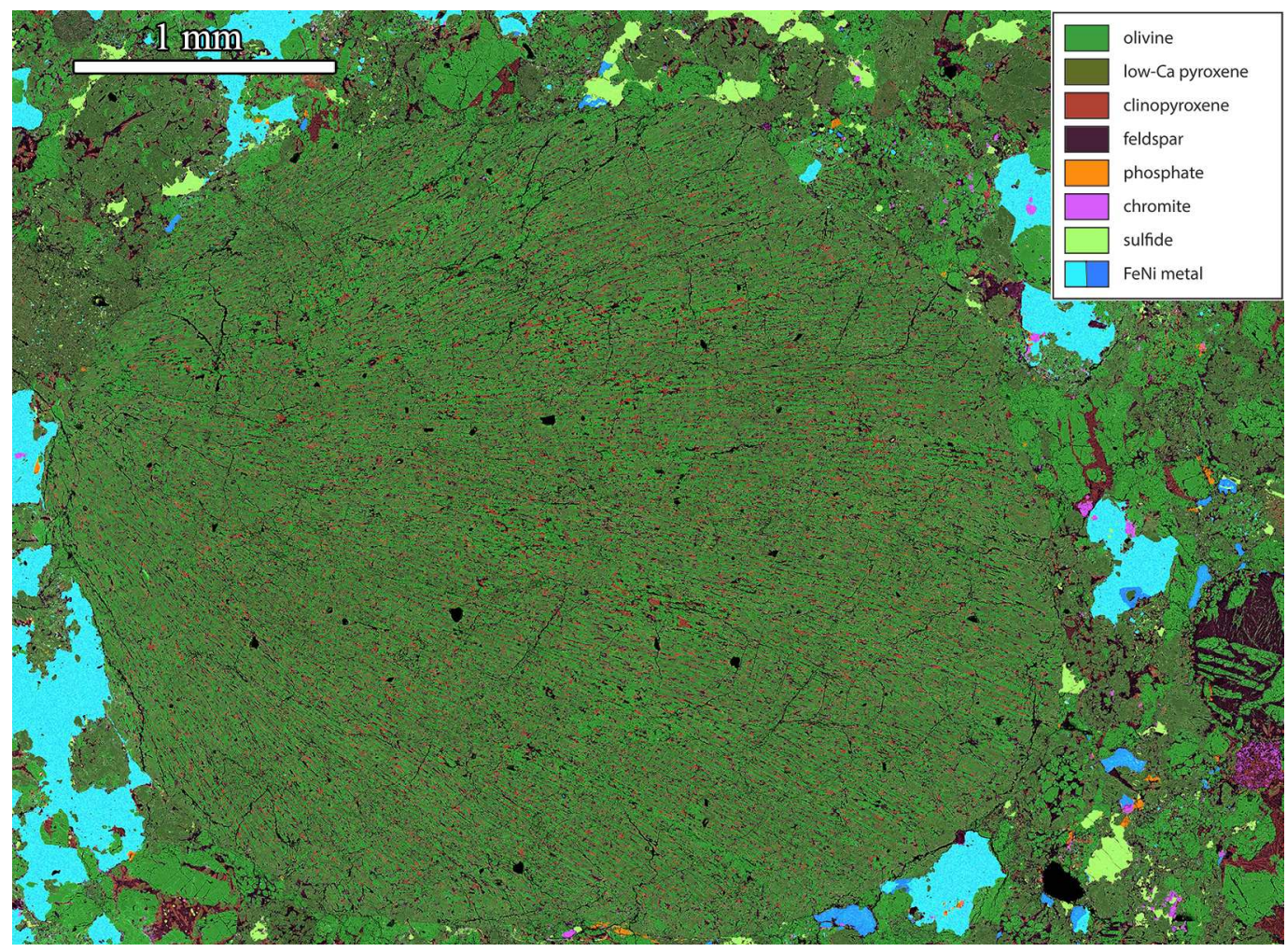

Figure A.3: False-color EDS phase map of Tdk-I1. (See figure 4.1 for plane-transmitted light image).

Tdk-I1 The first inclusion is rounded and slightly oblate; $3.25 \times 4 \mathrm{~mm}$ in apparent size. It is in sharp contact with the host, with much of the contact defined by a fracture. While some crystals at the edge appear broken, there is a near complete rim of low-Ca pyroxene that is texturally different from the interior of the inclusion, suggesting that this edge is a primary feature. 
A Chemical and petrographic descriptions of individual inclusions

Texturally, the inclusion resembles a radial pyroxene (RP) chondrule. Long, thin plates of intergrown olivine and low-Ca pyroxene extend across the inclusion from one point on the rim. These plates are only $\sim 10 \mu \mathrm{m}$ thick, but can extend across the entire inclusion. Diopside and feldspar are interstitial throughout the inclusion. As mentioned, the rim is dominated by low-Ca pyroxene, which is coarser than the plates. The inclusion is almost entirely devoid of all other phases, with only a few minute blebs of FeNi metal, sulfide, and chromite throughout.

The olivine is highly equilibrated, with average Fa of $18.4 \pm 0.4(\mathrm{~N}=16)$. The low-Ca pyroxene is more variable in composition, with some regions that appear to be zoned. The average composition is Wo $4.1 \pm 1.0$, Fs $15.7 \pm 1.1$, En $80.2 \pm 1.1$ $(\mathrm{N}=22)$. The diposide is more equilibrated, with average composition Wo $48.8 \pm$ 0.6 , Fs $5.6 \pm 0.6$, En $45.6 \pm 0.3$, though it should be noted it was characterized with the Analyze Phases function, which averages all pixels with similar chemistry. Two distinct feldspathic phases are in exolution: a more albitic phase that appears glassy, with average composition Ab $81.2 \pm 2.0$, Or $8.7 \pm 3.8$, An $10.2 \pm 3.4(\mathrm{~N}=4)$, and a more anorthitic phase that appears to have a spongy texture and contain extremely minute $(\sim 100 \mathrm{~nm})$ blebs of chromite that has average composition Ab $46.8 \pm 12.3$, Or $1.3 \pm 0.5$, An $51.9 \pm 12.7(\mathrm{~N}=6)$.

Olivine, low-Ca pyroxene, and both feldspars were determined with EDS point spectra. Diopside was determined with AZtec's analyze phases. Chromite, sulfide, and taenite were all detected but in too small a quantity to characterize, and so were assumed to be pure phases. 
A Chemical and petrographic descriptions of individual inclusions

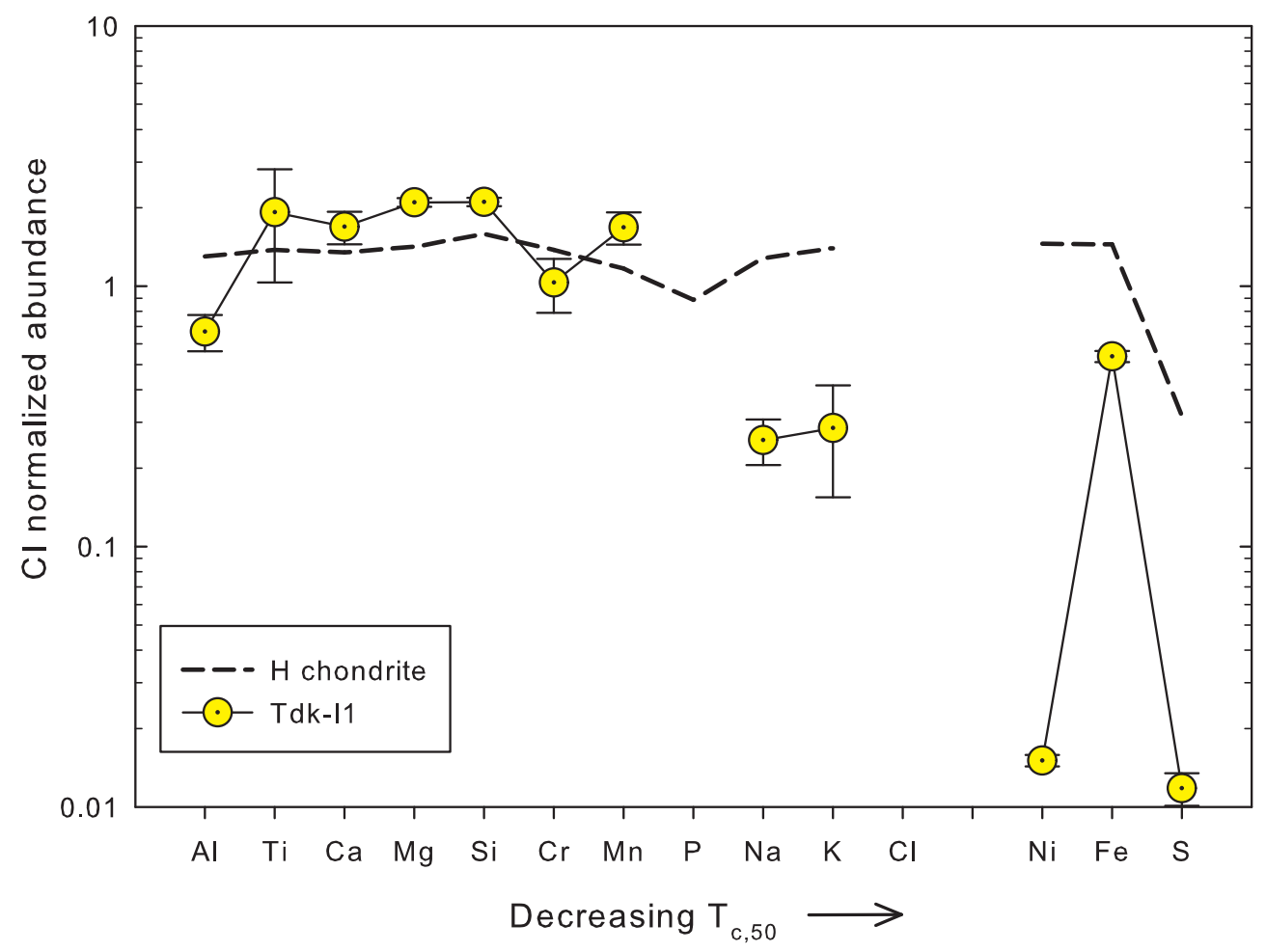

Figure A.4: CI-normalized bulk chemistry of Tdk-I1. This inclusion did not contain any detectable phosphate minerals, and is also depleted in the elements that make up feldspar (see section 4.3.2). 
A Chemical and petrographic descriptions of individual inclusions

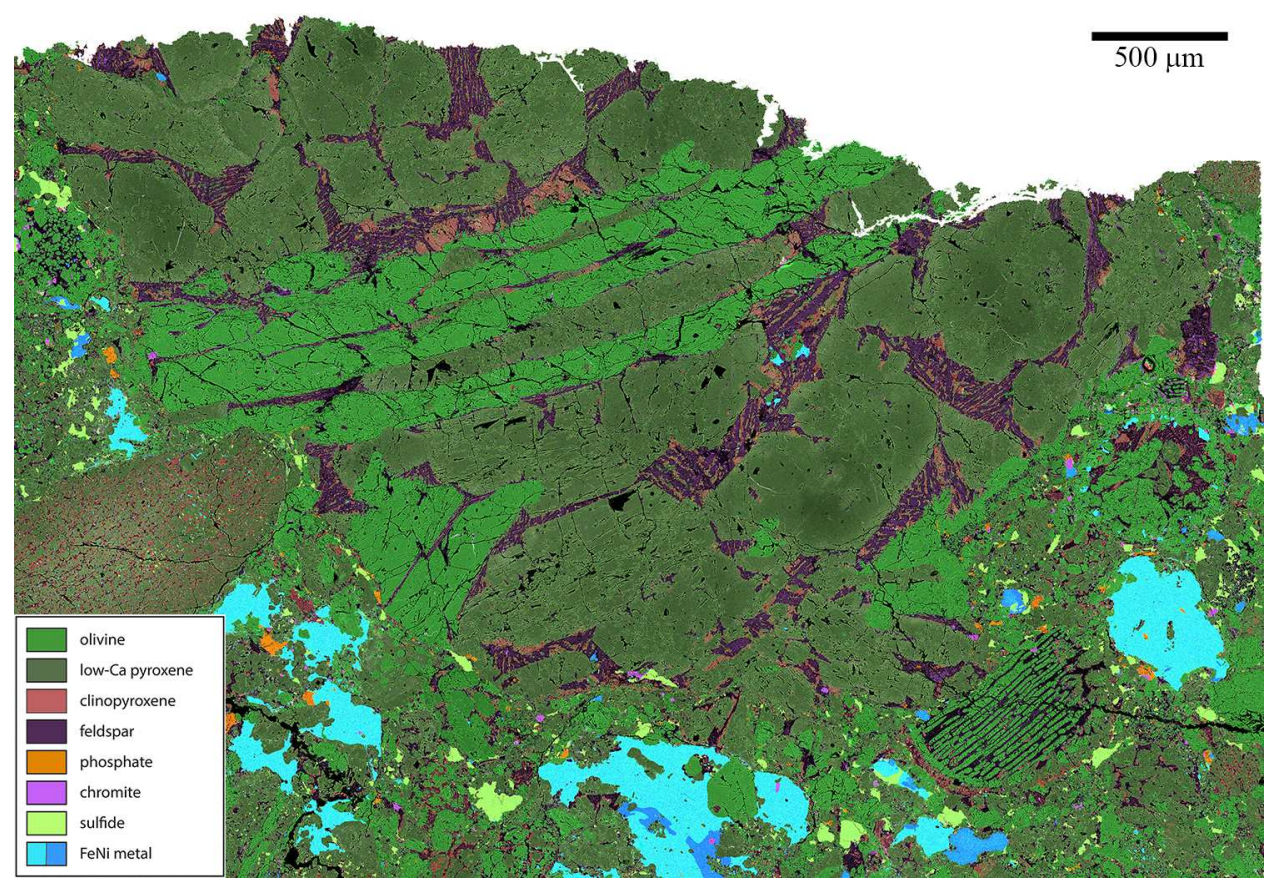

Figure A.5: False-color EDS phase map of Tdk-I2.

Tdk-I2 Only a fragment of this inclusion is available on the thin section, so it may be much larger than its largest observable dimension, which is $5 \mathrm{~mm}$. Its contact with the host is jagged and fairly sharp, with most of the larger crystals at the edge clearly broken off, suggesting that this inclusion is a fragment of a larger igneous object.

This inclusion is an olivine and low-Ca pyroxene microporphyry, with relatively coarse $(100 \mu \mathrm{m}-1 \mathrm{~mm})$ subhedral to euhedral low-Ca pyroxene phenocrysts in a groundmass of intergrown feldspar and diopside. Olivine is present as 3-4 coarse, mutually aligned blades that transect the entire fragment of the inclusion. The feldspar shows exolution lamellae, but is relatively uniform in composition. Chromite, phosphate and sulfite exist as a few rare grains close to the host contact, and small blebs of kamacite and taenite are in the interior. 
A Chemical and petrographic descriptions of individual inclusions

Olivine is equilibrated, with average composition Fa $18.6 \pm 0.3(\mathrm{~N}=11)$. Low-Ca pyroxene is more chemically variable, with average composition Wo $2.2 \pm 1.2$, Fs $16.3 \pm 1.2$, En $81.5 \pm 2.2(\mathrm{~N}=25)$, as the larger phenocrysts are zoned. Diopside is even more variable, averaging Wo $37.1 \pm 5.9$, Fs $8.7 \pm 2.3$, En $54.2 \pm 1.2(\mathrm{~N}=$ 13). The feldspar, despite the exolution lamellae, averages Ab $17.0 \pm 2.3$, Or $0.2 \pm$ 0.1 , An $82.7 \pm 2.3(\mathrm{~N}=12)$. Olivine, both pyroxenes, feldspar, metal, and ilmenite were determined with EDS point spectra. Chromite and sulfide were determined from EDS map extracts. Phosphate was determined with AZtec's analyze phases.

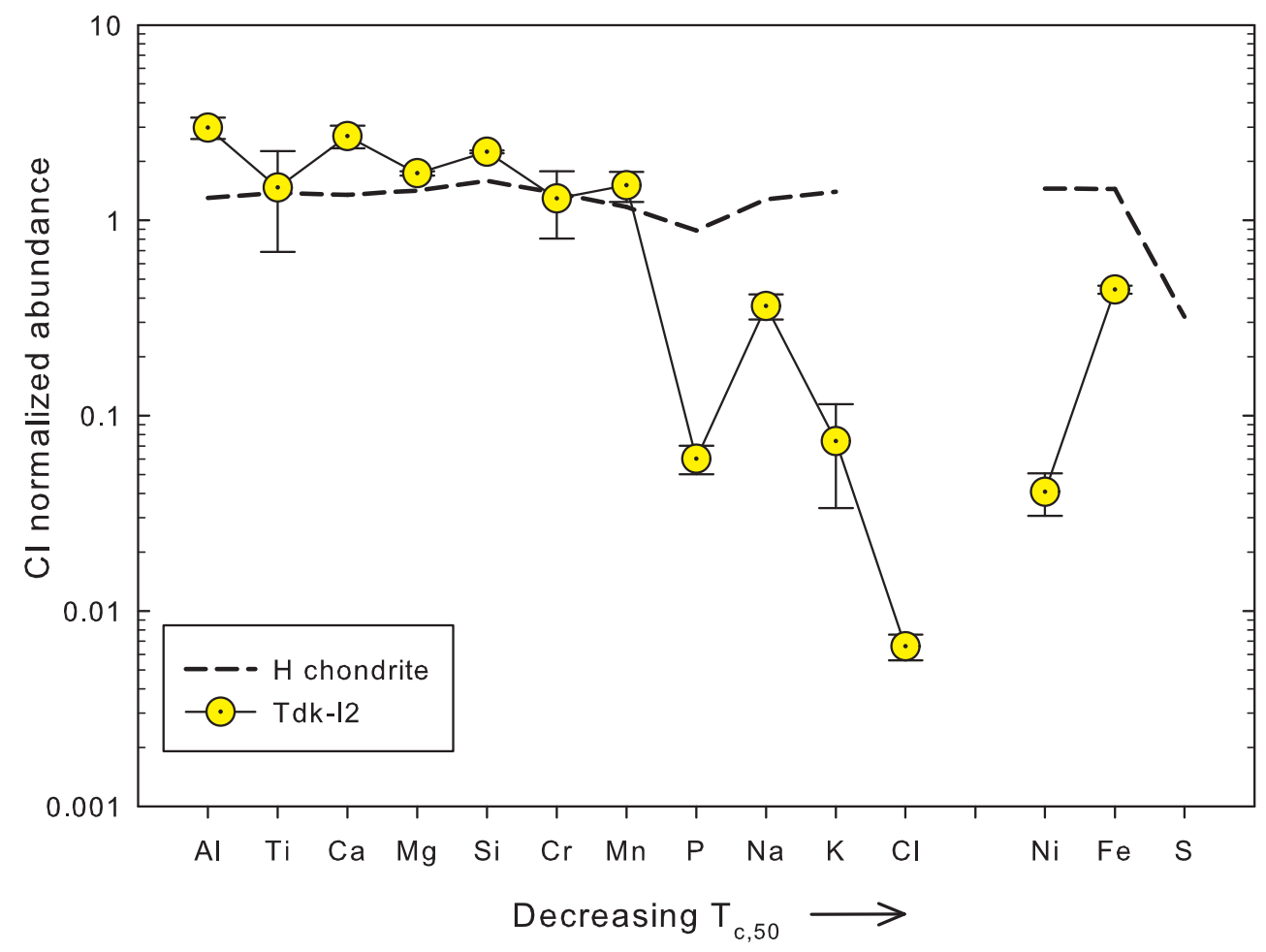

Figure A.6: CI-normalized bulk chemistry of Tdk-I2. This inclusion is depleted in phosphate and sulfide minerals, but is also strongly depleted in volatile elements, suggesting a vaporfractionation process. 
A Chemical and petrographic descriptions of individual inclusions

\section{A.3 NWA 7873 (CML 0615) H5-6}

One PTS of NWA 7873, an H5-6 breccia, which contains one inclusion has been examined.

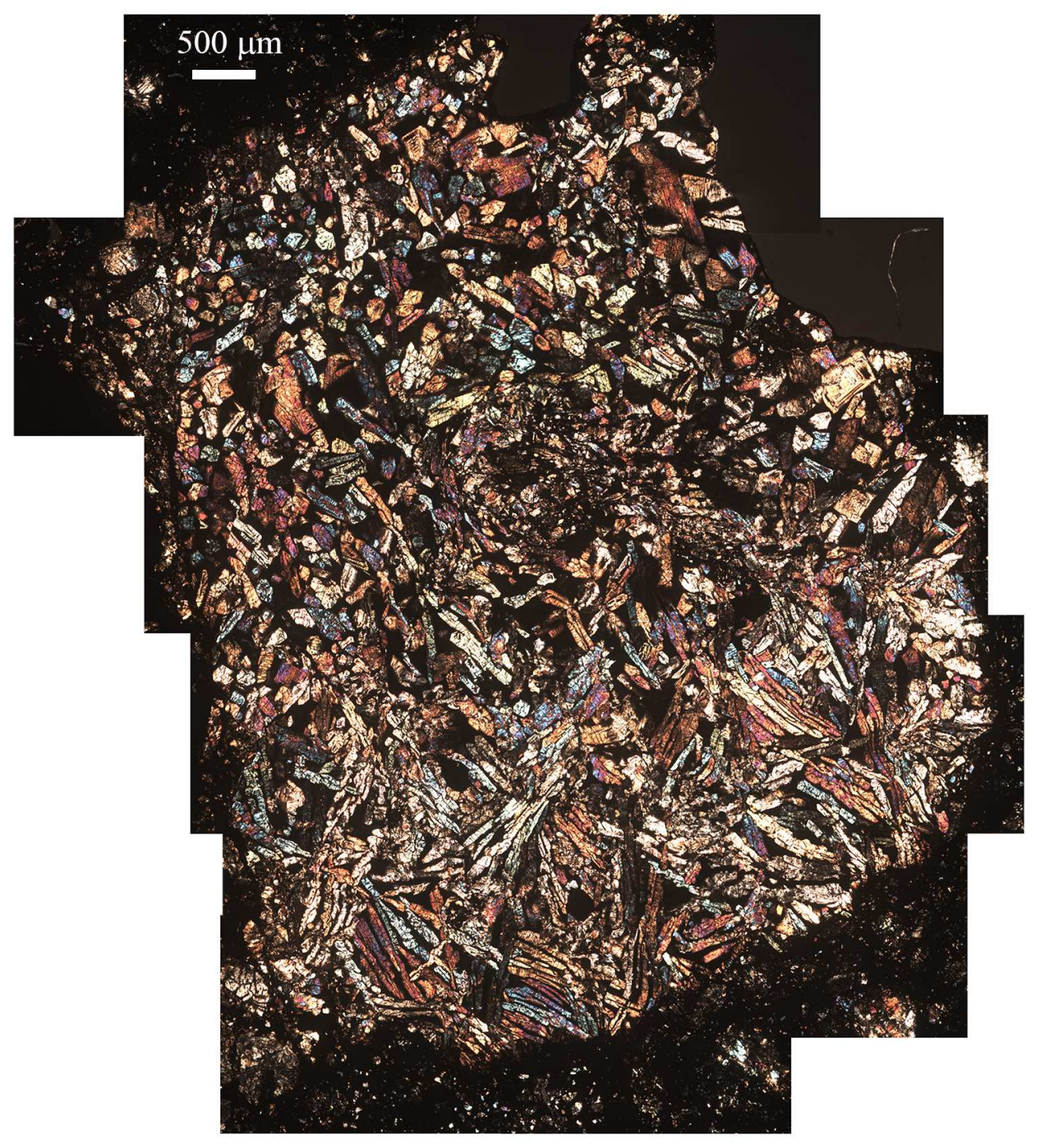

Figure A.7: Cross-polarized optical light micrograph of 7873-I1. 
A Chemical and petrographic descriptions of individual inclusions

NWA 7873-I1 The inclusion is subrounded, and its apparent size is $6 \mathrm{~mm} \times 8 \mathrm{~mm}$. Some crystals at the edge of the sample at the host contact are broken, with a clean, sharply defined edge in contact with host material, suggesting this is a fragment of a larger object.

It is an olivine microporphyry, with euhedral olivine phenocrysts in a crystalline pyroxene/feldspar groundmass. The phenocrysts show an average change in shape, within the inclusion, from shorter to more elongated. The average grain size for the olivines is $150 \mu \mathrm{m}$, with a range of $40 \mu \mathrm{m}$ to $400 \mu \mathrm{m}$.

Chemically, this inclusion is itself unequilibrated while residing in an equilibrated host. The average fayalite content of olivine is $12.4 \pm 3.8(\mathrm{~N}=35)$, which is substantially lower than average $\mathrm{H}$ chondrite. The unequilibration is a result of zoned crystals rather than a radial variation. The groundmass consists of microlites of pigeonite and olivine mantled in pigeonite set in a glassy feldspathic groundmass. The microlites are too small to adequately measure their composition. Olivine was determined with EMPA and EDS point spectra. The mesostasis was determined from EDS map extracts. Metal and sulfide were determined through EDS point spectra and AZtec analyze phases, respectively. Ilmenite is present, but at too small a quantity to be characterized and so was assumed pure.

BSE images reveal that several of the phenocrysts within the inclusion are also broken, with clear offset. Some of this offset appears to have affected the groundmass as well, while in others it appears that the groundmass crystallized after the offset. This inclusion is also one of three that are in close proximity to an anomalously large metal/sulfide grain in the host. 
A Chemical and petrographic descriptions of individual inclusions

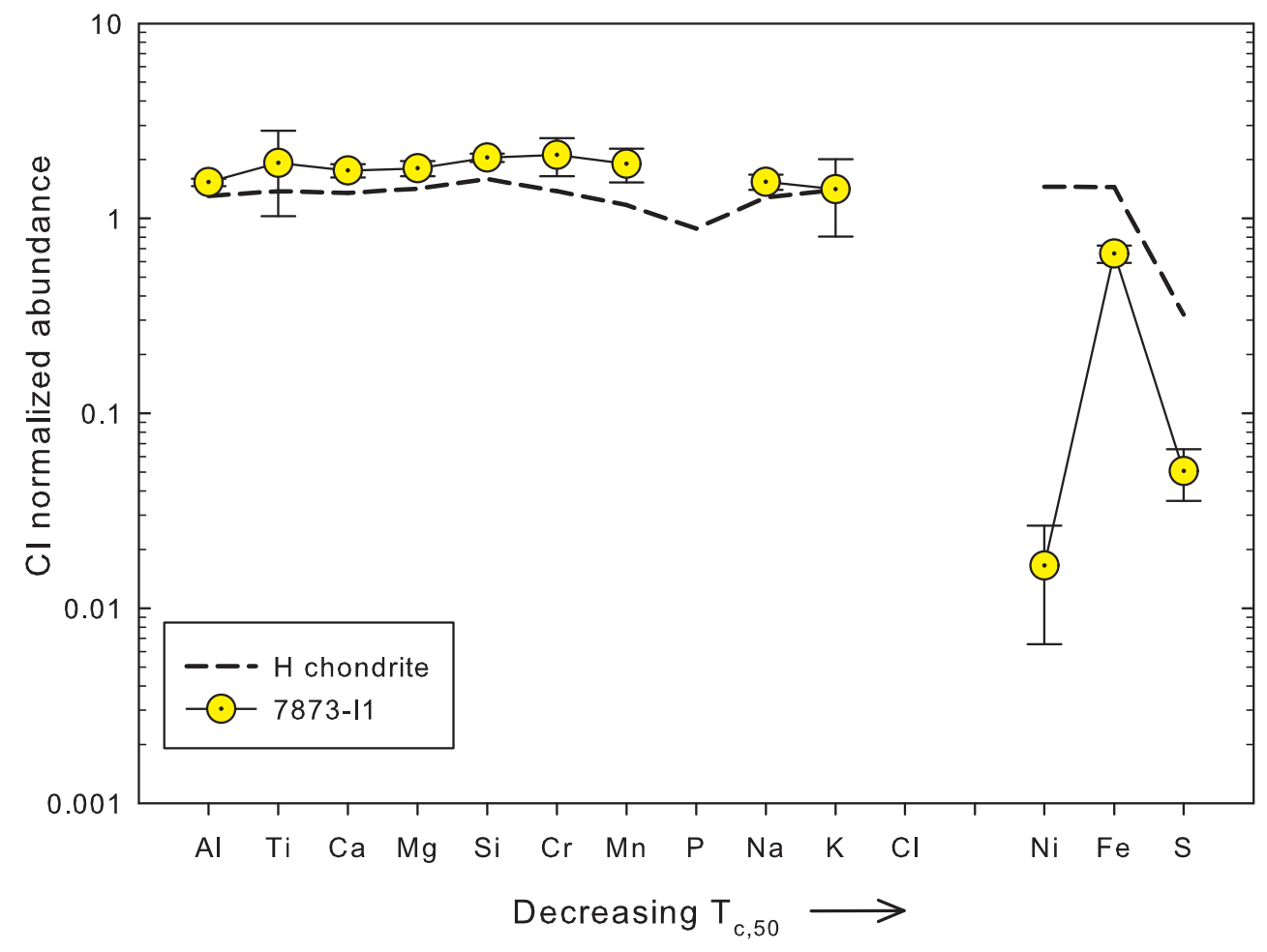

Figure A.8: CI-normalized bulk chemistry of 7873-I1. This inclusion is essentially chondritic, except for a strong metal and sulfide depletion and a complete lack of phosphates.

\section{A.4 Etter (AMNH 4462) H6}

Etter is an H6 chondrite. One inclusion from one PTS was examined. 
A Chemical and petrographic descriptions of individual inclusions

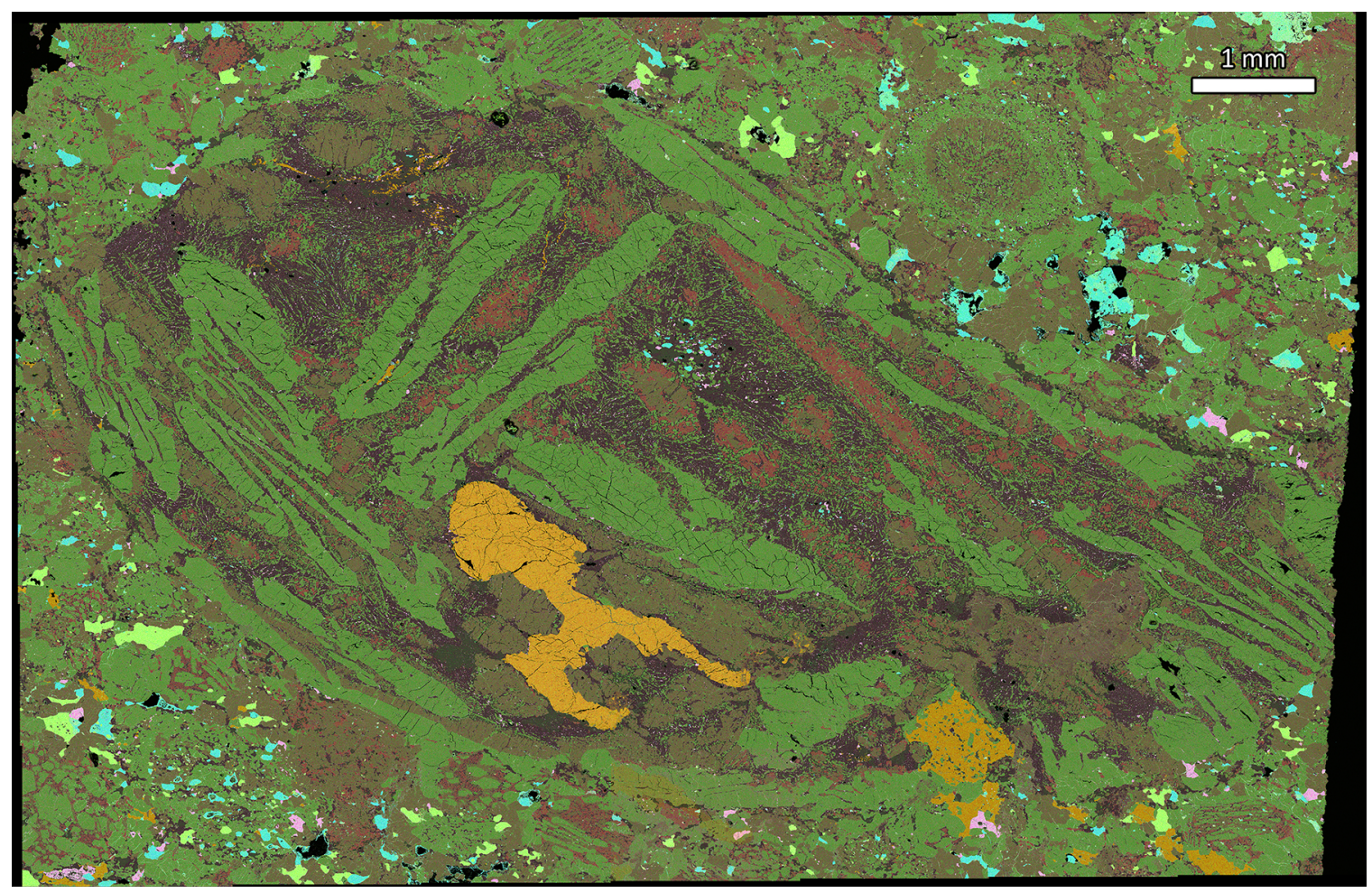

Figure A.9: False-color EDS phase map of Etr-I1. Color key same as in Tdk-I2.

Etr-I1 This inclusion is large and oval-shaped, $13 \times 6.25 \mathrm{~mm}$ in apparent size. Its contact with the host is fuzzy but readily discernible. Some crystals at one edge appear broken, suggesting this is a fragment of a larger object, however, the contact on the other side of the inclusion is, for a large fraction, dominated by a mostly unbroken olivine rim; perhaps this edge is a primary feature.

The texture can be best described as porphyritic. Phenocrsyts are coarse, skeletal olivine up to several millimeters in length as well as smaller low-Ca pyroxene rimmed with diopside. There is also one very coarse (several $\mathrm{mm}$ ) chlorapatite grain, and another, smaller, merrillite grain. One anhedral, glassy region has a pyroxene stoichiometry and a very $\mathrm{Na}-\mathrm{Al}$ rich chemistry. These large crystals are set in a matrix 
A Chemical and petrographic descriptions of individual inclusions

of fine-grained, anhedral olivine $(\sim 10-50 \mu \mathrm{m})$, minute chromite and metal blebs, and a feldspathic glass.

The olivine and low-Ca pyroxene is equilibrated, with average composition Fa 25.5 $\pm 0.3(\mathrm{~N}=17)$ and Wo $1.5 \pm 0.1$, Fs $21.5 \pm 0.3$, En $77.0 \pm 0.3(\mathrm{~N}=7)$, respectively. The diopside is also highly equilibrated, averaging Wo $44.3 \pm 0.4$, Fs $8.7 \pm 0.5$, En $46.9 \pm 0.3(\mathrm{~N}=10)$. There are two distinct feldspathic phases, one crystalline with composition $\mathrm{Ab} 82.7 \pm 2.5$, Or $1.0 \pm 0.1$, An $16.2 \pm 2.5(\mathrm{~N}=8)$, and another glassy, with composition Ab $78.3 \pm 1.2$, Or $19.5 \pm 2.8$, An $2.2 \pm 1.6(\mathrm{~N}=6)$. Olivine, low-Ca pyroxene, diopside, both feldspars, chromite, and metal were determined with EDS point spectra. Both phosphates and sulfide were determined with AZtec's analyze phases. 
A Chemical and petrographic descriptions of individual inclusions

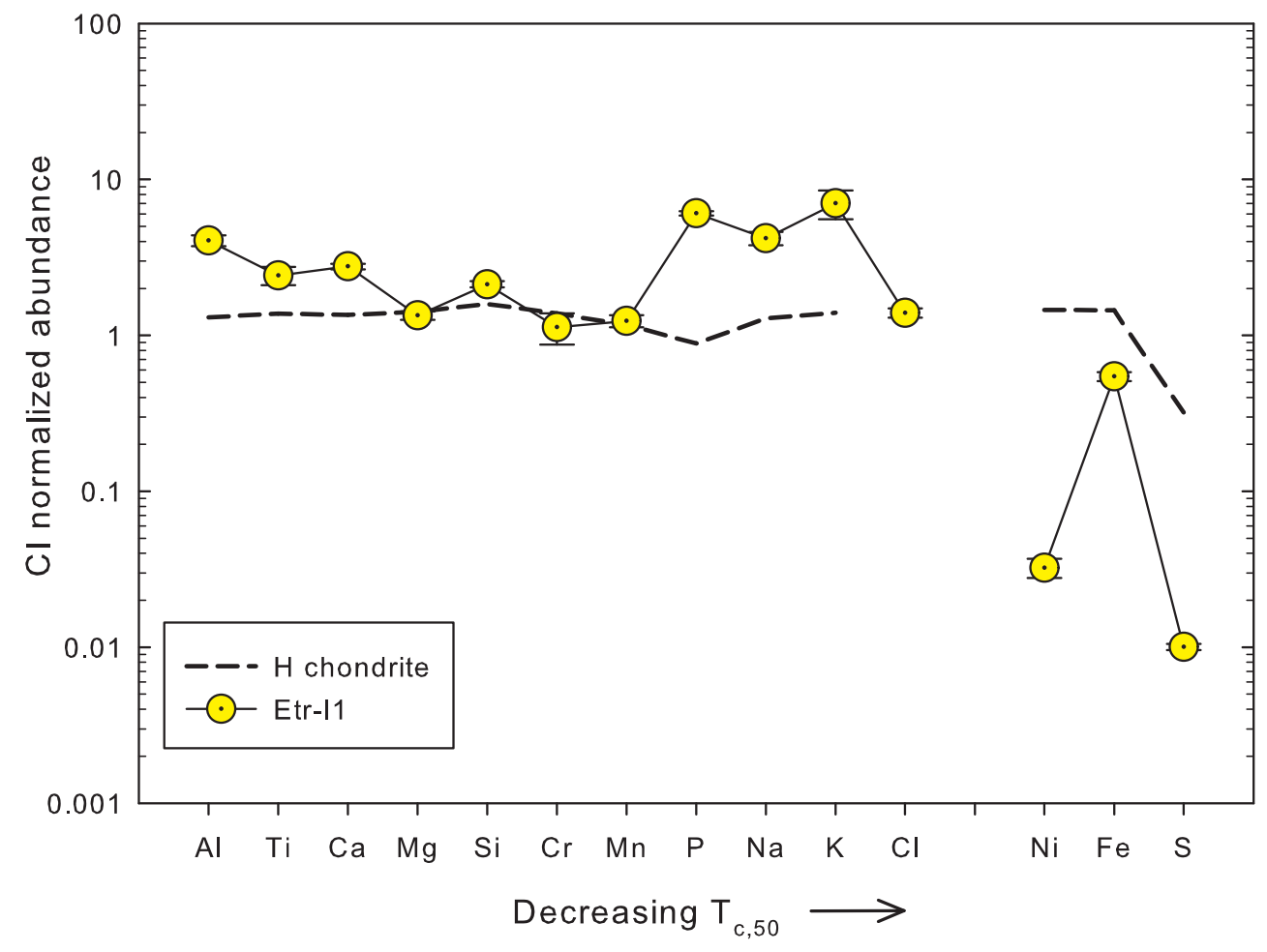

Figure A.10: CI-normalized bulk chemistry of Etr-I1. Notable are an enrichment in phosphorous, due to two very coarse phosphate grains, and enrichments in alkali elements, aluminum, and calcium. The enrichment pattern could be explained by volatility fractionation followed by metasomatism, but is more readily explained by an enrichment in feldspar, which is also supported by high normative plagioclase content (see figure 3.4 and section 4.3.2). 


\section{A.5 Gunlock (AMNH 4620) L3.6}

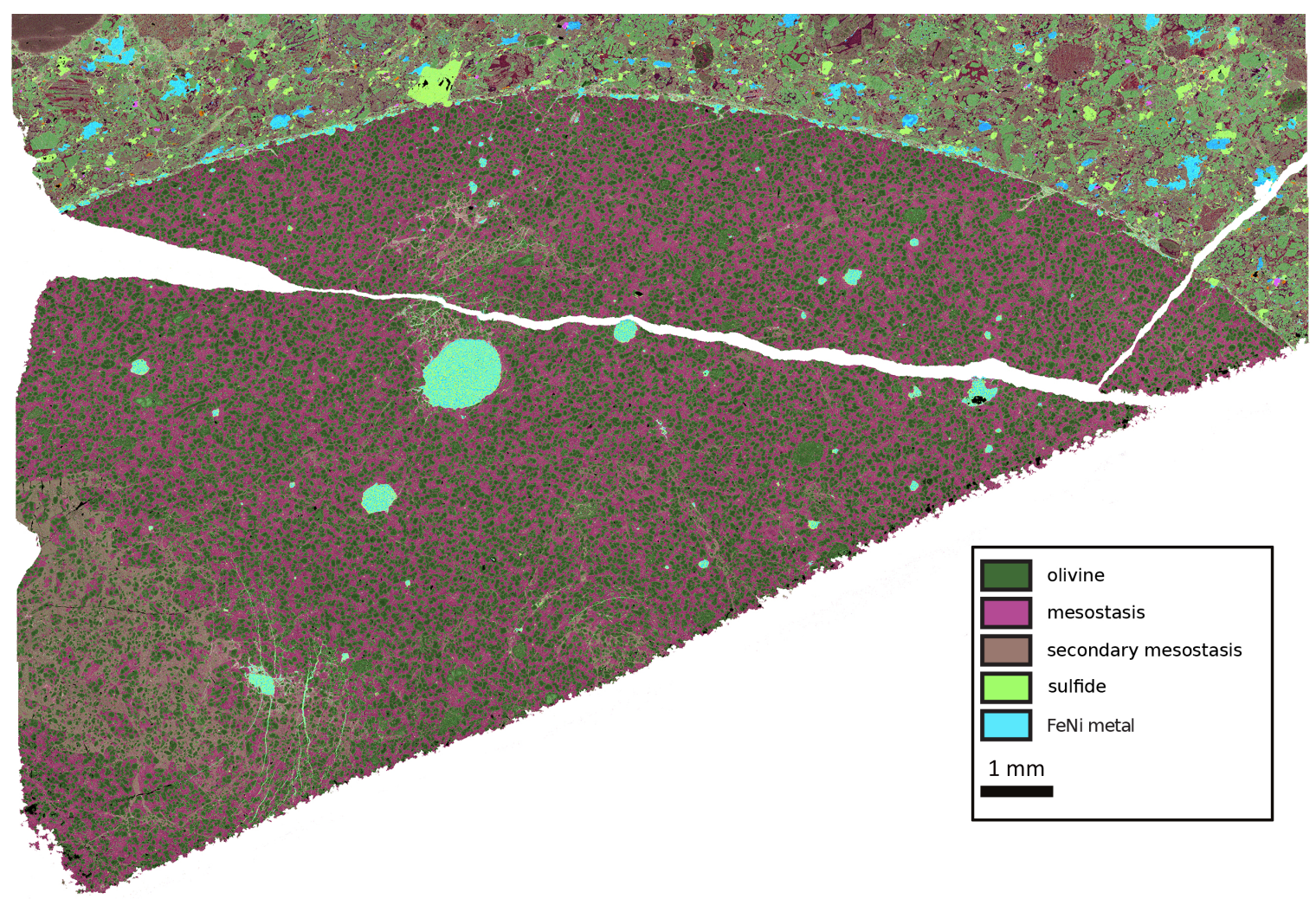

Figure A.11: False-color EDS phase map of Glk-I1

Glk-I1 Two thin sections of the same inclusion have been examined, and appear essentially identical. The inclusion is exceptionally large, as previously reported by Prinz et al. (1988), who estimated the original diameter of the object to be $5 \mathrm{~cm}$, based on the curved contact with the host. The inclusion is an olivine microporphyry, similar in texture to the inclusion in NWA 869. Small, subhedral to euhedral olivine phenocrysts with an average size of $50 \mu \mathrm{m}$ are in a glassy mesostasis. The majority are zoned, with magnesian cores and ferroan rims. A small fraction of the olivine phenocrysts, however, are larger $(200-300 \mu \mathrm{m})$, and appear to be agglomerations of 
A Chemical and petrographic descriptions of individual inclusions

smaller grains. These phenocrysts have a core of equilibrated olivine and are mantled by smaller, zoned olivine crystals.

Other, rare large olivine phenocrysts have a reversed zoning pattern, with ferroan cores and magnesian rims, and at least one has been found with multiple zonation bands. These crystals contain sulfide within cracks, and while they are overall euhedral, their edges are broken and eroded, unlike the majority of the phenocrysts, which have distinct and well-formed edges.

Coarse $(100-1000 \mu \mathrm{m})$, round metal and sulfide intergrowths are throughout the inclusion, and the same metal and sulfide mixture forms a thin rim at the host contact.

The inclusion is crossed by multiple fractures, along which olivine phenocrysts are truncated and offset, though the fractures do not extend into the host. Additionally, two regions in the inclusion have been significantly brecciated, with clasts of the inclusion material and individual olivine phenocrysts suspended in a mesostasis that is chemically different (see figure) Where this occurs near the metal and sulfide grains, metal and sulfide has mobilized and filled fractures.

The olivine has average composition of Fa $11.4 \pm 2.3(\mathrm{~N}=21)$, which is low for an L chondrite. The majority of the mesostasis (pink in the images) is feldspathic compared to the secondary mesostasis (brown in the images), which is considerably more magnesian. All phase compositions were determined via EDS map extracts. 
A Chemical and petrographic descriptions of individual inclusions

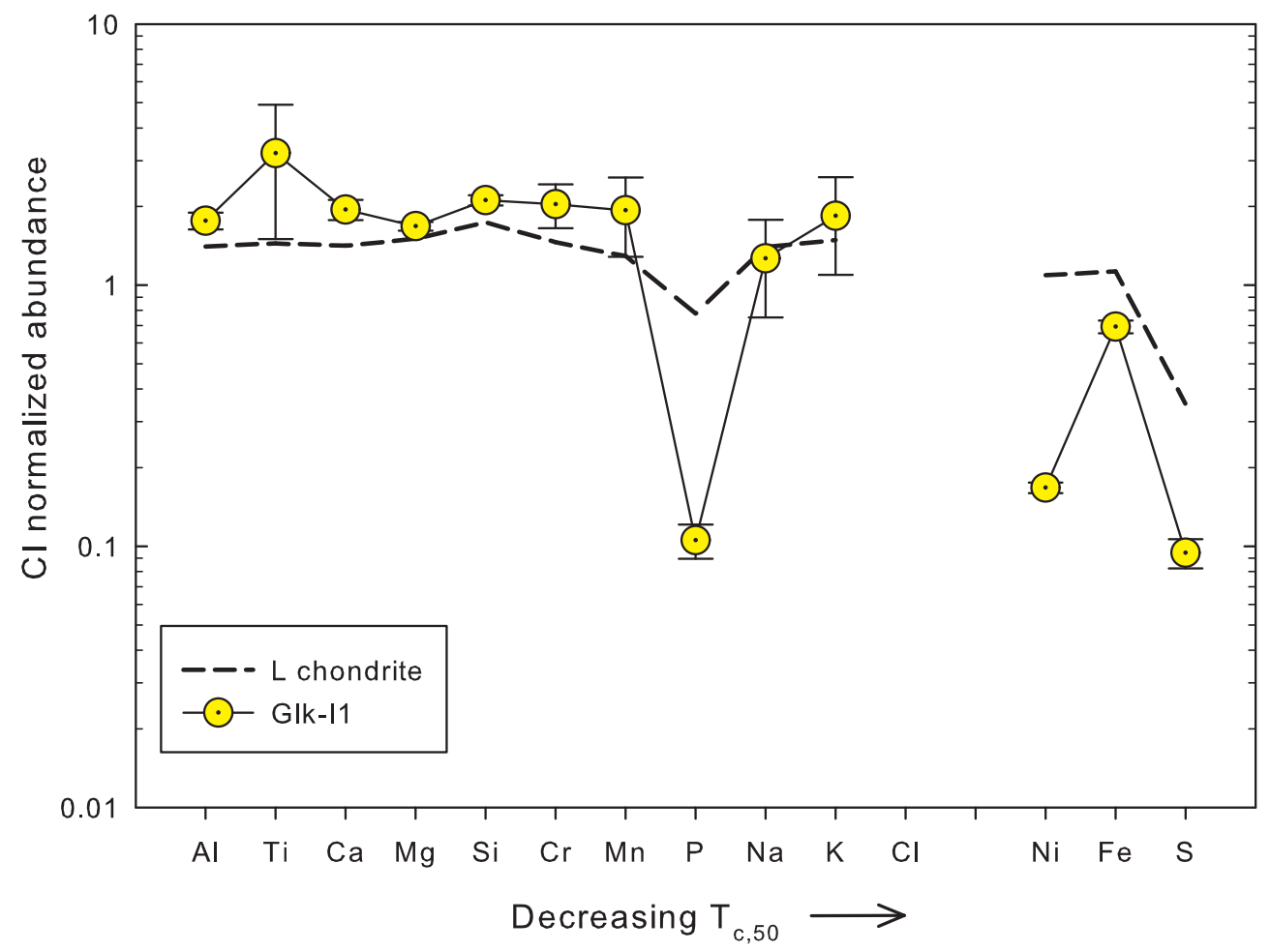

Figure A.12: CI-normalized bulk chemistry of Glk-I1. Except for depletions in phosphate, sulfide and metal minerals this inclusion is essentially chondritic.

\section{A.6 Khohar (AMNH 4245) L3.6}

Khohar is an L3.6 chondrite. One thin section with one inclusion has been examined. 
A Chemical and petrographic descriptions of individual inclusions

\section{A.6.1 Khr-I1}

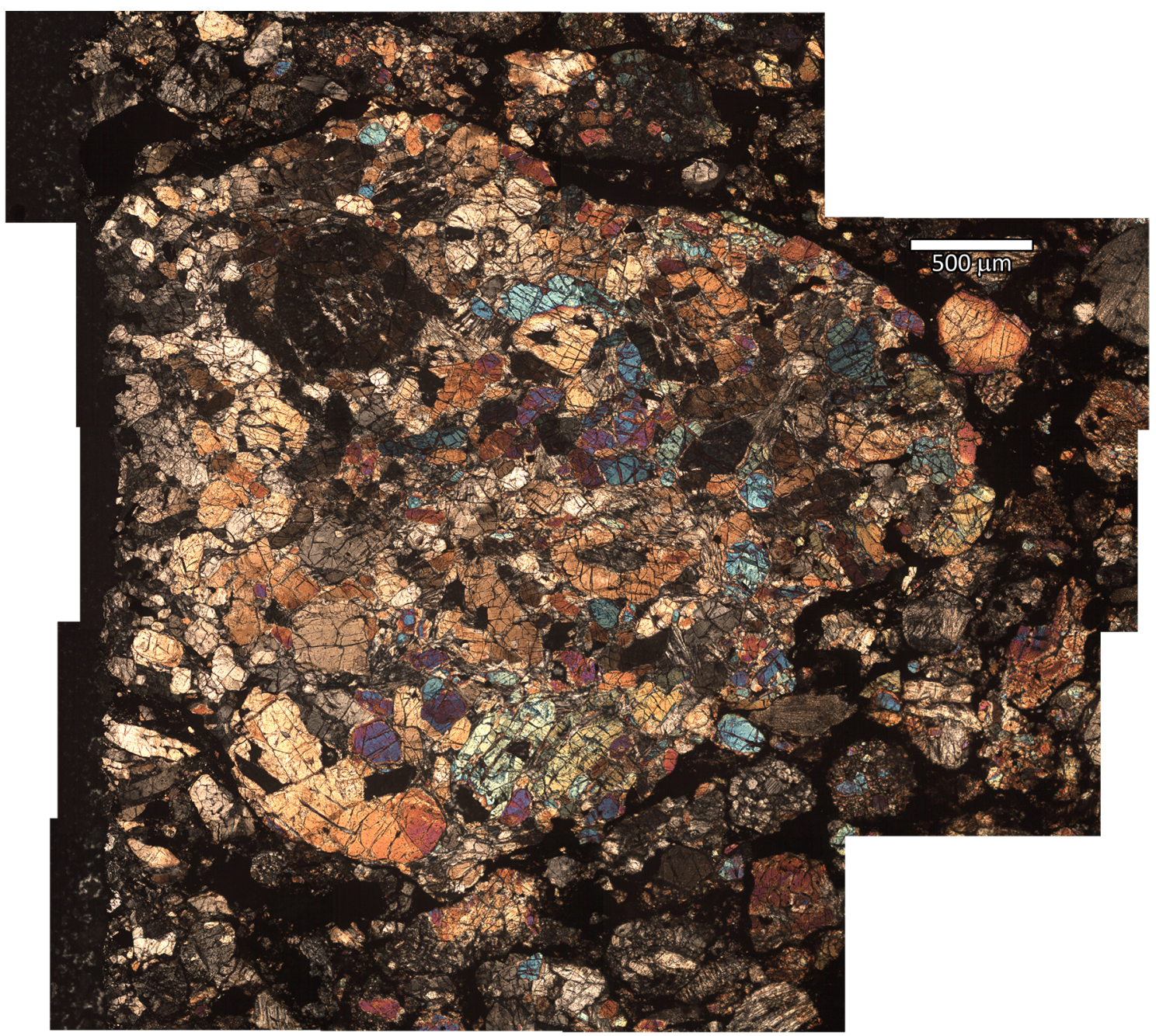

Figure A.13: Khr-I1 under cross-polarized light.

The inclusion is oblong and subrounded, with an apparent diameter of $\sim 3.5 \mathrm{~mm}$, though one edge is missing from this specimen. The contact with the host is defined by a thin $(10-50 \mu \mathrm{m})$ shock vein. Some olivine crystals protrude from the edge, and others have clearly been broken, suggesting that this inclusion is a fragment of a 
A Chemical and petrographic descriptions of individual inclusions

larger object.

The inclusion is an olivine microporphyry, with unequilibrated, subhedral to anhedral olivine phenocrysts with intergrown anhedral pigeonite and feldspar interstitial. Olivine phenocrysts range in size from $\sim 50 \mu \mathrm{m}$ to $\sim 300 \mu \mathrm{m}$. Minor troilite, kamacite, taenite, and chromite exist as $\sim 10-100 \mu \mathrm{m}$ blebs, or euhedral to subhedral crystals for the chromite.

Most of the olivine is equilibrated, but there are a few grains that have a variable composition, resulting in an average Fa of $19.2 \pm 2.0(\mathrm{~N}=22)$. The pigeonite is also somewhat unequilibrated, with Wo $12.5 \pm 1.7$, Fs $21.1 \pm 1.3$, En $66.4 \pm 1.6(\mathrm{~N}=$ 14). The feldspar is predominantly very Ca-rich, but some crystals contain albiteanorthite exsolution, so the average composition is highly variable, with Ab $22.0 \pm$ 6.8, Or 1.6 \pm 0.6 , An $77.4 \pm 6.9(\mathrm{~N}=12)$. All phases were determined through both EDS point spectra and EDS map extracts.

There are at least two, and possibly three, olivine "donuts" present in this inclusion (see section 4.4), where a ring and some interior bars of olivine share an optical orientation, suggesting they grew as one crystal. These objects are 600, 500, and 375 $\mu \mathrm{m}$ in apparent diameter. 
A Chemical and petrographic descriptions of individual inclusions

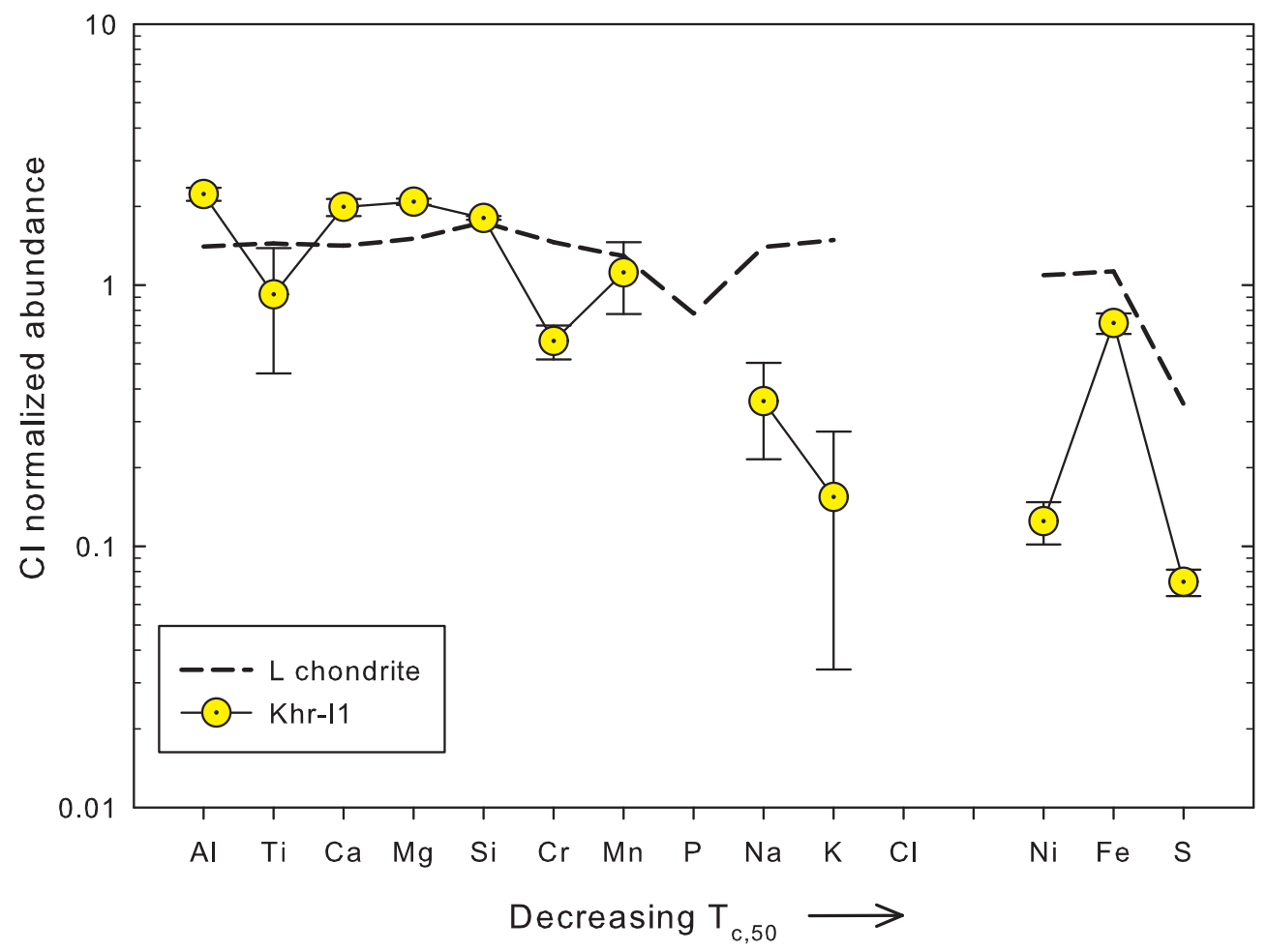

Figure A.14: CI-normalized bulk chemistry of Khr-I1. This inclusion did not contain any detectable phosphate minerals (see section 4.3.5). It is also depleted in volatile elements, suggesting a vapor fractionation process.

\section{A.7 NWA 7869 (CML 0143) L3.7}

The meteorite NWA 7869 is an L3.7 chondrite. Two unique inclusions, approximately $1.5 \mathrm{~mm}$ apart from each other in one polished thin section, were examined. 
A Chemical and petrographic descriptions of individual inclusions

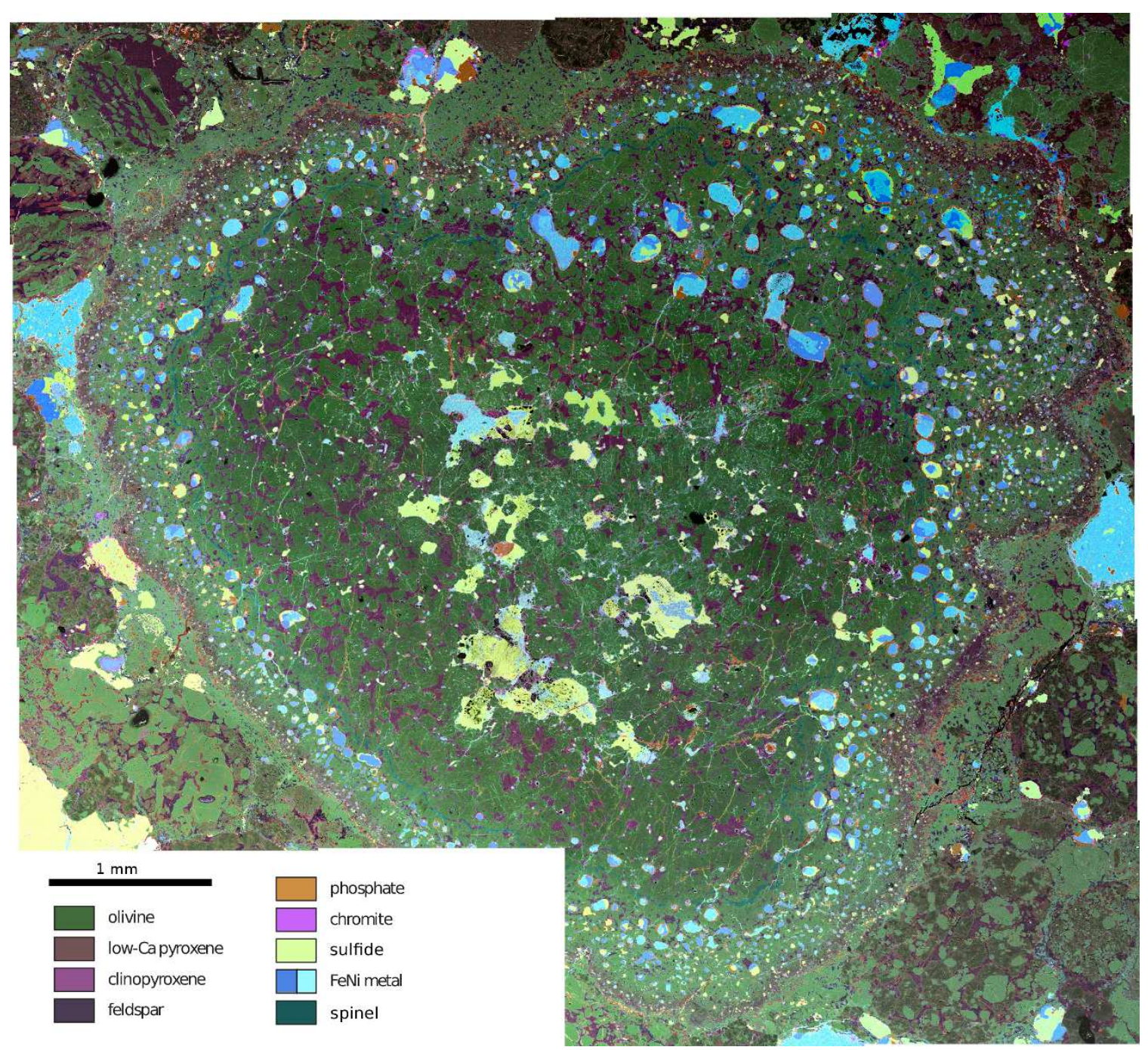

Figure A.15: False-color chemical map of inclusion in NWA 7869-1.

NWA 7869-I1 The larger of the two, here called NWA 7869-1 is rounded with an apparent diameter of approximately $5 \mathrm{~mm}$. The inclusion has a well defined edge and a radial variation in texture, which suggest it formed as a free-floating droplet.

The silicate phase is predominately fine-grained subhedral to anhedral olivine, with an average grain size of $70 \mu \mathrm{m}$, with a range of $25-100 \mu \mathrm{m}$. Low-Ca pyroxene is 
A Chemical and petrographic descriptions of individual inclusions

common at the host contact, forming a thin $(\sim 100 \mu \mathrm{m})$ rim around the inclusion, but is absent in the interior. Small, bladed, euhedral to subhedral feldspar and high-Ca pyroxene $(12 \times 3 \mu \mathrm{m})$ crystals are interstitial to the olivine grains throughout the inclusion. These crystals are larger on average in the center of the inclusion $(\sim 50 \mathrm{x}$ $5 \mu \mathrm{m}$ for feldspar, $\sim 12 \times 3 \mu \mathrm{m}$ for pyroxene) and smaller towards the inclusion edge (20 x $2 \mu \mathrm{m}$ for feldspar, $10 \times 1 \mu \mathrm{m}$ for pyroxene).

The olivine is unequilibrated, with average $\mathrm{Fa}$ of $19.3 \pm 4.8(\mathrm{~N}=27)$, but the variation in $\mathrm{Fa}$ is radial, with more forsteritic olivine in the center of the inclusion $($ Fa $14.7 \pm 1.5, \mathrm{~N}=12)$ and more fayalitic at the edges $(\mathrm{Fa} 23.0 \pm 2.8, \mathrm{~N}=15)$. Radial EMPA points indicate a smooth gradient between the lowest values (Fa 14) at the center to the highest (Fa 23) at the edge. Some of the outer olivine grains are zoned, with magnesian cores close to the composition of the olivine at the interior of the inclusion.

The low-Ca pyroxene composition varies between Fs 7, En 88 and Fs 24, En 73, $(\mathrm{N}=9)$ and the calcic pyroxene is a diopside, with Wo $50.6 \pm 0.7$, Fs $1.0 \pm 0.3$, En $48.4 \pm 2.3(\mathrm{~N}=9)$, and no significant variation. Average feldspar composition is $\mathrm{Ab}$ $28.9 \pm 5.3$, An $70.5 \pm 4.9(\mathrm{~N}=11)$, and is also radially dependent, though only at the edge of the inclusion very close to the contact with the host. Here the feldspar is more sodic $(\mathrm{Ab} \sim 30)$ than the majority of the inclusion, where it is largely uniform $(\mathrm{Ab} \sim 25)$.

Spinel is present as a thin $(\sim 10 \mu \mathrm{m})$ band about $2 / 3$ of the distance between the center and the edge of the inclusion. Merrillite phosphate is present as rims around many of the metal and sulfide blebs, and also as a thin $(10 \mu \mathrm{m})$ band at the inclusion-host contact, outside of the low-Ca pyroxene rim. Chromite is a minor 
A Chemical and petrographic descriptions of individual inclusions

phase, accounting for only $0.2 \pm 0.02 \mathrm{vol} \%$ of the inclusion, and it occurs in such small crystals that a reliable chemical analysis was not possible, so it was assumed to be pure $\mathrm{FeCr}_{2} \mathrm{O}_{4}$.

Olivine composition was determined with EMPA and EDS point spectra. Both pyroxenes and feldspar were determined with EDS point spectra. Phosphate, chromite, metal, sulfide, and spinel were determined with AZtec's analyze phases.

This inclusion is anomalous in that it is not depleted in metal and sulfide (see figure A.15). It contains some amorphous, weathered metal in the center and the outer $\sim 500 \mu \mathrm{m}$ of the inclusion contains metal and sulfide globules that average 50 $\mu \mathrm{m}$ in diameter, but have a wide size distribution $(10 \mu \mathrm{m}-150 \mu \mathrm{m})$. Metal accounts for approximately 7 volume $\%$ of the inclusion and is about $70 \%$ kamacite (5 atom \% $\mathrm{Ni}$ ) and $30 \%$ Taenite (40 atom \% Ni). The metal in the center is entirely kamacite, while the blebs at the edge of the inclusion are either kamacite, taenite, or both. 
A Chemical and petrographic descriptions of individual inclusions

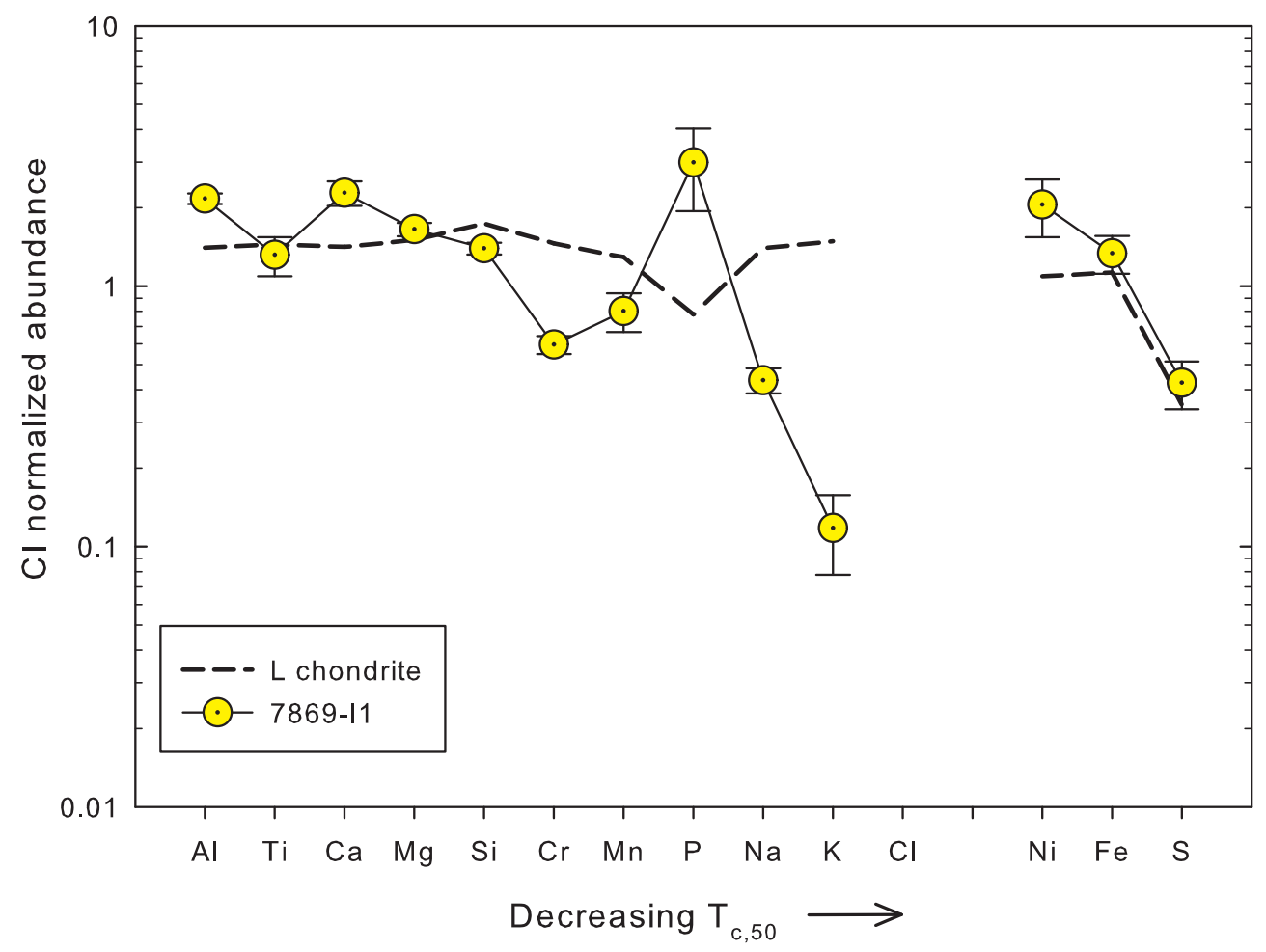

Figure A.16: CI-normalized bulk chemistry of 7869-I1. This is the only inclusion in this study not depleted in metal and sulfide. There is also a notable enrichment in phosphorous (see section 4.3.5). Otherwise this inclusion is depleted in volatile elements, suggesting a volatility fractionation process. 
A Chemical and petrographic descriptions of individual inclusions

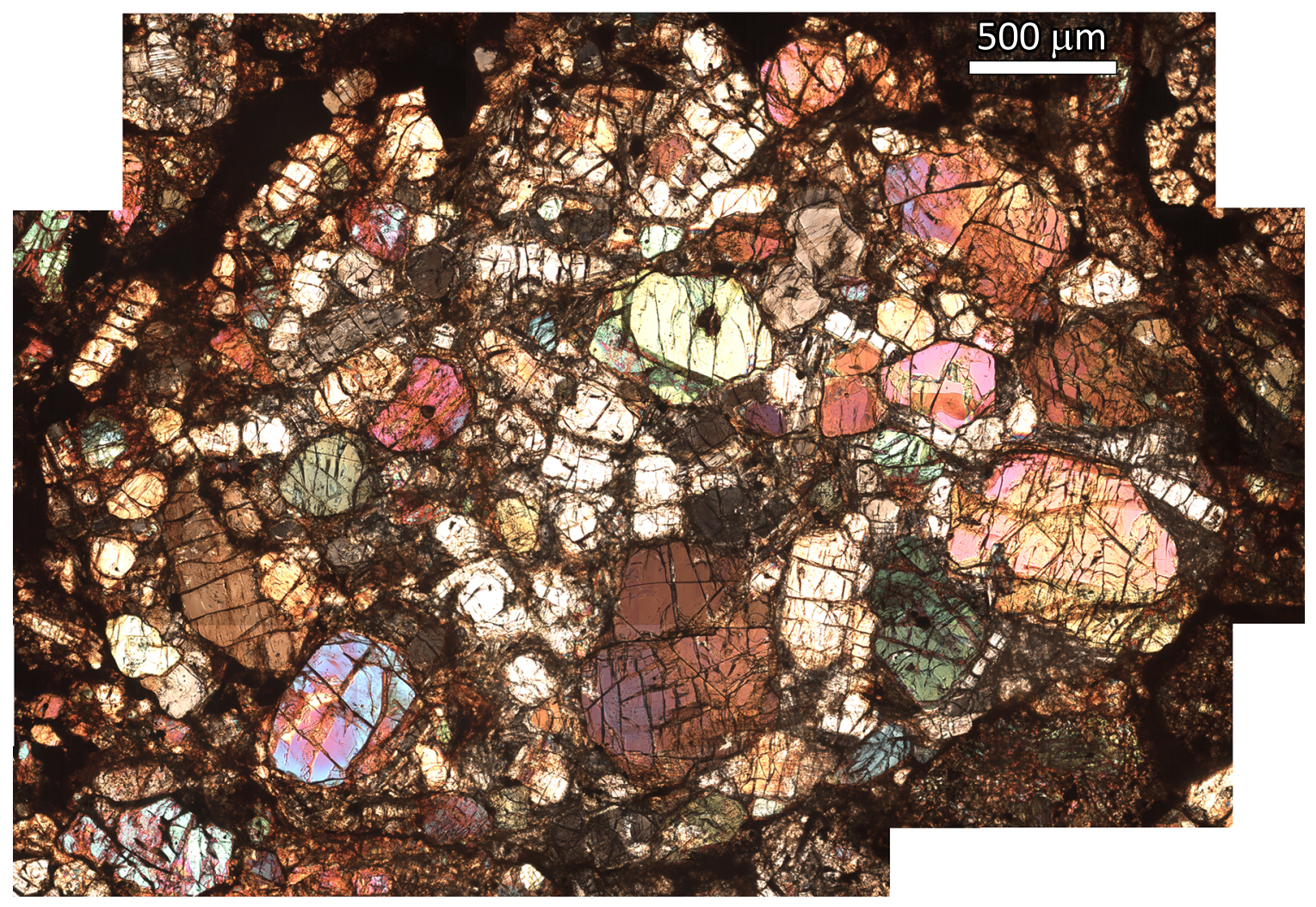

Figure A.17: Smaller inclusion in NWA 7869 viewed with cross-polarized light.

NWA 7869-I2 The second inclusion, NWA 7869-2, is smaller and oval-shaped, with a $3.5 \mathrm{~mm}$ mean apparent diameter (see figure A.17). It has larger, subhedral grains overall, as well as a much wider range of grain sizes, from $10-700 \mu \mathrm{m}$. Some of the grains at the margin appear broken, and some large grains protrude, giving the impression of having been brecciated and subsequently eroded. With this and no radial variation in texture, it appears that this is a clast from a larger object, though one that has experienced some abrasion.

The mineralogy is an almost equal mix of olivine (36 volume \%) and low-Ca pyroxene (34 volume \%), with interstitial feldspar and high-Ca pyroxene. The olivine 
A Chemical and petrographic descriptions of individual inclusions

is unequilibrated and the larger grains are zoned, with magnesian cores (Fa $19.7 \pm$ 1.6, $\mathrm{N}=7$ ) and ferroan rims (Fa $25.6 \pm 1.7, \mathrm{~N}=13$ ), but there is no radial variation in composition within the inclusion. The high-Ca pyroxene (Wo $28.5 \pm 5.8$, Fs 17.9 \pm 5.0 , En $53.6 \pm 1.8, \mathrm{~N}=14$ ) forms both singular grains, as well as overgrowths on the low-Ca pyroxene (Wo $1.2 \pm 0.6$, Fs $15.9 \pm 2.0$, En $82.9 \pm 2.6, \mathrm{~N}=10$ ).

The inclusion is almost void of metal and sulfide The metal and sulfide occurs primarily as large $(100 \mu \mathrm{m})$ grains on the edge of the inclusion, though there are also minute $(\sim 20 \mu \mathrm{m})$ metal, sulfide, and chromite grains throughout the inclusion. The metal that is there is kamacite. Olivine and low-Ca pyroxene were determined with both EMPA and EDS point spectra. High-Ca pyroxene and feldspar were determined with EDS point spectra, while chromite and metal were determined with AZtec's analyze phases. 
A Chemical and petrographic descriptions of individual inclusions

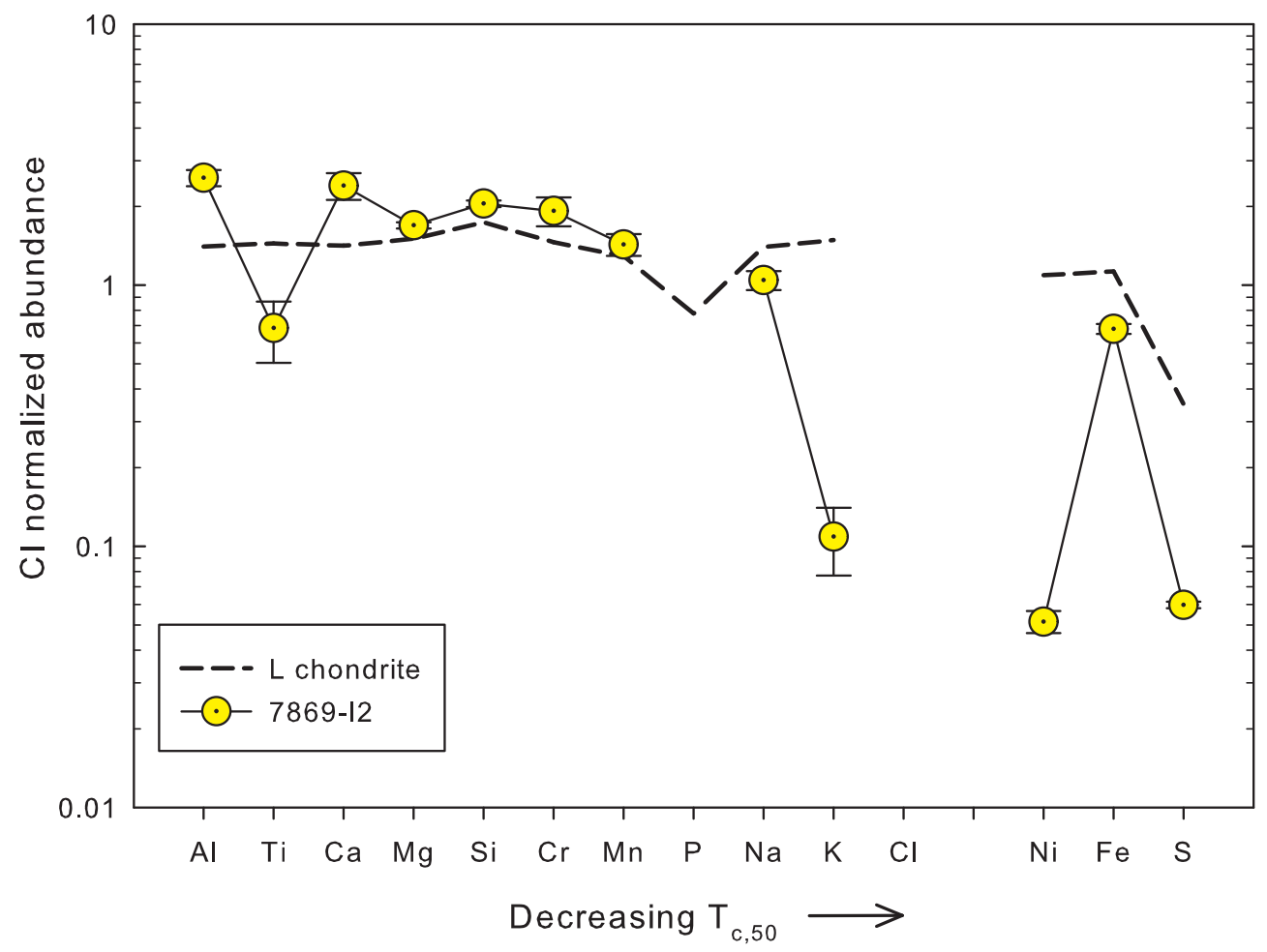

Figure A.18: CI-normalized bulk chemistry of 7869-I2. Aside from Ti, this inclusion shows a progressive depletion of volatile elements, suggesting a vapor fractionation process.

\section{A.8 NWA 7872 (CML 0359) L3.7}

NWA 7872 is an L4 chondrite. One PTS containing one inclusion has been examined. 
A Chemical and petrographic descriptions of individual inclusions

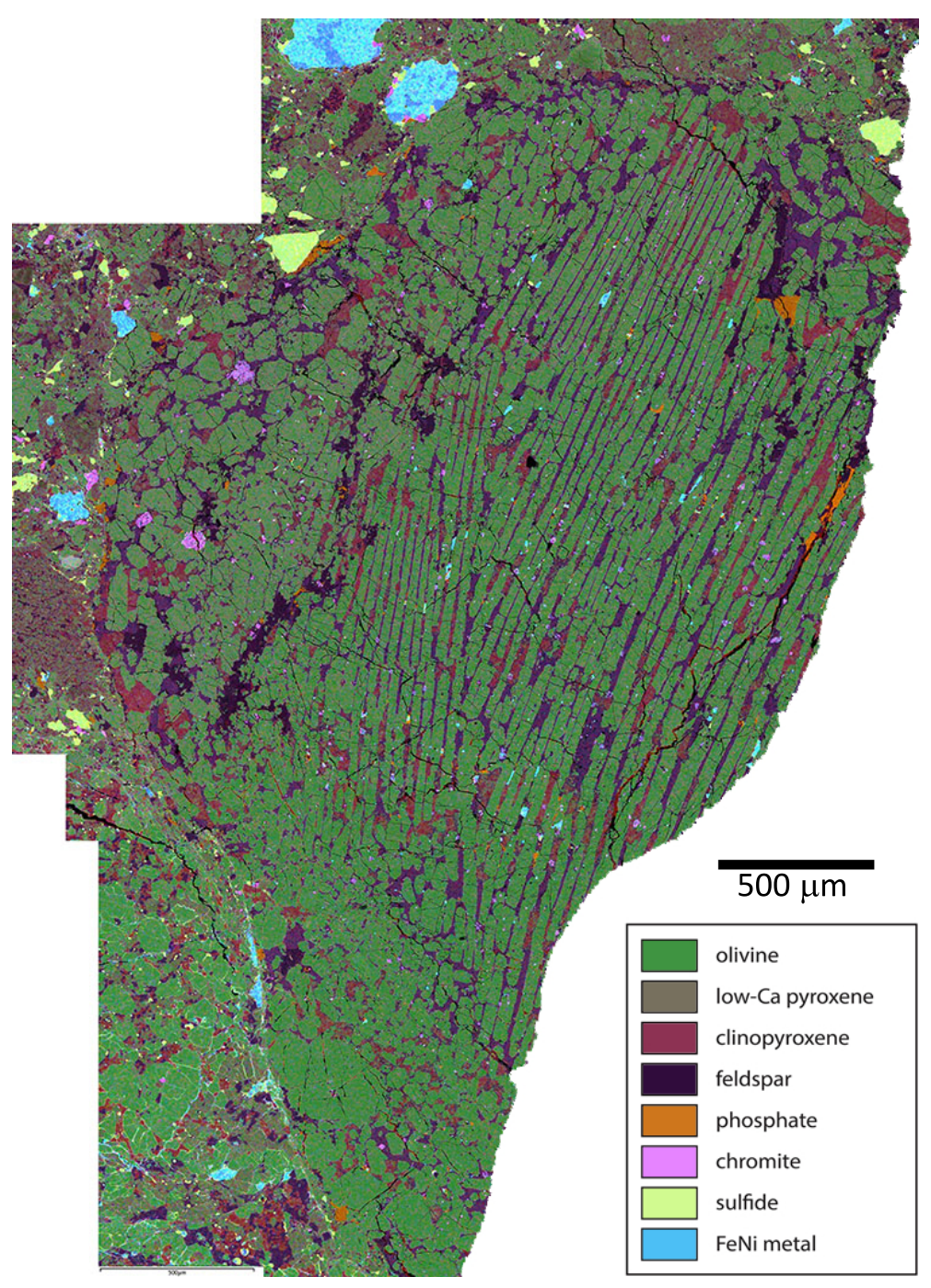

Figure A.19: False-color chemical map of NWA 7872-I1

NWA 7872-I1 Texturally, this inclusion resembles the inclusion in NWA 7870 described below, though it is broken at the edge of the sample. It is in sharp, curved contact with the host, and based on this curvature is estimated to have had an original apparent diameter of $\geq 4 \mathrm{~mm}$. Crystals at the contact are mostly unbroken. A region of shock-darkened material is nearby and in contact with the inclusion. 
A Chemical and petrographic descriptions of individual inclusions

As in NWA 7870-I1, the texture is dominated by skeletal olivine that appears concentric about the original center of the inclusion, which transitions to subhedral granular olivine at the host contact. The skeletal olivine crystals are quite coarse, averaging $1 \mathrm{~mm}$ in length and $30 \mu \mathrm{m}$ in width. The granular olivine averages 200 $\mu \mathrm{m}$ in diameter. Unlike NWA 7870-I1, this inclusion does not contain a significant amount of low-Ca pyroxene. Interstitial to the olivine is anhedral calcic pyroxene of variable composition, with both diopside and pigeonite present, anhedral Ca-feldspar and anhedral Na-feldspar. Merrillite and chlorapatite are present as grains of variable sizes, from $\sim 10-60 \mu \mathrm{m}$. Small $(\sim 10 \mu \mathrm{m})$ blebs of FeNi metal, troilite, and chromite are throughout the inclusion, as are minute $(<10 \mu \mathrm{m})$ blebs of ilmenite.

The olivine is highly equilibrated, with average $\mathrm{Fa}=24.4 \pm 0.3(\mathrm{~N}=16)$, consistent with an L chondrite. The inclusion's host meteorite is unequilibrated, however, so the inclusion must have been thermally processed before it became incorporated into the host. As mentioned, Ca-rich pyroxene is variable in composition, with average and range of Wo $38.7 \pm 6.6$, Fs $11.0 \pm 2.1$, En $50.1 \pm 4.7(\mathrm{~N}=11)$. The anorthositic feldspar has composition Ab $13.9 \pm 4.2$, Or $0.3 \pm 0.2$, An $85.9 \pm 4.3(\mathrm{~N}$ $=13$ ), and the albitic feldspar has composition Ab $86.6 \pm 1.8$, Or $5.6 \pm 1.5$, An $7.4 \pm$ $0.7(\mathrm{~N}=5)$. Olivine and the more anorthitic feldspar compositions were determined with EMPA and EDS point spectra. Diopside and the more albitic feldspar were determined with EDS point spectra and EDS map extracts. Phosphate, metal, and chromite were determined with EDS point spectra and AZtec analyze phases. 
A Chemical and petrographic descriptions of individual inclusions

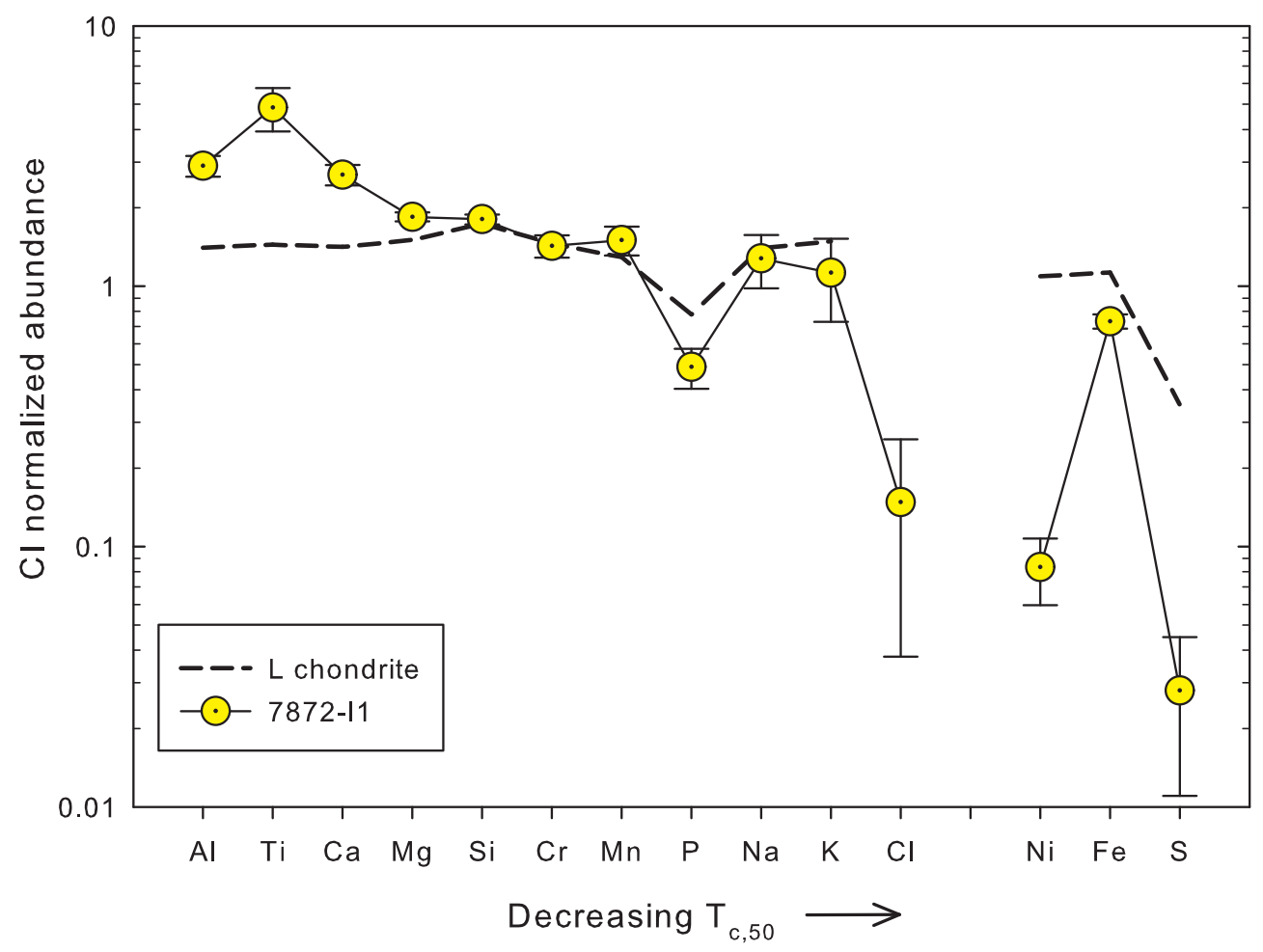

Figure A.20: CI-normalized bulk chemistry of 7872-I1. This inclusion is enriched in refractory elements, and subtly depleted in volatile elements, suggesting a vapor fractionation process.

\section{A.9 NWA 869 (CML 0074) L3-6}

NWA 869 is an L3-6 breccia. One PTS containing one inclusion has been examined. 
A Chemical and petrographic descriptions of individual inclusions

\section{A.9.1 NWA 869-I1}

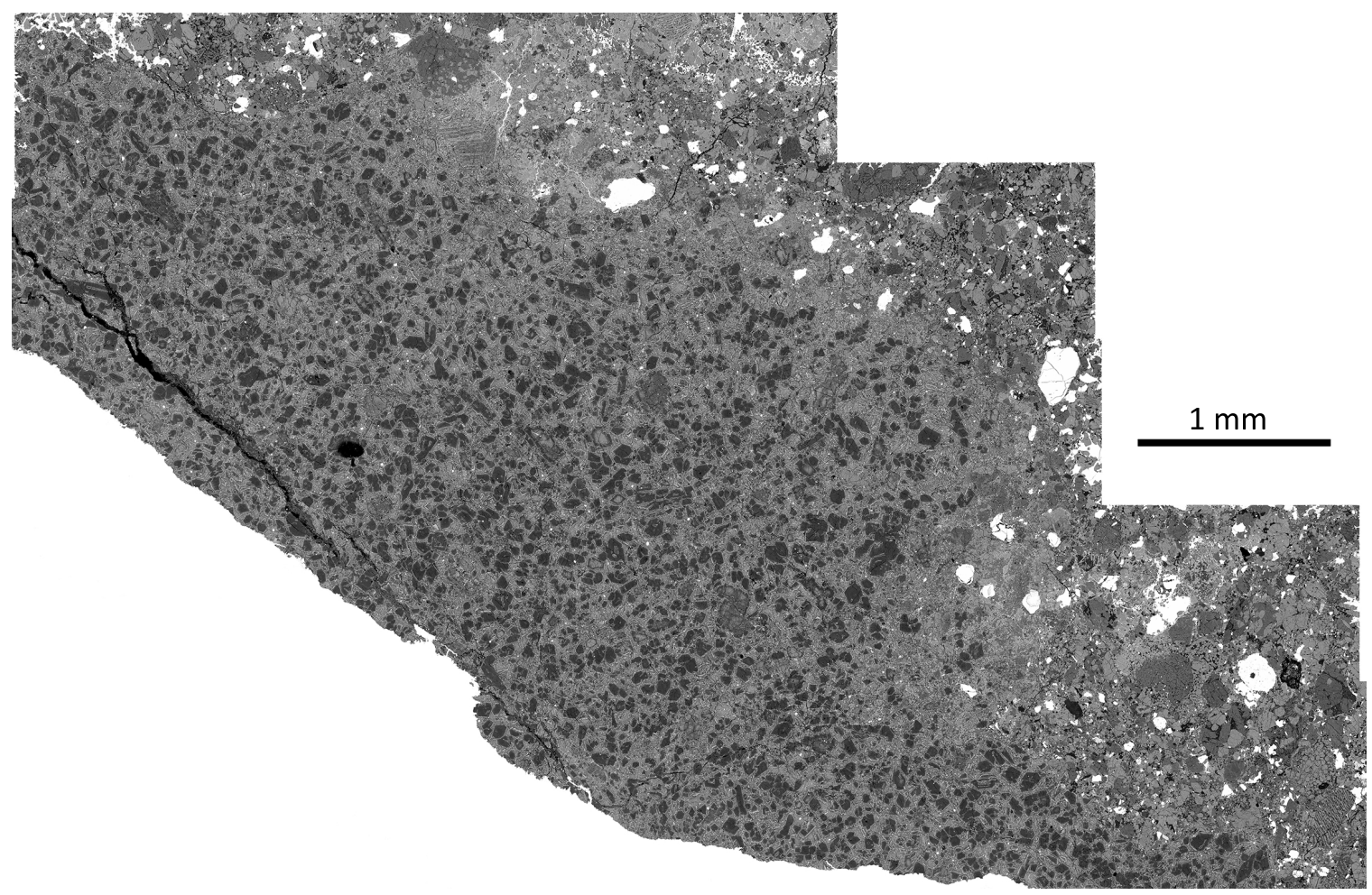

Figure A.21: Backscattered electron micrograph of 869-I1.

The inclusion is an olivine microporphyry, with euhedral to subhedral olivine phenocrysts set in a glassy mesostasis. This inclusion is on the edge of the sample, and as such is difficult to estimate an original size, but based on curvature could have been several centimeters in diameter. Its contact with the host is angular and irregular. In some regions, it is sharp, with crystals in both inclusion and host broken; in others, the contact is more gradual, with the inclusion melt appearing to engulf the host material. Close to the inclusion, the host is shock darkened.

The olivine phenocrysts have a relatively small size distribution, with average 
A Chemical and petrographic descriptions of individual inclusions

grain size $\sim 100 \mu \mathrm{m}$, and a range of $50-300 \mu \mathrm{m}$. Most of the phenocrysts are zoned, with magnesian cores and narrow ferroan rims, though the larger grains have a ferroan core, suggesting that these are relict grains. Many of these larger grains also contain small sulfide blebs. About $15 \%$ of the phenocrysts are low-Ca pyroxene, most of which is in close proximity to the host contact. Troilite blebs averaging $\sim 50$ $\mu \mathrm{m}$ are throughout the inclusion, with smaller chromite and FeNi metal grains more sparsely distributed. The mesostasis is glassy, with microlites below the resolution limit.

The unequilibrated olivine has distinct chemistry; the forsteritic cores have an average composition Fa $11.4 \pm 0.9(\mathrm{~N}=20)$, with the fayalitic rims and smaller grains averaging Fa $23.1 \pm 2.8(\mathrm{~N}=15)$. As the majority of the olivine is the forsteritic cores, the average Fa value is low for an $\mathrm{L}$ chondrite. The low-Ca pyroxene is also zoned, which gives variable composition of Wo $2.3 \pm 1.1$, Fs $23.7 \pm 3.3$, En $74.0 \pm$ $3.4(\mathrm{~N}=17)$, average for an L chondrite. Olivine, low-Ca pyroxene, and mesostasis compositions were determined from EDS phase map extracts. Chromite, sulfide, and metal were determined through AZtec analyze phases.

This inclusion is cut by fractures that break and offset some of the phenocrysts, but do not extend into the host. 
A Chemical and petrographic descriptions of individual inclusions

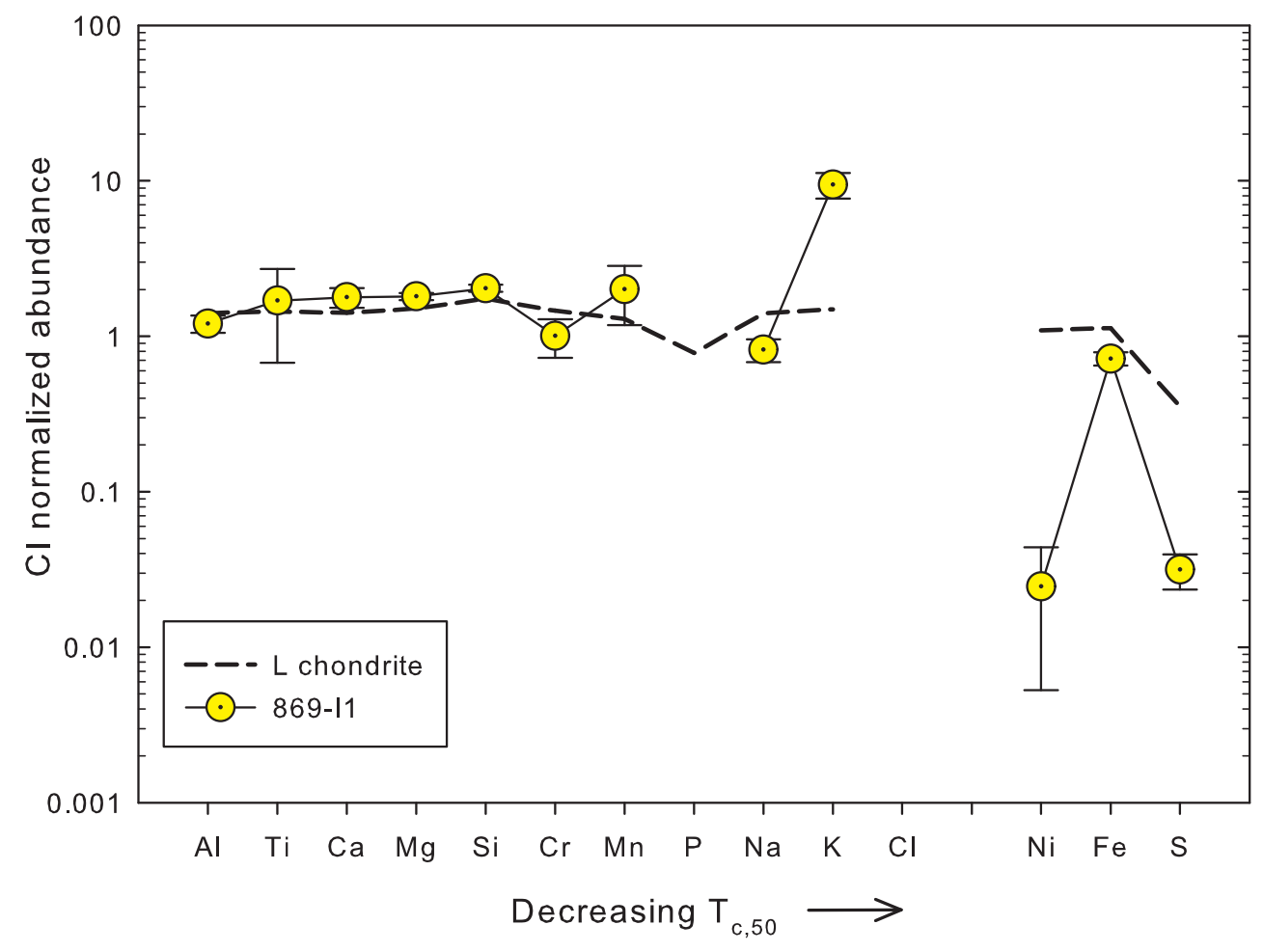

Figure A.22: CI-normalized bulk chemistry of 869-I1. This inclusion is essentially chondritic, except for a striking enrichment in potassium. This may be a signature of a shock process (see section 4.2.1).

\section{A.10 NWA 8141 (CML 0014) L3-6}

The meteorite NWA 8141 is an L chondrite breccia, with clasts of both type 3 and type 6 material. One polished thin section containing one inclusion has been examined. 
A Chemical and petrographic descriptions of individual inclusions

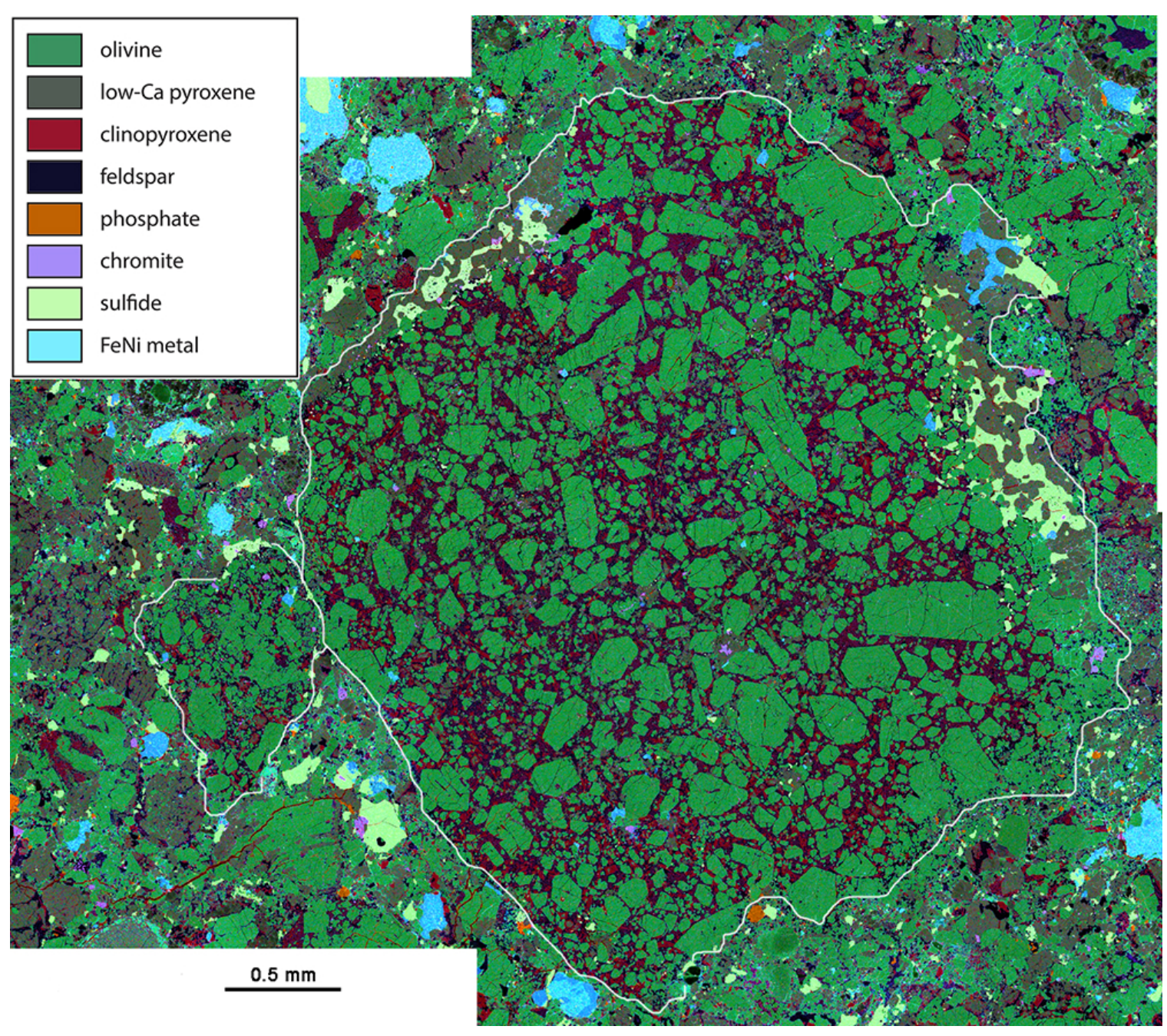

Figure A.23: False-color EDS chemical map of inclusion in NWA 8141-1.

NWA 8141-I1 The inclusion is rounded, with an apparent diameter of $3.4 \mathrm{~mm}$. It is an olivine microporphyry, with mostly euhedral olivine crystals in a groundmass of subhedral to anhedral low-Ca pyroxene, euhedral to subhedral clinopyroxene and subhedral to anhedral feldspar. Minor chromite, troilite, taenite and kamacite are present as small blebs throughout the inclusion, though some coarse troilite and metal exist at the apparent edge of the inclusion (see below). A smaller, $0.5 \mathrm{~mm}$-diameter 
A Chemical and petrographic descriptions of individual inclusions

object with similar mineralogy and texture is adjacent to the inclusion as is presumed to be a fragment of the same material (see figure A.23).

Many of the olivine phenocrysts at the inclusion-host contact are broken, indicating that the inclusion has been disrupted to some degree. Portions of the inclusion edge are mineralogically distinct, dominated by coarse-grained low-Ca pyroxene with interstitial troilite and some metal. Much of this troilite contains minute silicate inclusions.

Olivine grains average $250 \mu \mathrm{m}$ in diameter with a range of $10-680 \mu \mathrm{m}$. The other major phases have a smaller grain size distribution, averaging $\sim 25 \mu \mathrm{m}$, with the exception of the coarse low-Ca pyroxene at the edges, which are up to $200 \mu \mathrm{m}$.

The olivine in the inclusion is equilibrated, while the host is a breccia with at least one clast of unequilibrated type 3 material, indicating that the inclusion was probably thermally processed before incorporation into the host.

Olivine is Fa $24.5 \pm 0.4(\mathrm{~N}=42)$; low-Ca pyroxene is Wo $1.4 \pm 0.2$, Fs 20.6 \pm 0.8 , En $78.0 \pm 0.9(\mathrm{~N}=24)$, both of which are consistent with an $\mathrm{L}$ chondrite. Clinopyroxene is far less equilibrated with Wo $43.1 \pm 7.8$, Fs $8.2 \pm 2.4$, En $48.7 \pm$ $5.5(\mathrm{~N}=10)$. Feldspar is Ab $81.6 \pm 5.1$, Or $5.7 \pm 1.2$, An $12.8 \pm 4.5(\mathrm{~N}=16)$. Olivine, low-Ca pyroxene, feldspar, sulfide, and metal compositions were determined with EDS point spectra. Chromite was determined through EDS phase map extracts. 
A Chemical and petrographic descriptions of individual inclusions

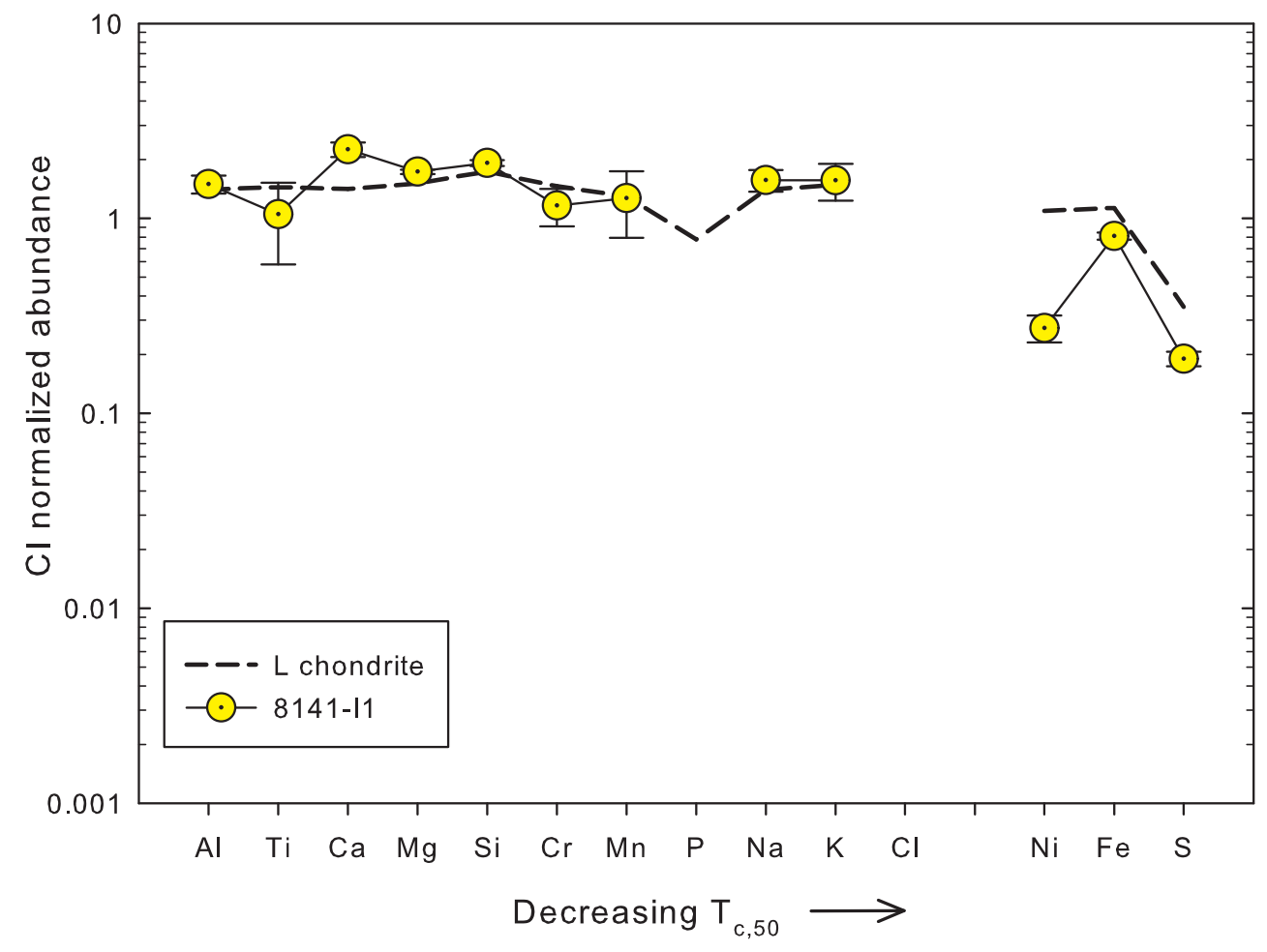

Figure A.24: CI-normalized bulk chemistry of 8141-I1. This inclusion is essentially chondritic, except for a lack of phosphate minerals and depletions of metal and sulfide.

\section{A.11 Cynthiana (AMNH 0369) L4}

One inclusion in one PTS of Cynthiana, an L4 chondrite, has been examined. 
A Chemical and petrographic descriptions of individual inclusions

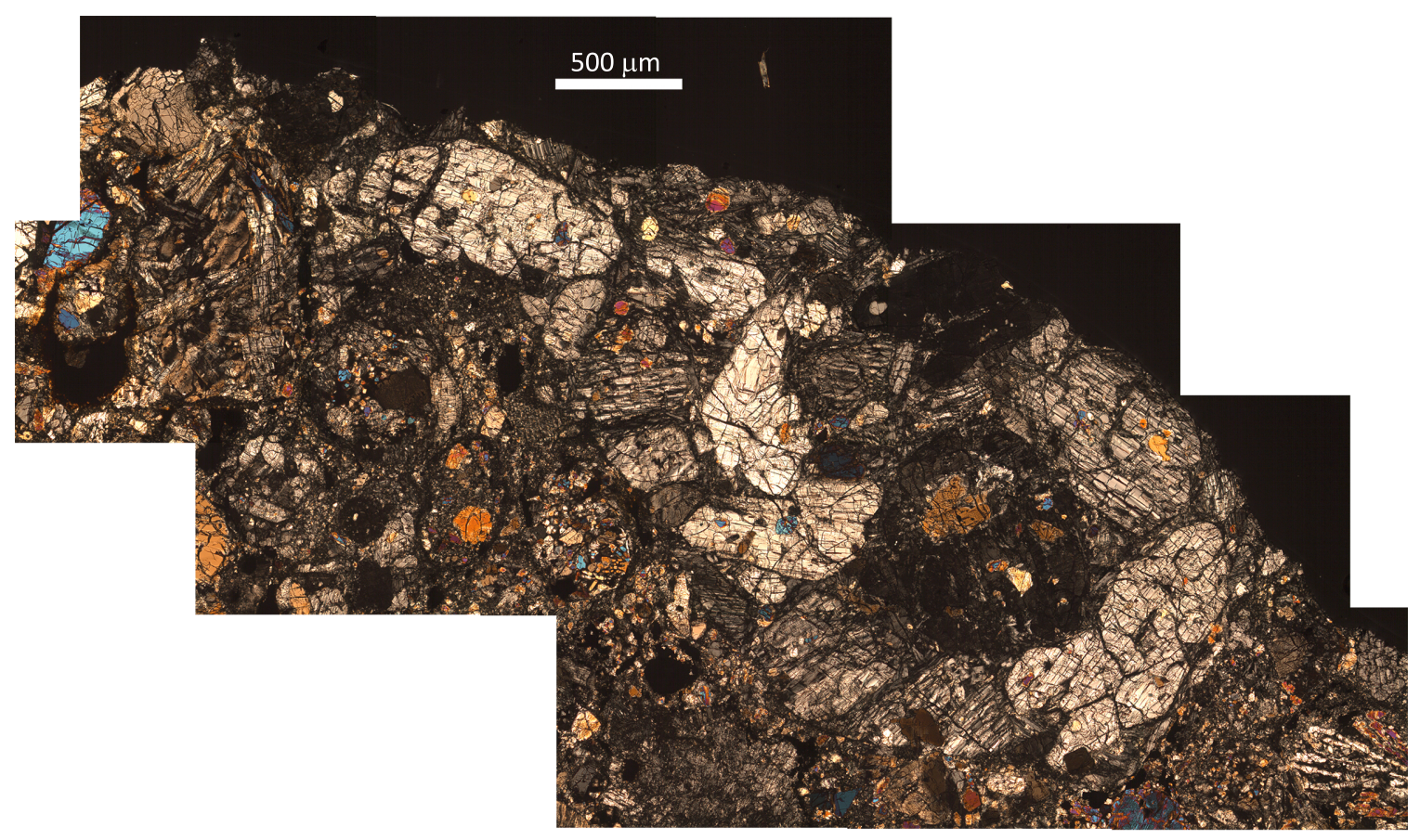

Figure A.25: Cyn-I1 under cross-polarized light

Cyn-I1 The inclusion is broken at the edge of the specimen, so its size is unclear, but its largest dimension in the thin section is $4.5 \mathrm{~mm}$. It has a sharp contact with the host, and an overall jagged, angular shape. At the contact, clinoenstatite and olivine grains are broken and jagged, suggesting the inclusion is a fragment of a larger object.

It is a pyroxene microporphyry, with subhedral low-Ca pyroxene (clinoenstatite) with some anhedral olivine phenocrysts in a groundmass of anhedral feldspar and diopside. The olivine is both individual crystals and poikilitically enclosed by the clinoenstatite. Minor chromite and taenite exists as minute $(10 \mu \mathrm{m})$ blebs throughout the inclusion, while troilite and phosphate exist as rare, coarser grains ( 50-75 $\mu \mathrm{m})$ close to the host contact. 
A Chemical and petrographic descriptions of individual inclusions

The olivine is highly equilibrated, with average composition Fa $26.9 \pm 0.3(\mathrm{~N}=$ 9). The clinoenstatite, is however, zoned, with average composition Wo $0.7 \pm 0.2$, Fs $16.9 \pm 7.7$, En $82.4 \pm 7.8(\mathrm{~N}=12)$. Diopside has average composition Wo $38.7 \pm$ 2.4, Fs $8.8 \pm 0.8$, En $52.5 \pm 2.1(\mathrm{~N}=7)$. Feldspar composition is Ab $32.8 \pm 1.9$, Or $0.4 \pm 0.1$, An $66.8 \pm 1.9(\mathrm{~N}=7)$. Olivine, low-Ca pyroxene, diopside, and feldspar compositions were determined with EDS point spectra. Sulfide, phosphate, chromite, and metal were determined from EDS phase map extracts. Ilmenite was present in quantities too small to characterize, and so was assumed pure.

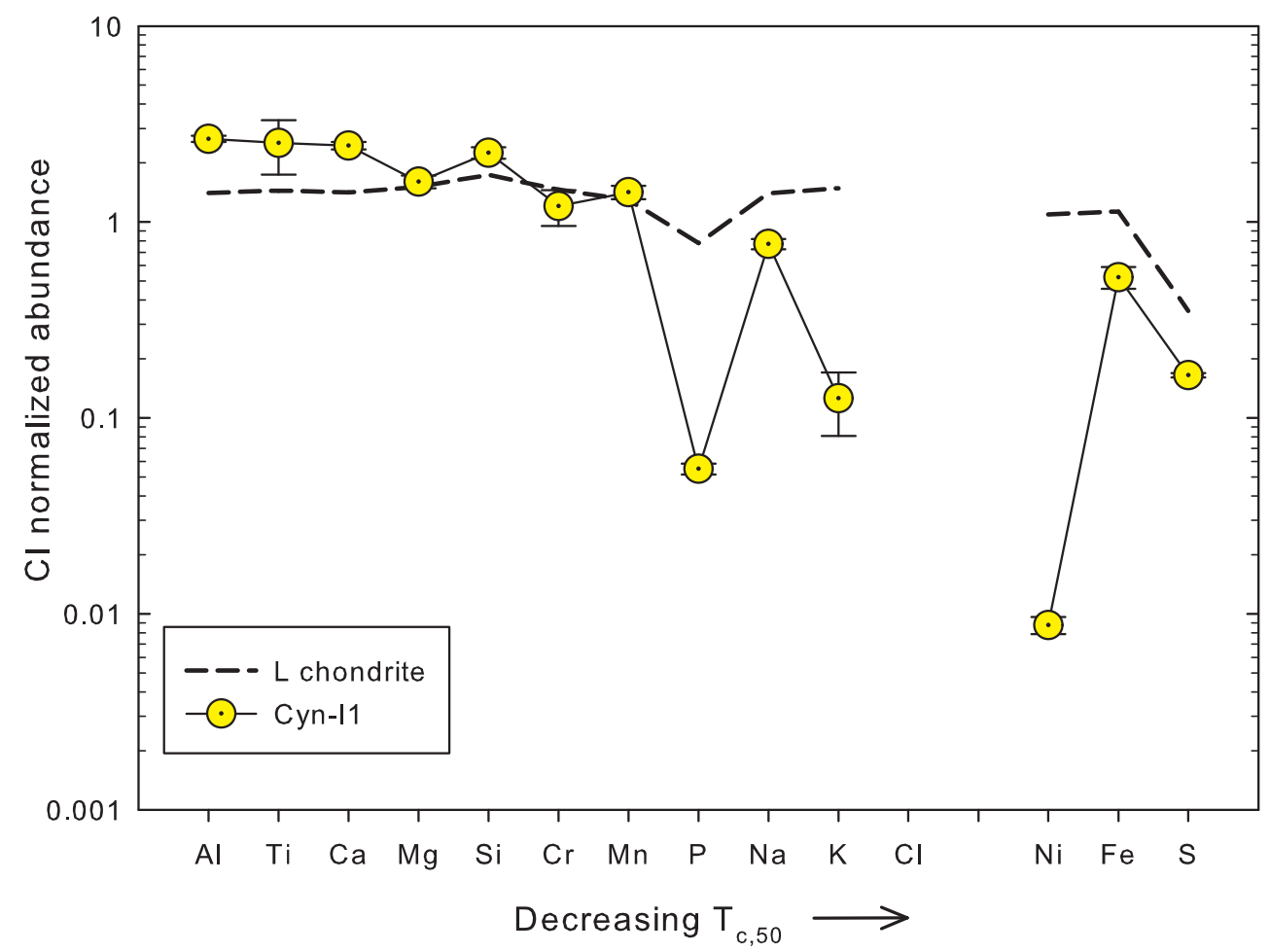

Figure A.26: CI-normalized bulk chemistry of Cyn-I1. This inclusion has an enrichment in refractory elements and a depletion in volatiles, suggesting a vapor fractionation process. 
A Chemical and petrographic descriptions of individual inclusions

\section{A.12 Kramer Creek (AMNH 4428) L4}

One inclusion from Kramer Creek, an L4 chondrite, in one PTS was examined.

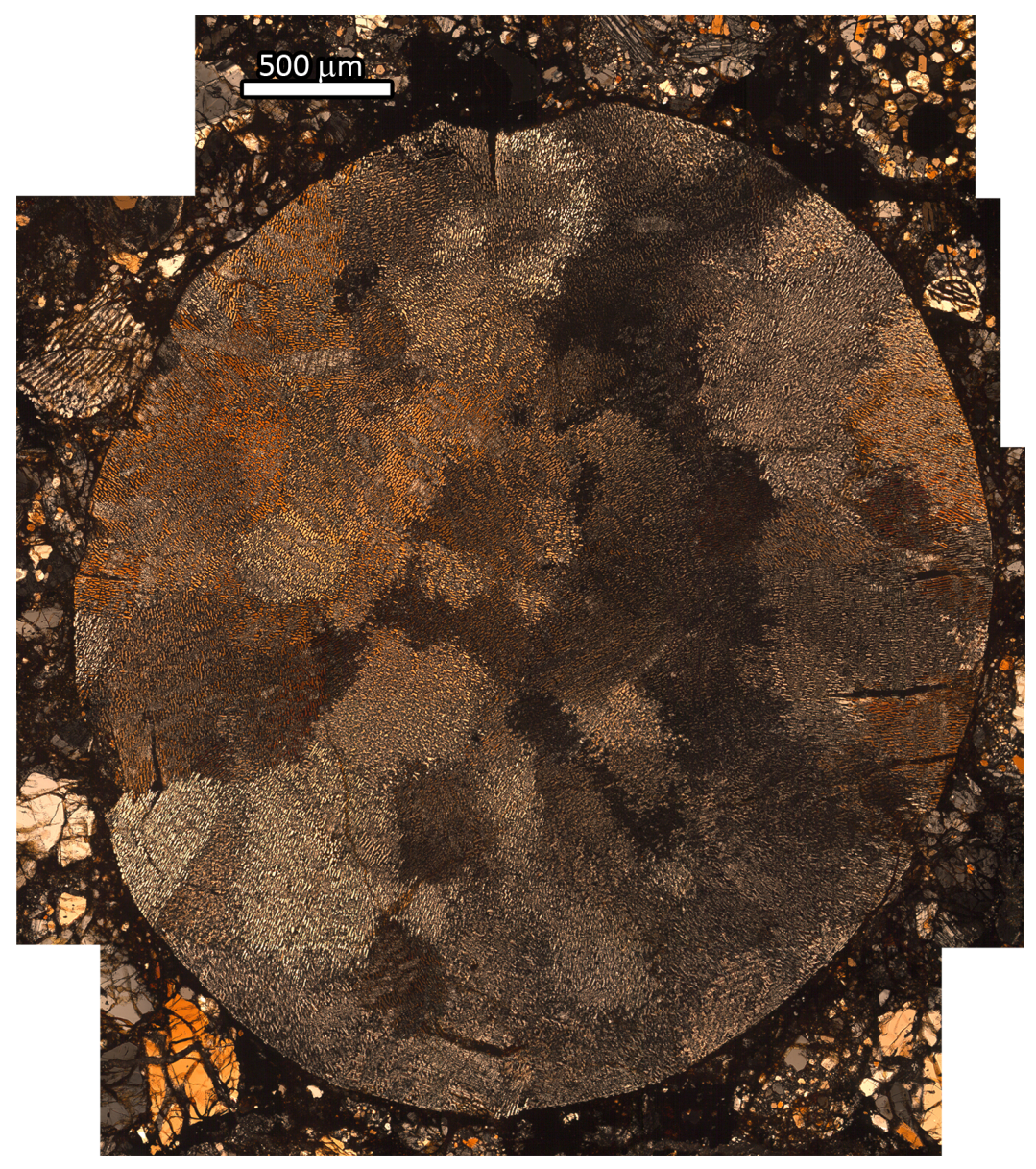

Figure A.27: Cross-polarized light micrograph of KrC-I1.

KrC-I1 This inclusion is round, about $3.5 \mathrm{~mm}$ in apparent diameter, and in sharp contact with the host. Crystals at the contact appear whole and unbroken, suggesting this edge is a primary feature. Much of the contact is delineated by weathering product from the host. Texturally, the inclusion is similar to a cryptocrystalline 
A Chemical and petrographic descriptions of individual inclusions

chondrule. Small, elongate $(\sim 10 \mathrm{x} 50 \mu \mathrm{m})$ olivine crystals that are locally mutually aligned are intergrown with pigeonite and diopside. Even finer-grained feldspar is interstitial to the olivine and pyroxene, with minor chromite/spinel, metal, sulfide, and ilmenite interstitial throughout. Smaller $(>1 \mu \mathrm{m})$ chromite/spinel blebs are suspended in the feldspar. There is no radial variation in texture or chemistry.

Olivine is highly equilibrated, with average Fa $22.0 \pm 0.5(\mathrm{~N}=20)$. The pigeonite and diopside are more variable, with average composition Wo $4.4 \pm 1.7$, Fs $18.1 \pm$ 0.5 , En $77.5 \pm 1.3(\mathrm{~N}=23)$, and Wo $45.6 \pm 2.5$, Fs $6.4 \pm 0.7$, En $48.0 \pm 1.9(\mathrm{~N}=6)$. The feldspar is even more variable, with composition Ab $64.6 \pm 8.3$, Or $1.2 \pm 0.7$, An $34.1 \pm 8.8(\mathrm{~N}=20)$. Olivine, pigeonite, diopside, feldspar, chromite, and metal were determined with EDS point spectra. Sulfide and ilmenite were present in quantities too small to analyze, and so were assumed pure. 
A Chemical and petrographic descriptions of individual inclusions

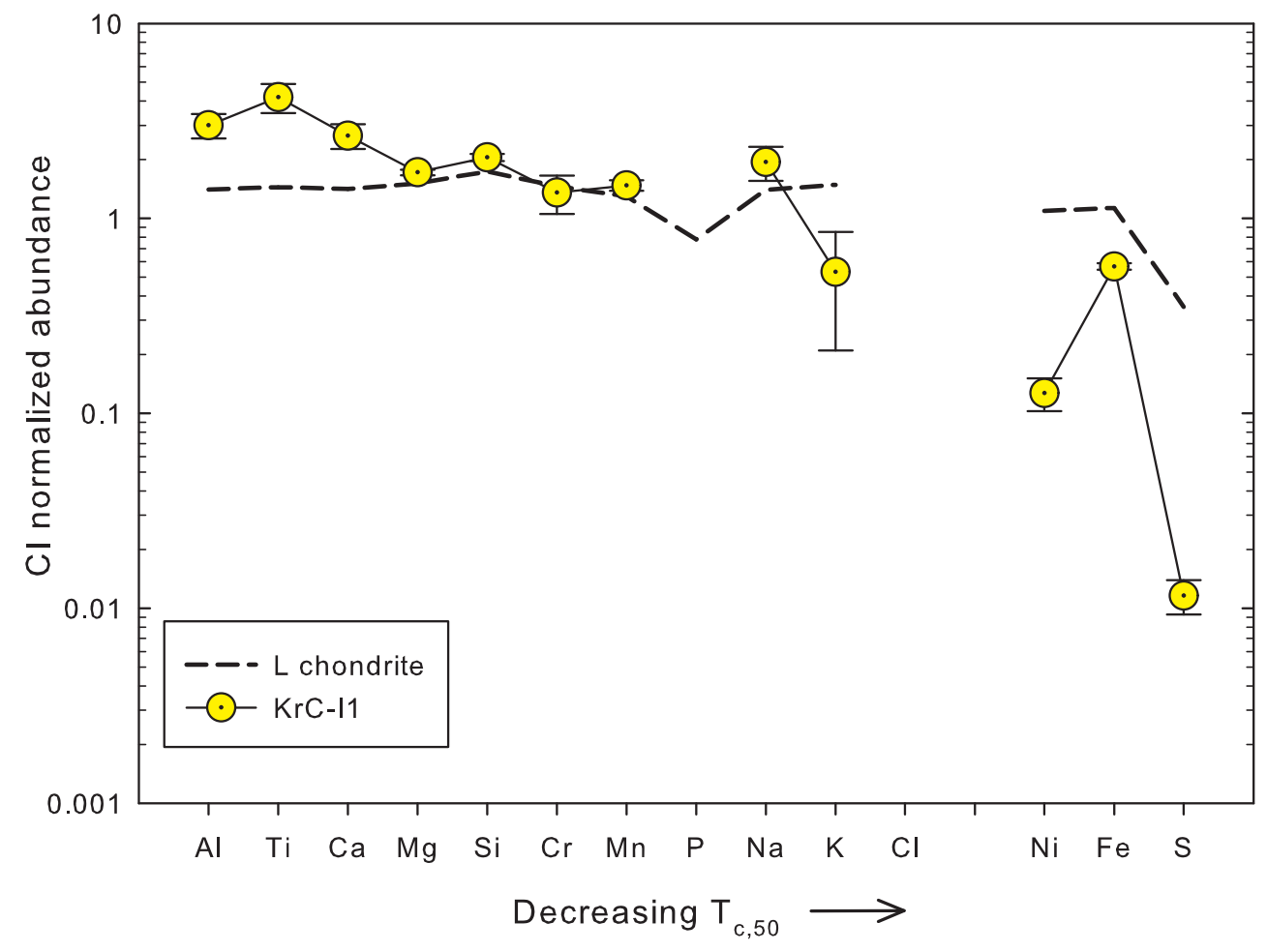

Figure A.28: CI-normalized bulk chemistry of KrC-I1. This inclusion is enriched in refractory elements and depleted in potassium, suggesting a vapor fractionation process.

\section{A.13 McKinney (AMNH 0391) L4}

McKinney is an L4 chondrite. One PTS containing two inclusions has been examined. 
A Chemical and petrographic descriptions of individual inclusions

\section{A.13.1 McK-I1}

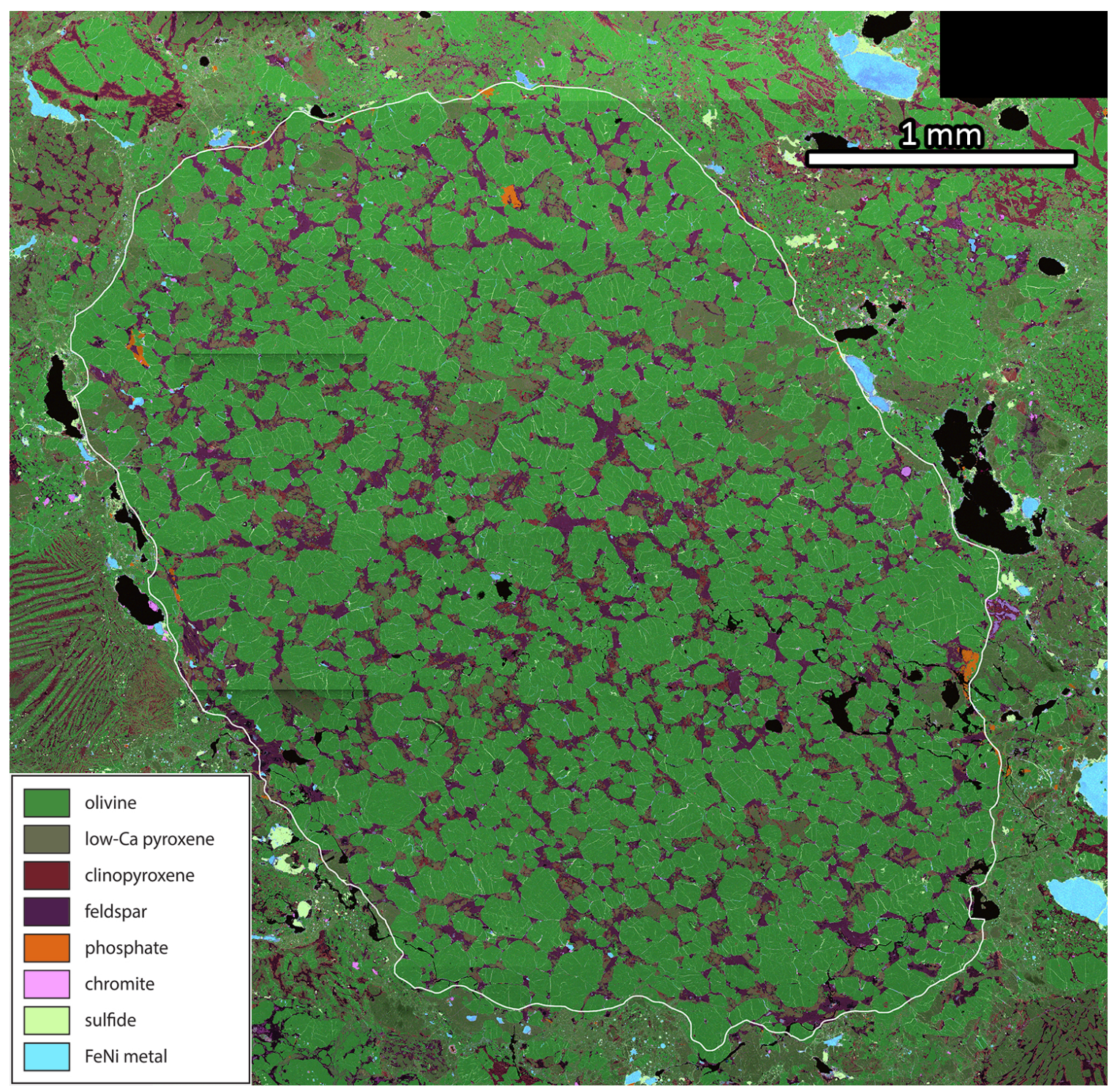

Figure A.29: False-color EDS map of McK-I1.

This inclusion is oval shaped, $4 \mathrm{~mm} \times 2.75 \mathrm{~mm}$ in apparent size. It is in sharp contact with the host, and many crystals at the contact are broken, suggesting this 
A Chemical and petrographic descriptions of individual inclusions

is a fragment of a larger object. Much of the contact is delineated by an opaque vein of shock melted material. It is an olivine microporphyry, the subhedral olivine phenocrysts that range in size from $\sim 50 \mu \mathrm{m}$ to $\sim 300 \mu \mathrm{m}$. The phenocrysts are likely relicts that did not melt with the rest of the inclusion material, as they are extensively fractured. and the fractures have filled with metal and sulfide.

Interstitial to the olivine is pigeonite, diopside, and both anorthitic feldspar and a glassy feldspathic or maskelynite phase. Small chromite, phosphate, sulfide, and FeNi metal grains are present throughout the inclusion.

There are at least two curved crystals of olivine, that may be fragments of olivine "donuts," as well as several olivine "shells" (see section 4.4), which are large olivine crystals that enclose an assemblage of other silicates.

Olivine is equilibrated, with average composition Fa $25.8 \pm 0.2(\mathrm{~N}=20)$. The pigeonite and diopside are more variable in composition, with compositions Wo 13.1 \pm 3.3 , Fs $20.9 \pm 2.3$, En $66.0 \pm 3.0(\mathrm{~N}=18)$ and Wo $27.7 \pm 4.3$, Fs $15.1 \pm 1.9$, En $57.3 \pm 2.9(\mathrm{~N}=6)$, respectively. The feldspar and glass have compositions $\mathrm{Ab}$ $21.8 \pm 2.8$, Or $0.4 \pm 0.2$, An $77.7 \pm 2.9(\mathrm{~N}=16)$ and $\mathrm{Ab} 87.5 \pm 1.8$, Or $4.8 \pm 0.8$ An $7.7 \pm 2.0(\mathrm{~N}=4)$, respectively. Olivine, pigeonite, diopside, both feldspars, and chromite compositions were determined with EDS point spectra. Phosphate, metal, and sulfide compositions were determined with AZtec's analyze phases. 
A Chemical and petrographic descriptions of individual inclusions

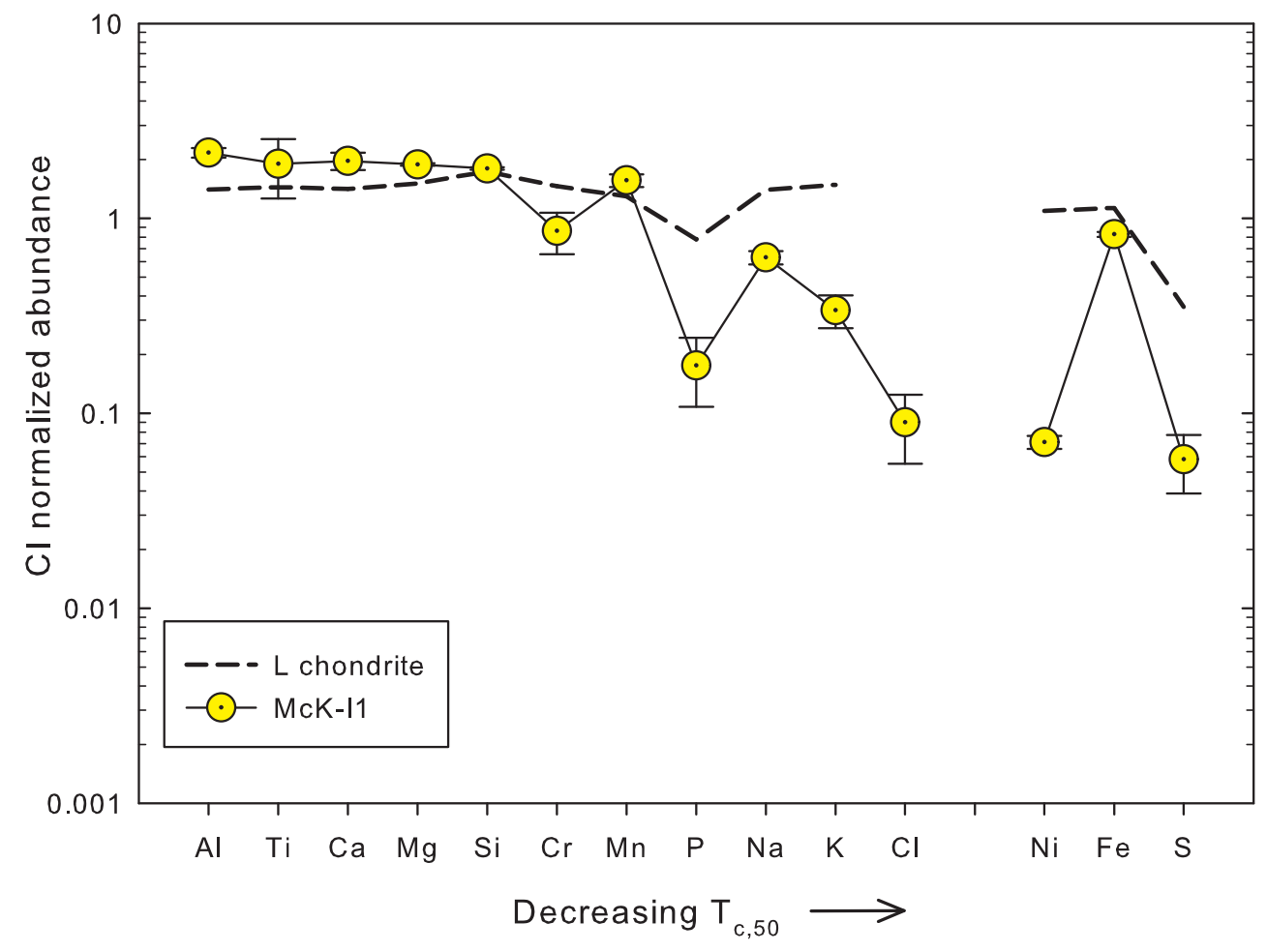

Figure A.30: CI-normalized bulk chemistry of McK-I1. This inclusion is depleted in the more volatile elements, suggesting a vapor-fractionation process. 
A Chemical and petrographic descriptions of individual inclusions

\section{A.13.2 McK-I2}

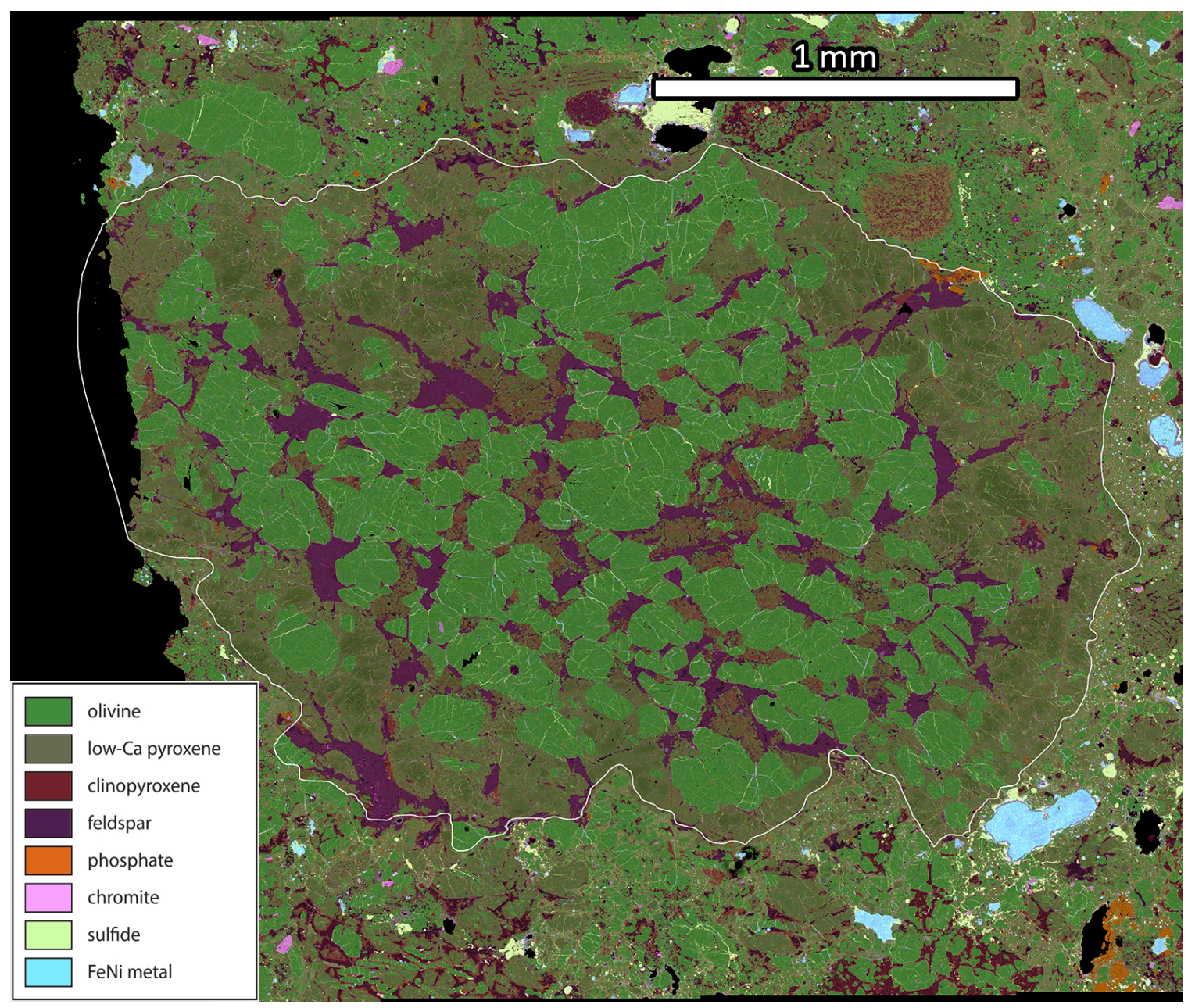

Figure A.31: False-color EDS phase map of McK-I2.

The second inclusion is texturally very similar to the first. It is rounded and broken at the edge of the sample, but its apparent diameter is $\sim 3 \mathrm{~mm}$. It is in sharp contact with the host, and much of the contact is delineated by shock-melted material. Some of the inclusion material appears broken at the contact, but there is a nearly unbroken rim of low-Ca pyroxene, suggesting that this edge is a primary feature of the inclusion. 
A Chemical and petrographic descriptions of individual inclusions

There is a subtle radial variation in mineralogy. There is coarse $(\sim 50-400 \mu \mathrm{m})$, anhedral to subhedral olivine throughout the inclusion, which is intergrown with pyroxene. In the center of the inclusion, the pyroxene phase is anhedral pigeonite, on average $\sim 50 \mu \mathrm{m}$ in size. As mentioned above the outer region of the inclusion is dominated by low-Ca pyroxene, which is coarser $(\sim 300 \mu \mathrm{m})$, and zoned. Both anorthite and a feldspathic glass are interstitial to the olivine and pyroxene throughout the inclusion. The olivine and low-Ca pyroxene are fractured, and the fractures have filled with sulfide and some minor metal. Conspicuously, neither the pigeonite or feldspar are fractured. Minute $(\sim 10 \mu \mathrm{m})$ chromite grains are throughout, primarily enclosed by the feldspar. Minor amounts of phosphate are interstitial to the low-Ca pyroxene near the host contact.

The chemistry of this inclusion, determined through extracting data from an EDS map, is different from the other inclusion. The olivine is similar, with average Fa $24.5 \pm 0.6(\mathrm{~N}=7)$, but the two pyroxene phases have compositions Wo $2.6 \pm 0.6$, Fs $18.1 \pm 2.2$, En $79.3 \pm 2.7(\mathrm{~N}=6)$ and Wo $18.9 \pm 3.0$, Fs $16.6 \pm 1.1$, En 64.5 $\pm 2.4(\mathrm{~N}=4)$, which are less calcic than the pyroxene of the first inclusion. Like McK-I2, however, both an anorthitic feldspar (Ab $14.7 \pm 1.0$, Or $0.5 \pm 0.4$, An 84.8 $\pm 1.2, \mathrm{~N}=6$ ) and a feldspathic glass (sodic, but not stoichimetrically feldspar) exist. Compositions of olivine, low-Ca pyroxene, pigeonite, feldspar, and the glass were determined with EDS phase map extracts. Phosphate chemistry was determined with AZtec's analyze phases. Chromite, metal, and sulfide were present in quantities too small to characterize, and so were assumed pure. 
A Chemical and petrographic descriptions of individual inclusions

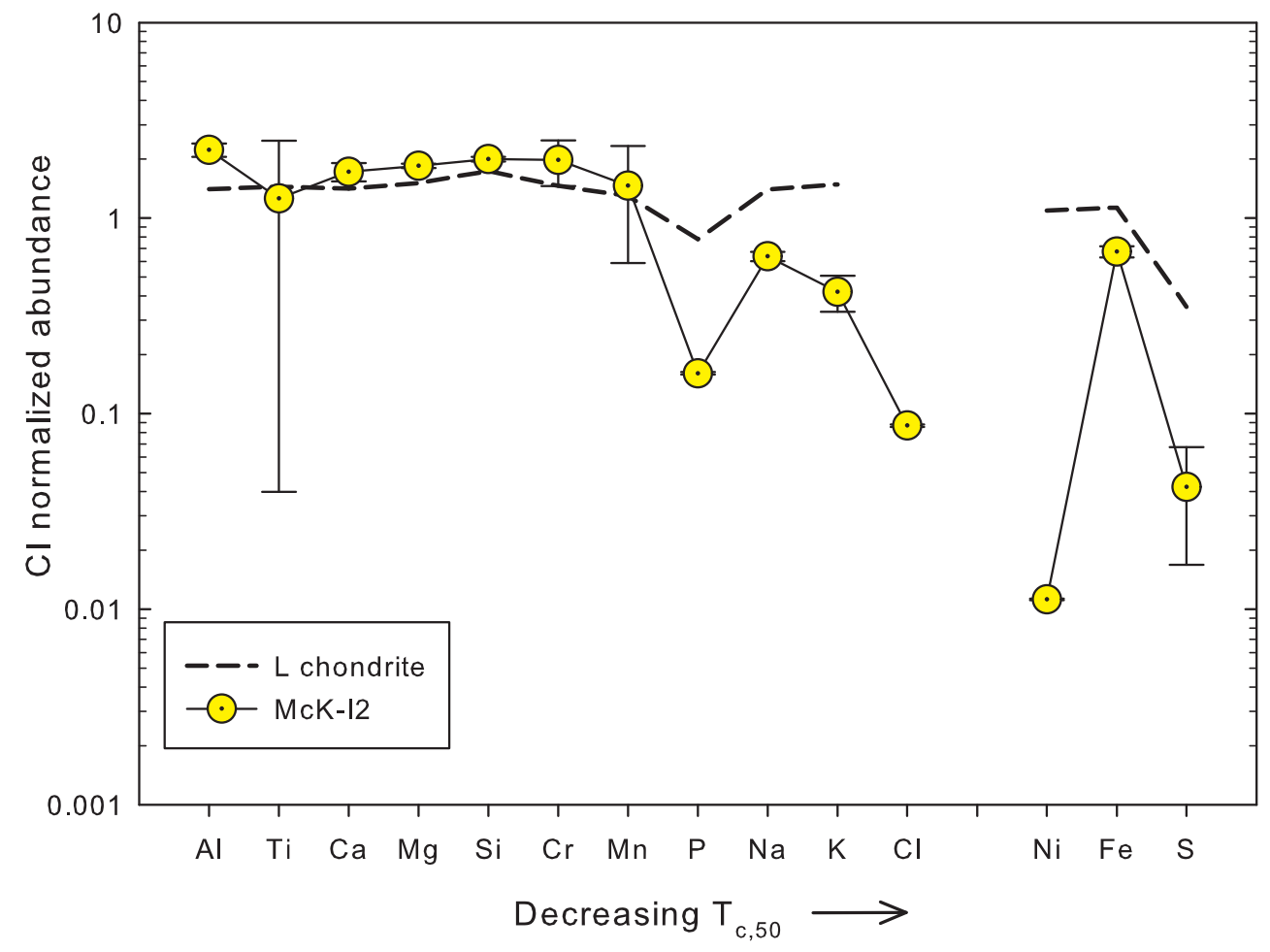

Figure A.32: CI-normalized bulk chemistry of McK-I2. This inclusion is depleted in volatile elements, suggesting a vapor fractionation process.

\section{A.14 NWA 7870 (CML 0173) L4}

NWA 7870 is an L4 chondrite. One polished thin section of NWA 7870 containing one inclusion was examined. 
A Chemical and petrographic descriptions of individual inclusions

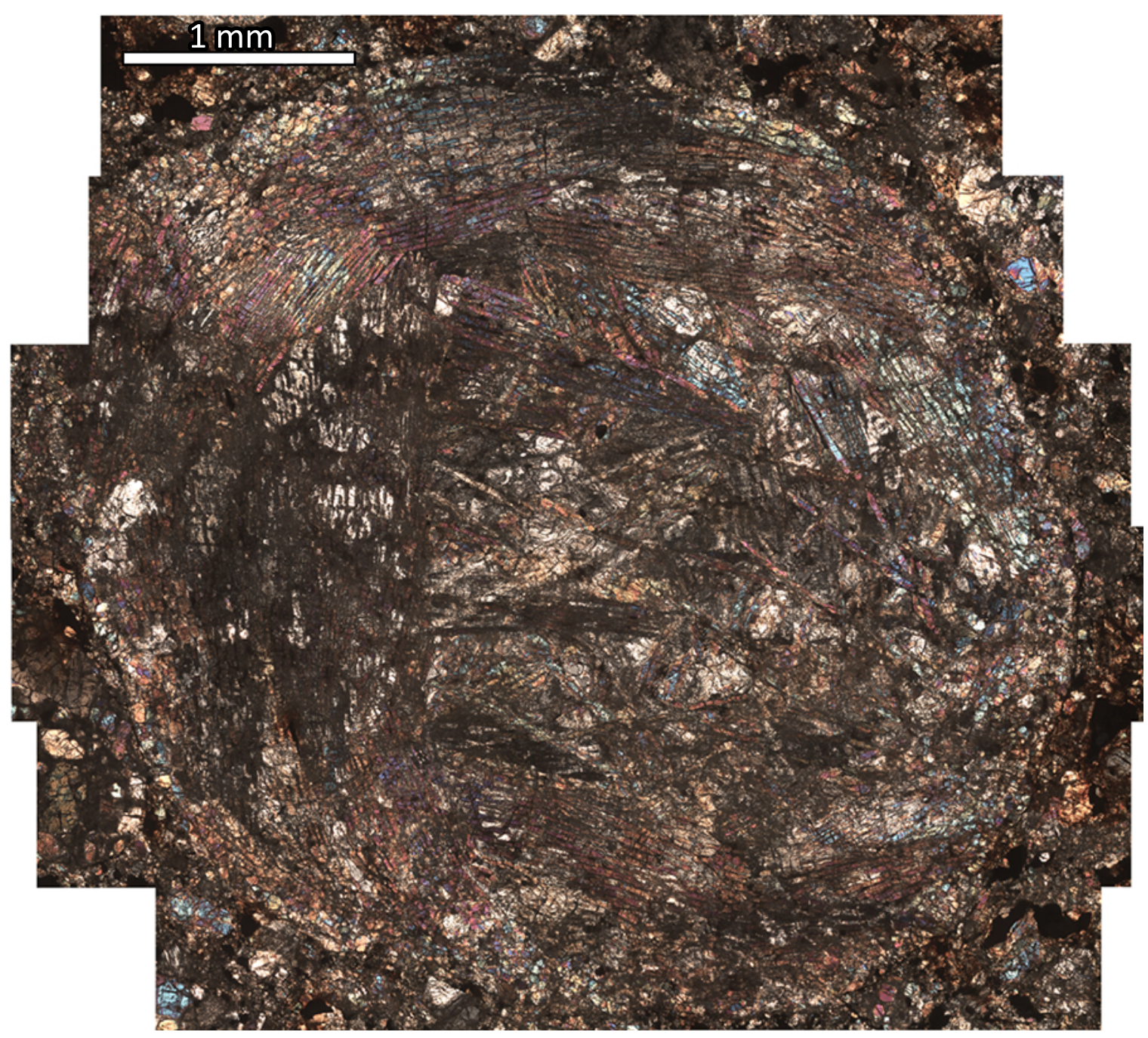

Figure A.33: NWA 7870-1 under cross-polarized light.

NWA 7870-I1 The inclusion is round, with an apparent diameter of approximately $5 \mathrm{~mm}$, and has a sharp, curved contact with the host. Grains at the contact do not appear broken, suggesting that this inclusion is not a fragment of a larger object. Its texture is dominated by skeletal olivine, though it varies radially, with smaller, rounded anhedral olivine crystals concentrated at the edge (see figure A.33).

The mineralogy is primarily olivine, which occurs as skeletal crystals on average 
A Chemical and petrographic descriptions of individual inclusions

$500 \mu \mathrm{m}$ long by $20 \mu \mathrm{m}$ wide, and as rounded, anhedral grains that average $50 \mu \mathrm{m}$ in diameter, and low-Ca pyroxene, which is interstitial to the skeletal olivine. Anhedral albitic plagioclase and diopside are also interstitial to the olivine. Chlorapatite is present as relatively coarse $(\sim 100 \mu \mathrm{m})$ grains, and metal occurs as both kamacite and taenite in small $(5-50 \mu \mathrm{m})$ blebs. Minute blebs of chromite $(\sim 5 \mu \mathrm{m})$ are throughout the inclusion, and one tiny ilmenite grain $(\sim 10 \mu \mathrm{m})$ was found. Sulfide is entirely absent, or only present in quantities that are below the detection limit, though it should be noted that only one thin section was analyzed, and as such it is possible that a sulfide phase is present elsewhere in the inclusion.

The inclusion is largely equilibrated; phase composition does not vary within the inclusion, nor is there any zoning within any of the crystals. Olivine composition is on average Fa $25.1 \pm 0.4(\mathrm{~N}=29)$, which is the same range as the L-chondrite host meteorite. Low-Ca pyroxene has average composition En $76.6 \pm 0.8$, Fs 21.5 \pm 0.6 , Wo $1.9 \pm 0.6(\mathrm{~N}=38)$, which is more equilibrated and higher in $\mathrm{FeO}$ than the host. The diopside has average composition En $47.7 \pm 1.4$, Fs $8.1 \pm 0.6$, Wo $44.3 \pm 2.0(\mathrm{~N}=13)$. Olivine composition was determined with EMPA and EDS point spectra. Low-Ca pyroxene, diopside, and feldspar were characterized with EDS point chemistry. Phosphate and metal were determined with EDS map extracts and AZtec's analyze phases. Chromite was present in quantities too small to analyze, and so was assumed pure. 
A Chemical and petrographic descriptions of individual inclusions

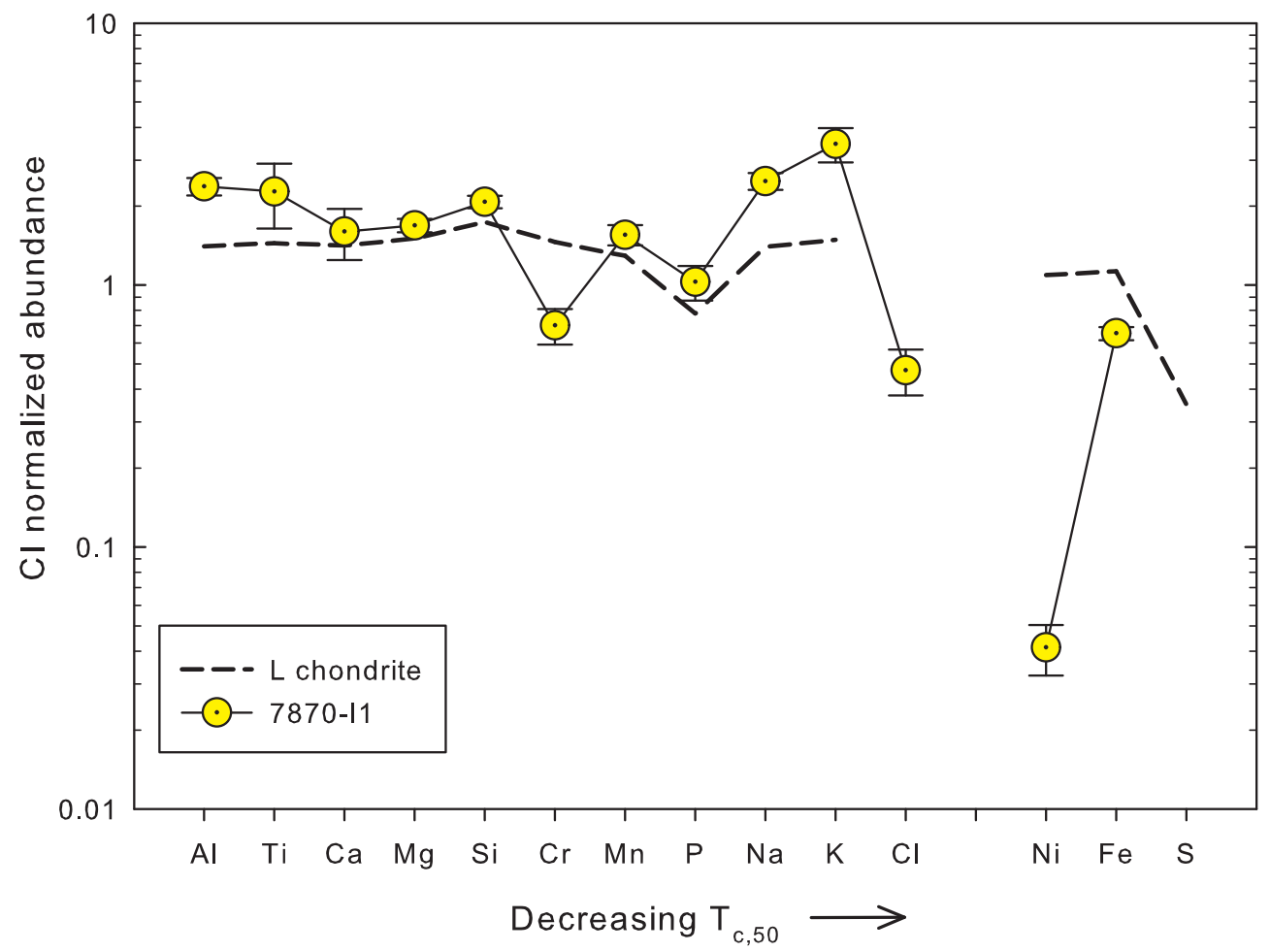

Figure A.34: CI-normalized bulk chemistry of 7870-I1. This inclusion is enriched in the alkali elements, as well as aluminum, suggesting an enrichment in feldspar (see section 4.3.2).

\section{A.15 NWA 7871 (CML 0178) L6}

One PTS of NWA 7871, which is an L6 chondrite, containing one exceptionally large inclusion has been examined. 
A Chemical and petrographic descriptions of individual inclusions

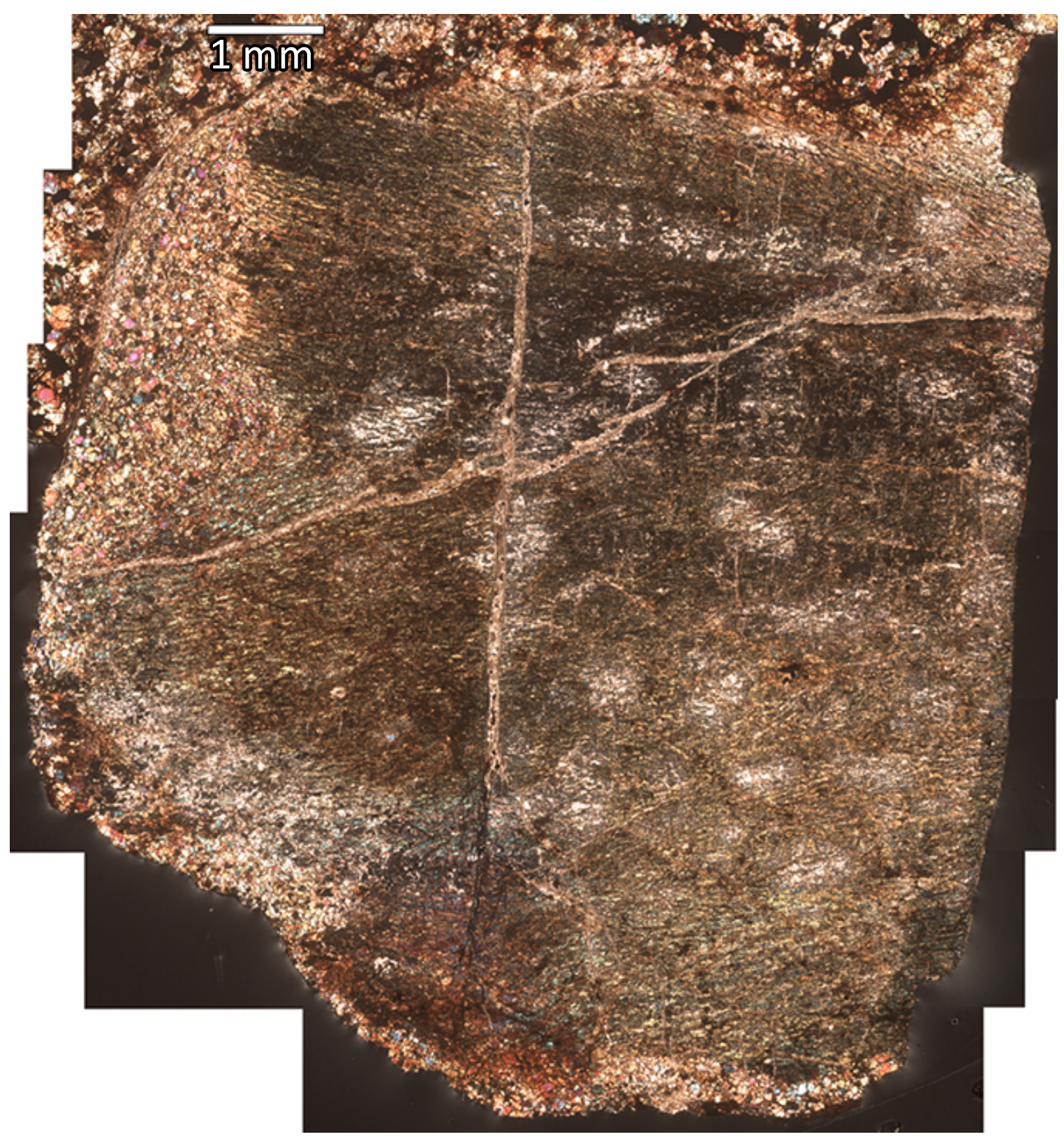

Figure A.35: 7871-I1 under cross-polarized light.

NWA 7871-I1 The inclusion is subrounded and squarish, and quite large, with a diameter of approximately $9.5 \mathrm{~mm}$. Its contact with the host is sharp, and crystals at the contact appear whole and unbroken. By volume, the inclusion is over $70 \%$ olivine, 
A Chemical and petrographic descriptions of individual inclusions

which is uniformly fine-grained. Grains are on average $35 \mu \mathrm{m}$ in diameter, with a range of 20-40 $\mu \mathrm{m}$, and most crystals are slightly elongated and share an orientation and extinction with other local grains. There is a change in texture towards the edge; olivine crystals are larger, granular, and have a greater size distribution towards the contact with the host. The inclusion is crossed by several fractures or veins that do not extend into the host, some of which offset each other. These veins contain calcite and phosphate, which also occur in the host.

Feldspar and minute blebs $(\sim 10 \mu \mathrm{m})$ of chromite, troilite, and FeNi metal is interstitial to the olivine throughout the inclusion. Diopside is interstitial to the olivine in patches, as is a large area of both chlorapatite and merrillite (see figure A.35).

The olivine is highly equilibrated, with average $\mathrm{Fa}$ of $25.6 \pm 0.3(\mathrm{~N}=15)$, consistent with an $\mathrm{L}$ chondrite. As the host meteorite is a high petrologic type, this equilibration could have occurred either before or after incorporation into the host. Diopside is also equilibrated but less so, with composition Wo $46.1 \pm 1.3$, Fs $8.0 \pm$ 0.5 , En $45.9 \pm 1.7(\mathrm{~N}=7)$. Feldspar is somewhat variable in composition, with $\mathrm{Ab}$ $78.8 \pm 3.9$, Or $6.1 \pm 0.9$, An $15.1 \pm 3.3(\mathrm{~N}=5)$. Olivine was characterized with EMPA and EDS point spectra. Diopside and feldspar were characterized with EDS point spectra, EDS map extracts, and AZtec's analyze phases. Metal and phosphate compositions were determined with AZtec's analyze phases. 
A Chemical and petrographic descriptions of individual inclusions

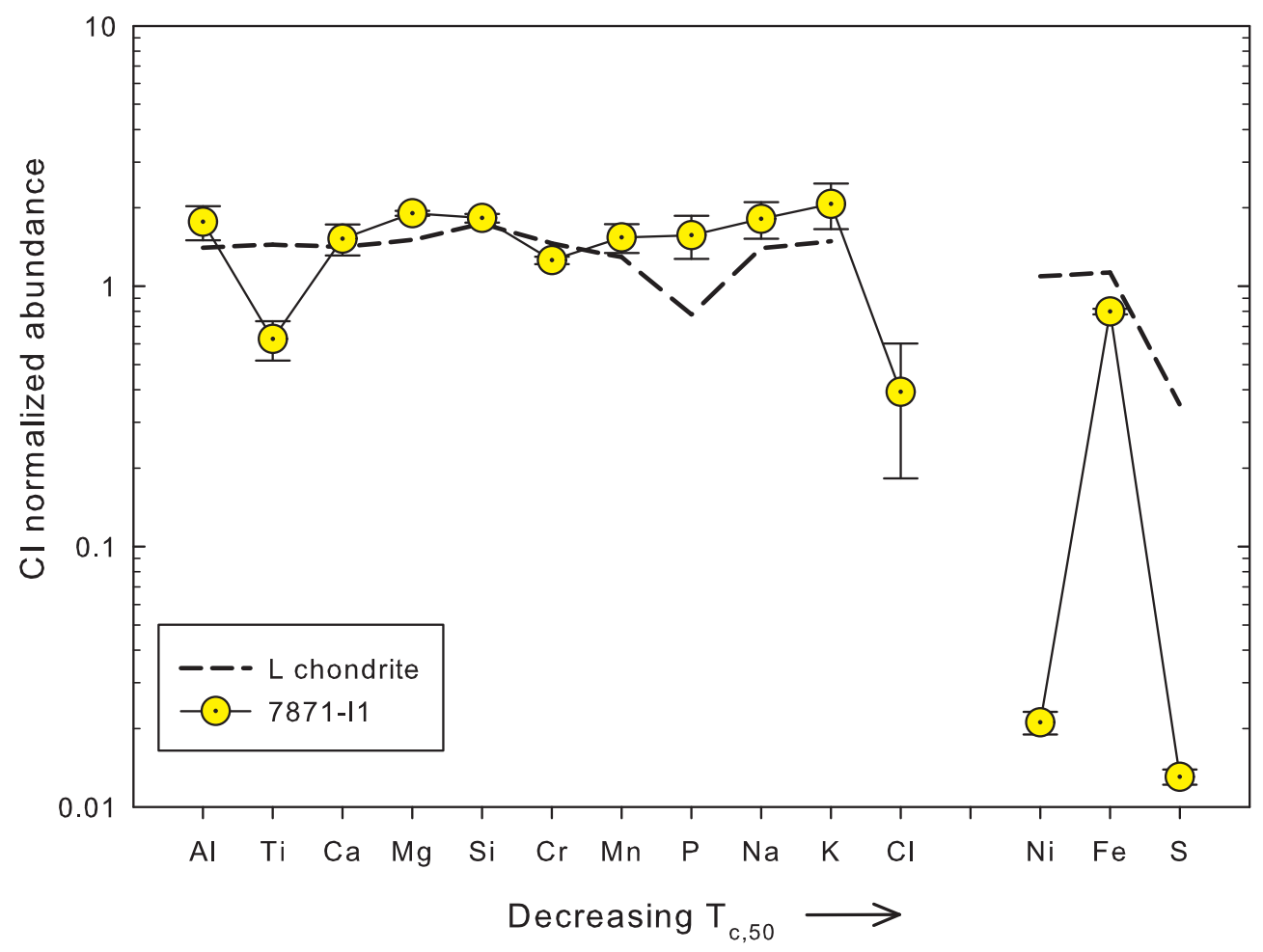

Figure A.36: CI-normalized bulk chemistry of 7871-I1. This inclusion is essentially chondritic.

\section{A.16 Palo Verde Mine (CML 0185) L6}

Palo Verde Mine is an L6 chondrite. One polished thin section containing one inclusion has been examined. 
A Chemical and petrographic descriptions of individual inclusions

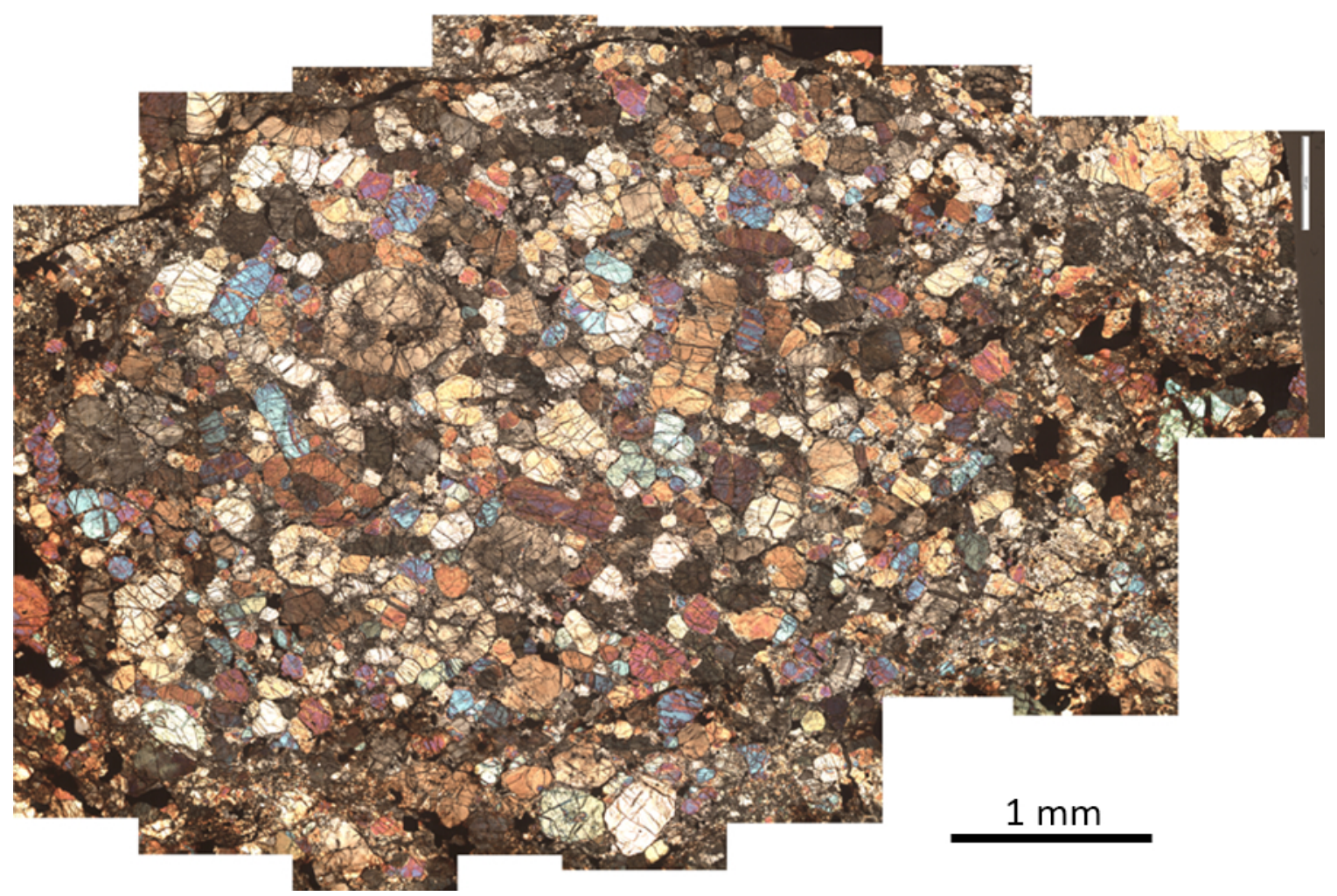

Figure A.37: PVM-I1 under cross-polarized light.

PVM-I1 The inclusion is oval-shaped, approximately $4.5 \times 6.5 \mathrm{~mm}$. One one side, the contact with the host is defined by a jagged shock vein, though the rest of the host contact is less clear. Crystals at the contact do not appear broken.

It has a porphyritic texture, with subhedral to anhedral olivine phenocrysts that have a mean size of $200 \mu \mathrm{m}$ and a range of $50-700 \mu \mathrm{m}$. Interstitial to the olivine is sodic maskelynite that embays anhedral diopside and olivine crystals. The diopside grains are smaller $(\sim 10 \mu \mathrm{m})$ in the center of the inclusion, and get larger towards the edges $(\sim 100 \mu \mathrm{m})$. A band of maskelynite also surrounds the inclusion, and in some areas the maskelynite contains clusters of very minute $(<10 \mu \mathrm{m})$ blebs of chromite. A small amount of anhedral low-Ca pyroxene is present at the host contact. 
A Chemical and petrographic descriptions of individual inclusions

Chlorapatite phosphate, metal, and zoned chromite-spinel are present throughout the inclusion but are concentrated in the outer $\sim 1.5 \mu \mathrm{m}$. In the interior, these phases only exist as minute $(<10 \mu \mathrm{m})$ blebs, but nearer the edges occur as large as $>100$ $\mu \mathrm{m}$.

The olivine is highly equilibrated, with $\mathrm{Fa}=25.5 \pm 0.4(\mathrm{~N}=25)$; low-Ca pyroxene equilibrated but less so, with Wo $1.9 \pm 1.0$, Fs $20.9 \pm 1.3$, En $77.2 \pm 0.8$ $(\mathrm{N}=6)$, both of which are consistent with an $\mathrm{L}$ chondrite. As the host itself is a high petrologic type, this equilibration could have occurred either before or after the inclusion was incorporated into the host. The diopside is also equilibrated, with Wo $45.1 \pm 0.9$, Fs $7.3 \pm 0.3$, En $47.3 \pm 0.6(\mathrm{~N}=5)$. The maskelynite varies mostly in its $\mathrm{K}$ content, with $\mathrm{Ab} 82.9 \pm 1.9$, Or $5.9 \pm 2.1$, An $11.2 \pm 0.5(\mathrm{~N}=10)$. Olivine composition was determined by EMPA and EDS point spectra. Diopside and feldspar were characterized with EDS point spectra. Low-Ca pyroxene, phosphate, and metal were determined from EDS map extracts.

This inclusion is one of many that contain olivine "donuts" (see section 4.4). There are at least 10 donuts visible in this PTS, with average diameter of $\sim 500 \mu \mathrm{m}$. None exhibit the obvious barred olivine texture common in other donuts, but have at most one "bar" of olivine across the interior of the shell. Otherwise they are hollow shells of olivine that contain small diopside and olivine crystals in a feldspathic groundmass.

This inclusion is also one of several that has a nearby anomalously large metal and sulfide grain. 
A Chemical and petrographic descriptions of individual inclusions

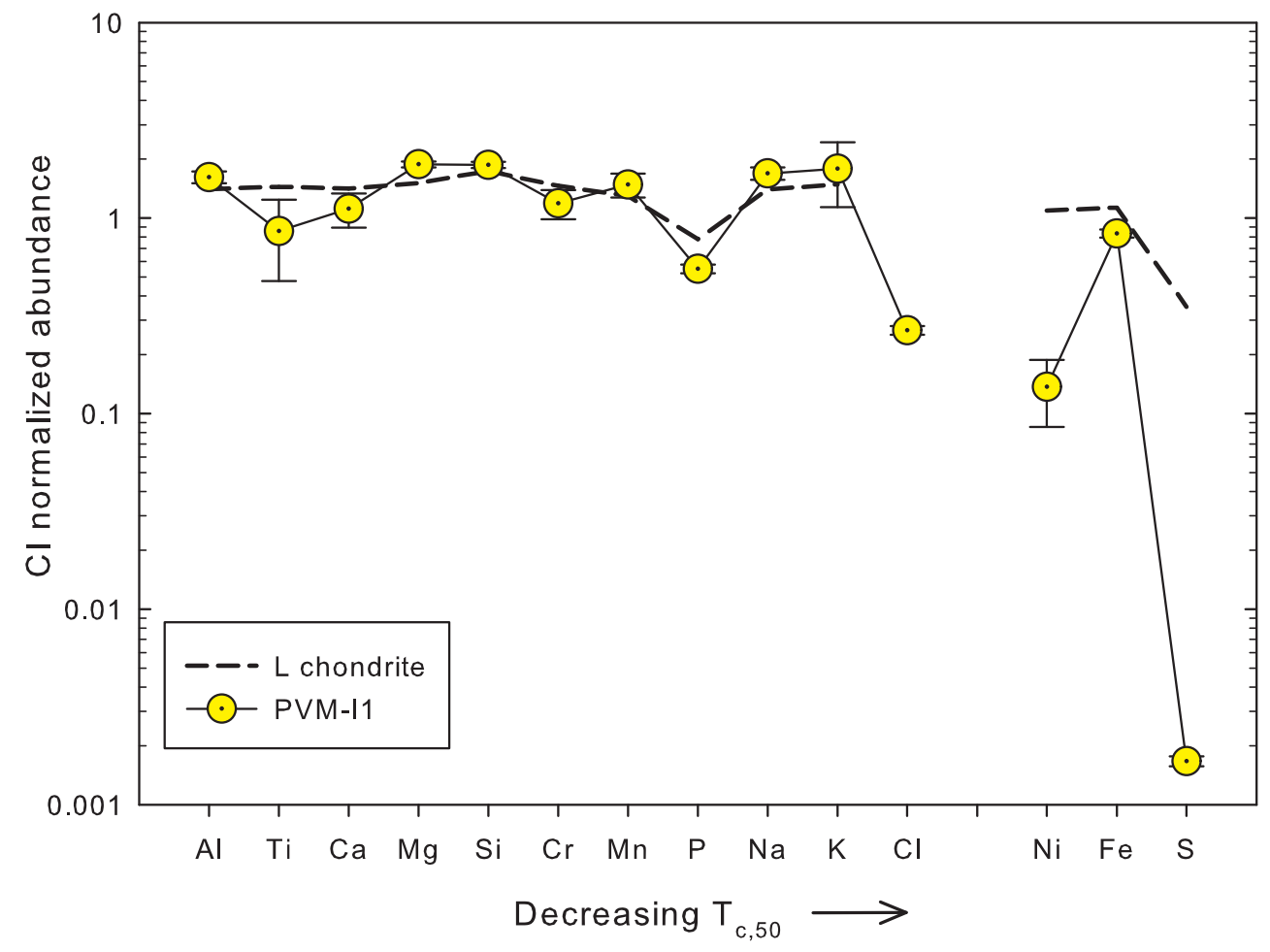

Figure A.38: CI-normalized bulk chemistry of PVM-I1. This inclusion is essentially chondritic, except for a strong depletion of metal and sulfide.

\section{A.17 Ragland (AMNH 4825) LL3.4}

One inclusion from Ragland, an LL3.4 chondrite, in one thin section has been examined. 


\section{Rgl-I1}

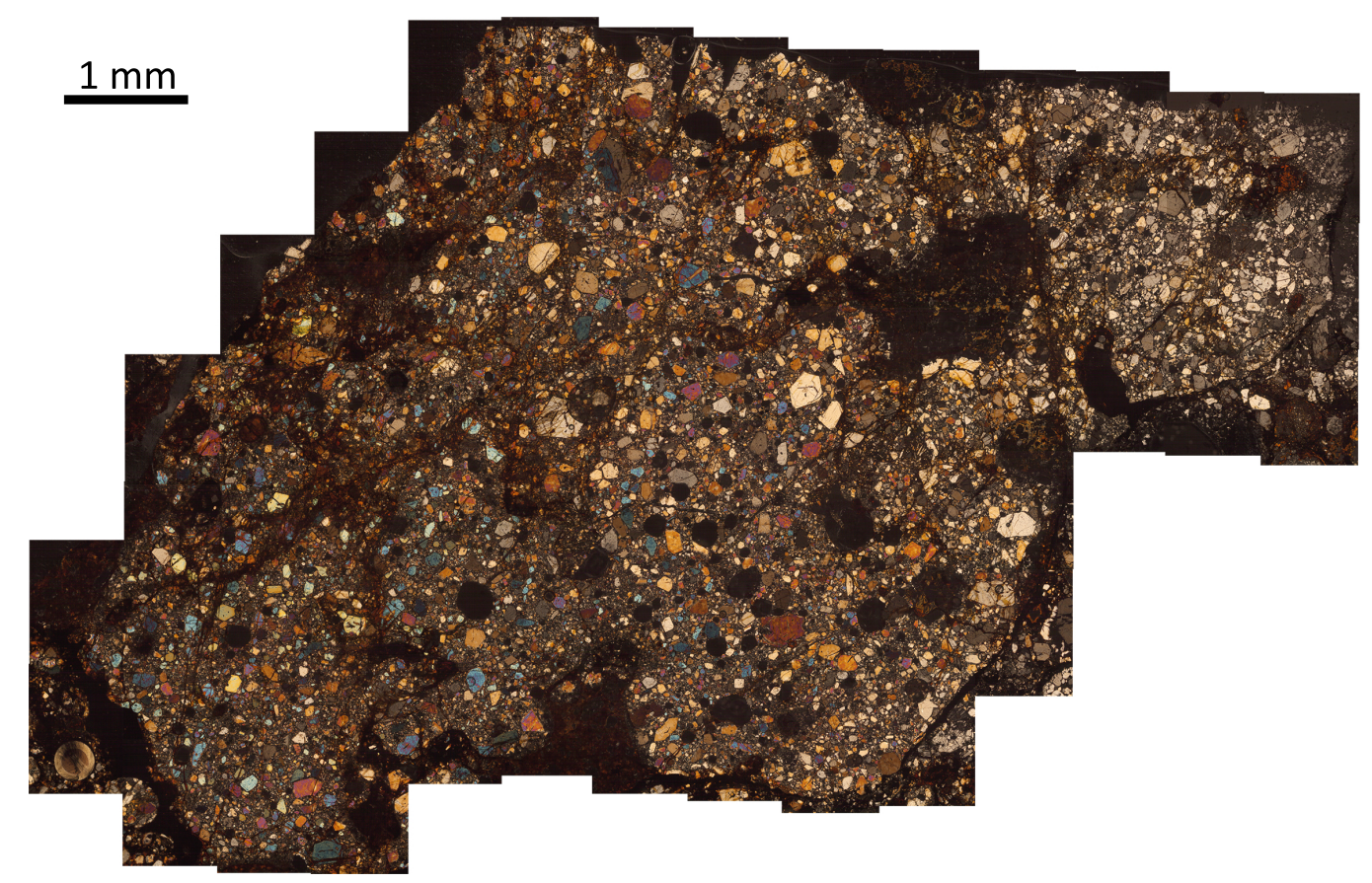

Figure A.39: Cross-polarized optical light micrograph of Rgl-I1.

This inclusion is quite large, but heavily weathered. It is broken at the edge of the specimen, so its original size is unknown, but its largest observable dimension is 1.2 $\mathrm{cm}$, and based on its curvature was probably much larger. It is in sharp contact with the host, with much of the contact (that isn't obscured by weathering) delineated by a shock vein.

It is an olivine microporphyry, with euhedral olivine phenocrysts that have a large size distribution (ranging from 10 to $500 \mu \mathrm{m}$ ). The olivine is set in a mesostasis of feldspathic glass and microlites that are below the detection limit of the dataset. Minute $(\sim 10 \mu \mathrm{m})$ chromite, sulfide, and FeNi metal grains are throughout the inclusion. 
A Chemical and petrographic descriptions of individual inclusions

The olivine phenocrysts larger than $\sim 50 \mu \mathrm{m}$ are zoned, with magnesian cores and ferroan rims, while smaller olivines are the same composition as the rims of the larger. Average olivine chemistry is Fa $22.0 \pm 7.2(\mathrm{~N}=21)$. Olivine and mesostasis compositions were determined from EDS phase map extracts, while chromite, sulfide, and metal were characterized with AZtec's analyze phases.

The inclusion is brecciated, crossed by many fracture zones that do not appear to extend into the host.

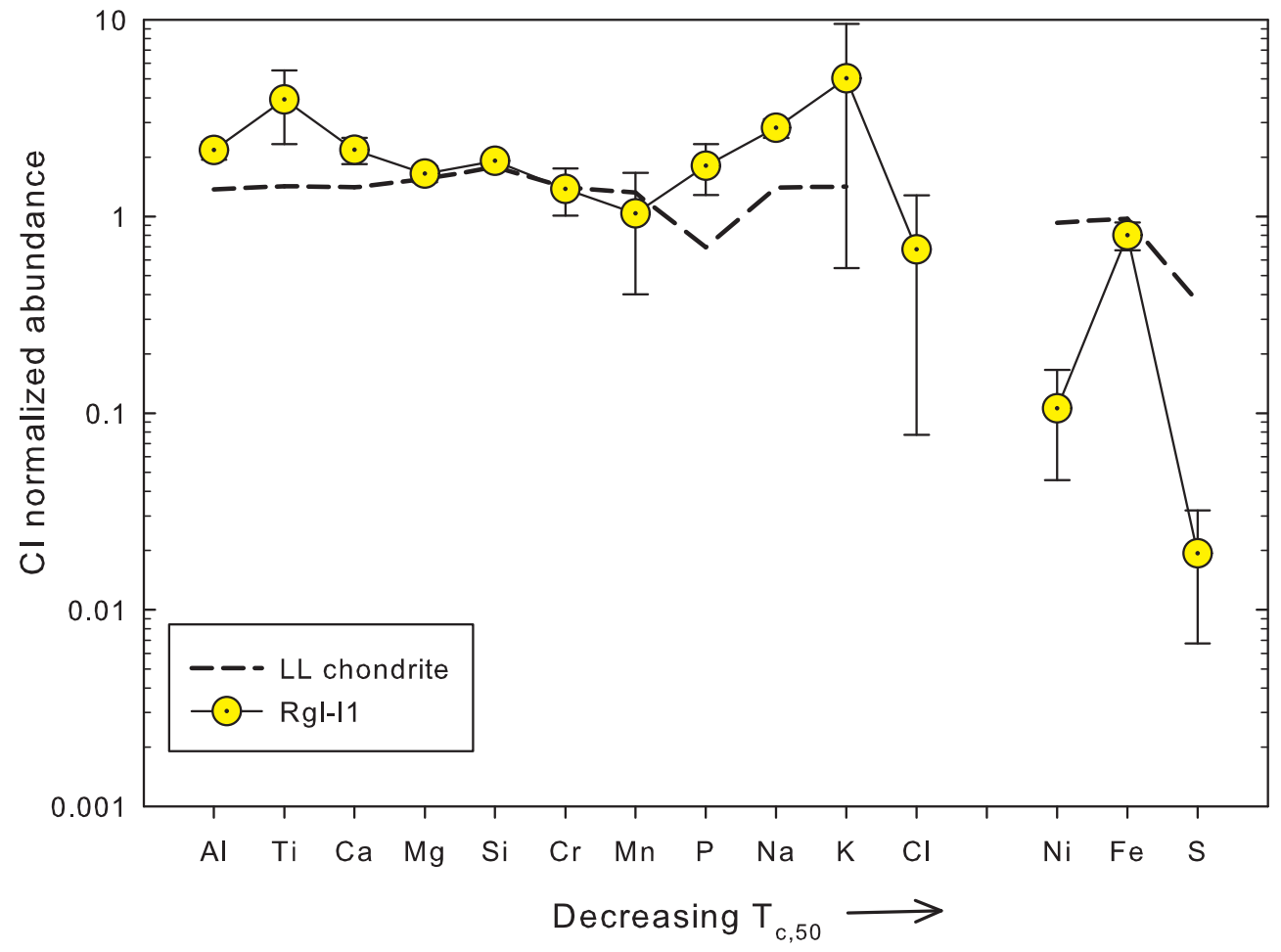

Figure A.40: CI-normalized bulk chemistry of Rgl-I1. This inclusion is largely chondritic, with an enrichment in the alkali elements that could be result of metasomatism. The $\mathrm{K}$ enrichment may also be a result of shock (see section 4.2.1). 
A Chemical and petrographic descriptions of individual inclusions

\section{A.18 Parnallee (CML 0524) LL3.6}

Parnallee is an LL3.6 chondrite. Two thin sections containing three inclusions were examined. Prn-1 and Prn-2 are in close proximity to each other $(\sim 1 \mathrm{~mm})$ in one thin section, Prn-3 is in a separate PTS.

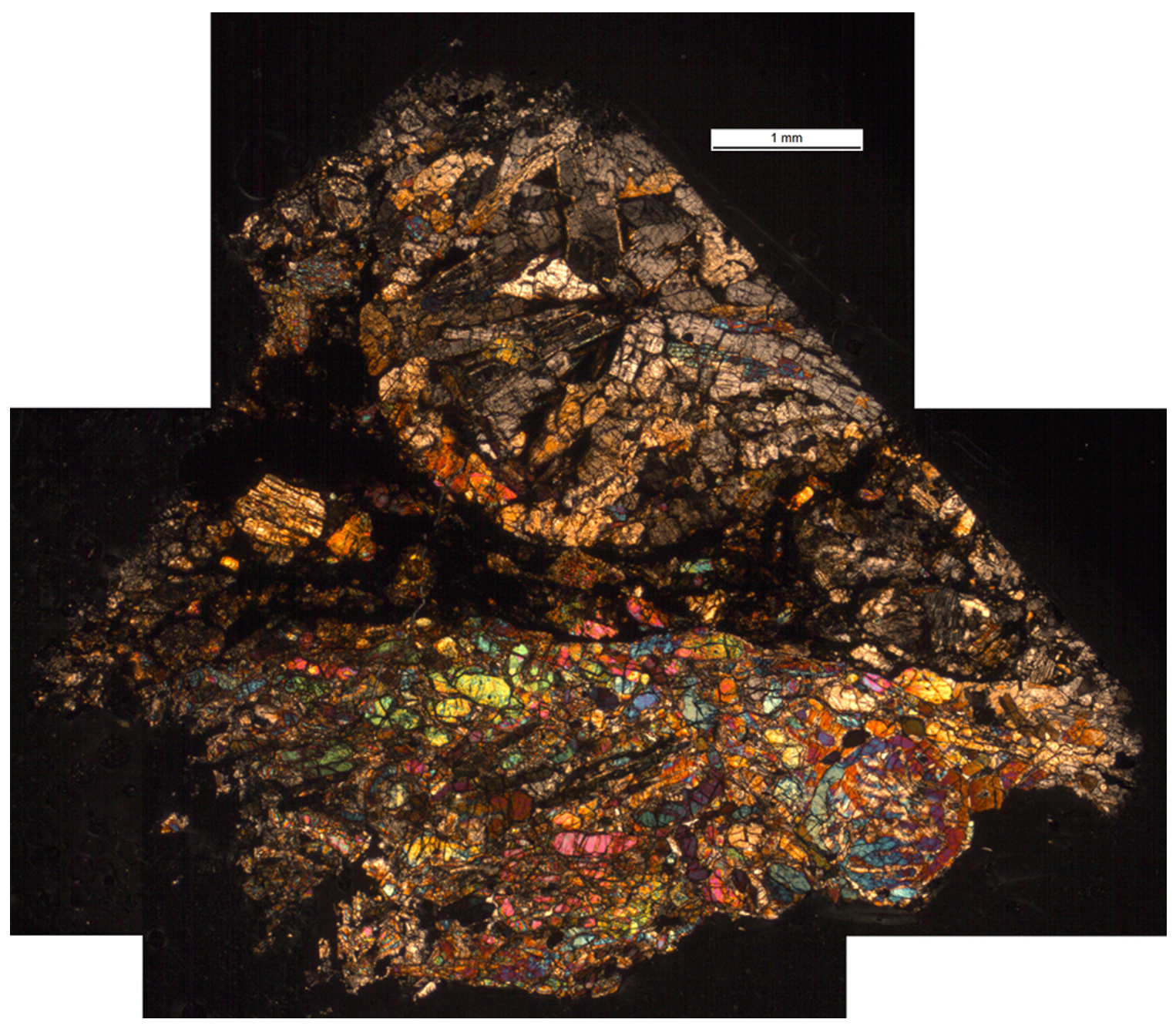

Figure A.41: Cross-polarized light micrograph of Par-I1 (bottom) and Par-I2 (top). 
A Chemical and petrographic descriptions of individual inclusions

Par-I1 Only a small fragment of this inclusion is available, but based its curved contact with the host, it could have an original diameter of $>1 \mathrm{~cm}$. The inclusion has a sharp contact with the host, and many crystals at the contact appear broken. Close to the contact, there are at least two fracture zones within the inclusion where the it has been sheared and offset relative to itself. At the contact, the inclusion is rimmed by a band of low-Ca pyroxene that is more magnesian than the low-Ca pyroxene in the interior.

Mineralogically, the inclusion is dominated by coarse $(100-500 \mu \mathrm{m})$, subhedral olivine that is unequilibrated and has a typical zoning pattern of magnesian core and ferroan rim. Intergrown with the olivine and in a few grains poikilitically enclosing olivine is subhedral to anhedral low-Ca pyroxene, which often has a high-Ca pyroxene overgrowth. Anhedral feldspar is interstitial to the olivine and pyroxene, and minute grains of ilmenite, chromite, and FeNi metal are throughout the inclusion. Sulfide is almost entirely absent, but there are a couple small grains close to the host contact.

The olivine has average composition of Fa $21.9 \pm 2.0(\mathrm{~N}=24)$. The majority of the pyroxene in the interior of the inclusion is pigeonite, with composition Wo $10.1 \pm$ 1.7, Fs $19.5 \pm 2.4$, En $70.4 \pm 3.5(\mathrm{~N}=8)$, though some of the grains and the low-Ca pyroxene at the rim of the inclusion have composition Wo $5.9 \pm 0.7$, Fs $16.7 \pm 0.8$, En $77.4 \pm 1.2(\mathrm{~N}=8)$. This and the olivine has a low $\mathrm{FeO}$ content for an LL chondrite. The pigeonite is often overgrown with diopside with composition Wo $35.8 \pm 2.2$, Fs $11.6 \pm 0.7$, En $52.7 \pm 1.5(\mathrm{~N}=11)$. The feldspar is very calcic, with composition $\mathrm{Ab}$ $14.0 \pm 2.2$, Or $0.2 \pm 0.1$, An $85.8 \pm 2.1(\mathrm{~N}=12)$. Olivine and feldspar compositions were determined with EDS point spectra. Both pyroxenes, ilmenite, chromite, and metal compositions were determined with EDS map extracts and AZtec's analyze 
A Chemical and petrographic descriptions of individual inclusions

phases.

There is at least one olivine "donut" (see section 4.4), $1.1 \mathrm{~mm}$ in apparent diameter. A circular rim of optically aligned olivine contains an olivine-pyroxene-feldspar assemblage, the olivine of which is optically aligned with the rim.

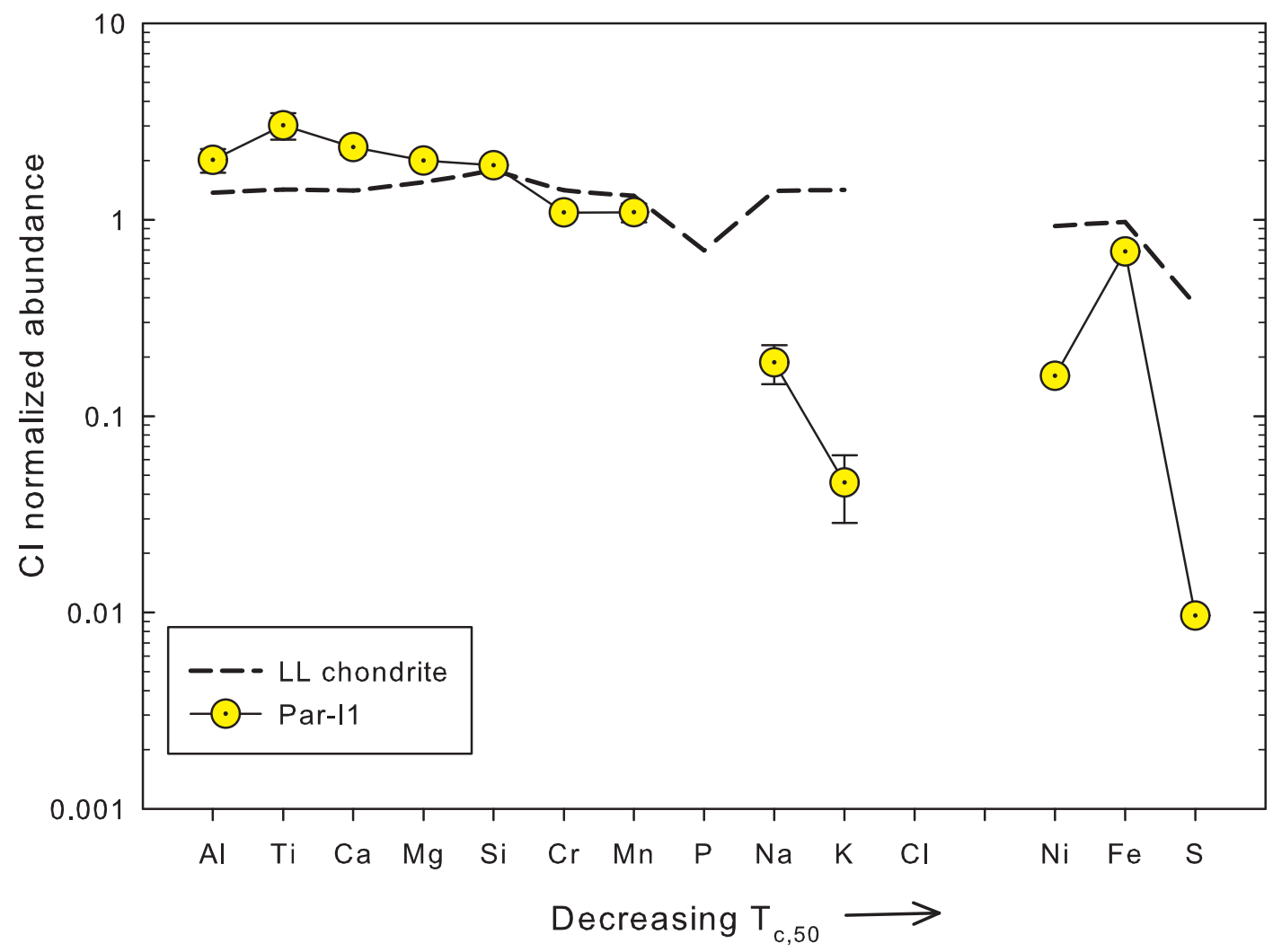

Figure A.42: CI-normalized bulk chemistry of Par-I1. This inclusion is depleted in volatiles, suggesting a vapor fractionation process.

Par-I2 This droplet-formed inclusion is also cut off, but a majority of it is available in this thin section. It is round, with an apparent diameter of $>3 \mathrm{~mm}$. It is in sharp contact with the host, but only a couple crystals appear as though they may have 
A Chemical and petrographic descriptions of individual inclusions

been broken; the majority appear whole. There is a radial variation in texture, with the center dominated by radially aligned, elongate subhedral low-Ca pyroxene grains. These grains are coarse, $\sim 1 \mathrm{~mm}$ long, zoned, and have a clinoenstatite structure. These grains have a thin overgrowth of augite, and are set in a mesostasis of feldspar and calcium-rich pyroxene microlites. Coarse, zoned anhedral olivine is intergrown with the pyroxene further from the center of the inclusion. The edge is dominated by rounded, subhedral low-Ca pyroxene. Minute blebs of metal, chromite, and sulfide are throughout the inclusion, as well as a couple coarser grains of a metal/sulfide assemblage.

The olivine and low-Ca pyroxene are unequilibrated, with average composition Fa $24.5 \pm 3.4(\mathrm{~N}=13)$. and Wo $0.89 \pm 0.6$, Fs $18.4 \pm 3.9$, En $80.7 \pm 4.4(\mathrm{~N}=24)$ respectively, both of which are low in FeO content for an LL chondrite. The augite is variable in composition, with Wo $27.0 \pm 3.6$, Fs $34.9 \pm 4.9$, En $38.1 \pm 7.0(\mathrm{~N}=9)$. All phases were characterized with EDS map extracts. 
A Chemical and petrographic descriptions of individual inclusions

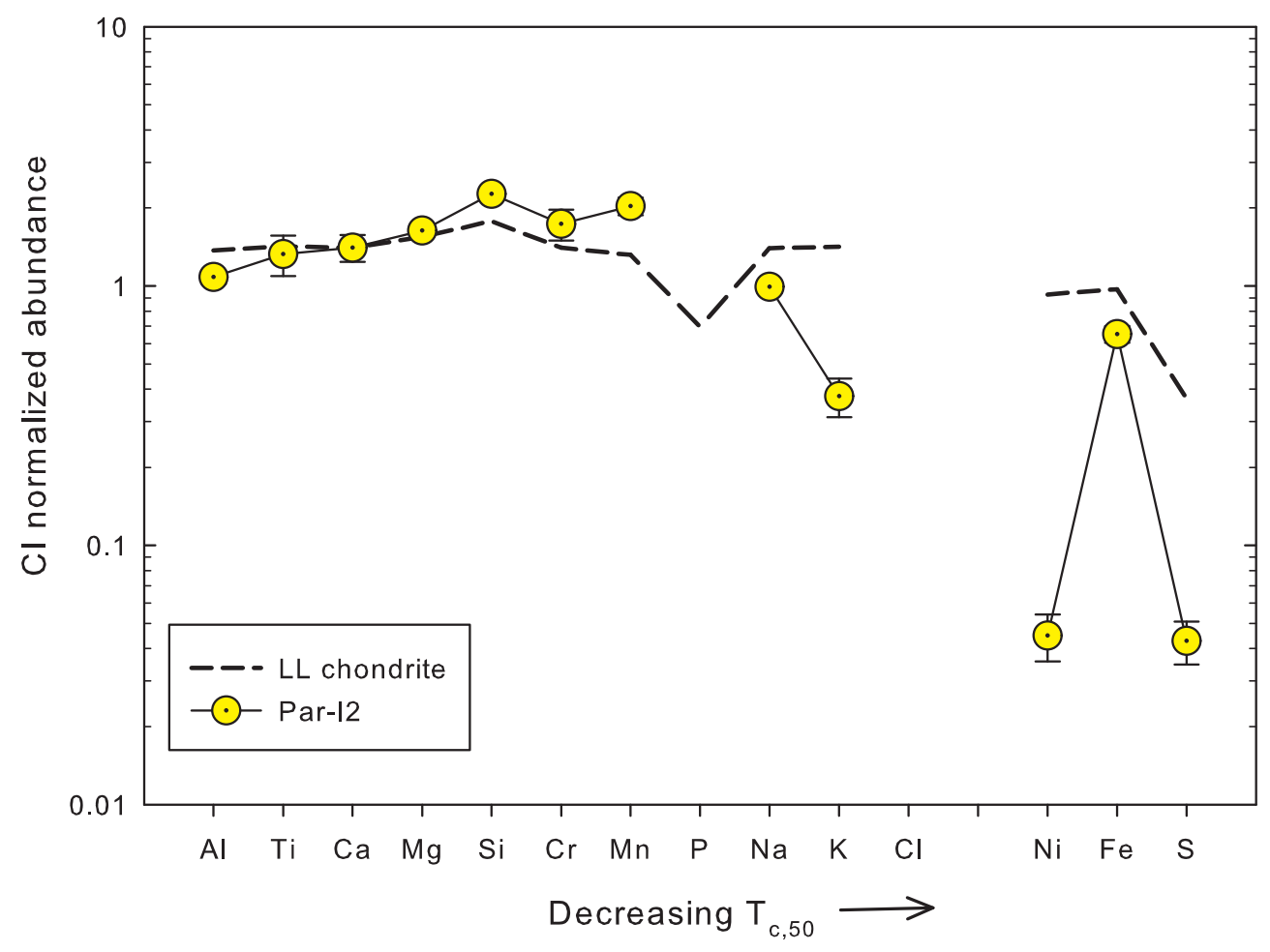

Figure A.43: CI-normalized bulk chemistry of Par-I2. This inclusion is enriched in the moderately volatile elements, suggesting a condensation process. 
A Chemical and petrographic descriptions of individual inclusions

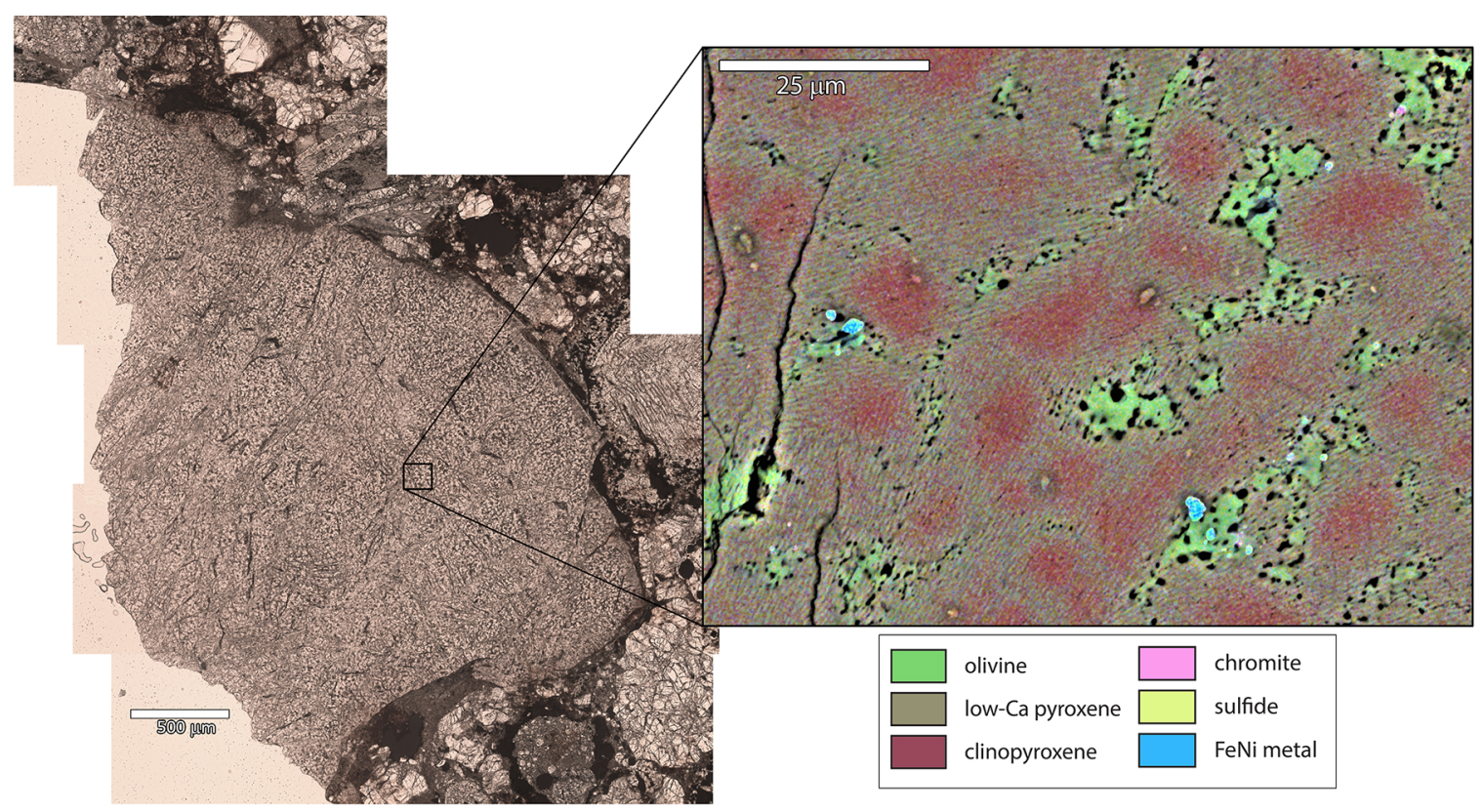

Figure A.44: Transmitted light micrograph of Par-I3, with false-color EDS map zoom-in.

Par-I3 This droplet-formed inclusion is very fine grained. It has a sharp contact with host, and it appears to be cut by a number of fracture zones that offset the inclusion edge, but do not continue into the host. It has a $3.3 \mathrm{~mm}$ apparent diameter, though it extends to the edge of the specimen and may be larger. It is largely very fine grained pyroxene, with pockets of Ca-rich pyroxene mixed into low-Ca pyroxene. Olivine is interstitial. The pyroxene, both calcium-rich and calcium-poor, occurs as sets of mutually aligned needles or platy crystals, $<1 \mu \mathrm{m}$ across, but extending tens of microns in length. Minute $(<10 \mu \mathrm{m})$ blebs of FeNi metal and chromite are throughout the inclusion.

All phases are equilibrated, which must have occurred before the inclusion was incorporated into the host. The olivine has average composition of Fa $29.5 \pm 0.3(\mathrm{~N}$ =4). The low-Ca pyroxene averages Wo $3.8 \pm 0.6$, Fs $25.8 \pm 0.2$, En $70.4 \pm 0.6$ 
A Chemical and petrographic descriptions of individual inclusions

( $\mathrm{N}=7$ map extracts), with the calcium-rich areas average Wo $12.7 \pm 0.5$, Fs 25.8 \pm 0.2 , En $70.4 \pm 0.6(\mathrm{~N}=4$ map extracts $)$. The FeO content of the olivine and low-Ca pyroxene are average for an LL chondrite. Olivine, metal, and chromite were characterized with EDS point spectra; other phases were characterized with EDS map extracts.

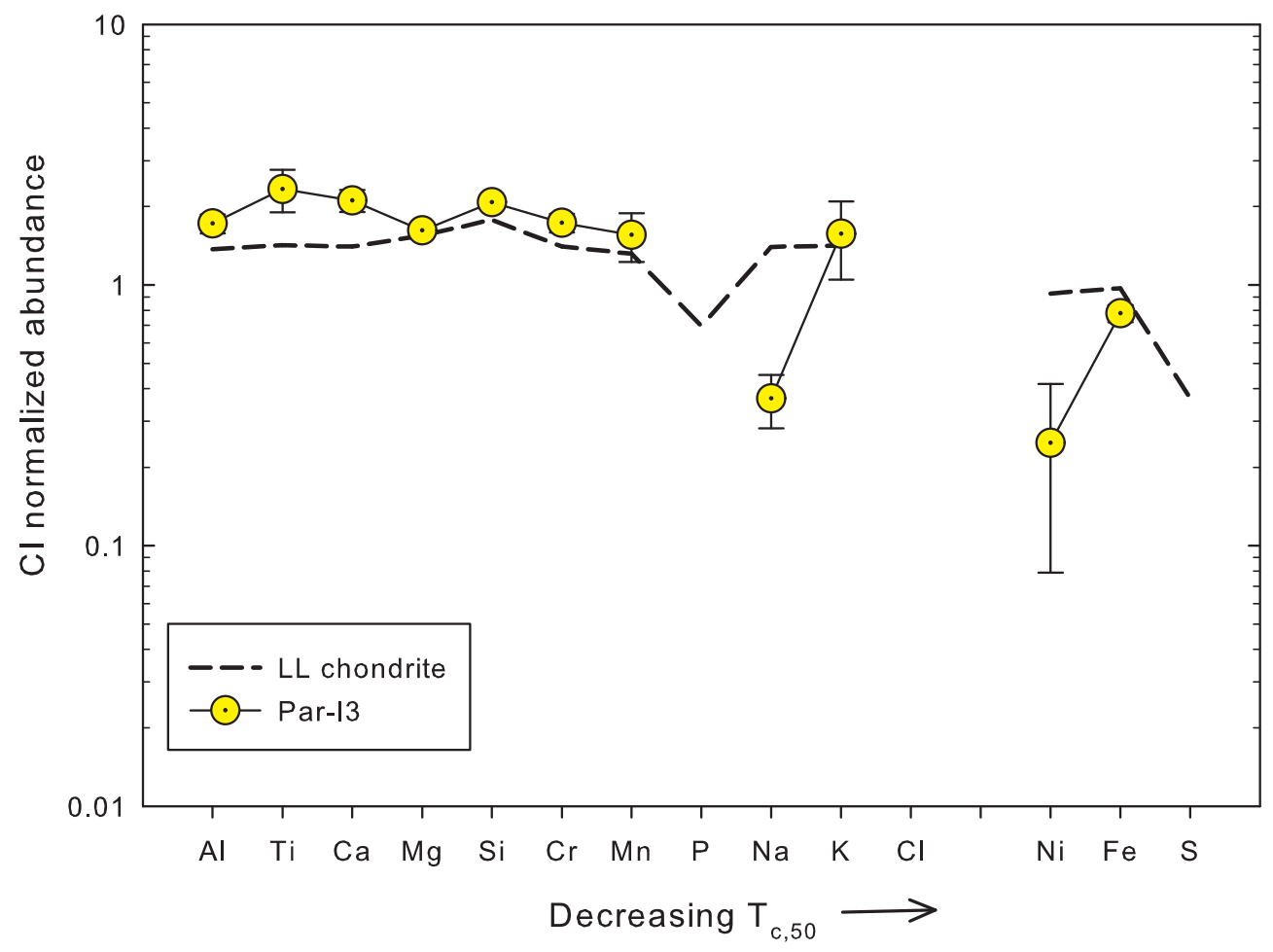

Figure A.45: CI-normalized bulk chemistry of Par-I3. This inclusion is essentially chondritic, though except for $\mathrm{Mg}$, may also be enriched in the elements of mid-volatility.

\section{A.19 Lut 005 (CML 0740) LL3.7}

Lut 005 is an LL3.7 chondrite. One inclusion from this meteorite was examined. 
A Chemical and petrographic descriptions of individual inclusions

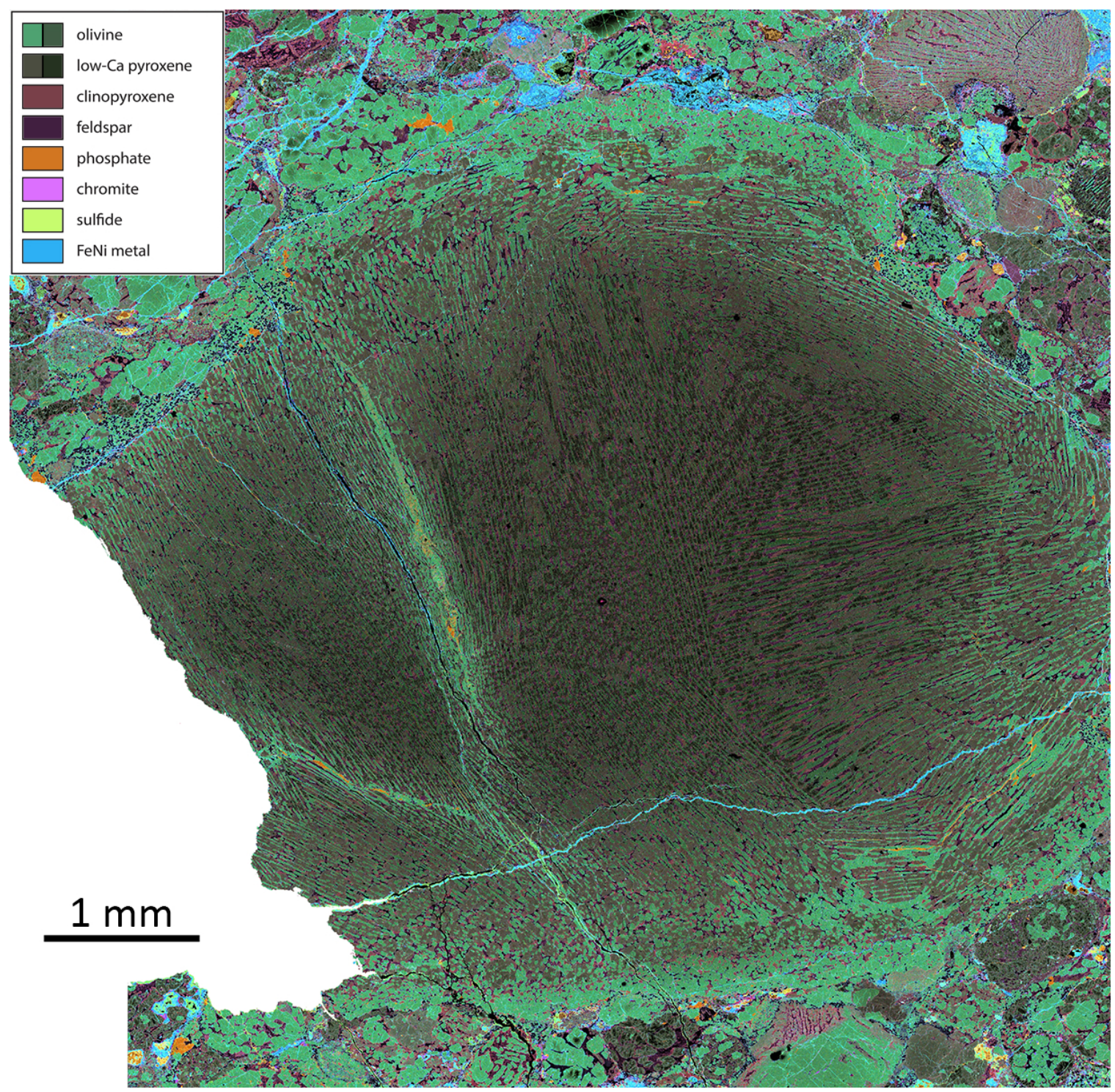

Figure A.46: False-color EDS map of Lut-I1.

Lut-I1 This inclusion is rounded in shape and radially variable in texture, similar to the inclusion in NWA 7870 (0173). It extends to the broken edge of the specimen, and so is greater than $8 \mathrm{~mm}$ in maximum diameter. At the host contact, the edge of the inclusion is characterized by a rim of porphyritic olivine with interstitial high-Ca 
A Chemical and petrographic descriptions of individual inclusions

pyroxene and feldspar, indicating that this is an original edge, and that the inclusion was incorporated into the host as a whole, previously formed object.

Like 7870-I1, this specimen has zones of mutually aligned skeletal olivine intergrown with low-Ca pyroxene, though in this case the low-Ca pyroxene is unequilibrated and there are two distinct phases. High-Ca pyroxene, feldspar, and feldspathoid are interstitial. There is a minor amount of phosphate, and the inclusion is nearly completely depleted in metal and sulfide; all present is filling fractures and could have mobilized from the host, which is significantly weathered.

There is a significant radial variation in olivine and pyroxene chemistry from the core to the rim of the inclusion, with more magnesian phases in the interior and more ferroan phases towards the rim. The interstitial feldspathic phases vary also, with only plagioclase in the center and intergrown plagioclase and sodalite at the edge. This, in addition to the radial textural differences, suggests that this inclusion solidified as a free-floating droplet.

Olivine composition is Fa $19.9 \pm 6.3(\mathrm{~N}=12)$, low-Ca pyroxene is Wo $3.3 \pm 1.8$, Fs $10.9 \pm 2.4$, En $85.8 \pm 3.6(\mathrm{~N}=114)$. Clinopyroxene is even more variable, with Wo $23.7 \pm 11.7$, Fs $10.1 \pm 2.9$, En $66.3 \pm 11.1(\mathrm{~N}=15)$. All phases were characterized with EDS point spectra. 
A Chemical and petrographic descriptions of individual inclusions

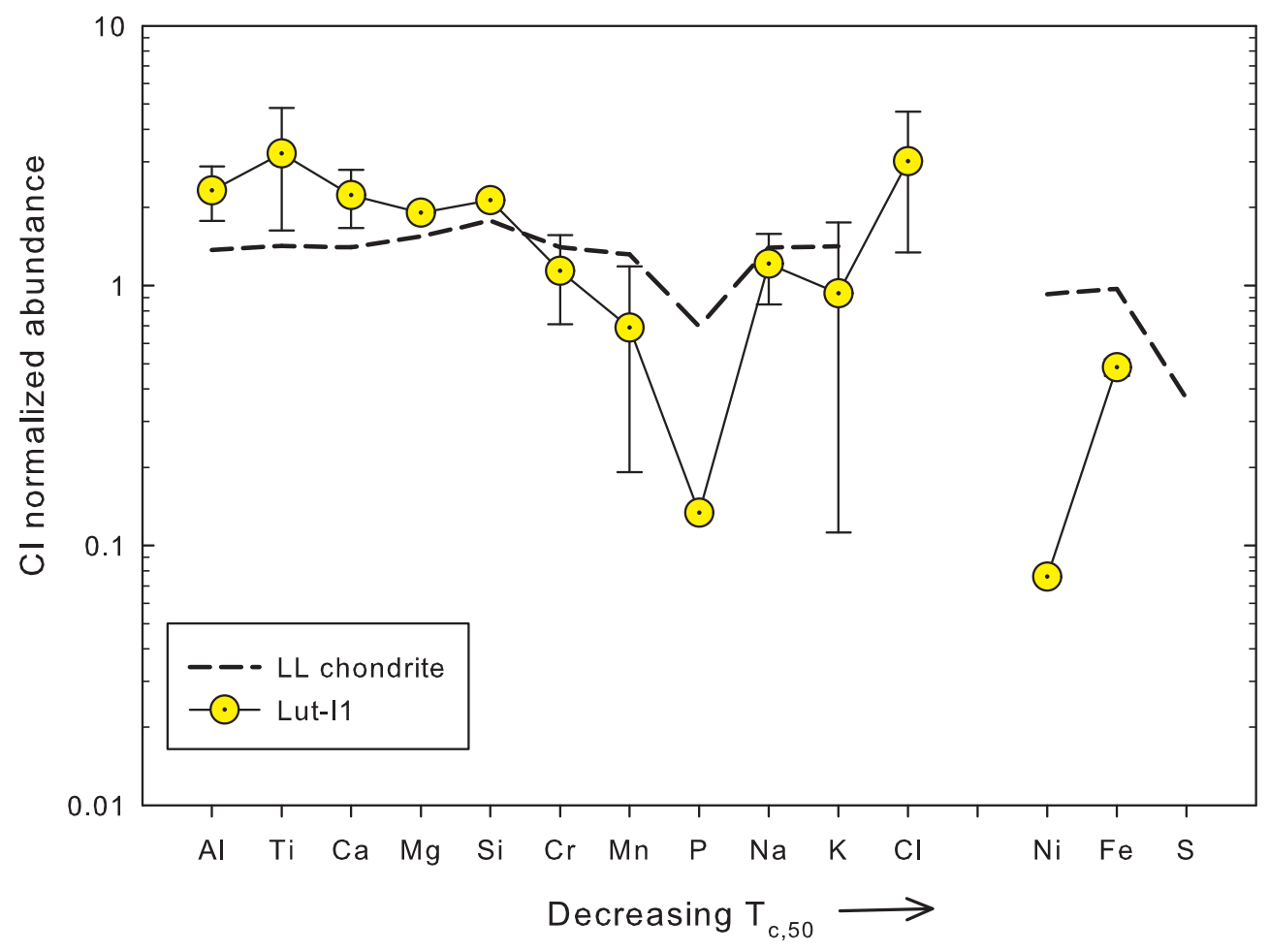

Figure A.47: CI-normalized bulk chemistry of Lut-I1. This inclusion likely was vapor fractionated, with later metasomatism.

\section{A.20 Richfield (AMNH 4887) LL3.7}

Richfield is a LL3.7 chondrite. One inclusion in one polished thick section was examined with reflected light optical microscopy and SEM. 
A Chemical and petrographic descriptions of individual inclusions

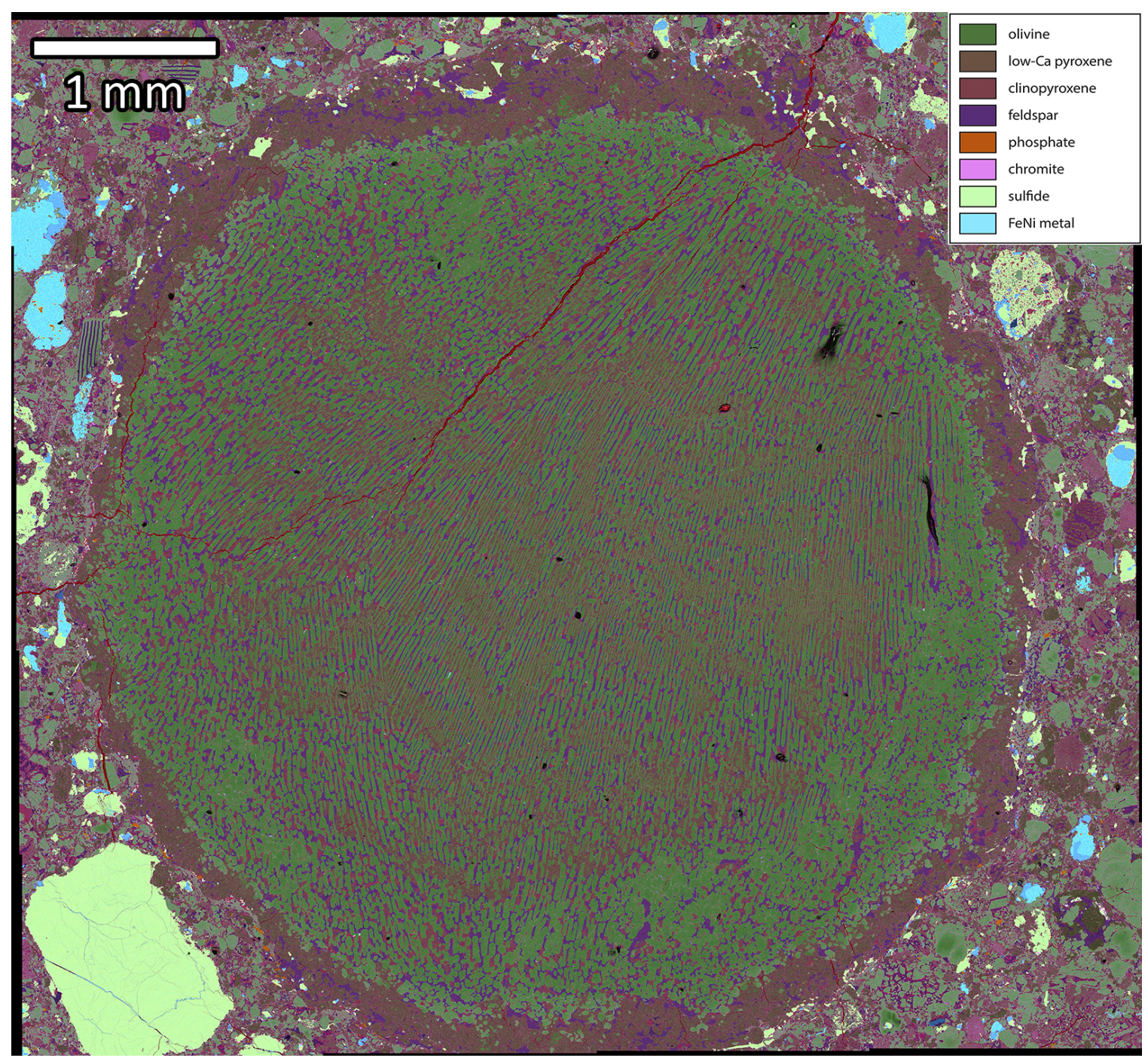

Figure A.48: False-color EDS phase map of Rfd-I1.

Rfd-I1 This inclusion is round, approximately 5 - $5.5 \mathrm{~mm}$ in apparent diameter. Its contact with the host is not smooth, but is clear and easily discernible. It is radially variable in mineralogy, chemistry, and texture. Most obvious is a continuous rim of coarse, anhedral low-Ca pyroxene several hundred microns thick. The radial variability and existence of a rim suggest this is not a fragment of a larger object, 
A Chemical and petrographic descriptions of individual inclusions

and that it was incorporated into the host as a whole object.

Texturally, it, like many other inclusions in this study, resembles a barred olivine chondrule. Elongate, mutually aligned olivine crystals are intergrown with low-Ca pyroxene, with diopside and feldspar interstitial. Like inclusion 7870-I1, it towards the rim it has a more porphyritic texture, and here the low-Ca pyroxene is absent, appearing again as the outer rim. Very close to the pyroxene rim, the olivine of the inclusion is more fayalitic than elsewhere in the inclusion. Very minor amounts of chromite, metal, sulfide, and ilmentite, more metal and sulfide closer to contact.

Olivine is unequilibrated, largely due to the fayalitic rim rather than zoning, with average composition Fa $18.3 \pm 3.2(\mathrm{~N}=30)$. The low-Ca pyroxene is equilibrated, with average composition Wo $2.1 \pm 0.5$, Fs $11.1 \pm 1.2$, En $86.8 \pm 1.6(\mathrm{~N}=24)$. The diopside is more variable in composition, with average composition Wo $33.7 \pm 5.6$, Fs $10.4 \pm 1.3$, En $56.0 \pm 4.4(\mathrm{~N}=9)$. The feldspar is anorthitic, with composition Ab $15.8 \pm 1.3$, Or $0.5 \pm 0.2$, An $83.7 \pm 1.4(\mathrm{~N}=16)$. Olivine, low-Ca pyroxene, diopside, feldspar, chromite, and ilmenite were characterized with EDS point spectra. Metal and sulfide compositions were determined from EDS map extracts. 
A Chemical and petrographic descriptions of individual inclusions

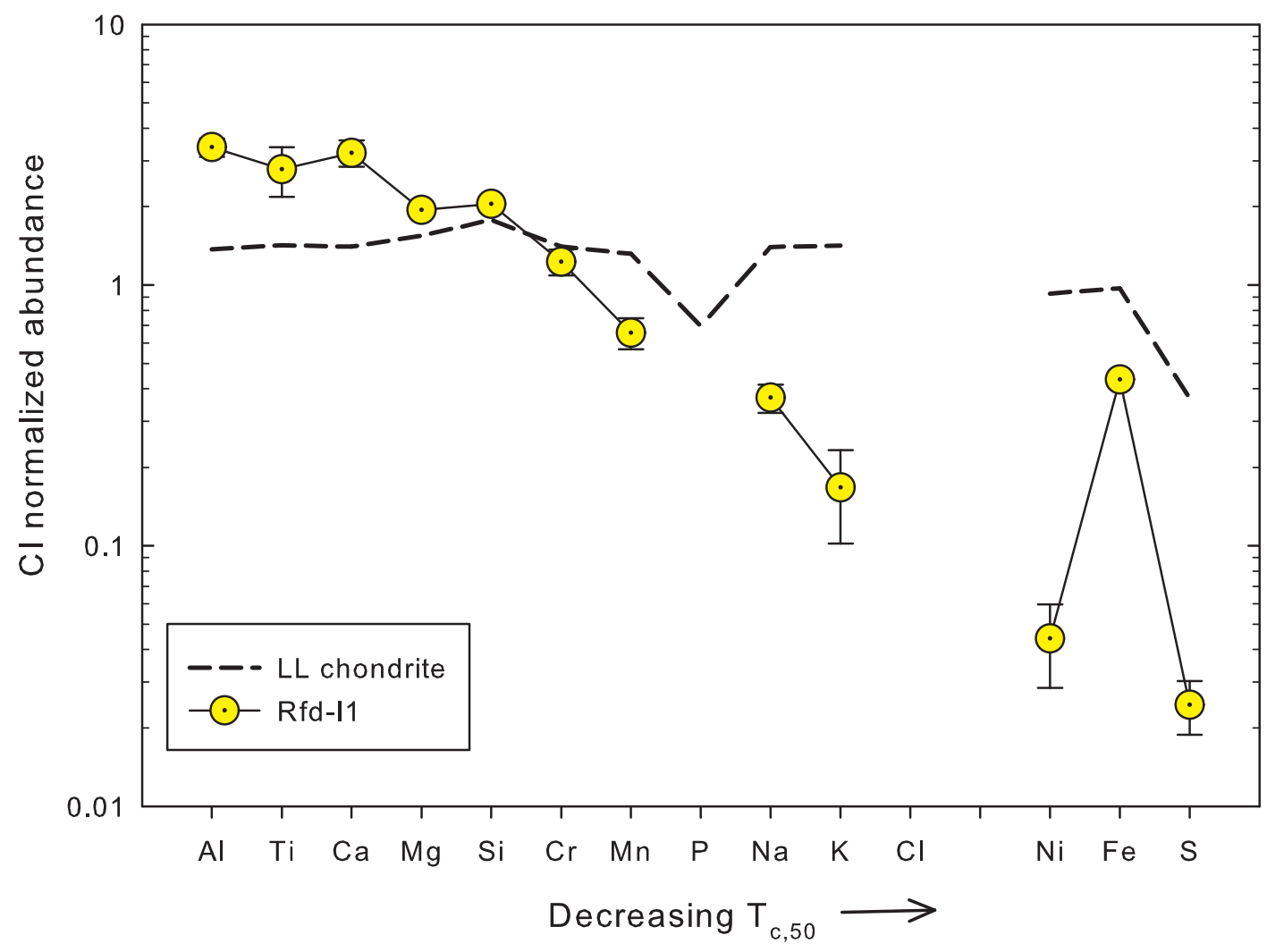

Figure A.49: CI-normalized bulk chemistry of Rfd-I1. This inclusion is enriched in refractory elements and depleted in volatiles, suggesting a vapor fractionation process.

\section{A.21 NWA 4859 (CML 0272) LL5}

This meteorite, an LL5, contains a large number of inclusions, many of which were described by Jamsja and Ruzicka (2010). Two inclusions in two polished thin sections are described here. 
A Chemical and petrographic descriptions of individual inclusions

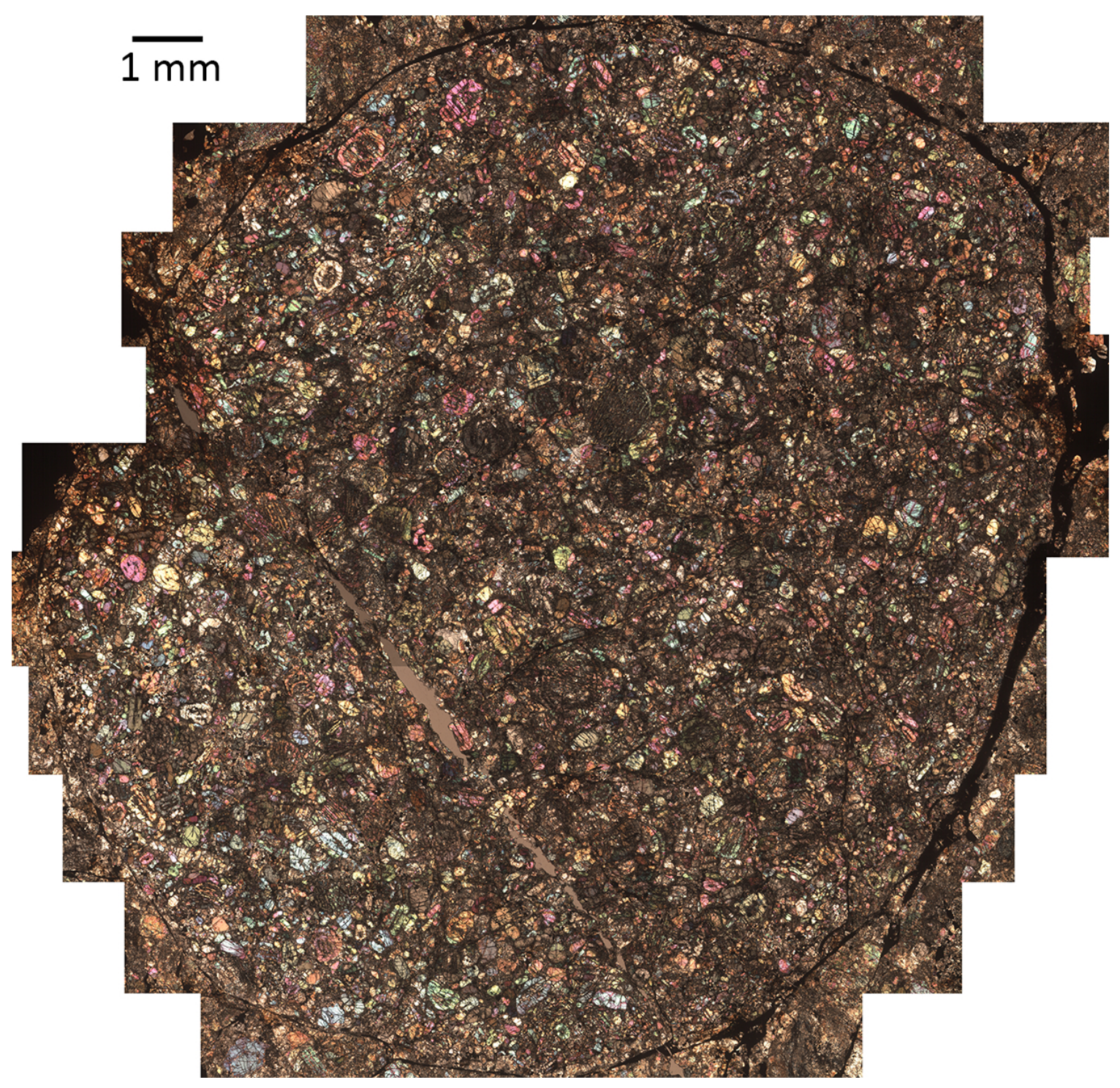

Figure A.50: 4859-I4 under cross-polarized light.

NWA 4859-I4 This inclusion (LITE 4 in Jamsja and Ruzicka, 2010) is large, with an apparent diameter of $16 \mathrm{~mm}$, and rounded. The contact with the host is almost entirely defined by a rim of low-Ca pyroxene, outside of which is a clear shock vein. There are some crystals at the contact that appear truncated, suggesting some 
A Chemical and petrographic descriptions of individual inclusions

brecciation, but the almost-complete pyroxene rim suggests that this was a discrete object that was interacting with its surroundings. There are several fractures crossing the inclusion that do not continue into the host, suggesting some brecciation occurred before the inclusion was incorporated into the host.

The texture can probably be best described as an olivine-pyroxene microporphyry, with anhedral to subhedral olivine, low-Ca pyroxene, and diopside phenocrysts in a feldspathic groundmass. A large fraction of the olivine, however, is present as large ( $100-1000 \mu \mathrm{m}$ ), circular "shells" (olivine "donuts," see section 4.4) This inclusion is the only one in this study to contain a large number $(>3)$ of these objects. Minor phases include minute $(\leq 10 \mu \mathrm{m})$ chromite, sulfide and FeNi metal blebs the inclusion, and coarser, anhedral $(\sim 100 \mu \mathrm{m})$ apatite phosphate.

The olivine is equilibrated, with average composition of Fa $28.4 \pm 0.1(\mathrm{~N}=34)$. Low-Ca pyroxene and diopside are also equilibrated, with compositions Wo $1.7 \pm 0.3$, Fs $19.2 \pm 0.3$, En $79.0 \pm 0.4$, and Wo $41.7 \pm 0.3$, Fs $7.5 \pm 0.2$, En $50.8 \pm 0.3,(\mathrm{~N}=8$, 16) respectively. Feldspar is more variable in composition, with $\mathrm{Ab} 81.6 \pm 1.5$, Or 3.7 \pm 0.7 , An $14.7 \pm 2.0(\mathrm{~N}=9)$. Olivine composition was determined with EMPA and EDS point spectra. Low-Ca pyroxene, feldspar, phosphate, metal and sulfide were characterized with EDS map extracts. Chromite and ilmenite were characterized with AZtec's analyze phases. 
A Chemical and petrographic descriptions of individual inclusions

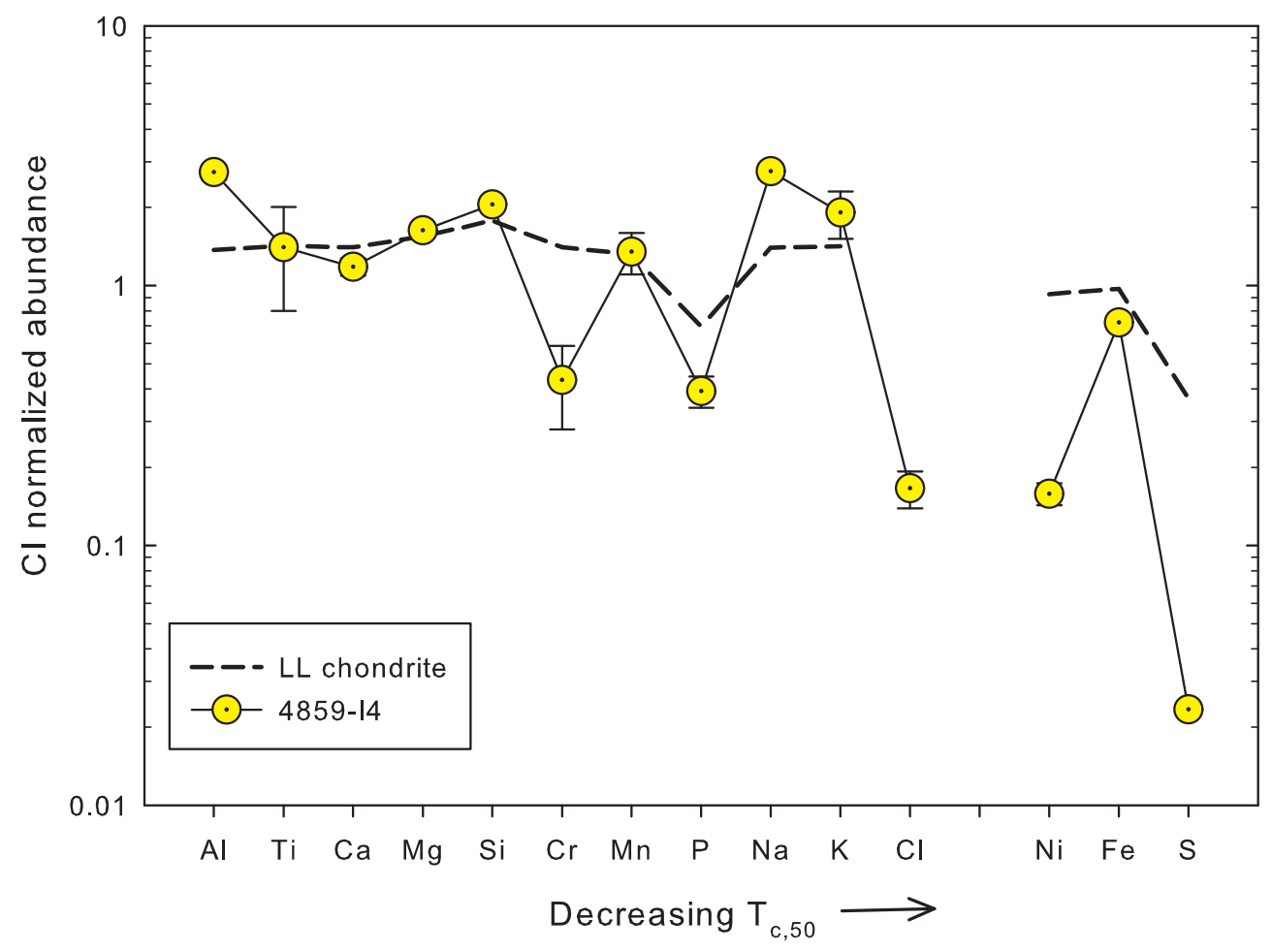

Figure A.51: CI-normalized bulk chemistry of 4859-I4. This inclusion is essentially chondritic, though enriched in the elements that form feldspar and depleted in $\mathrm{Cr}$ (see section $4.3 .2)$. 
A Chemical and petrographic descriptions of individual inclusions

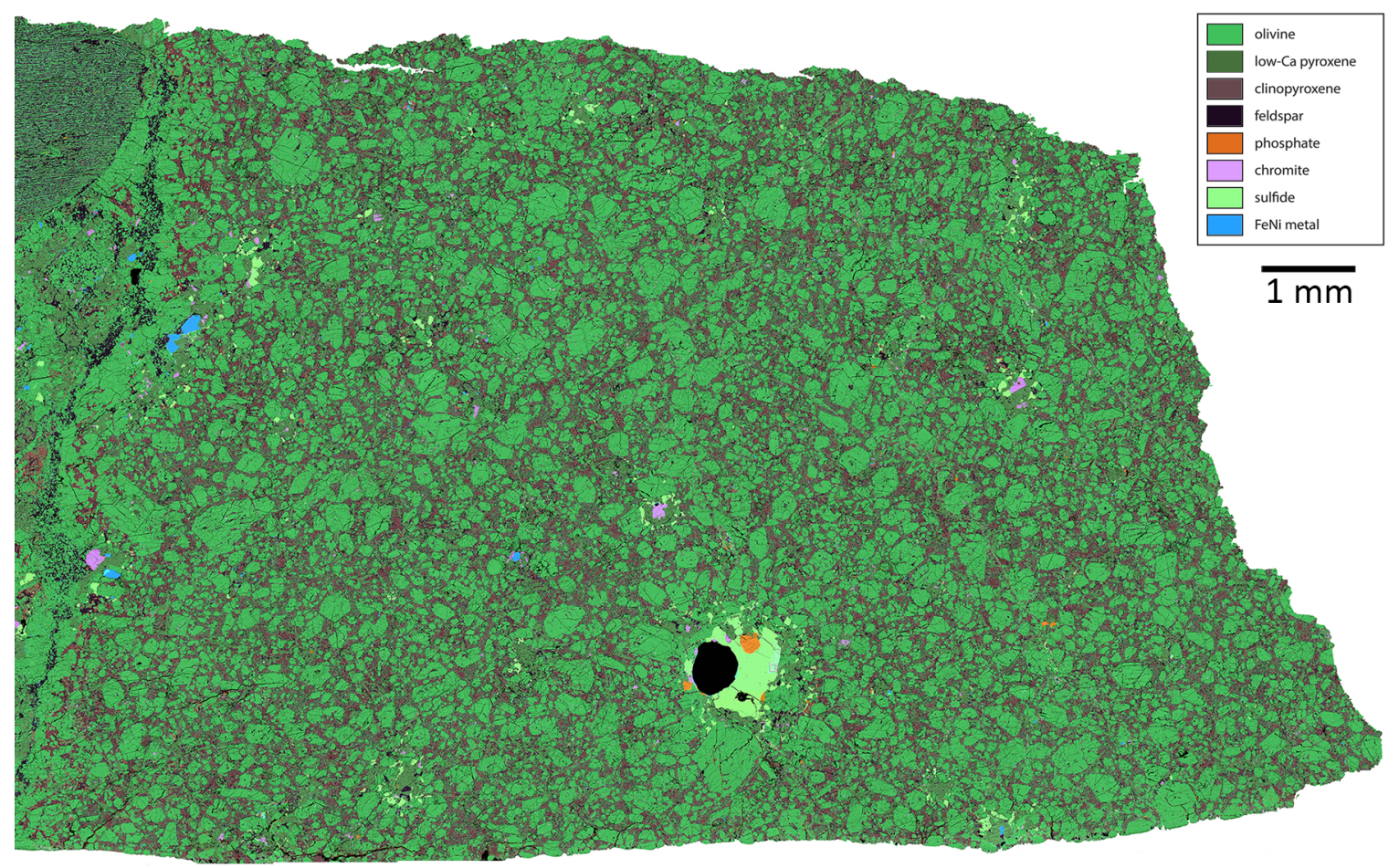

Figure A.52: False-color EDS phase map of 4859-I18.

NWA 4859-I18 This PTS contains one large inclusion. It is impossible to tell its original size, as this inclusion occupies almost the entire slide, but the largest dimension of this specimen is over $18 \mathrm{~mm}$. At the host contact is a nearly unbroken band of coarse olivine, just inside a fine grained mix of olivine and feldspar. Some of the coarse olivine grains appear broken at the contact, indicating that the inclusion may be a broken fragment of a larger object.

It is an olivine microporphyry, with euhedral to subhedral olivine phenocrysts in a groundmass of anhedral low-Ca pyroxene, subhedral, elongate diopside, and anhedral feldspar. The diopside also forms overgrowths on much of the low-Ca pyroxene. Troilite, chromite, taenite, pentlandite, ilmenite and both merrillite and fluorapatite phosphate are present as minor phases. The olivine grain sizes vary from $\sim 50 \mu \mathrm{m}$ 
A Chemical and petrographic descriptions of individual inclusions

to $\sim 500 \mu \mathrm{m}$. Low-Ca pyroxene grains are typically on the order of $\sim 100 \mu \mathrm{m}$, while high-Ca pyroxene and feldspar are on the order of $\sim 10-50 \mu \mathrm{m}$.

The olivine and pyroxene phases are highly equilibrated. Average olivine composition is $\mathrm{Fa} 30.0 \pm 0.1(\mathrm{~N}=15)$. Low-Ca pyroxene has average composition Wo 1.7 \pm 0.4 , Fs $24.6 \pm 0.2$, En $73.6 \pm 0.3(\mathrm{~N}=14)$, and the diopside has composition Wo $47.3 \pm 0.8$, Fs $8.6 \pm 0.5$, En $44.2 \pm 0.9$ (From Analyze Phases). Feldspar is more variable in composition, with average $\mathrm{Ab} 77.5 \pm 5.2$, Or $5.8 \pm 1.3$, An $16.7 \pm 6.1$ $(\mathrm{N}=9)$. All phases were characterized with EDS map extracts, except for diopside, sulfide, metal, phosphate, and ilmenite, which were determined with AZtec's analyze phases. 
A Chemical and petrographic descriptions of individual inclusions

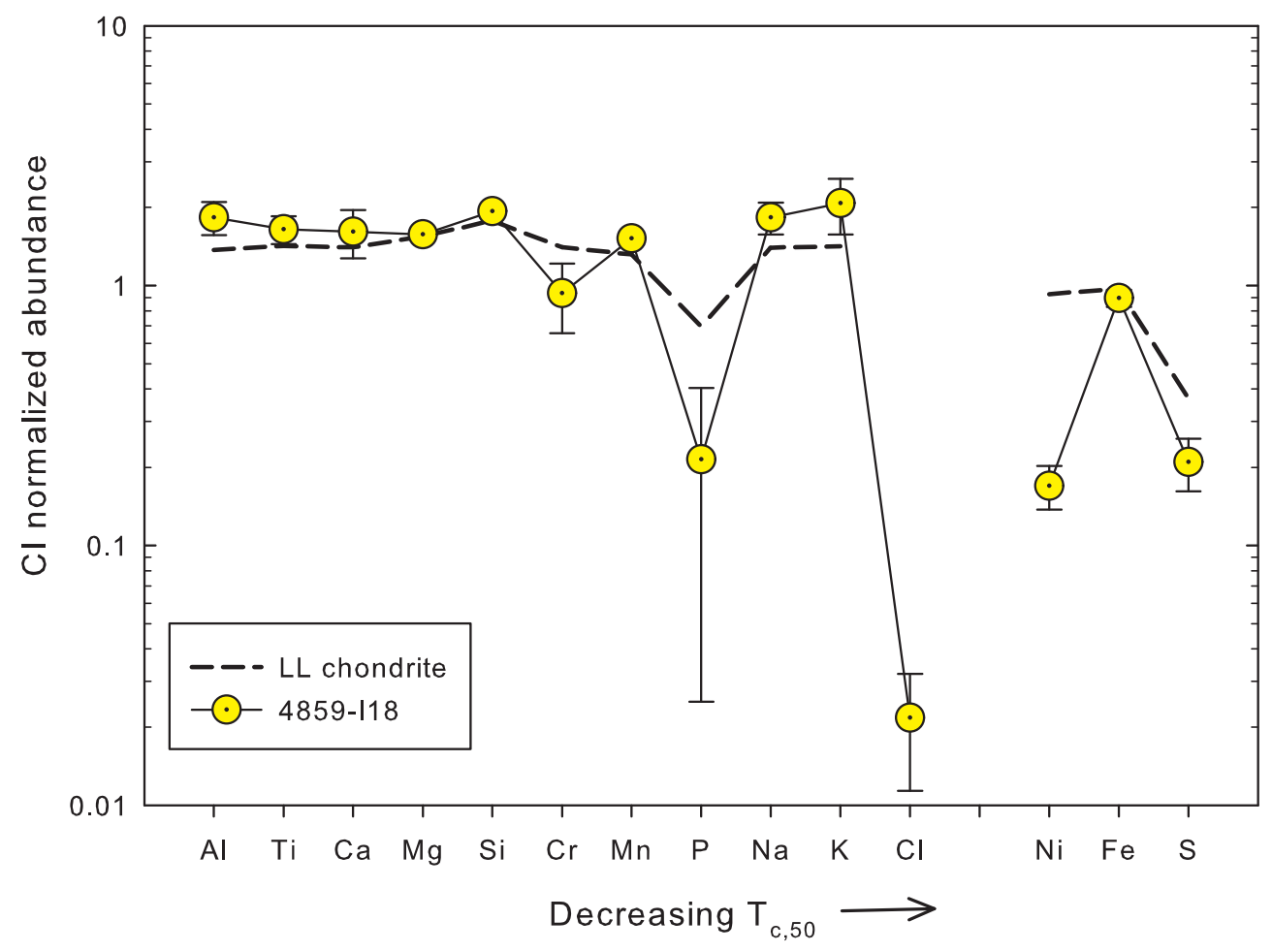

Figure A.53: CI-normalized bulk chemistry of 4859-I18. This inclusion is essentially chondritic.

\section{A.22 NWA 8232 (CML 0372) LL5}

NWA 8232 is an LL5 chondrite. One inclusion in one polished thin section has been examined. 
A Chemical and petrographic descriptions of individual inclusions

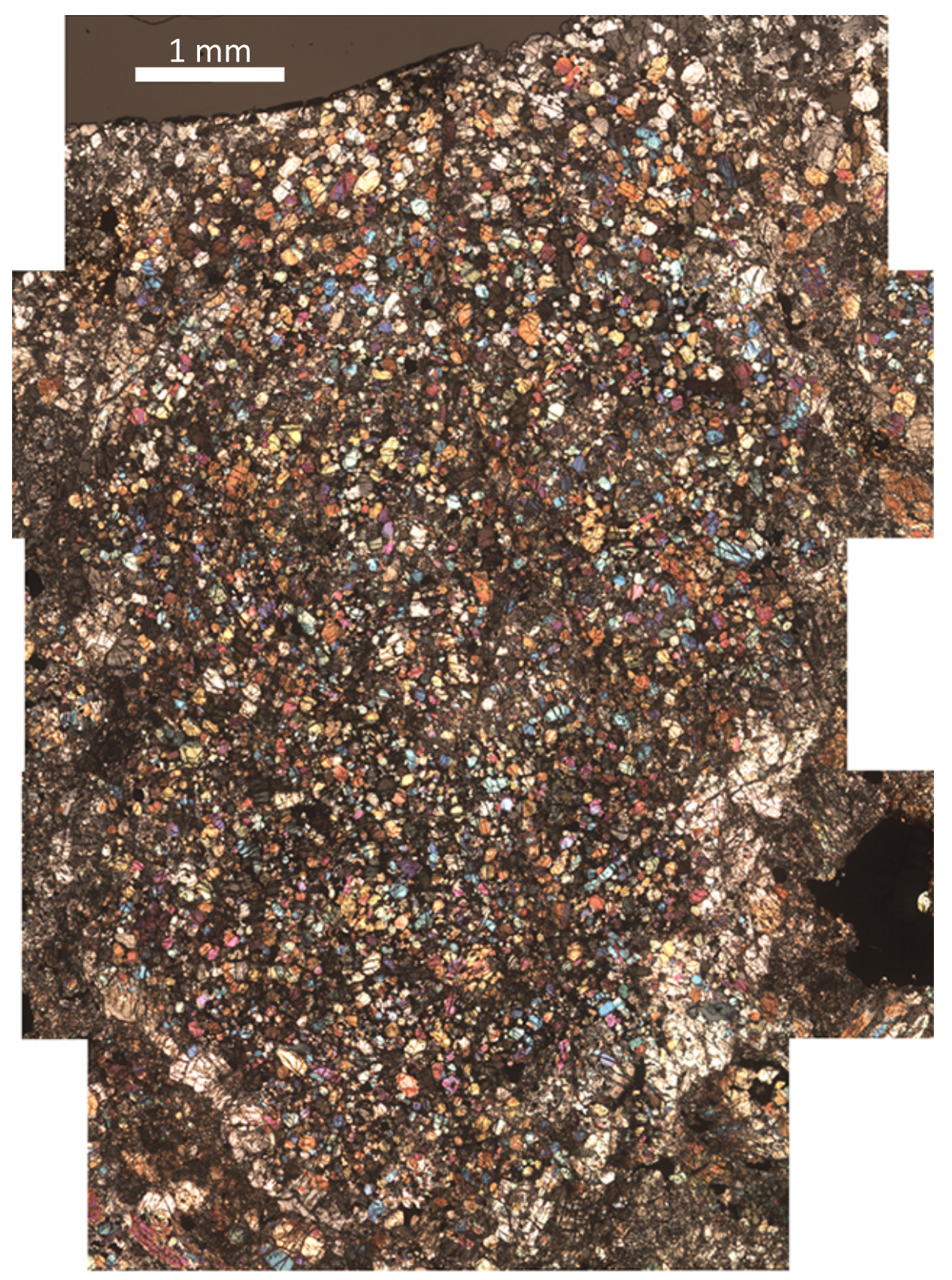

Figure A.54: 8232-I1 under cross-polarized light.

NWA 8232-I1 This is a rounded, oval-shaped inclusion, 8.3 x $5.4 \mathrm{~mm}$ in apparent size. Most of the host contact is sharp and angular, with a nearly complete band of 
A Chemical and petrographic descriptions of individual inclusions

low-Ca pyroxene at the contact. Crystals at the contact do not appear broken.

The majority of this inclusion is an olivine microporphyry. The olivine phenocrysts are subhedral to anhedral, and have a relatively small size distribution; average grain size is $\sim 100 \mu \mathrm{m}$, with a range of $50-150 \mu \mathrm{m}$. There is, however, a secondary population of much smaller olivine $(\sim 10 \mu \mathrm{m})$, that is embayed by a feldspathic groundmass that is interstitial to the phenocrysts. The outer $\sim 0.5 \mathrm{~mm}$ of the inclusion is almost entirely low-Ca pyroxene intergrown with olivine, but there is almost no low-Ca pyroxene in the interior of the inclusion.Diopside is concentrated in small regions at the center of the inclusion, and is mostly absent from the outer 1-2 $\mathrm{mm}$ of the inclusion. The crystals are anhedral, interstitial to the olivine, and $\sim 50-100 \mu \mathrm{m}$ in diameter. Glassy feldspar is interstitial to olivine and pyroxene throughout the inclusion, though is rarer in the outer olivine-low-Ca pyroxene band at the edge. Metal (both kamacite and taenite) and troilite are minor phases, occurring as abundant small $(1-10 \mu \mathrm{m})$, rounded blebs. Chromite occurs as larger $(\sim 50 \mu \mathrm{m})$ grains as well as minute $(<10 \mu \mathrm{m})$ round blebs. Fluorapatite phosphate occurs in fewer, larger grains $(\sim 50 \mu \mathrm{m})$, usually intergrown with the low-Ca pyroxene near the host contact.

The olivine is equilibrated, with average Fa of $29.3 \pm 0.2(\mathrm{~N}=34)$, and low-Ca pyroxene is also equilibrated, with Wo $1.6 \pm 0.2$, Fs $24.2 \pm 0.2$, and En $74.2 \pm$ $0.3(\mathrm{~N}=12)$, both of which are consistent with an LL chondrite. Diopside is also equilibrated, with Wo $45.2 \pm 0.4$, Fs $9.0 \pm 0.4$, and En $45.9 \pm 0.1(\mathrm{~N}=8)$. As the host meteorite is also equilibrated, this equilibration may have happened either before or after the inclusion became incorporated into the host. The feldspathic glass is slightly more variable in composition, with $\mathrm{Ab} 81.1 \pm 1.5$, Or $5.1 \pm 0.7$, and An 13.8 $\pm 1.9(\mathrm{~N}=11)$. Olivine, low-Ca pyroxene, diopside, feldspar, metal, and phosphate 
were characterized with EDS point spectra. Chromite and sulfide compositions were determined with AZtec's analyze phases.

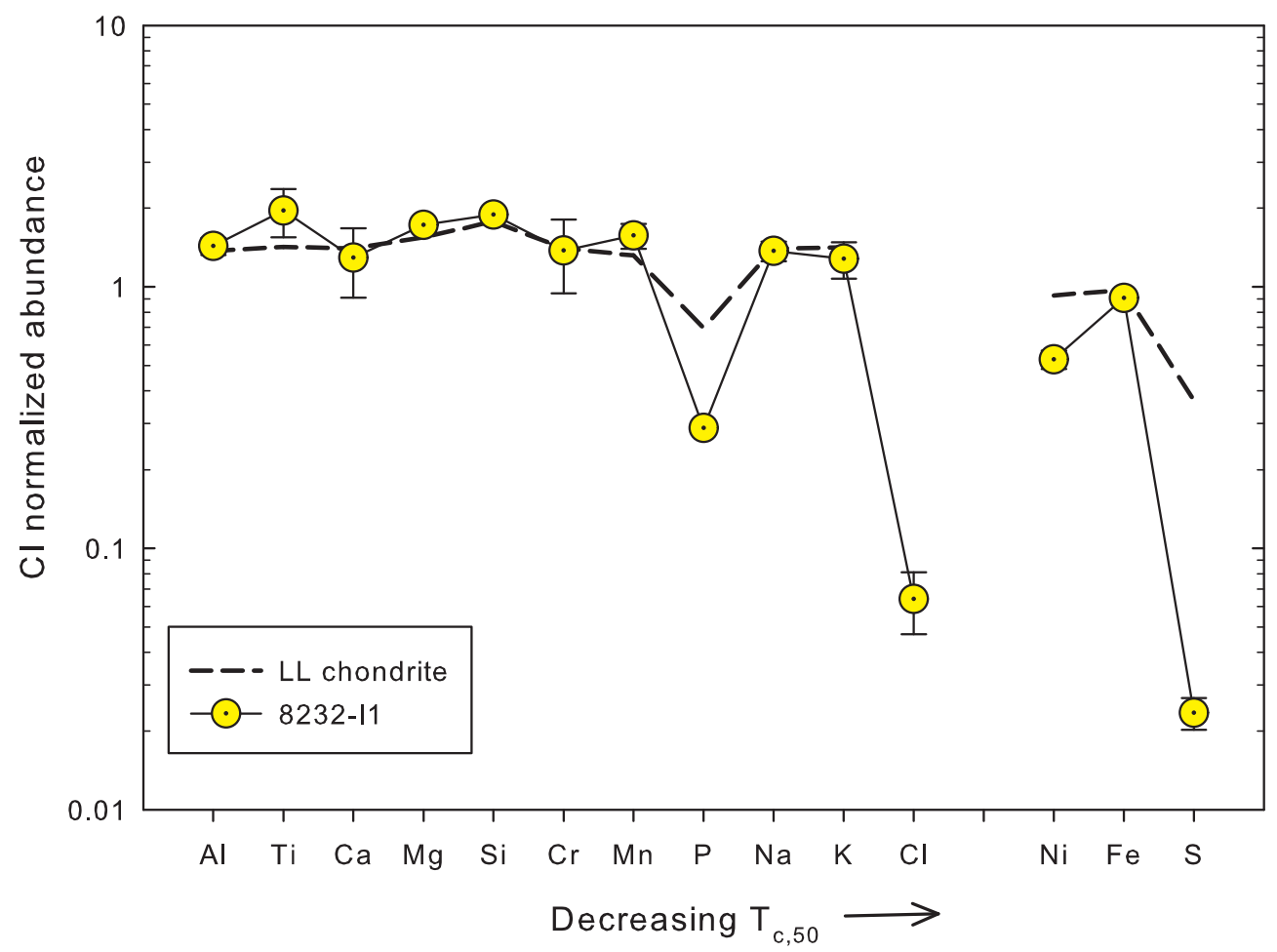

Figure A.55: CI-normalized bulk chemistry of 8232-I1. This inclusion is essentially chondritic.

\section{A.23 Oberlin (AMNH 3832) LL5}

One PTS of Oberlin, an LL5 chondrite, containing one inclusion has been examined. 
A Chemical and petrographic descriptions of individual inclusions

\section{A.23.1 Obl-I1}

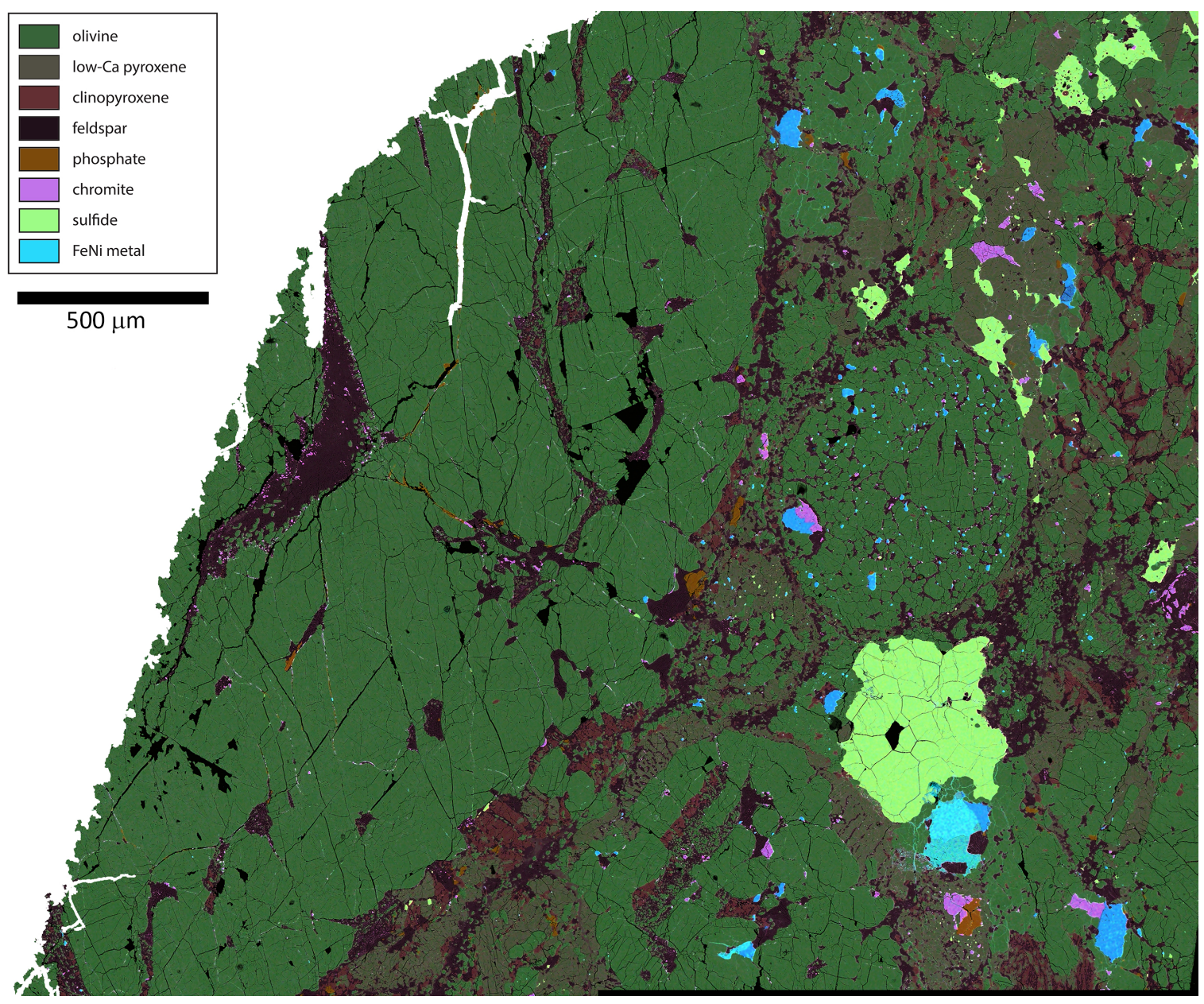

Figure A.56: False-color EDS phase map of Obl-I1.

Only a small fragment of this inclusion is available on this thin section, but based on its curvilinear contact with the host it may have originally had an apparent diameter of over $6 \mathrm{~mm}$. It is in sharp but jagged contact with the host, and appears to be a broken fragment of a larger object.

Mineralogically, it is almost entirely composed of coarse-grained olivine, the crys- 
A Chemical and petrographic descriptions of individual inclusions

tals of which are over $1 \mathrm{~mm}$ in size. A glassy, feldspathic mesostasis is interstitial to the olivine. Minute $(<10 \mu \mathrm{m})$ chromite blebs are common within the mesostasis, as are small $(10-20 \mu \mathrm{m})$, anhedral calcic pyroxene and low-Ca pyroxene grains. Very few grains of phosphate, FeNi metal, and sulfide occur with the mesostasis.

The olivine and low-Ca pyroxene are both equilibrated, with average compositions Fa $28.8 \pm 0.6(\mathrm{~N}=14)$ and Wo $1.2 \pm 0.1$, Fs $23.6 \pm 0.4$, and En $75.3 \pm 0.4(\mathrm{~N}=$ 5), respectively. The diopside is also equilibrated, with average composition Wo 45.5 \pm 0.3 , Fs $8.2 \pm 0.2$, and En $46.3 \pm 0.2(\mathrm{~N}=14)$. The feldspar is more variable in composition, averaging $\mathrm{Ab} 82.3 \pm 2.1$, Or $4.0 \pm 1.1$, and An $13.7 \pm 1.9(\mathrm{~N}=$ 14). Olivine, low-Ca pyroxene, diopside, feldspar, and chromite compositions were determined with EDS point spectra. Phosphate and metal were characterized with EDS map extracts and AZtec's analyze phases. 
A Chemical and petrographic descriptions of individual inclusions

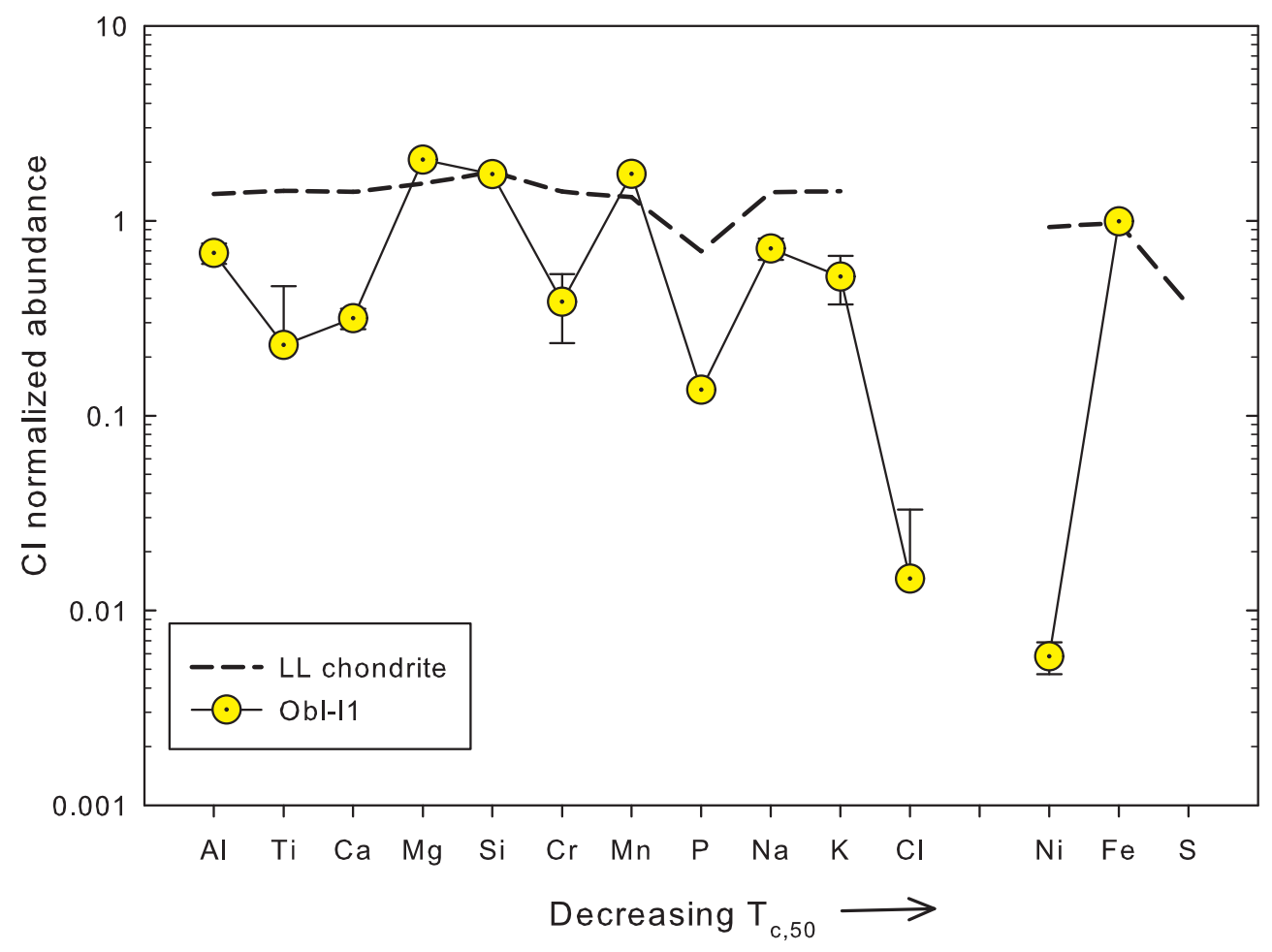

Figure A.57: CI-normalized bulk chemistry of Obl-I1. This inclusion is almost entirely olivine, and as it is only a small fragment is likely unrepresentative. 